\author{
Université Libre de Bruxelles \\ Faculté des Sciences Appliquées \\ Service de Mathématiques de la Gestion
}

\title{
A MULTICRITERIA PERSPECTIVE ON REVERSE AUCTIONS
}

\author{
Yves De Smet \\ Promoteur: Professeur Philippe Vincke
}

Thèse présentée en vue de l'obtention du grade académique de Docteur en Sciences Appliquées

Année académique 2005-2006 

An expert is a man who has made all the mistakes, which can be made, in a very narrow field.

Niels Henrik David Bohr

The purpose of models is not to fit the data but to sharpen the questions.

Samuel Karlin 


\section{Acknowledgements}

La réalisation de cette thèse de doctorat a été, avant tout, une expérience humaine. Un cheminement pavé d'incertitudes, de questionnements, de réflexions, ... une aventure au cours de laquelle j'ai appris énormément de choses, dont beaucoup dépassent le cadre scientifique. En tant que telle, elle ne saurait être réduite au fruit d'un travail isolé. Au contraire, de nombreuses personnes ont contribué, directement ou indirectement, à son aboutissement. Je voudrais, ici, les remercier.

Tout d'abord, je remercie le Professeur Philippe Vincke qui a accepté de diriger cette recherche. En tant que directeur de thèse, je lui suis reconnaissant de m'avoir laissé de grandes libertés, tant concernant le sujet à traiter que la manière de l'aborder. En n'étant pas directif, il a su donner à cette thèse une très forte connotation personnelle. A présent, cet aspect reste l'un des plus importants et des plus riches à mes yeux. Ses nombreux conseils et critiques ont, par ailleurs, grandement facilité mon travail. Au-delà du cadre de la thèse de doctorat, je lui suis également très reconnaissant de m'avoir offert de nombreuses opportunités d'épanouissement au cours de ces cinq dernières années.

Je tiens à remercier Messieurs les Professeurs Hugues Bersini, Bertrand Mareschal, Marc Pirlot, Daniel Vanderpooten et Philippe Van Ham pour avoir accepté de faire partie du jury chargé d'évaluer ce travail. De plus, je remercie les Professeurs Hugues Bersini, Bertrand Mareschal et Philippe Van Ham pour avoir fait partie du comité d'accompagnement de ma thèse de doctorat.

La recherche est une activité qui ne s'improvise pas. Elle s'apprend. Pour ma part, cet apprentissage a été favorisé par Messieurs les Professeurs Jean-Pierre Brans, Pierre-Louis Kunsch, Bertand Mareschal, José Figueira et Johan Springael qui n'ont jamais hésité à m'associer à leurs travaux. Je les en remercie. 
$\mathrm{Au}$ cours de la rédaction de cette thèse de doctorat, j'ai pu bénéficier des remarques et critiques pertinentes de nombreuses personnes. En particulier, j'aimerais chaleureusement remercier Pierre-Louis Kunsch, Claude Lamboray, Bertrand Mareschal, Linett Montano Guzmán, Philippe Nemery de Bellevaux et Marc Pirlot.

De façon plus générale, en plus des personnes précitées, je remercie toutes celles avec lesquelles j'ai eu l'occasion de discuter de sujets en lien direct ou non avec mon thème de recherche. En particulier, les membres de l'équipe du Service de Mathématiques de la Gestion, Flavien Balbo, Kim Cao-Van, Michael Daubie, Patrick Meyer, Céline Mousset, Stefan Strecker, ...

Je tiens à remercier Madame Pivin, Professeur de Mathématiques à l'Athénée Fernand Blum, pour m'avoir encouragé à entreprendre des études en Sciences Mathématiques.

En dehors du cadre scientifique, je voudrais remercier mon ami Olivier Colin, pour m'avoir soutenu au cours des moments difficiles qui sont survenus pendant la rédaction de cette thèse. Merci aussi à Nicolas Boulanger et à Christophe Bruylandts.

Pour conclure, toute ma gratitude va à mes parents qui m'ont appris tant de ces choses qu'on ne trouve pas dans les livres, notamment avoir des idéaux et les réaliser. Je remercie également ma marraine qui m'a toujours soutenu dans ce que j'entreprenais.

Enfin, je voudrais remercier Mademoiselle Fatoumata Camara, qui a été présente à un moment où j'avais besoin d'elle et qui m'a redonné goût à ces choses simples de la vie, en particulier, à la recherche. 


\section{Contents}

Acknowledgements

Table of contents vii

List of figures $\quad x$

List of tables $\quad$ xii

Conventions $\quad$ xiii

$\begin{array}{lr}\text { Introduction } & 1\end{array}$

$\begin{array}{llr}\text { I } & \text { Preliminary notions } & 6\end{array}$

1 A brief introduction to Auctions $\quad 7$

1.1 Introduction . . . . . . . . . . . . . . . 7

1.2 Auctions' basics . . . . . . . . . . . . . . . . . 10

1.3 The Independent Private Values model . . . . . . . . . . . . . 13

1.4 A few selected extensions . . . . . . . . . . . . . . . . 16

1.5 e-Auctions . . . . . . . . . . . . . . . . . . . 19

1.5.1 The case of eBay . . . . . . . . . . . . . . . 21 
1.6 A global perspective . . . . . . . . . . . . . . . . . 22

1.7 Conclusion . . . . . . . . . . . . . . . . . . . . . 22

2 Multicriteria Decision Aid 24

2.1 Introduction and motivations . . . . . . . . . . . . 24

2.1.1 Decision aid ................. 25

2.1.2 The multicriteria paradigm . . . . . . . . . . 26

2.2 Basic terminology and problem's formulation . . . . . . . . 27

2.2.1 The set of actions and their consequences . . . . . . 27

2.2.2 Preference modelling . . . . . . . . . . . . 30

2.2.3 Consistent family of criteria . . . . . . . . . . 33

2.2.4 The multicriteria decision aid problems . . . . . . . . 34

2.3 Methods ..................... . . 35

2.3.1 Multiple attribute utility theory . . . . . . . 36

2.3.2 Outranking methods . . . . . . . . . . . . . . . 40

2.3.3 A few selected modeling issues . . . . . . . . . . . 48

2.4 Conclusion . . . . . . . . . . . . . . . . . 50

3 Multi-attribute auctions $\quad 52$

3.1 Introduction $\ldots \ldots \ldots \ldots \ldots \ldots \ldots \ldots \ldots$

3.2 A few first observations . . . . . . . . . . . . . 55

3.3 Playing with several attributes: different approaches . . . . . . 57

3.4 A common framework . . . . . . . . . . . . . . 60

3.5 How to characterize the mechanisms ? . . . . . . . . 62

3.6 A few experimental results . . . . . . . . . . . . . . 64

3.7 A few selected additional research questions . . . . . . . . 69

3.8 Conclusion . . . . . . . . . . . . . . . . . . . . 72 
II New developments

4 Multicriteria auctions: a theoretical framework

4.1 Introduction . . . . . . . . . . . . . . . . 74

4.2 The management of incomparability between multidimensional

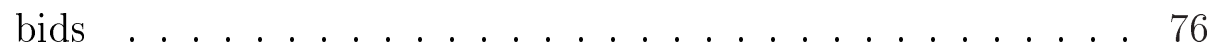

4.3 Problem's formalization . . . . . . . . . . . . . 77

4.3.1 Main concepts and definitions . . . . . . . . . . . 78

4.3 .2 Bids comparison $\ldots \ldots \ldots \ldots$

4.3 .3 Multicriteria auction . . . . . . . . . . . . . 80

4.3 .4 Discussion . . . . . . . . . . . . . . . . . . . . . . 82

4.4 Properties . . . . . . . . . . . . . . . . . . . . . . . . 89

4.4.1 Properties related to the winning set . . . . . . . 90

4.4.2 Characterization of the winning bids . . . . . . . . 90

4.5 An illustrative example based on the Electre I method . . . . 98

4.6 Extensions to other auction formats . . . . . . . . . . . . 103

4.6.1 Vickrey multicriteria auctions . . . . . . . . . . . 103

4.6.2 Dutch multicriteria auctions . . . . . . . . . . . . 105

4.7 Particularizations to existing approaches . . . . . . . . 109

4.7.1 Uni-criterion auctions . . . . . . . . . . . . . . . . . 109

4.7.2 Dominance based multicriteria auctions . . . . . . . . 110

4.7.3 Multi-attribute auctions . . . . . . . . . . . . . . 112

4.8 Related questions . . . . . . . . . . . . . . . . . . 115

4.8.1 Multicriteria auctions versus RFQ . . . . . . . . . 115

4.8.2 Ex-post versus integrated bids selection . . . . . . . 120

4.9 Conclusion . . . . . . . . . . . . . . . . . . . . 125 


\section{Butterfly auctions}

5.1 Introduction and motivations f . . . . . . . . . . . 127

5.2 The model . . . . . . . . . . . . . . . . . . . . . . . . . 128

5.2.1 Differences and preference degrees per criterion . . . 129

5.2.2 Preference degrees aggregation . . . . . . . . . . 130

5.2.3 The outranking relation $\mathcal{S} \ldots \ldots$. . . . . . . . 131

5.2 .4 Discussion . . . . . . . . . . . . . . . . 135

5.3 Properties of the butterfly model . . . . . . . . . . . . . . 139

5.4 Related questions . . . . . . . . . . . . . . . . . . . 141

5.4.1 Bids submitted and preference elicitation . . . . . . . . 142

5.4.2 Bid decrement and indifference thresholds . . . . . . . 144

5.4.3 Multicriteria bidding support tool . . . . . . . . . . 147

5.4.4 Some reflections about robustness . . . . . . . . . . 149

5.5 An illustrative example . . . . . . . . . . . . . . . . . . 151

5.6 Conclusion . . . . . . . . . . . . . . . . . . . . . . 154

6 Bidding Niches 156

6.1 Introduction . . . . . . . . . . . . . . . . 156

6.2 Formalization . . . . . . . . . . . . . . . 161

6.2.1 Improvements'quantification . . . . . . . . . . . . . 161

6.2.2 The notion of competition and related issues . . . . . 162

6.2 .3 IS partitions . . . . . . . . . . . . . . . . 163

6.2.4 Bidding niches partitions . . . . . . . . . . . . . 164

6.3 Hierarchical algorithm . . . . . . . . . . . . . . . 166

6.4 Empirical tests . . . . . . . . . . . . . . . . 167

6.4.1 A few words about the generation of artificial data sets 169 
6.4.2 Estimation of the number of cone partitions . . . . . 171

6.4.3 Testing the hierarchical algorithm on artificial data sets 173

6.4.4 Testing the assignment'sensitivity with respect to the $\delta$ parameters . . . . . . . . . . . . 173

6.4.5 Testing the assignment's sensitivity with respect to the improvement of offers . . . . . . . . . . 176

6.4.6 Simultaneous versus distinct management of bidding niches . . . . . . . . . . . . . . . . . . 179

6.5 Conclusion and directions for future research . . . . . . . . . 181

Conclusion

7 Rand index testing

Appendix

Bibliography 


\section{List of Figures}

1.1 The selling of UMTS licenses: the example of 5 neighbouring states. . . . . . . . . . . . . . 17

2.1 Generalized criterion of type $5 \ldots \ldots 44$

2.2 Promethee I partial ranking . . . . . . . . . . . . . . . . . 47

2.3 Promethee I partial ranking, when bid $b_{3}$ is not considered anymore. . . . . . . . . . . . . . . 48

3.1 The Leap Frog method: example of a potential outcome . . . 58

3.2 Dominance based auction: example of a potential outcome . . 59

4.1 Example of a 2-attributes context with two bidding niches. . . 78

4.2 Example of an auction context where the mixed transitivity assumption is not satisfied . . . . . . . . . . . . 81

4.3 Illustration of a 2 criteria auction context with 2 distinct winning sets. $W S_{1}=\left\{b_{1}=(4,4)\right\}, W S_{2}=\left\{\tilde{b}_{1}=(3,5)\right\}$. How-

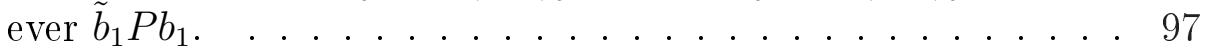

4.4 Illustration of a Vickrey multicriteria auction based on the dominance relation. . . . . . . . . . . . . 105

4.5 Dominance based multicriteria auctions. . . . . . . . . . . 111

4.6 Illustration of a multi-attribute auctions. . . . . . . . . . . . 114

4.7 Multi-attribute auctions: different set of weights can lead to select the same winning bid. . . . . . . . . . . . 116 
4.8 Mean percentage of preferences revealed when the number of sellers varies from 2 to $100 \ldots \ldots \ldots$. . . . . . . 118

4.9 Mean utilities for first and second preferred bids (continuous lines). The dots represents mean utilities under the moderate bidding behavior. . . . . . . . . . . . . . . . . . . 119

4.10 Evolution of the utility gap rate: difference between the moderate bidding score and the first score divided by the difference between the second and the first score when the number of sellers varies from 2 to 100 . . . . . . . . . . . . 120

4.11 Preference revelation scheme . . . . . . . . . . . . . . . 121

4.12 Integrated versus ex post bids selection in a 2-attributes auction. 122

4.13 Ex-post versus integrated bids selection. Evolution of the mean (winning) utility in these two scenarios when the number of bidders range from 2 to 50 and the number of attributes varies from 2 to $5 \ldots \ldots \ldots$. . . . . . . . . . 123

4.14 Ex-post versus integrated bid selection. The ratio $U_{(1)}^{\text {expost }} / U_{(2)}$ remains stable if we consider all the bids or only the bids lying in the dashed square. . . . . . . . . . . . . . . . . 124

5.1 Linear preference function transforming differences between two bids according to a specific criterion into preference degrees for this criterion. . . . . . . . . . . . . . 130

5.2 Illustration of a 2-dimensional butterfly model. . . . . . . . . . 133

5.3 Potential evolution of a 2-dimensional Butterfly: the comparability window is progressively extended while the set of admissible weights is refined. . . . . . . . . . . . 134

5.4 Evolution of the mean percentage of good "classifications" when the size of the training set evolves from 2 to 30 . The different curves correspond to values of d ranging from 2 to 5 . 144

5.5 Evolution of the mean percentage of points that were classified as preferred by both models $(\beta$ and $\tilde{\beta})$ when the size of the training set evolves from 2 to 30 . The different curves correspond to values of $\mathrm{d}$ ranging from 2 to $5 . \ldots 145$ 
5.6 Illustration of a decrement step $\epsilon$ in a multi-attribute utility auction. . . . . . . . . . . . . . . . 146

5.7 Illustration of indifference thresholds in a particular butterfly model. . . . . . . . . . . . . . . . . . . . . . 147

6.1 The transport problem: illustration of 2 bidding niches (delimited by the dashed lines). . . . . . . . . . . . . . 157

6.2 The advertisement problem: illustration of 3 bidding niches. . 158

6.3 The procurement of a company's cleaning contract: illustration of outlying bids. . . . . . . . . . . . . . . . . 158

6.4 The transport problem (bis). . . . . . . . . . . . . 160

6.5 Illustration of a unique bidding niche. . . . . . . . . . . . . 160

6.6 Additivity of the $\iota$ function . . . . . . . . . . . . . 162

6.7 The competition of an offer $b$ with respect to a subset of offers

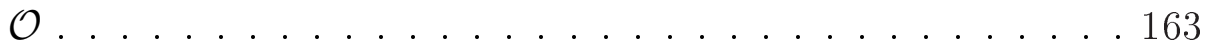

6.8 Example of a data set with 2 niches of 5 observations. . . . . 170

6.9 Example of offers'improvement. Initial offers are represented by dots. . . . . . . . . . . . . . . . . . . . . . . 179 


\section{List of Tables}

1.1 Statistics and recent evolution of eBay - Second Quarter 2004 and $2005(41) \ldots \ldots \ldots \ldots \ldots \ldots$

1.2 Classification of auction situations based on their characteristics 23

2.1 Example of condorcet effect. . . . . . . . . . . . . . . 32

2.2 Example of 4 bids leading to a preferential dependence situation. 37

2.3 Bidding support system. Different bid configurations. Utility

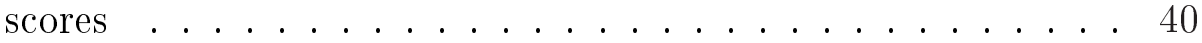

2.4 Bidding support system. Different bid configurations. Margin for supplier $s_{i} \ldots \ldots \ldots \ldots \ldots \ldots \ldots$

3.1 Multidimensional auctions . . . . . . . . . . . . . 70

4.1 Seller's best contract evaluated according to the 3 criteria . . . 99

4.2 Mobile phone contract procurement auction. Total cost, for each seller, evaluated on three particular scenarios. . . . . . . 99

4.3 Mobile phone contract procurement auction. Application of the Electre I. Illustration of one potential auction realization. 100

4.4 Mobile phone contract procurement auction. Application of the Electre I. Illustration of a second auction realization. . . . 101

4.5 Electre I based multicriteria auction- continued . . . . . . . . 102

4.6 Mobile phone contract procurement auction. Illustration of Dutch multicriteria auctions based on the Electre I. realization. 107 
5.1 Seller's best contract evaluated according to the 2 criteria . . . 152

5.2 Mobile phone contract procurement auction. Application of the butterfly model. Illustration of one potential auction realization. . . . . . . . . . . . . . . . . . 152

5.3 Mobile phone contract procurement auction. Illustration of algorithm 4. . . . . . . . . . . . . . . . . 154

6.1 Structure of the tests performed. . . . . . . . . . . 168

6.2 Minimal distance between two niches and percentage of overlapping niches for data sets generated with $\alpha=0.75 \ldots 171$

6.3 Average number of cone partitions for different combinations of the number of observations and criteria. . . . . . . . . 172

6.4 Parameters of model $N \bar{C} P=a^{n} \cdot b \ldots \ldots$. . . . . . . . . 172

6.5 Average $R$ and percentage of cases with a lower or equal quality than the expected quality for data sets with $\alpha=1 \ldots 174$

6.6 Average $R$ and percentage of cases with a lower or equal quality than the expected quality for data sets with $\alpha=0.75 \ldots 175$

$6.7 R$ average for 2 niches. . . . . . . . . . . . . . . . . 175

6.8 Sensitivity analysis for data sets with $\alpha=1$ and 5 observations 177

6.9 Dynamic evolution $(p=0.2)$ for data sets with $\alpha=1$ and 5 observations . . . . . . . . . . . . . 178

6.10 Dynamic evolution $(p=0.4)$ for data sets with $\alpha=1$ and 5 observations . . . . . . . . . . . . . 180

6.11 Competition decrease -5 observations per niche . . . . . . 181

6.12 Competition decrease -7 observations per niche $\ldots . . . .181$

7.1 Estimated $95 \%$ percentile Rand index between two random partitions ....................... 188 


\section{Conventions}

The following conventions are used through the present work:

- the $\neg$ symbol is used to denote the negation.

- $q$ denotes the number of criteria.

- $e_{j}$ denotes a vector of $q$ components such that the only non null component is the $j^{\text {th }}$ one and is equal to 1 .

- $b$ denotes a bid, i.e., in the context of multicriteria auctions, a vector of $q$ components. These components are referred to as attributes.

- $b^{j}$ is referred to as the $j^{\text {th }}$ attribute of the bid $b$. If the component's index is omitted all the attributes are considered. By extension, $b^{-j}$ denotes all the attributes except the $j^{\text {th }}$ one.

- we assume that all the criteria have to be minimized. We will explicitly warn the reader when a specific criterion has to be maximized.

- Let $\prec$ denotes the Pareto dominance relation i.e. $b \prec \tilde{b} \Leftrightarrow b^{j} \leq \tilde{b}^{j} \forall j \in$ $\{1, \ldots, q\}$ and $\exists \tilde{j} \in\{1, \ldots, q\} \mid b^{\tilde{j}}<\tilde{b}^{\tilde{j}}$. Additionally, we define $\preceq$ as follows: $b \preceq \tilde{b} \Leftrightarrow b^{j} \leq \tilde{b}^{j} \forall j \in\{1, \ldots, q\}$. (The symbol $\succ$ and $\succeq$ are defined in a similar way).

- Multicriteria auctions are viewed as successions of offers. Consequently, $b(k)$ denotes the $k^{t h}$ bid received by the auctioneer and $t_{k}$ denotes its related time (we implicitly assume that the auction starts at time 0 . So, for instance, $t_{3}=10$ seconds expresses the fact that the third bid has been received 10 seconds after the auction beginning). By abuse of language, we will often refer the index $k$ to as the time the bid was received (i.e $t_{k}$ ). Finally, let us stress that the index $k$ is only specified when the underlying information is needed. 
- $b_{i}$ denotes a bid proposed by the $i^{\text {th }}$ bidder. This index is also specified only when the underlying information is needed.

- In brief:

- $b$ denotes any bid received in the auction.

- $b_{i}$ denotes a bid proposed by the $i^{\text {th }}$ bidder.

- $b^{j}$ denotes the value of the $j^{\text {th }}$ attribute of the bid $b$.

- $b(k)$ denotes the $k^{\text {th }}$ bid received during the auction (proposed by any bidder).

- $b_{i}^{j}$ denotes the value of the $j^{t h}$ attribute of a bid $b$ proposed by the $i^{\text {th }}$ bidder.

- $b_{i}(k)$ denotes the $k^{t h}$ bid proposed by the $i^{\text {th }}$ bidder.

- $b^{j}(k)$ denotes the value of the $j^{\text {th }}$ attribute of the $k^{\text {th }}$ bid received during the auction (proposed by any bidder).

- $b_{i}^{j}(k)$ denotes the value of the $j^{\text {th }}$ attribute of the $k^{\text {th }}$ bid proposed by the $i^{\text {th }}$ bidder. 


\section{Introduction}

\section{Context and objectives}

Nowadays, electronic commerce has almost entered everybody's life. At first, one may think about business to consumer (B2C) applications. A few years ago, particular web sites like Amazon.com were cited to illustrate this trend. Today, common brands, local supermarkets, travel agencies, financial institutions, ... have their own electronic commerce portal and every kind of products or services are sold in this way, even highly specific ones ${ }^{1}$.

Obviously, electronic commerce cannot be reduced to conduct traditional commercial activities through new electronic channels. If the technological aspect is important, other factors play a central role in its successful development. Among them, the market design remains crucial. Trading on the Internet is, in many aspects, different than trading in a classic way. Therefore, some common practices have to be adapted while new ones have appeared.

In this context, auctions have received great attention: they have not only been used for $\mathrm{B} 2 \mathrm{C}$ or consumer-to-consumer $(\mathrm{C} 2 \mathrm{C}$ ) applications (with success stories such as eBay) but also to model negotiation between computerized self-interested agents. On the other hand, the emergence of e-Business has favored extensions of classic auction mechanisms to, for instance, multi-unit and multi-object auctions.

Among the business-to-business (B2B) applications, e-Procurement remains an active field of research. One way to tackle this problem relies on the use of reverse auctions (i.e. one buyer and several sellers). For instance,

\footnotetext{
${ }^{1}$ The famous motorcycles constructor Ducati decided to sell a limited edition (2.000 units) of the MH900e using exclusively the Internet. The sell started on January $1^{\text {st }} 2000$, at 00:01 a.m. and finished 31 minutes later (58)
} 
in 2000, General Motor, Ford and Daimler-Chrysler joined together to create an electronic market place based on auctions, called Covisint, for the procurement of basic elements (common plastic parts, bolts, steal,...). Renault and Nissan joined the group some time later (nowadays Covisint has been integrated in Ariba (5)) . Another example is the WorldWide Retail Exchange (126) which is an electronic market place based, among others, on e-auctions for the sourcing in the retail and consumer goods industry.

However, as stressed by P.Milgrom (79) ... price-only auctions are of little value if the goods, or their suppliers, have widely varying characteristics, because the buyer will not normally buy based on a comparison of prices alone. In the automobile example mentioned above, price-only auctions are not suited for the procurement of elements requiring detailed technical specifications or an important implication of the supplier in the design.

As a consequence, some researchers started to consider auction mechanisms that support bids characterized by several attributes in addition to the price (quality of the product, quantity, terms of delivery, quality of the supplier, ...). These are referred to as multidimensional, multi-attribute, multiple issue or scoring auctions ${ }^{2}$. In this $\mathrm{PhD}$ thesis, we propose a new contribution to this emerging field.

A typical example of such a situation is the procurement of computer units. A company has to buy a fixed number of desktop computers. Therefore, it specifies the minimal requirements and invites bidders to propose offers not only for the price but also on other negotiable attributes (hard disk or memory capacity, CPU, number of units, warranty terms, ... ).

Methods involving the simultaneous optimization of several criteria are not new: they have been developed and tested for about 40 years. Among them, those proposed within the so-called French school of Multiple Criteria Decision Aid (MCDA) have the particularity not to impose complete relations for the comparison of alternatives (i.e. not to force the comparison between any pair of alternatives). By doing so (i.e. by allowing potential incomparability between some pairs of alternatives), the preferences of the decision maker are represented in a more flexible way and distinctive features of the problem (such as contradictory elements) may be outlined.

In this spirit, the main objective of this $\mathrm{PhD}$ thesis is to investigate the use of partial relations in multi-attribute auctions. In order to avoid ambiguity

\footnotetext{
${ }^{2}$ in what follows we will use the word multi-attribute auctions to refer to this specific field
} 
with the latter term, this will be referred to as multicriteria auctions. If the use of partial relations is still subject to criticism in the multiple criteria decision making community, it is even harder to justify it in a competitive context such as auctions where, precisely, all the bids are (have to be ?) comparable. Through this work, our aim will be to highlight the potential interest of such an approach. This will lead us both to consider the problem from an abstract point of view and to develop a specific model. Finally, the context of bidding niches will be presented and formalized in order to motivate the use of multicriteria auctions.

\section{Thesis structure}

Basically, this document is organized in two main parts. The first one, including chapters 1,2 and 3, is dedicated to the preliminary notions needed to understand the new developments presented in the second part, i.e. in chapters 4,5 and 6 .

As already stressed, this $\mathrm{PhD}$ thesis is at the cornerstone of two main fields: multicriteria decision aid and auctions. Chapter $\mathbf{1}$ constitutes a brief introduction to the latter. The emphasis will be put on the basic terminology, on the description of some main auction mechanisms and on recent developments such as combinatorial auctions or electronic auctions. The aim of this chapter is not only to introduce auctions but also to make the reader sensitive to the richness of this research field. It also shows that, if auction theory was at first essentially studied by means of statistical and probabilistic tools, it relies nowadays more and more on operational research tools. Therefore, a link with decision aid tools seems to follow a natural trend.

Chapter 2, dedicated to multicriteria decision aid, further completes the introduction initiated in the first chapter. The multicriteria tools, used in subsequent chapters, will be presented and criticized. More precisely, our attention will be focused on the approaches of the so-called French school. If several authors have already considered extensions of auctions incorporating traditional multicriteria tools (such as additive linear multi-attribute utility functions), this work is, to our knowledge, the first one to propose extensions characterized by partial preference relations.

Once the two background fields have been outlined, we present in chapter 3 some existing contributions related to multi-attribute auctions. Since the beginning of the $21^{\text {th }}$ century, due to the emergence of electronic market 
places, this field has known an impressive evolution. Economists, mathematicians, computer scientists, engineers, ..., have contributed to its development: each of them looking at the problem from a specific perspective. This chapter illustrates a variety of research questions that have already been addressed.

The second part of this work is dedicated to new developments. In chapter 4, we have decided to investigate the field at an abstract level i.e. considering a general preference structure $(P, I, J)$. This has led us to define what we call a multicriteria auction, to formalize related notions and to study their properties. In these sections, a central concern is to study the auction outcomes. Then extensions to Vickrey and Dutch multicriteria auctions are sketched and particularizations to existing approaches such as uni-criterion, dominance based or multi-attribute auctions are presented. The chapter ends with considerations both about the comparison between multicriteria auctions and request for quotes and about ex-post versus integrated bid selection. Of course, the fact that two bids could, potentially, be incomparable is motivated and commented through the chapter.

In chapter 5, we introduce a particular model, referred to as the Butterfly model, to apply multicriteria auctions. Inspired both by the Electre and Promethee approaches, it is distinguishing itself from traditional methods in the sense that it can be dynamically adapted during the auction. Starting with a very general version of the model (that could be limited to the dominance relation), the buyer is allowed to progressively refine it. These updates are the consequence of bids comparisons or direct constraints imposed by the decision maker. Questions related to preference elicitation, the role of indifference parameters and bidding support tools are discussed. The formalization of the model, its properties and its distinctive features are then presented on an illustrative example.

When the bidding space is not homogeneous, the use of partial relations allows the buyer to manage different bidding niches within the same auction. Consequently, the auction may end with distinct winning offers (that together are mutually incomparable) each of them characterizing one typical niche. Chapter 6 is devoted to the formalization of a novel concept: the partition of the bidding space into bidding niches. First of all, a few examples are outlined in order to illustrate this idea. Then a model to detect bidding niches partitions and a related hierarchical algorithm are presented. Finally, several empirical tests are conducted, among others, to validate the algorithm, to evaluate its sensitivity to parameters and to study the dynamic aspect of the problem. 
Many questions are addressed through this thesis. If some answers have been proposed, some of them remain open while new ones have appeared. The conclusion of this work will summarize our contribution and list the numerous directions for future research.

To conclude, let us stress that a number of personal contributions have directly, or indirectly, inspired the redaction of this work. First of all, many of the ideas and developments presented in the second part of this document can be found in the paper Multicriteria auctions without full comparability of the bids (39). Additionally, some of the introductory ideas presented in chapter 4 can also be found in Multicriteria Auctions: an Introduction (36). The butterfly model presented in chapter 5 is also discussed in Butterfly auctions: clustering the bidding space (37). Finally different works about multicriteria clustering methods $(33 ; 38 ; 82 ; 46)$ are at the root of chapter 6 . The proposed model and the related empirical tests are partially summarized in Clustering the bidding space into niches: a multicriteria hierarchical approach (40). 


\section{Part I}

\section{Preliminary notions}




\section{Chapter 1}

\section{A brief introduction to Auctions}

\subsection{Introduction}

Auctions are used everyday to buy (or sell) objects, artefacts, services, commodities, financial products, ... Moreover, their recent application to ecommerce, with success stories such as eBay (41), has popularized their use by anybody: from the 7 years old schoolboy to the 77 years old retired man!

The first testimonies of the use of auctions date back from the fourth century BC: the Greek historian Herodotus described the sale of future wives in Babylon (87) using an auction mechanism. Later, in $197 \mathrm{AC}$, the whole Roman Empire was sold using an auction mechanism (67). At that time, the new emperor had to offer an accession premium to the Praetorian guards to buy their protection and, consequently, justify his new position. After the murder of Pertinax, two candidates came to the Praetorian guards to buy their services. In order to decide between the two, the soldiers organized an auction.

Usually, the general public associates, nearly exclusively, the use of auctions to auction houses like Christie's (28) and Sotheby's (107). This view is, however, a bit reductive. The following examples illustrate the wide variety of auctions' applications. 
- Rare items, paintings, sculptures, ... (52)

- The works of Pablo Picasso have been sold at auction no fewer than 3.579 times for a total value of 1.094.386.813\$ (data from May 1997).

- A volume of nine complete symphonies in Mozart's handwritting has been sold at Sotheby's on May 221987 for a record amount of $4.100 .000 \$$.

- The sculpture "Grande Femme Debout" of Alberto Giacometti has been sold at Christie's on November 82000 for 14.306.000\$.

$-\ldots$

- Fish, livestock $(113 ; 108)$, tobacco $(108), \ldots$

Auctions are used everyday in small, medium and large fish centers (108). For instance, Noriega (84) describes in details the local fish market in Blanes (Catalonia, Spain). Inspired by this institution, he has developed a platform to support multi-agent interactions.

\section{- Flowers}

In the Netherlands, flower markets represent an important economic activity. For instance, in Aalsmeer (15) ${ }^{1}$, one sells, everyday, on average more than 20.000.000 flower sets and the daily turnover is about 6.000.000 Euros. This activity is based on a particular auction mechanism: the Dutch auction (see section 1.2).

\section{- Wine}

The Chicago Wine Company (117) organizes several auctions per month to sell bottles of wine. For instance, between January 1996 and February 2004, 1.246.975 bottles of red bordeaux have been sold for an average price of $117.15 \$$. During that period, the highest price $(8.200 \$)$ has been reached for a bottle of Margaux dating back to the year 1900 .

\section{- Collectibles}

With the emergence of web sites like eBay, the cost to organize auctions has been impressively lowered. Therefore, lower value items such as advertising items, comics or baseball cards have been sold using auctions. If, initially, the famous website eBay (41) was designed to trade collectible Pez dispensers, it hosts, nowadays, around fifty main categories just for collectibles (29) (each of them being divided in thousands of subcategories - see section 1.5.1 for more statistics about eBay).

\footnotetext{
${ }^{1}$ with about $1.000 .000 \mathrm{~m}^{2}$ of floor space, this auction house is the largest commercial building in the world.
} 


\section{- Financial products}

In the United States, treasury bonds are regularly sold using sealed bid auctions: three and six months T-bills are auctioned every Monday (the total volume of Treasury securities auctioned in 1995 was about 2 trillion dollars) (89). Moreover, financial markets, such as for instance the New York Stock Exchange (NYSE), are based on a particular auction mechanism: the double auction (see section 1.4).

\section{- Electricity (73)}

With the liberalization of the electricity markets, electricity trading has became a key issue of the sector. The design of markets such as the Amsterdam Power Exchange (APX) (4) or the European Energy Exchange (EEX) (44) heavily rely on auctions.

\section{- Telecommunication and Spectrum Auctions (76)}

In 1993, the Federal Communication Commission (FCC (45), USA) used particular auction mechanisms for the sale of more than $\$ 20$ billion of spectrum licenses (73). Since the first spectrum right auctions conducted by the New Zealand government in 1990, the related research has been very active, especially with the development of combinatorial auctions (see section 1.4 for details).

\section{- Privatization}

Auctions have been used by several governments for the privatization of nationally owned companies (among others in countries from the former Soviet bloc (51)). For instance, Yukos (131), which is, nowadays, a leading petroleum company, has been sold in the mid 1990s by the Russian government to private investors through a series of tenders and auctions.

\section{- Procurement auctions}

Procurement is a key activity for governments, industries, companies, ... With the development of electronic commerce, electronic procurement and, more specifically, electronic reverse auctions are more and more common. For instance, Kodak has recently decided to use electronic reverse auctions for at least $20 \%$ of its annual purchase (which are around $\$ 6$ billion) (91). Procurement auctions will be further discussed in chapter 3

Since the works of William Vickrey (124), the literature about auctions has known a significant expansion. As pointed out by Krishna (67), the Econ Lit database has more than a thousand entries with the word "auction 
" or "auctions". The impressive review done by Paul Klemperer (64) further testifies this activity.

As a consequence, the only ambition of this chapter is to, superficially, introduce the field and to define the basic vocabulary that will be used in the subsequent chapters.

This chapter is organized as follows. In section 1.2, the basic terminology of auction theory will be introduced and the four traditional auction mechanisms will be described. Then, in section 1.3, a classic theoretical model, the independent private value model, will be briefly outlined. A few recent extensions of traditional auction theory will be presented in section 1.4 and section 1.5 will be devoted to electronic auctions. Finally, a global classification of auction mechanisms will be presented in section 1.6.

To conclude, let us stress that the present chapter will be further completed by chapter 3 which is devoted to multi-attribute auctions.

\subsection{Auctions' basics}

In this section, we will always consider the following context: several buyers are competing to buy an unique item from a seller. This is referred to a forward auction, or simply an auction. The term item will be used to refer to what's being sold: the object, the service, the contract, ... A bidder is a buyer who submits an offer or a bid (84). The auctioneer is the agent who, in representation of a seller, conducts an auction.

Basically, an auction is a market institution with an explicit set of rules determining allocation and prices on the basis of bids from market participants (75). In traditional auction theory, one usually distinguishes four basic kinds of auctions:

\section{- English auctions}

The auctioneer starts the auction by calling out a low price. Then, bidders propose offers the price of which is higher than the current best bid. The auction stops as soon as no more bidder outbid the current best offer during a predefined delay. The one who proposed the last best bid wins the item and pays a price equal to his bid. 


\section{- Dutch auctions}

The auctioneer starts the auction by calling out a sufficiently high price $^{2}$. Then, he continuously decreases the price until one bidder reacts and decides to buy the item at that price.

\section{- First Price Sealed Bid auctions}

Each bidder proposes a sealed offer to the auctioneer. Once he has received all the offers, the auctioneer ranks them from the best to the worst. The bidder who proposed the highest bid wins the item and pays a price equal to his bid.

- Second Price Sealed Bid auctions (also called Vickrey auction) Second Price Sealed Bid auctions are close to First Price Sealed Bid auctions. It is still the bidder who proposed the highest offer who wins the item but he only has to pay a price equal to the second highest bid.

Analyzing these different auction mechanisms we can make a distinction regarding the nature of the bids. In sealed bid auctions, the offers proposed to the auctioneer are "secret" (since, as it is pedagogically explained in the auction literature, they are communicated in sealed envelopes). On the contrary, in open auctions, i.e. English and Dutch auctions, the offers are public 3 .. Moreover, if sealed bid auctions are essentially static, the dynamic nature of English and Dutch auctions has to be stressed. Therefore, English auctions (respectively Dutch auctions) are often referred to Ascending price auctions (respectively Descending price auctions).

Of course, many variations of the previous descriptions can be found in the literature (108). In Japanese auctions all the bids are made at the same time, orally and publicly. In Candle auctions, bids are accepted as long as a candle remains lit. For instance, a variant of the English auctions is the following: the auctioneer starts the auction by calling out a low price. Then he continuously increases the price. The bidders have their hand raised as long as they are still interested to buy the item at the current called price. The auction stops as soon as there is only one bidder with his hand raised left. As a consequence, the winning bidder pays an amount equal to the price at which the second-last bidder dropped out (67).

At this point, it is worth noting that the terminology presented in this

\footnotetext{
${ }^{2}$ i.e. a price that is high enough to be sure that no bidder wants to buy the item at that price.

${ }^{3}$ some authors (115) further distinguish semi-sealed bids if partial information about the offers is communicated to the bidders
} 
chapter is commonly used in the academic literature. Nevertheless, in practice, some denominations can vary considerably. For instance, Dutch auctions are sometimes (41) referred to as multi-unit auctions (see section 1.4).

Furthermore, even when a specific type of auction has been chosen, a number of additional parameters are likely to influence its execution and so, its outcomes. Among them, let us mention:

- Bid increment

A bid increment is the minimum acceptable price difference between two successive bids that is accepted.

- Reserve Price

In many cases, the seller fixes a (public or private) reserve price i.e. a minimum price that has to be reached at the end of the auction to proceed with the selling. If the final price is lower than the reserve price, the seller has the right not to sell the item.

- Buyout price

If, during the auction, a bid greater of equal than the buyout price is received, the auction automatically stops and the item is sold to the bidder who proposed this offer.

- Closing rules

Many different closing rules can be considered. For instance, in English auctions, the description above illustrates a closing rule that is based on the bidding activity. Another closing rule could be to fix a closing time, i.e. the auction stops Thursday at noon.

- Entry fee

Bidders have to pay an entry fee in order to be allowed to participate to the auction. In the same spirit, in some cases, only pre-selected (or invited) bidders are allowed to participate to the auction (open participation or by invitation).

Regarding all these different types of auctions, two fundamental questions (75) are raised:

- Why is an auction format used rather than some other selling (or buying) procedure? 
- Given the diversity of types of auctions, what determines which particular auction form is chosen?

Auctions offer the possibility to dynamically fix prices on the basis of (relatively) simple information exchanges: bids from market participants.

First of all, the value of certain goods is highly volatile. The price of electricity on the Amsterdam Power Exchange (APX), for instance, may vary considerably with respect to changes in the demand and supply conditions. Perishable goods constitute other examples for which the value rapidly evolve. In such contexts, the dynamic aspect of auctions offers an elegant way to quickly adjust prices.

Moreover, some products have no standard value (24). Indeed, in some cases, the price of specific goods is difficult to precisely estimate. The value of a manuscript form Leornardo Da Vinci ${ }^{4}$ is such an example. In privatization contexts, the value of national company constitute another example. In such cases, by using an auction, these values will be determined by the market (i.e. bids from market participants).

To address the second question, researchers working in auction theory have built theoretical models. One of the them, the independent private values model, will be briefly outlined in the next section.

\subsection{The Independent Private Values model}

When modeling auctions, four basic issues are usually considered (89):

- Are the bidders (or the seller) risk neutral or risk averse ?

- How are the bidders' valuation of the object? Independent or correlated?

In auction theory, the bidders' valuation of the item are assumed to be private information: this is the main difficulty associated to the management of auctions! The independent values assumption refers to the case where the valuation of one bidder does not depend on the valuation of another bidder. This happens, for instance, with consumption goods (104): the bidder is not willing to pay more (or less) because another

\footnotetext{
${ }^{4}$ the so-called codex hammer was sold by Christie's in 1994 for a record amount of 30.802 .500 dollars
} 
bidder values the good differently (since, anyway, he will consume the good). This is, of course, an idealized context. Most of items sold using auctions such as paintings, financial products, ... do not respect this assumption. If a bidder $s_{i}$ knows that another bidder $s_{j}$ values the item higher, $s_{i}$ is likely to increase his valuation. Anyway he will still realize a margin if he re-sales the item afterwards. This situation is referred to as the affiliated values assumption. An extreme case is the common values assumption: the item sold has a single value denoted $V$. Therefore, the bidders valuation are strongly dependent. This happens, for instance, with the auction of mineral rights.

- Are the bidders identical or heterogeneous ? The first case will be referred to symmetric bidders (versus asymmetric bidders)

In some cases, bidders are not homogeneous. For instance, when a building contract is auctioned, some foreign contractors have cheaper labor price than national ones.

- Does the final payment depend on other variables than solely the bid ? In all the mechanisms introduced above, the final payment is solely determined by the bid. However, in some cases, the final payment may depend on other variables. For instance, in auctions of publishing rights (89), the final payment depends both on the final bids but also on royalties based on the realized sales of the book. Another example based on the building of highways will be presented in chapter 3 .

The model of Independent Private Values (IPV) relies on the easiest modeling issues: bidders are assumed to be symmetric and risk neutral. Their valuation are independent and the final payment only depends on the bids submitted. This model is treated in the literature as a benchmark model.

First of all, let us remark that Dutch auctions are equivalent to first price sealed bid auctions. Indeed, when a bidder participates to a Dutch auction he has to fix (before the auction beginning) the price level at which he will react during the auction. This strategy is exactly the same as the one used in a first price sealed bid auction (67).

Moreover, English auctions are equivalent to Second Price Sealed Bid auctions.

- On the one hand, a dominant strategy ${ }^{5}$ for the bidders participating

\footnotetext{
5intuitively, a strategy that a bidder is better to apply whatever the other bidders' strategy.
} 
in an English auction is to continue to bid until they have reached their valuation. As a consequence, the winning bidder in an English auction is the bidder with the highest valuation and the winning bid is equal to the second highest valuation.

- On the other hand, a dominant strategy in a second price sealed bid auction is to propose a bid equal to the valuation. Indeed, the bid communicated in a Vickrey auction only influences the probability of winning, the winning bid being determined as being equal to the second highest bid. Therefore, bidding lower than the valuation decreases the probability of winning without increasing the margin. On the contrary, bidding higher than the valuation may lead to situations where the winning bid is higher than the valuation and thus to negative margins. As a consequence, the winning bidder in a Vickrey auction is the one with the highest valuation and the winning bid is equal to the second highest valuation.

Let us note that, if the valuations are not independent, this equivalence is not true anymore since, during an English auction, bidders may learn some information about the others' valuations.

A major result of auction theory is known as the revenue equivalence theorem. This gives a first answer to the second fundamental question presented in the previous section.

Theorem 1 Revenue Equivalence Theorem (124; 81; 92; 75)

For the benchmark model, each of the English auction, the Dutch auction, the first-price sealed-bid auction, the second-price sealed bid auction yields the same price on average.

Of course, the assumptions underlying the benchmark model are rather restrictive and a number of experimental analyses has shown its limits. We refer the interested reader to the book of Kagel and Roth (59) which reviews a number of experimental analysis.

As already stressed, the Independent Private Values model is a benchmark model in auction theory. Many other traditional models may be considered. Among others let us cite the common value model (78) or the correlated values model (75). We refer the interested reader to (64) for their detailed analysis. On the other hand, more recently, a number of extensions of classic auction mechanisms have been proposed. Some of them are described in the next section. 


\subsection{A few selected extensions}

In traditional auction theory, one basically distinguishes two situations:

- There is one item to sell and several potential buyers in competition. This situation is referred to as a forward auction.

- There is one item to buy and several potential sellers in competition. This situation is referred to as a reverse auction

Nevertheless, as shown here after, these situations have been (recently) extended to more general settings, namely double, multi-unit or multi-object auctions.

In double auctions (49), both several buyers and several sellers interact. This kind of situation happens, for instance, in financial markets where, simultaneously, several bidders propose to sell different quantities of a specific asset for different prices and several bidders propose to buy different quantities of this asset at different prices. The winner determination is based on the market clearing price, i.e. the price that is at the intersection between the aggregated supply and demand curves.

In multi-unit auctions, several units of an homogeneous good are sold. A first approach could be to successively sell the different units using a traditional auction. However, if there are $m$ units to sell, $m$ different auctions have to be organized! Furthermore, in such a context, the bidders' strategy is not obvious: at each step, they have to evaluate the chance they have to acquire one item for a lower price in the remaining auctions. A second approach is thus to simultaneously sell the $m$ units. When the auction stops, the winning bidders are those who have proposed the $m$ highest prices. Two distinct situations can happen concerning the final price paid by the winning bidders. Either each winning bidder pays the amount he proposed (price discrimination, this is also referred to as Yankee auctions (60)) or all the winning bidders pay the same price i.e. the one corresponding to the $m^{\text {th }}$ highest bid (or the $(m+1)^{\text {th }}$ highest bid). In multi-unit auctions, bidders propose offers that are characterized by a pair (price,quantity) i.e. the quantity they are ready to buy at a specific price. Therefore, they are able to express their preferences among these couples. As stressed by Klemperer (64), multi-unit auctions constitute one of the most active research direction within auction theory. We refer the interested reader to (67) for a detailed description of the field. 
In multi-object auctions ${ }^{6}$, several heterogeneous items are simultaneously sold. Therefore, bidders are allowed to bid, not only on each item, but also on bundle of items. By doing so, they are able to express synergies and redundancies between the items (in other words, the bidders can express their preferences according to different bundles of items, see example 1).

Example 1 FCC spectrum right auctions

When introducing multi-object auctions, most authors refer to the FCC spectrum right auctions. It is, indeed, a particularly well-adapted context to illustrate the need for bundle bids.

Let us consider 5 different states, denoted $A, B, \ldots, E$ that simultaneously propose licenses for the third generation of mobile phones ( $L X$ denotes the license of state $X)$. Let $b_{i}(S)$ be the bid proposed by bidder $s_{i}$ for a subset of licenses $S$. In this case, we will have $b_{i}(L A \cup L B) \geq b_{i}(L A)+b_{i}(L B)$, i.e. bidder $s_{i}$ is ready to pay more if he is sure to simultaneously get both $L A$ and $L B$ than the sum of individual bids. This is due to the fact that $A$ and $B$ are neighbouring states (see figure 1.1). On the other hand, bidder $s_{i}$ has no interest to simultaneously get licenses for distant states. Therefore, $b_{i}(L A \cup L C) \leq b_{i}(L A)+b_{i}(L C)$.

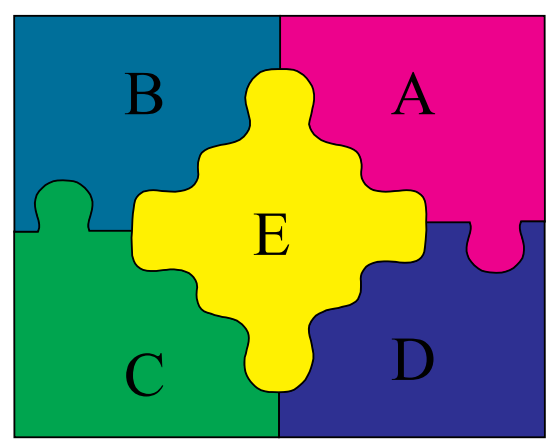

Figure 1.1: The selling of UMTS licenses: the example of 5 neighbouring states.

When using multi-object auctions, a major concern is the determination of the optimal allocation. This problem is referred to the Combinatorial Auction Problem (CAP). Let $M=\left\{o_{1}, \ldots, o_{m}\right\}$ the set of $m$ objects that are simultaneously sold and let $S \subseteq M$ be a bundle of objects. Let $N=$ $\left\{e_{1}, \ldots, e_{n}\right\}$ be the set of bidders participating in the auction. If $b_{j}(S)$ denotes

\footnotetext{
(128)

${ }^{6}$ also referred as combinatorial auctions $(94 ; 105 ; 83)$ or multi-dimensional auctions
} 
the best bid offered by the $j^{\text {th }}$ buyer for $S$, the CAP can be formalized as follows:

$$
\begin{gathered}
\max \sum_{j \in N} \sum_{S \subseteq M} b_{j}(S) \cdot y(S, j) \\
\sum_{S \subseteq M \mid o_{i} \in S} \sum_{j \in N} y(S, j) \leq 1, \forall o_{i} \in M \\
\sum_{S \subseteq M} y(S, j) \leq 1, \forall j \in N \\
y(S, j) \in\{0,1\}, \forall S \subseteq M, j \in N
\end{gathered}
$$

where $y(S, j)$ is a binary variable such that $y(S, j)=1$ implies that, in the optimal allocation (i.e. the one maximizing the seller's revenue), the bundle $S$ is allocated to the $j^{\text {th }}$ bidder at a price $b_{j}(S)$. The first constraint imposes that each object is allocated to, at most, one bidder. The second constraint implies that, at most, one bundle is allocated to each bidder (the bidders are assumed to propose bids for every bundles of objects). This problem is known as a set packing problem ${ }^{7}$. Several authors have studied its resolution in the particular context of auctions. We refer the interested reader to $(94 ; 105 ; 83)$ for a detailed analysis. Finally, let us note that if the bids are divisible i.e. if partial quantities are acceptable, the variable $y(S, j)$ are not boolean anymore and are interpreted as the proportion of the bid that is allocated to bidder $e_{j}$.

Of course, since bidders are allowed to propose bids for every bundles of objects (i.e. for a total of $2^{m}-1$ different bundles), the expressivity of the bids is a key issue. Therefore, some authors (83) have studied bidding languages.

Furthermore, in this context, the cognitive complexity of the bidding process increases and bidding strategies are difficult to establish. As pointed by (115), most of the research done in multi-object auctions has been focused on the CAP (i.e. a support for the auctioneer). Nevertheless, on the bidders side, bidding support tools are also needed.

\footnotetext{
${ }^{7}$ which is NP-complete
} 
As shown in chapter 3, combinations of the previous approaches, such as multi-unit multi-objects auctions, can be considered. Furthermore, additional constraints can intervene in the model. For instance, in the context of a reverse multi-unit auction, a constraint could be: the auctioneer must buy the desired quantity of items from at least three different suppliers (in order to avoid a too strong dependence to a unique supplier).

These few extensions demonstrate how rich and active is the field of auction theory. This will be further completed with chapter 3 which is about multi-attribute auctions.

\section{5 e-Auctions}

Auctions have been recently applied to e-Commerce with success stories such as eBay (41), Onsale (86) ${ }^{8}$, uBid (119), Yahoo auctions (129), Amazon auctions (2), ... No doubt that these web sites have deeply stimulated the general public's interest about auctions allowing nearly everybody to use them to buy or sell things.

Historically, David Lucking-Reiley (70) has been one of first authors to analyze this phenomenon. In the article "Auctions on the Internet: What's Being Auctioned and How ?", he described the "e-Auctions landscape" in August 1998 on the basis of 142 web sites. Here after, we summarize some of his observations. This will be completed by an outline of eBay in section 1.5.1.

e-Auctions web sites are quite diverse in nature. Some of them, such as AuctionVine (7) are specialized in auctioning a specific kind of goods (in this case wine) while others, such as Yahoo auctions, host a large number of distinct categories. Some web sites are essentially trading their own goods, such as Onsale for instance (these are referred to Merchant sites) while others, like eBay, works as listing-agent sites. Finally, some web sites, such as InternetAuctionList (55), work as auctions portals, i.e. searching for auctions on different e-Auctions web sites. As for traditional auctions, the English auction format is the most represented.

e-Auctions, compared to traditional auctions, offer a number of advantages. First of all, costs related to the organization and management of auctions are deeply lowered. For instance, in auction houses like Sotheby's,

${ }^{8}$ which was apparently (70) the first e-Auctions web site (opened in May 1995) 
the commission is paid by both the seller (20\% of the announced price) and the buyer ( $15 \%$ of the final price). When a seller uses eBay, he only has to pay between 0.25 and 2 dollars to be allowed to start an auction. Once the object is sold, he has to pay a commission of approximately $5 \%$ on the final price. Moreover, due to the geographic independence (bidders do not have to gather at the same place) and time flexibility (auctions last usually longer than classic auctions), a larger public can participate to the auction and a larger choice of items is available.

Most of e-Auctions last several days. eBay for instance proposes 4 distinct length: 3, 5, 7 or 10 days. Shorter periods, such as express auctions (60 minutes) or flash auctions (3 minutes) can also be found. The longest auction in the survey (70) lasted about 90 days. Most of e-Auctions use a predefined closing rule (i.e. the auction stops, for instance, Thursday at noon). As a consequence, one observes that the bidders wait the last minutes to send their bids (this is referred to snipping see below). To counter this behavior, some web sites use the following rule: if an abnormally high activity is detected during the last minutes before the auction's end, the length of the auction is extended with a specific delay.

Of course, with the emergence of e-Auctions, a number of new behaviors have appeared or, at least, have been re-enforced. Among them we can cite (29):

- snipping: the act of bidding at the very last possible second of an auction

- bid shielding: an illegal process wherein two bidders work together to defraud a seller out of high bids by retracting a bid at the last minute and granting a confederate's low bid the win.

- shill bidding:the practice of placing a bid on an item to artificially inflate the final value.

Finally, from a technological perspective, auctions have also received great attention within the multi-agent field $(102 ; 104 ; 93)$ : they have been used for resources and tasks allocation between computerized agents (93) and electronic auction platforms like AuctionBot (127), eMediator (103) and FishMarket (84) have been built with the option to support agents interactions. 


\subsubsection{The case of eBay}

Speaking about e-Auctions, without speaking about eBay ${ }^{9}$ is probably like speaking about Physics without citing Newton! The success of eBay is worldwide and has overcame most of analysts' expectations. For instance, nowadays in the USA, a car is sold on eBay every minute (on average) (42). Selling or buying on eBay is, for some people, a full-time professional activity. The so-called eBay PowerSellers have a gross monthly volume at least greater than $1.000 \$$ (Bronze level), 3.000\$ (Silver), 10.000\$ (Gold level), $25.000 \$$ (Platinum level) or 50.000\$ (Titanium level) (29). Furthermore, famous brands such as Nike, HP or Motorola have their official eBay stores. Finally, searching on Amazon.com about books on eBay leads to more than 350 titles (most of them explaining the best strategy to win in online auctions).

\begin{tabular}{c|ccc} 
& Q2 2004 & Q2 2005 & Evolution \\
\hline Consolidated net revenues & $773.4 \$$ & 1.086 billion $\$$ & $+40 \%$ \\
Active users & 48 million & 64.6 million & $+34 \%$ \\
Gross Merchandise volume & 8 billion $\$$ & 10.9 billion $\$$ billion & $+36 \%$ \\
Listings & 332.3 million & 440.1 million & $+32 \%$ \\
\hline
\end{tabular}

Table 1.1: Statistics and recent evolution of eBay - Second Quarter 2004 and 2005 (41)

Since the creation of eBay in September 1995, the number of users, listed items and other related activity factors haven't stopped increasing. Table 1.1 further illustrates this assertion. Nowadays, eBay offers a wide variety of services to its clients. Two of them are outlined hereafter.

First of all, when trading on the Internet, your interlocutors are humans beings (or at least software agents that represent human beings) that hide themselves behind an ID or a nickname. Even if a nickname sounds great, you never know if the person behind it will behave as a reliable commercial partner or not. To reduce the uncertainty related to encounters, eBay proposes a feedback program that gives information about the buyer or the seller behavior (number of bid retractions during the past 6 months, positive, neutral or negative feedbacks about previous trades, ...).

Moreover, since e-Auctions last several days, and that buyers cannot stay

\footnotetext{
${ }^{9}$ If eBay is mainly focused on e-Auctions, it also proposes live auctions (43) or fixedprice sales
} 
online the whole day to follow the auctions they participate, eBay proposes that an artificial agents bids on behalf of the user. This is referred to proxy bidding. In practice, the user specifies the maximum amount he is ready to pay for the considered item and the agent automatically outbids offers from other buyers until he reaches this amount.

To conclude, let us stress that eBay remains an evolving web site that is more and more analyzed by the academic world $(54 ; 90 ; 61)$.

\subsection{A global perspective}

As shown in the previous sections, auction theory is a rich research sector that is in constant evolution. In order to give the reader a global perspective of the field, we present, here below, a classification of auction situations based on their characteristics (see table 1.2) that has been recently proposed by Teich et al. (115). Most of the characteristics listed in table 1.2 have been introduced in the previous sections. Some others $(4,13,14$ and 15) are related to multi-attribute auctions and so will be introduced or further explained in chapter 3 .

\subsection{Conclusion}

In this chapter, our aim was both to introduce the basic vocabulary related to auction theory and to illustrate the richness of this field. This will be further completed by chapter 3 which is about multi-attribute auctions.

If the first works on traditional auctions were essentially studied by means of probabilistic and statistical tools, extensions such as multi-unit or multiobject auctions rely more and more on operations research tools. This trend will be further marked with multi-attribute auctions. 


\begin{tabular}{|lc|}
\hline Characteristic & Range \\
\hline 1. Number of items of a certain good & One to many \\
2. Number of goods auctioned & One to many \\
3. Nature of goods & One to many \\
4. Attributes & Reverse vs forward \\
5. Type of auction & One round vs progressive \\
6. Nature of auction & Ascending, descending price \\
7. English versus Dutch auction & By invitation vs open \\
8. Participation & Agent mediated vs manual mode \\
9. Use of agents & First price vs second price vs $n^{\text {th }}$ price \\
10. Price paid by winner & Yes, no \\
11. Price discrimination & Implicitly, explicitly \\
12. Constraint exist & Yes, no \\
13. Follow-up negotiation & Yes, no \\
14. Value function elicitation & Open cry vs semi-sealed vs sealed \\
15. Nature of bids & 1,2, or n-dimensional \\
16. Bid vector & Yes, no \\
17. Bids divisible & Yes, no \\
18. Bundle bids allowed & \\
\hline
\end{tabular}

Table 1.2: Classification of auction situations based on their characteristics 


\section{Chapter 2}

\section{Multicriteria Decision Aid}

\section{$2.1 \quad$ Introduction and motivations}

Multicriteria Decision Aid (MCDA) has been an active field of research for more than 30 years. Summarizing it in a few pages is, of course, impossible. Consequently, the only ambition of this chapter is to constitute a rough introduction to the subject.

As shown here after, a brief analysis of the terms "multicriteria decision aid" already allows the novice to understand the underlying motivations of this research area (35). It is, first of all, a decision aid activity (versus decision making) that has its root in the multicriteria paradigm. These statements will be further commented in the next two subsections. We refer the interested reader to $(99 ; 98 ; 122 ; 8 ; 120 ; 17 ; 106 ; 35)$ for detailed discussions.

The rest of the chapter is organized as follows. In section 2.2, we will introduce the basic terminology and notions related to multicriteria decision aid. This will lead us to define multicriteria problems. Then, two distinct multicriteria approaches will be outlined: multi-attribute utility functions (in section 2.3.1) and outranking methods (in section 2.3.2). In the latter subsection, both the Electre and Promethee methods will be outlined. Then, a few selected modeling issues will be presented in section 2.3.3. Finally, general additional comments and concluding remarks will end this chapter.

At last, in order to illustrate the notions that are introduced in the following sections, we will constantly refer to the following example (taken from 
the field of multi-attribute auctions):

Example 2 The cafeteria problem.

A company wants to sub-contract the activities related to her cafeteria. Therefore, it decides to organize a procurement auction. The potential suppliers are invited to submit offers for a one-year contract (250 working days - 500 meals a day).

\subsubsection{Decision aid}

Selecting an investment project, appointing a new employee, choosing a site to establish a garbage dump, diagnosing a disease, ... All these examples show that deciding is a complex activity that, in many cases, can have important consequences.

A decision is, first of all, the result of a more or less time consuming process that is made up partial decisions, negotiations and learning phases, search for (additional) information, ... During this process, new potential solutions can appear while others become not topical anymore. The context of the problem can be such that the evaluation of the potential solutions has to be made according to several conflicting point of views (eventually integrating subjective elements). The related data are often imprecise, uncertain or simply not available. Social, economic and political constraints further increase the complexity of the decision process. Finally, most of decisions involve different actors with different value systems.

Facing the complexity of this activity, one may try to build a model, i.e. an abstraction of the reality that will be used, during the decision process, as a support for the investigations and communications. The limited, approximate and imperfect nature of this model has to remind us of his modesty. This observation has led Bernard Roy (99) to define decision aid as follows:

Definition 1 Decision aiding is the activity of the person who, through the use of explicit but not necessary completely formalized models, helps obtain elements of responses to the questions posed by a stakeholder ${ }^{1}$ in a decision process. These elements work towards clarifying the decision and usually towards recommending, or simply favoring, a behavior that will increase the

\footnotetext{
${ }^{1}$ here, the term stakeholder refers to any individual or entity that intervene in the decision making process.
} 
consistency between the evolution of the process and this stakeholder's objectives and value system.

\subsubsection{The multicriteria paradigm}

In the fifties, the pioneers of operational research were convinced of the natural and promising applicability of their models. Twenty years later, the reality was somewhat different: some problems had been successively treated by using classic operational research tools while, in other cases, their application had disappointed.

As remarked by Schärlig (106), the success stories were essentially related to situations where the decision problem could have been isolated from their context: the search for optimal mixtures, an optimal traveling problem, an optimal stock management, ... In the other cases, the underlying assumptions of classic OR models appeared to be too restrictive to constitute an adequate model of the reality.

Indeed, most of unicriterion optimization approaches rely on the following (implicit) assumptions (106):

- stable set of actions: the set of alternatives is assumed to be known prior to the analysis and to remain unchanged during the decision process. On contrary, in most decision problems, new alternatives can appear during the analysis while others become not topical anymore.

- exclusive actions: every alternative is assumed to perfectly reflect all the facets of the problem.

- transitivity assumption: the preferences of the decision maker are assumed to be transitive. This hypothesis has been severely criticized (see below). In many real life problems, nor the (strict) preference nor the indifference relations are transitive.

Among the critics listed above, the one related to the non-transitivity of preferences is definitively the most crucial. This distinctive feature comes (at least partially) from the fact that most of decision problems involve several conflicting criteria (while the classic OR models are built to optimize a unique criterion under several constraints). As a consequence, the notion of optimal 
solution ${ }^{2}$ is not meaningful anymore. Therefore, in multicriteria contexts, researchers will rather consider compromise solutions, i.e. solutions that are "globally good" according to the different criteria (without necessarily being the best for a given criterion) and that are not too bad for any given criterion.

In unicriterion optimization models, the apparent universality of the $o p$ timal solution concept leads the analyst ${ }^{3}$ to search for an hidden truth $(106 ; 123 ; 35 ; 99)$. On the contrary, the arguments presented above (and in the previous subsection) lead to acknowledge that the objectives of multicriteria decision aid are much more modest. It is, first of all, a structuring process that leads to search for satisfactory solutions with respect to the decision maker's values.

\subsection{Basic terminology and problem's formula- tion}

Facing the complexity of a decision problem, the Decision Maker (DM) tries to rationalize it. Therefore, he has to identify the key elements that will intervene in the decision process, i.e. the object of the decision, the set of potential solutions, a way to evaluate and compare them, the factors that can influence the decision(s), ... This structuration phase is at the core of the multicriteria decision aid activity.

In this section, we will introduce the basic terminology that is used within the MCDA community and, consequently, increase the reader's awareness of the MCDA problem's formulation.

\subsubsection{The set of actions and their consequences}

At first, let us introduce the notion of an action. Intuitively, the actions are the set of objects, alternatives, items, candidates, projects, potential decisions, ... on which the decision is based. More formally,

\footnotetext{
${ }^{2}$ In most cases, an alternative that is optimal for a specific criteria will not be optimal for another criteria (on the contrary, it is likely to be a bad solution according to this second point of view). In fact, most of people interpret the term optimal solution in an erroneous way because they assign it a global meaning. On the contrary, in practice, one should ask the question optimal with respect to which criteria?

${ }^{3}$ i.e. the person that helps the decision maker during the decision process
} 
Definition 2 (100) An action is a generic term used to designate that which constitutes the object of the decision, or that which decision aiding is directed towards.

In what follows, we will denote the set of actions $\mathcal{A}=\left\{a_{1}, \ldots, a_{p}\right\}$. As stressed by Vincke (123), $\mathcal{A}$ can be:

- stable: if $\mathcal{A}$ can be defined a priori and is not likely to change during the decision process

- evolutive: if, on the contrary, $\mathcal{A}$ is likely to change during the decision process. Indeed, the decision process being an dynamic activity, intermediary results and / or the potential evolution of the decision context can lead to consider new actions while others are not topical anymore.

Furthermore, let us stress that $\mathcal{A}$ is said to be globalized, if each element of $\mathcal{A}$ excludes any others, and fragmented if it is not the case i.e. if combinations of elements from $\mathcal{A}$ constitute possible issues of the decision process. Finally, one generally distinguishes contexts where $\mathcal{A}$ can be defined by extension (the cardinal of $\mathcal{A}$ is finite and relatively small. As a consequence, its elements can be enumerated) and situations where it is defined by comprehension (the cardinal of $\mathcal{A}$ is infinite or relatively high. The elements of $\mathcal{A}$ are identified as those satisfying a set of specific constraints).

Once the set $\mathcal{A}$ has been determined, one has to characterize the actions (according to different points of views). This is formalized by the criterion's notion.

Definition 3 (123) A criterion is a function $\xi$, defined on $\mathcal{A}$ taking its value in a totally ordered set and representing the decision maker's preferences according to some point of view.

$$
\xi: \mathcal{A} \rightarrow \text { E where } E \text { is a totally ordered set }
$$

Let us remind the reader that, in the present work, we will assume that all the criteria have to be minimized. Let $\xi^{j}\left(a_{i}\right)$ denote the evaluation of action $a_{i}$ according to the criterion $\xi^{j}$. Let us assume that $q$ distinct criteria are involved in the decision problem. In what follows, we will alternatively use the term criterion or attribute. 
At this point of the analysis, the only objective information that can be extracted from the decision problem is based on the Pareto dominance relation:

Definition 4 Let $\prec$ denotes the Pareto dominance relation i.e. $\quad b \prec \tilde{b} \Leftrightarrow$ $\xi(b)^{j} \leq \xi(\tilde{b})^{j} \forall j \in\{1, \ldots, q\}$ and $\exists \tilde{j} \in\{1, \ldots, q\} \mid \xi(b)^{\tilde{j}}<\xi(\tilde{b})^{\tilde{j}}$. Similarly, we define $\preceq$ as follows: $b \preceq \tilde{b} \Leftrightarrow \xi(b)^{j} \leq \xi(\tilde{b})^{j} \forall j \in\{1, \ldots, q\}$

The exploitation of this relation leads to distinguish efficient and dominated actions from $\mathcal{A}$.

Definition 5 An action $a$ is said to be efficient if $\nexists \tilde{a} \in \mathcal{A}: \tilde{a} \prec a$

Nevertheless, most of the time, once the dominated actions have been removed from $\mathcal{A}$, the number of remaining efficient actions is still important since, most of the time, there is no action that is simultaneously the best for all the criteria. On the other hand, one can explicitly build a virtual action, called ideal point, that satisfies the aforementioned condition:

Definition 6 The ideal point, $i(\mathcal{A})$, associated to $\mathcal{A}$ is the point the coordinates of which are $\left(i(\mathcal{A})^{1}, \ldots, i(\mathcal{A})^{q}\right)$ where:

$$
i(\mathcal{A})^{j}=\min _{a \in \mathcal{A}} \xi^{j}(a)
$$

Since the ideal point (or assimilated actions, i.e. that are the best for all criteria) does not usually belong to $\mathcal{A}$, the notion of optimal solution is not adapted to multicriteria problems. On the contrary, in most cases, the presence of conflicting criteria will lead the decision maker to rather focus on compromises solutions. Therefore, the preferences of the decision maker have to be modelled and exploited.

Example 3 The cafeteria problem.

The buyer, i.e. the manager of the company who is in charge of the procurement, decides to evaluate the offers according to 3 criteria:

- $\xi^{1}$ : the price of the yearly contract. The reserve price for a daily meal being equal to 12,5 Euros, the value of $\xi^{1}$ will never exceed 1.562 .500 Euros $(=12,5 \cdot 250 \cdot 500)$. In other words, $E^{1}=[0$ 1.562.000]. 
- $\xi^{2}$ : the meals variety. This criterion expresses the number of distinct meals a day that are proposed to the employees. The values of this criterion range from 1 to 3 .

- $\xi^{3}$ : the meals quality. In addition to $\xi^{2}$, the buyer decides to distinguish the offers in terms of the meal quality. Therefore, the buyer defines three quality levels $A, B, C$ ( $A$ being better than $B$ and $B$ being better than $C)$. For instance, the quality level $A$ correspond to meals that are composed of a starter, main dish and dessert while meals corresponding to level $B$ are only composed of a soup and a main dish, ....

During the auction, the buyer will receive a succession of offers from the suppliers that will constitute the set of actions. Consequently, in this context, $\mathcal{A}$ is evolutive and defined by extension. The first offer received by the buyer is $(685.0001 C)$; i.e. a low price, low quality bid.

Once the set of actions and the set of criteria have been determined, one still has to analyze the decision maker's preferences. This step will permit to build a model that will, as much as possible, correctly represent his decision behavior.

\subsubsection{Preference modelling}

When modelling the decision maker's preferences, one usually distinguishes the three following binary relations ${ }^{4}$ : Preference $(\mathrm{P})$, Indifference $(\mathrm{I})$, and Incomparability $(\mathrm{J})$, which result from the comparison between two actions $a_{i}$ and $a_{j} \in \mathcal{A}$

$$
\begin{cases}a_{i} P a_{j} & \text { if } a_{i} \text { is preferred to } a_{j} \\ a_{i} I a_{j} & \text { if } a_{i} \text { is indifferent to } a_{j} \\ a_{i} J a_{j} & \text { if } a_{i} \text { is incomparable to } a_{j}\end{cases}
$$

Indeed, these relations translate situations of preference, indifference and incomparability and it can be assumed that they satisfy the following requirements:

\footnotetext{
${ }^{4} R$ is a binary relation on $\mathcal{A} \Leftrightarrow R \subseteq\left\{\left(a_{i}, a_{j}\right) \mid a_{i}, a_{j} \in \mathcal{A}\right\}$
} 


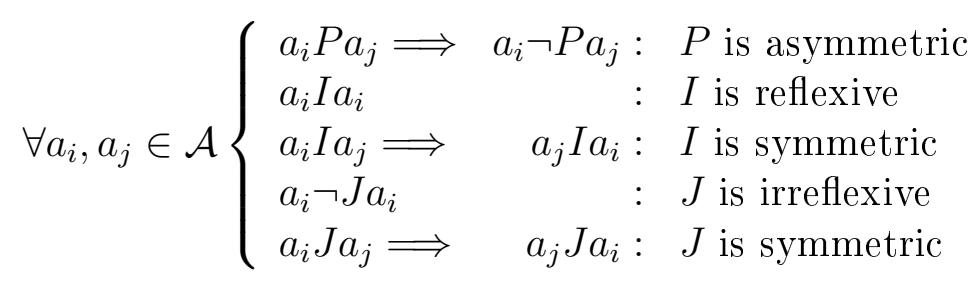

Definition 7 ((123)) The three relations $\{P, I, J\}$ make up a preference structure on $\mathcal{A}$ if they satisfy the above conditions and if, given any two elements $a_{i}, a_{j}$ of $\mathcal{A}$, one and only one of the following properties is true: $a_{i} P a_{j}, a_{j} P a_{i}, a_{i} I a_{j}, a_{i} J a_{j}$.

Intuitively (99):

- $a P b$ corresponds to the existence of clear and positive reasons that justify significant preference in favor of a;

- $a I b$ corresponds to the existence of clear and positive reasons that justify equivalence between the two actions;

- $a J b$ corresponds to an absence of clear and positive reasons that justify any of the two preceding relations.

In the classic unicriterion optimization models, the pair wise comparisons of actions can only lead to two situations: preference or indifference. In the same way, many multicriteria methods, such as multi-attribute utility functions for instance, aggregate all the criteria into a unique (artificial) value and, therefore, transform the multicriteria problem into a unicriterion optimization problem. In these contexts, both the indifference and preference relations are assumed to be transitive. These hypothesis have, nevertheless, been criticized by several authors. For example, Luce (69) illustrates the non-transitivity of the indifference relation with the following example: let us consider 401 cups of coffee noted $C_{0}, C_{1}, \ldots, C_{400}$. One assume that the cup $C_{i}$ contains exactly $\left(1+\frac{i}{100}\right)$ grams of sugar. In this context, any normal person is unable to differentiate two successive cups. Therefore, we have: $C_{O} I C_{1}, C_{1} I C_{2}, C_{2} I C_{3}, \ldots, C_{399} I C_{400}$. Nevertheless, it is obvious that nobody will state $C_{0} I C_{400}$. In the same way, the next example illustrates a situation where the preference relation is not transitive.

Example 4 The cafeteria problem

As already stressed, preferences are not always transitive. In order to illustrate this phenomenon let us consider the following context: the manager 
estimates that all the criteria are equally important and doesn't want to base his decision on a complex model. On the contrary, he assumes that an offer $b_{1}$ is preferred to another offer $b_{2}$ as soon as $b_{1}$ is better than $b_{2}$ for at least a majority of the criteria (i.e. for at least 2 criteria - we assume here that the criteria are equally important). Applying this rule to the data listed in table 2.1, leads to $b_{1} P b_{2}, b_{2} P b_{3}$ and $b_{3} P b_{1}$ (this is also referred to as the condorcet effect (47)).

Table 2.1: Example of condorcet effect.

\begin{tabular}{|c|ccc|}
\hline Bids & Variety & Quality & Price \\
\hline$b_{1}$ & 2 & $\mathrm{C}$ & 1.000 .000 \\
$b_{2}$ & 1 & $\mathrm{~A}$ & 1.150 .000 \\
$b_{3}$ & 3 & $\mathrm{~B}$ & 1.300 .000 \\
\hline
\end{tabular}

Let us note that some authors (99) further enriches the previous structure by a $Q$ relation which stands for a weak preference relation (versus the strict preference relation $P$ ). In other words, if $a_{i} Q a_{j}$, the decision maker knows that $a_{j} \neg P a_{i}$ but cannot clearly choose between $a_{i} I a_{j}$ or $a_{i} P a_{j}$. This specific relation will not be considered in the present work.

The presence of potential incomparability is a distinctive feature of the so-called French school of multicriteria decision aid. As already stressed, $a \mathrm{Jb}$ is stated when the decision maker cannot clearly choose among the three following possibilities: $a P b, b P a$ or $a I b$. This can happen, for instance, due to (47) a lack of information, uncertainty or conflicting preferences (see (99) for illustrative examples).

Finally, in what follows, we will often use the relation $\mathcal{S}=(P \cup I)$. Thus, $a_{i} \mathcal{S} a_{j}$ will stand for $a_{i}$ is at least as good as $a_{j}$. A direct consequence of this definition is:

$$
\forall a_{i}, a_{j} \in A \begin{cases}a_{i} P a_{j} \Leftrightarrow & a_{i} \mathcal{S} a_{j}, a_{j} \neg \mathcal{S} a_{i} \\ a_{i} I a_{j} \Leftrightarrow & a_{i} \mathcal{S} a_{j}, a_{j} \mathcal{S} a_{i} \\ a_{i} J a_{j} \Leftrightarrow & a_{i} \neg \mathcal{S} a_{j}, a_{j} \neg \mathcal{S} a_{i}\end{cases}
$$

We refer the interested reader to (16) for a detailed introduction about binary relations and preference modeling.

Until now, we have restricted ourselves on binary relations for preference modelling. Let us note that another important trend relies on valued rela- 
tions. This will be illustrated in section 2.3.2.2 which is about the Promethee method.

\subsubsection{Consistent family of criteria}

A fundamental problem in multicriteria decision aid is to represent the decision maker's preferences on the basis of the evaluations of the actions, $a_{i} \in \mathcal{A}$, according to the different criteria involved in the problem. The selection of these criteria is thus a crucial first step of the modeling activity. One way to formalize this selection is to require certain properties such as exhaustivity, cohesion and non redundancy. Intuitively:

- exhaustivity: if $a_{i}$ and $a_{j}$ are two actions that are identical with respect to all criteria, then one cannot have $a_{i} P a_{j}$ or $a_{j} P a_{i}$. If it is the case then there exists another criterion differentiating $a_{i}$ from $a_{j}$ that has not been considered. This criterion should then be added to the family of criteria.

- cohesion: let us assume that $a_{i}$ and $a_{j}$ are indifferent $\left(a_{i} I a_{j}\right)$. Weakening $a_{i}$ and reinforcing $a_{j}$ on one criterion (different or the same) lead to $a_{i}(P \cup I) a_{j}$. This condition ensures some coherence between the criteria and the global preferences.

- non redundancy: the family of criteria $\xi=\left\{\xi_{1}, \xi_{2}, \ldots, \xi_{q}\right\}$ does not contain any redundant criteria in the sense that the family obtained by removing any single criterion from $\xi$ would violate at least one of the two previous properties.

These three properties put together define a cosistent family of criteria. We refer the interested reader to (99) for formal definitions.

Example 5 The cafeteria problem.

Let us illustrate the condition of exhaustivity and cohesion in the context of the cafeteria problem:

- Two sellers $s_{i}$ and $s_{j}$ propose bids that are identical with respect to all the three criteria: $b_{i}=b_{j}=(685.000 C 1)$. Nevertheless, the manager states $b_{i} P b_{j}$. This statement is due to the fact that the manager has already had a successful contract with supplier $s_{i}$ in the past. Therefore, 
he's more likely to trust $s_{i}$ than an unknown supplier $s_{j}$. In order to correctly represent the preferences of the manager, one should consider an additional criteria related to the supplier's quality (this will be referred in chapter 3 to a bidder attribute). This example illustrates a case where the exhaustivity condition is not respected.

- Let us now assume that, instead of a fixed price for a yearly contract of 500 meals a day, the manager asks a daily price for one meal (which is, now, $\left.\xi^{1}\right)$. At the end of the year, the total price is calculated on the basis of the number of meals that have really been sold. Supplier $s_{i}$ proposes $b_{i}=\left(\begin{array}{l}8 \\ B\end{array} 3\right)$; the manager estimates that, if 3 different meals of quality $B$ are proposed to the employees, approximately $60 \%$ of them will frequent the cafeteria. As a consequence, the estimated yearly cost for the company is equal to 487.000 Euros $(=(8-1,5) * 500 * 250 * 0.6)$. Supplier $s_{j}$ proposes $b_{j}=(8 A 2)$. With this bid, the expectation of the manager about the employees' frequentation is the same. Therefore, he states that $b_{i} I b_{j}$. Now a third supplier $s_{k}$ proposes $b_{k}=\left(\begin{array}{ll}8 & A\end{array}\right)$. In this case, the employees' frequentation is expected to be equal to $80 \%$. Therefore, the estimated yearly cost raises to 650.000 Euros and thus, the manager states $b_{i} P b_{k}$ even if criterion $\xi^{3}$ has been reinforced. This example illustrates a case where the cohesion condition is not respected.

\subsubsection{The multicriteria decision aid problems}

Now that the basic multicriteria terminology and notions have been introduced we are ready to define what is a multicriteria decision problem.

Definition 8 (123) A multicriteria decision problem can be defined as a situation where given a set of actions $\mathcal{A}$ and a consistent family of criteria $F$ over $\mathcal{A}$, we want to solve one of the following problems:

- determine a subset of actions considered as the best considering $F$ (choice problem - $\alpha$ ),

- partition $\mathcal{A}$ in subsets with respect to pre-established norms (sorting problem - $\beta$ ), or

- rank order the set of actions $\mathcal{A}$ from best to worst (ranking problem $\gamma)$. 
Of course, a lot of real problems involve a mixture of these three main issues. Moreover, additional considerations may be cited:

- The description problem: help to describe actions and their consequences in a formalized and systematic manner to develop a cognitive procedure (99).

- Choosing $\mathbf{k}$ among $\mathbf{m}$ actions (8). This problematic can be viewed as a mixed of choice and ranking problematics.

- The design problem: to search for, identify or create new decision alternatives to meet the goals and aspirations revealed through the MCDA process (10).

- The porfolio problem: to choose a subset of alternatives from a larger set of possibilities, taking into account not only of the characteristics of the individual alternatives, but also of the manner in which they interact and their positive and negatives synergies (10).

Example 6 The cafeteria problem.

In this context, the manager is confronted with a suppliers' selection problem, i.e. a choice problematic. Let us note that, at the end of the negotiation, the manager has to select a unique supplier. Nevertheless, due to the diversity of offers, the auction might end with a set of good offers (and not necessarily a unique supplier). Consequently, the manager will have, for instance, to conduct one-to-one negotiations with the winning suppliers in order to select the unique winner. In this case, the auction is viewed as a first competitive step of the negotiation process (see also section 3.2).

\subsection{Methods}

The multicriteria problematic has inspired many authors and, therefore has benefited from an important number of theoretical contributions. Among the methods that are commonly used within the MCDA community, we will briefly introduce, here below, two major trends: the multiple attribute utility theory and the outranking methods.

This limitation is, of course, related to the minimal needs for a good understanding of the developments presented in future chapters. Of course, other approaches such as, for instance, interactive methods (77) or the analytical hierarchical process (AHP) (101) may deserve greater attention. 


\subsubsection{Multiple attribute utility theory}

Multi-Attribute Utility Theory (MAUT) $(48 ; 62)$ is an extension of the well-established utility theory to the multicriteria framework. In what follows, we will restrict ourselves to the context of certainty (even if this only constitutes a sub-part of MAUT). In this context, let us note that some authors (47) prefer to use the term value function instead of utility function.

The underlying idea of MAUT is that the decision maker's preferences can be represented by some function $U=U\left(\xi^{1}, \xi^{2}, \ldots, \xi^{q}\right)$ that aggregates all the criteria into values expressed on an artificial scale: the utility $(123 ; 20)$. Researchers working in the field of MAUT have been mainly focused on two distinct problems:

- what are the conditions under which the preferences of a decision maker can be represented by a specific utility function ?

- how to assess this function (and its related parameters)?

Of course, different explicit forms of $U$ have been proposed and studied in the literature. Among them, the most popular one is referred as the the additive model:

$$
U(a)=\sum_{k=1}^{q} U_{k}\left(\xi^{k}(a)\right)
$$

where the marginal utility ${ }^{5}, U_{k}$, is a non increasing function of $\xi^{k}$. Let us note that the weighted $\operatorname{sum} U(a)=\sum_{k=1}^{q} \omega_{k} \cdot \xi^{k}(a)$ is a particular case of the additive model.

In order to apply an additive model a necessary condition is the preference independence assumption. Intuitively, two criteria are said to be preferentially independent if the trade-offs between them are independent of all the other attributes (47). More formally:

Definition 9 (47) Let $I \subset\{1, \ldots, q\}$ be a subset of attribute indices and let $X_{I}$ represent the subset of the attributes designated by the subscripts in $I$. Let $\bar{X}_{I}$ be the complementary subset of the $q$ attributes. Then,

\footnotetext{
${ }^{5}$ The use of marginal utility functions permits to transform the values of the different criteria $\xi^{k}$ into a common abstract scale. Therefore, problems related to different units are avoided and the sum of every marginal contributions is meaningful.
} 
- $X_{I}$ is preference independent of $\bar{X}_{I}$ if $\left(w_{I}, \bar{w}_{I}\right) \mathcal{S}\left(x_{i}, \bar{w}_{I}\right)$ for any $w_{I}, x_{I} \in$ $X_{I}$ and $\bar{w}_{I} \in \bar{X}_{I}$ implies $\left(w_{I}, \bar{x}_{I}\right) \mathcal{S}\left(x_{I}, \bar{x}_{I}\right)$ for all $\bar{x}_{I} \in \bar{X}_{I}$.

- The attributes $X_{1}, \ldots, X_{q}$ are mutually preference independent if for every subset $I \subseteq\{1, \ldots, q\}$ the set $X_{I}$ of these attributes is preference independent of $\bar{X}_{I}$.

The following example illustrates a case where the preference independence assumption is violated.

Example 7 The cafeteria problem

In order to illustrate a situation of preferential dependence, let us consider the data listed in table 2.2. The manager will state that $b_{2} P b_{1}$ since, for a meal of quality $B$, he prefers to pay 150.000 Euros more to be able to offer more variety to the employees. On the contrary, when comparing $b_{3}$ and $b_{4}$ he will state $b_{3} \mathrm{~Pb}_{4}$ : since for a meal of quality $A$, he considers that a variety equal to 2 meals a day is already sufficient and, therefore he prefers to save 150.000 Euros. Nevertheless, both $b_{1}$ and $b_{3}$, and $b_{2}$ and $b_{4}$ have the same evaluation according to the variety and price criteria - only the quality varies. As a consequence, the criterion $\{$ quality\} is not preferentially independent to the criteria $\{$ variety,price $\}$.

Table 2.2: Example of 4 bids leading to a preferential dependence situation.

\begin{tabular}{|c|ccc|}
\hline Bids & Variety & Quality & Price \\
\hline$b_{1}$ & 2 & $\mathrm{~B}$ & 1.000 .000 \\
$b_{2}$ & 3 & $\mathrm{~B}$ & 1.150 .000 \\
$b_{3}$ & 2 & $\mathrm{~A}$ & 1.000 .000 \\
$b_{4}$ & 3 & $\mathrm{~A}$ & 1.150 .000 \\
\hline
\end{tabular}

Of course, even if the decision maker is assumed to react according to the additive model (and consequently his preferences are assumed to be compatible with this model), the problem is only half-solved since the marginal utility functions still have to be determined. To perform this task, different approaches have been proposed. Among them, we can cite:

\section{- Direct methods}

Direct methods permit to evaluate the parameters characterizing the 
multi-attribute utility function by questioning the decision maker about his preferences. Of course, different questioning procedures exist (123). For the illustrative purpose of this section, let us outline the Pricing Out Technique (62). To apply this technique, at least one price attribute must be involved in the decision problem. Let us assume that $\xi^{1}$ is the price and $\xi^{2}, \ldots, \xi^{q}$ are non-priced attributes. The aim of this technique is to price out all the non price attributes, i.e. to transform their values into monetary values. Once this step has been done, all the criteria can be aggregated into a monetary scale (in other words, in this case, utility is money). To assess the monetary value of the different attributes values, one presents to the decision maker two actions:

$$
\begin{aligned}
& -x=\left(p_{x}, x^{1}, x^{2}, \ldots, x^{q-1}\right) \\
& -\tilde{x}=\left(?, \tilde{x}^{1}, \tilde{x}^{2}, \ldots, \tilde{x}^{q-1}\right.
\end{aligned}
$$

Then, one asks the decision maker the price of $\tilde{x}$ such that he would be indifferent between $x$ and $\tilde{x}$. If he answers $p_{\tilde{x}}$, this is interpreted as follows: the decision maker is ready to pay $p_{\tilde{x}}-p_{x}$ to transform the bundle of non price attributes of $x$ into the bundle non price attributes of $\tilde{x}$.

\section{- Indirect methods}

With indirect methods, the analyst tries to assess the multi-attribute utility function from global judgements expressed by the decision maker on $\mathcal{A}$. For instance, the decision maker gives a ranking of the alternatives belonging to $\mathcal{A}$. Then the analyst tries to fit the parameters of a specific multi-attribute function in order to respect this ranking. An example of such a method is the UTA method (56). In this case, a linear program is used to find the most accurate parameters' fitting.

Example 8 The cafeteria problem.

The manager decides to base the bids'selection on the following multi-attribute linear utility function:

$$
U(b)=\omega_{1} \cdot U^{1}\left(b^{1}\right)+\omega_{2} \cdot U^{2}\left(b^{2}\right)+\omega_{3} \cdot U^{3}\left(b^{3}\right)
$$

where the marginal utility functions are defined as follows:

- $U^{1}\left(b^{1}\right)=1-\left(b^{1}-187.500\right) / 1.375 .000$. It has been decided that the employees will pay their daily lunch 1,5 Euros: the company supplies the 
difference between the meal price and this fixed price. Therefore, the company's contribution is equal to the yearly price minus the employees'contribution $(187.500=1,5 * 500 * 250)$. Finally, since the reserve price for a daily meal has been fixed to 12, 5 Euros, the maximum contribution of the company is equal to $1.375 .000(=12,5 \cdot 250 \cdot 500-187.500)$ which is used as a normalization factor.

- $U^{2}(1)=0, U^{2}(2)=0.5, U^{3}(3)=1$

- $U^{3}(C)=0, U^{3}(B)=0.5, U^{3}(A)=1$

After a detailed analysis of the buyer's preferences, the weights $\left(\omega_{1} \omega_{2} \omega_{3}\right)$ have been fixed to $\left(0,6544733490,143981769\right.$ 0,201544881) ${ }^{6}$. Consequently, the utility assigned to the first offer $b(1)=(685.000 C 1)$ is equal to:

$U[b(1)]=0,6544 \cdot\left[1-\frac{685.000-187.500}{1.375 .000}\right]+0,1439 \cdot 0+0,2015 \cdot 0=0,418$

In many multi-attribute auctions, the buyer is not willing to communicate his utility function to the sellers (since this information is often private). On the other hand, suppliers need some information to know how to improve their bids. One solution is to provide them a bidding support system: the seller propose a bid the components of which are all determined except the $j^{\text {th }}$ one. Then, the system (based on the buyer's utility function) determine the maximal value of this attribute (if the criterion has to be minimized) such that the resulting bid satisfies the auction rule (this approach will be further described in section 5.4.3).

To illustrate this, let us consider that another supplier decides to propose a new offer. In order to be accepted, this bid must satisfy the auction rule, i.e. its utility must be at least $0,05^{7}$ higher than the current best utility (0.418). The buyer uses the bidding support system in order to determine the components of different potential bids. Therefore, he considers different configurations for quality and variety attributes and let the system determine the minimal price in order to reach a 0.423 utility score. The results are presented in table 2.3.

\footnotetext{
${ }^{6} \mathrm{~A}$ major critic about multi-attribute linear utility functions concerns the difficulty to correctly interpret the weights. The present example perfectly illustrates this assertion

${ }^{7}$ This parameter is fixed before the auction.
} 
Table 2.3: Bidding support system. Different bid configurations. Utility scores

\begin{tabular}{|ccc|c|}
\hline Variety & Quality & Determined price & Utility Score \\
\hline 3 & 3 & 1.400 .000 & 0.423 \\
2 & 2 & 1.037 .500 & 0.423 \\
2 & 1 & 825.000 & 0.423 \\
\hline
\end{tabular}

\subsubsection{Outranking methods}

The objective information that can be extracted from a multicriteria decision problem is relatively poor: the dominance relation can help the decision maker to detect (and eliminate) dominated actions but, in most cases, an important number of efficient alternatives remain. On the contrary, the results obtained using a multi-attribute utility approach are excessively rich since every pair of actions are comparable. Nevertheless, this complete pre-order ${ }^{8}$ is obtained at the expense of restrictive assumptions and, sometimes, undue subjective information asked to the decision maker (20).

A number of arguments plead in favor of methods that are based on more flexible assumptions. Indeed, the quest for complete pre-orders is not always justified (122):

- since, in some cases, partial pre-orders do not put a brake on the decision process. For instance, if $a P b, a P c$ and $b J c$, one does not have to force the comparison between $b$ and $c$ to conclude that $a$ is the best alternative:

- since the existence of potential incomparability between two actions may highlight singularities of the decision problem:

- since, due to the dynamic of the decision process, holding some incomparability between alternatives might be meaningful.

In reaction to these arguments, outranking methods have been developed: they can be intuitively viewed as compromises between the poor objective dominance relation and the rich subjective multi-attribute utility functions. More formally:

\footnotetext{
${ }^{8}$ a complete pre-order is a binary relation $R$ that is complete (i.e. $a R b$ or $b R a$ $\forall a, b \in \mathcal{A})$, reflexive and transitive. If the relation is not complete, it is referred to as a partial pre-order.
} 
Definition 10 (99) An outranking relation is a binary relation $\mathcal{S}$ defined in $\mathcal{A}$ such that $a_{i} \mathcal{S} a_{j}$ if, given what is known about the decision maker's preferences and given the quality of the valuations of the actions and the nature of the problem, there are enough arguments to decide that $a_{i}$ is at least as good as $a_{j}$, while there is no essential reason to refuse that statement.

In order to illustrate this definition, we will outline, in the next subsections, two outranking methods: the Electre I and Promethee I and II methods.

\subsubsection{The Electre I method}

The Electre $I^{9}$ (95) method has been designed to manage multicriteria choice problems ( $\alpha$ problematic). As shown below, the building of the $\mathcal{S}$ relation perfectly illustrates the definition of an outranking relation.

In what follows, we will assume that a weight $\omega_{j}$ has been assigned to each criterion $\xi_{j}\left(\omega_{j} \geq 0, \sum_{j=1}^{q} \omega_{j}=1\right)$.

When comparing two actions $a_{i}$ and $a_{j}$, two indexes will be computed: a concordance index and a discordance index. The concordance index quantifies the fact that $a_{i}$ is at least as good as $a_{j}$ : it is "percentage" of the weights related to criteria such that $\xi^{k}\left(a_{i}\right) \leq \xi^{k}\left(a_{j}\right)$. More formally:

$$
C\left(a_{i}, a_{j}\right)=\sum_{k \mid \xi^{k}\left(a_{i}\right) \leq \xi^{k}\left(a_{j}\right)} \omega_{k}
$$

On the other hand, the discordance index quantifies if there is an essential reason to refuse to state that $a_{i}$ is at least as good as $a_{j}$. In other words, if $a_{j}$ is much better than $a_{i}$ on at least one criterion. More formally:

$$
d\left(a_{i}, a_{j}\right)=\frac{1}{\delta} \max _{k}\left\{\xi^{k}\left(a_{i}\right)-\xi^{k}\left(a_{j}\right), 0\right\}
$$

where $\delta=\max _{a_{i}, a_{j}, k}\left\{\xi^{k}\left(a_{i}\right)-\xi^{k}\left(a_{j}\right)\right\}$. Intuitively, if $d\left(a_{i}, a_{j}\right)=0 \Leftrightarrow$ $\xi^{k}\left(a_{i}\right) \leq \xi^{k}\left(a_{j}\right), \forall k$ and thus there is no reason to refuse to state that $a_{i}$ is at least as good as $a_{j}$. Whenever, $\exists k \mid \xi^{k}\left(a_{j}\right) \leq \xi^{k}\left(a_{i}\right)$ the discordance index becomes positive. Once this index exceeds a level $\tilde{d}$ defined by the decision

\footnotetext{
${ }^{9}$ ELECTRE is the acronym for ELemination Et Choix Traduisant la REalité
} 
maker, one may assume that there is enough reason to state that $a_{i}$ is not at least good as $a_{j}$.

The combination of these two indexes permits to define the outranking relation $\mathcal{S}$ :

$$
a_{i} \mathcal{S} a_{j} \Leftrightarrow C\left(a_{i}, a_{j}\right) \geq \tilde{c}, d(a, b) \leq \tilde{d}
$$

where the parameters $\tilde{c}$ and $\tilde{d}$ are fixed by the decision maker. Let us note that, since the first version of Electre I, many variations have been proposed to formalize the concept of discordance. Among others, most of the authors consider a discordance parameters $\tilde{d}_{k}$ for each criterion. As a consequence, the previous rule becomes:

$$
a_{i} \mathcal{S} a_{j} \Leftrightarrow C\left(a_{i}, a_{j}\right) \geq \tilde{c}, \xi^{k}(a)-\xi^{k}(b) \leq \tilde{d}_{k}, \forall k
$$

Once the outranking relation $\mathcal{S}$ has been setup, one need to exploit it in the choice context. Therefore, the notion of kernel has been proposed.

Definition 11 (123) The kernel $N$ is a subset of the set of actions $\mathcal{A}$ such that $\forall a_{j} \in \mathcal{A} \backslash N, \exists a_{i} \in N: a_{i} \mathcal{S} a_{j}$ and $\forall a_{i}, a_{j} \in N: a_{i} \neg \mathcal{S} a_{j}$

The kernel represents the subset of actions considered to be the best with respect to $\mathcal{A}$ and $\xi$. Indeed, every action outside the kernel is outranked by at least one action of the kernel and actions within the kernel are incomparable. Let us note that, as a direct corollary, some actions belonging to the kernel could be outranked by actions outside the kernel. To illustrate this purpose, let us consider the following case: $b \mathcal{S} c, b \mathcal{S} a$ and $c \mathcal{S} d$. In this case, $N=\{b, d\}$ while $c \mathcal{S} d$. Additionally, let us stress that the kernel is not always unique (which is the case when the outranking relation contains circuits).

The Electre I dates back from 1968 (95). Since this first publication, a number of evolutions have been made. Among others:

- Electre II (96) is an extension of the Electre I developed for ranking problems (problematic $\gamma$ );

- Electre III (97) is an extension of Electre II based on pseudo-criteria (123); 
- finally Electre TRI (130) has been designed to manage sorting problems.

We refer the interested reader to $(99 ; 123 ; 47 ; 74)$ for a detailed analysis of the Electre methodology. Finally, let us stress that a detailed example illustrating the Electre I will be presented in chapter 4 .

\subsubsection{The Promethee methods}

The Promethee ${ }^{10}$ methods $(19 ; 22 ; 23)$ have been developped in order to manage ranking problematics. In this subsection, we sketch an outline of this methodology.

Just as the Electre methods, the Promethee methods are based on pair wise comparisons. When comparing two actions $a_{i}$ and $a_{j}$ on criterion $\xi^{k}$ the difference of evaluations between these two actions should be taken into account. Assuming that $\xi^{k}\left(a_{i}\right) \leq \xi^{k}\left(a_{j}\right)$ this difference can be stated as follows,

$$
d_{k}\left(a_{i}, a_{j}\right)=\xi^{k}\left(a_{j}\right)-\xi^{k}\left(a_{i}\right)
$$

When the difference $d_{k}\left(a_{i}, a_{j}\right)$ is small and the DM can neglect it, there is no reason to say that $a_{i}$ is preferred to $a_{j}$ and consequently the actions are indifferent (for the specific criteria $\xi^{k}$ ). The higher the value of $d_{k}$, the larger the preference $P_{k}\left(a_{i}, a_{j}\right)$ in favor of $a_{i}$ over $a_{j}$, on criterion $\xi^{k}$. This preference can be defined through a preference function in the following way,

$$
P_{k}\left(a_{i}, a_{j}\right)=f_{k}\left(d_{k}\left(a_{i}, a_{j}\right)\right), \quad \forall a_{i}, a_{j} \in \mathcal{A}
$$

and we can assume that $P_{k}\left(a_{i}, a_{j}\right) \in[0,1]\left(\right.$ if $P_{k}\left(a_{i}, a_{j}\right)>0$, then $P_{k}\left(a_{j}, a_{i}\right)=$ $0)$.

The pair $\left(\xi^{k}, P_{k}\left(a_{i}, a_{j}\right)\right)$ is called a generalized criterion associated with criterion $\xi^{k}$, for all $k \in\{1, \ldots, q\}$. Only 6 types of generalized criteria are considered in the Decision Lab software (125). Generalized criterion of type 5 requires the definition of both $q_{k}$ and $p_{k}$ (see Figure 1 ).

\footnotetext{
${ }^{10}$ PROMETHEE is the acronym of Preference Ranking Organisation METHod for Enrichment Evaluations
} 


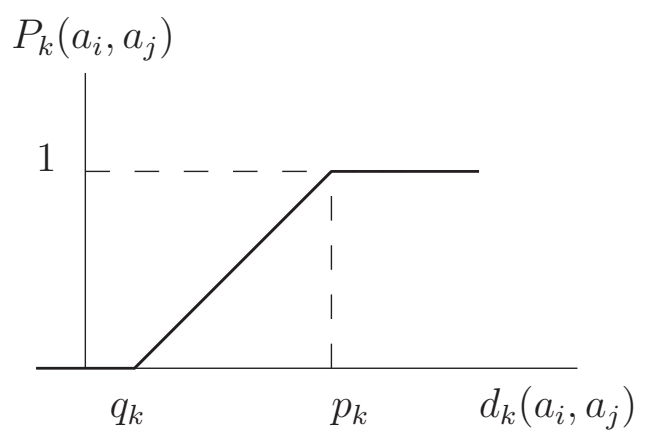

Figure 2.1: Generalized criterion of type 5

Once the preference degrees between two actions $a_{i}$ and $a_{j}$ have been computed for every criterion, one need to aggregate these marginal contribution to obtain $P\left(a_{i}, a_{j}\right)$ i.e. a global measure of the preference of $a_{i}$ on $a_{j}$. This is performed using a classic weighted sum $\left(\omega_{k}\right.$ is assumed to be the weight associated to criterion $\xi^{k}$ ):

$$
P\left(a_{i}, a_{j}\right)=\sum_{k=1}^{q} \omega_{k} \cdot P_{k}\left(a_{i}, a_{j}\right)
$$

The fundamental idea underlying the PROMETHEE methods is the quantification of how an action a outranks all the remaining $(p-1)$ actions and how $a$ is outranked by the other $(p-1)$ actions. This idea leads to the definition of the positive $\phi^{+}(a)$ and negative $\phi^{-}(a)$ outranking flows. More formally:

$$
\begin{aligned}
\phi^{+}\left(a_{i}\right) & =\frac{1}{p-1} \sum_{a_{j} \in \mathcal{A}, i \neq j} P\left(a_{i}, a_{j}\right) \\
\phi^{-}\left(a_{i}\right) & =\frac{1}{p-1} \sum_{a_{j} \in \mathcal{A}, i \neq j} P\left(a_{j}, a_{i}\right)
\end{aligned}
$$

Given these two measures, two total pre-order of $\mathcal{A}$ can be obtained (one associated to the values of $\phi^{+}$and another associated to the values of $\phi^{-}$). The intersection of these two pre-order lead to a partial pre-order called 
the Promethee I ranking. In this context, two actions $a_{i}$ and $a_{j}$ will be judged to be incomparable if $\phi^{+}\left(a_{i}\right)>\phi^{+}\left(a_{j}\right)$ and $\phi^{-}\left(a_{i}\right)>\phi^{-}\left(a_{j}\right)$ or of $\phi^{+}\left(a_{i}\right)<\phi^{+}\left(a_{j}\right)$ and $\phi^{-}\left(a_{i}\right)<\phi^{-}\left(a_{j}\right)$.

On the other hand, the complete pre-order obtained with the Promethee II method is based on the net flow $\phi\left(a_{i}\right)$ assigned to each a action $a_{i} \in \mathcal{A}$.

$$
\phi\left(a_{i}\right)=\phi^{+}\left(a_{i}\right)-\phi^{-}\left(a_{j}\right)
$$

Let us note that,

$$
\phi\left(a_{i}\right)=\frac{1}{n-1} \sum_{k=1}^{q} \sum_{a \in \mathcal{A}}\left\{P_{k}\left(a_{i}, a\right)-P_{k}\left(a, a_{i}\right)\right\} \cdot \omega_{k}=\sum_{k=1}^{q} \phi_{k}\left(a_{i}\right) \cdot \omega_{k}
$$

where $\phi_{k}\left(a_{i}\right)$ is called the $k^{\text {th }}$ unicriterion net flow assigned to action $a_{i}$. Intuitively, these values allow to better locate action $a_{i}$, according to criteria $\xi^{k}$, with respect to all the other actions in $\mathcal{A}$.

In addition to these rankings, Mareschal and Brans (71) have proposed a geometrical tool that helps the decision maker both to interactively explore and structure the decision problem and to better understand the results provided by the Promethee rankings. This is referred to as the Gaia plane. The underlying idea of this approach is to perform a principal components analysis on the unicriterion net flows assigned to each action (23).

A number of applications have been treated using the Promethee methods (see (23) for detailed references). Both the Promethee I and II rankings and the Gaia plane are implemented in Decision Lab (125). Moreover, the software offers interesting functionalities regarding the exploration and structuring of the problem, the sensitivity analysis of the results or the management of group decision problems.

Of course, since the first contributions, the Promethee methodology has known a number of improvements. Among others, let us mention:

- Promethee III (23): an extension of Promethee II that is based on a more careful definition of indifference and preference relations (the net flow is enriched by a standard deviation).

- Promethee V (21): an extension to Promethee I and II to address the Portfolio problematic. 
- Promethee TRI and Promethee CLUSTER (46). The aim of this work is to extend the Promethee method to multicriteria classification problems: sorting and clustering problems (see also (33)).

Finally, let us stress that critics on the use of the Promethee method can be found in (32). Among them, the most commonly cited one is related to the third alternative dependence (the relative ranking of two alternatives can be swapped due to the adding or deleting of a third alternative ${ }^{11}$. Let us note that this effect is also present in other methods.

Example 9 The cafeteria problem.

Facing the 3 potential bid configurations listed in table 2.3, supplier $s_{i}$ decides to use the Promethee method to select the bid he will propose.

First of all, he decides to compute the margin he will realize for each bid configuration (see table 2.4). The (daily) cost related to one meal characterized by a quality $Q$ and a variety $V$ is equal to $5+1,5 \cdot(Q-1)+(V-1)$.

Table 2.4: Bidding support system. Different bid configurations. Margin for supplier $s_{i}$.

\begin{tabular}{|ccc|c|}
\hline Variety & Quality & Price & Margin for supplier $s_{i}$ \\
\hline 3 & 3 & 1.400 .000 & 150.000 \\
2 & 2 & 1.037 .500 & 100.000 \\
2 & 1 & 825.000 & 75.000 \\
\hline
\end{tabular}

Naturally, $s_{i}$ wants to maximize his margin. On the other hand, in order to limit the risk associated to the stock management, he prefers to minimize the meals'variety. Finally, his marketing strategy (i.e. his public image) leads him to maximize the meal quality. Figure 2.2 summarizes his preferences:

- the realized margin is as important as the two non-price attributes. An difference lower than 5.000 Euros is to small to be taken into account. On the other hand, as soon as it exceeds 25.000 Euros, a strict preference is set. Between these two values, a linear interpolation is done.

- concerning the variety attribute, a difference of 1 unit is sufficient to have a strict preference

\footnotetext{
${ }^{11}$ even if this one is dominated by all the other actions
} 
- on the contrary, the quality attribute is interpreted using a level preference function. A difference of two levels, lead to a strict preference (i.e. the preference degree is equal to 1), while a difference of 1 level is judged to be too small to be quantified (i.e. the preference degree is equal to 0).

These settings lead to a partial ranking with two interesting bids: $b_{1}$ and $b_{2}$. Indeed, $b_{3}$ is outranked since it has the lower margin and the lower quality (see figure 2.2).

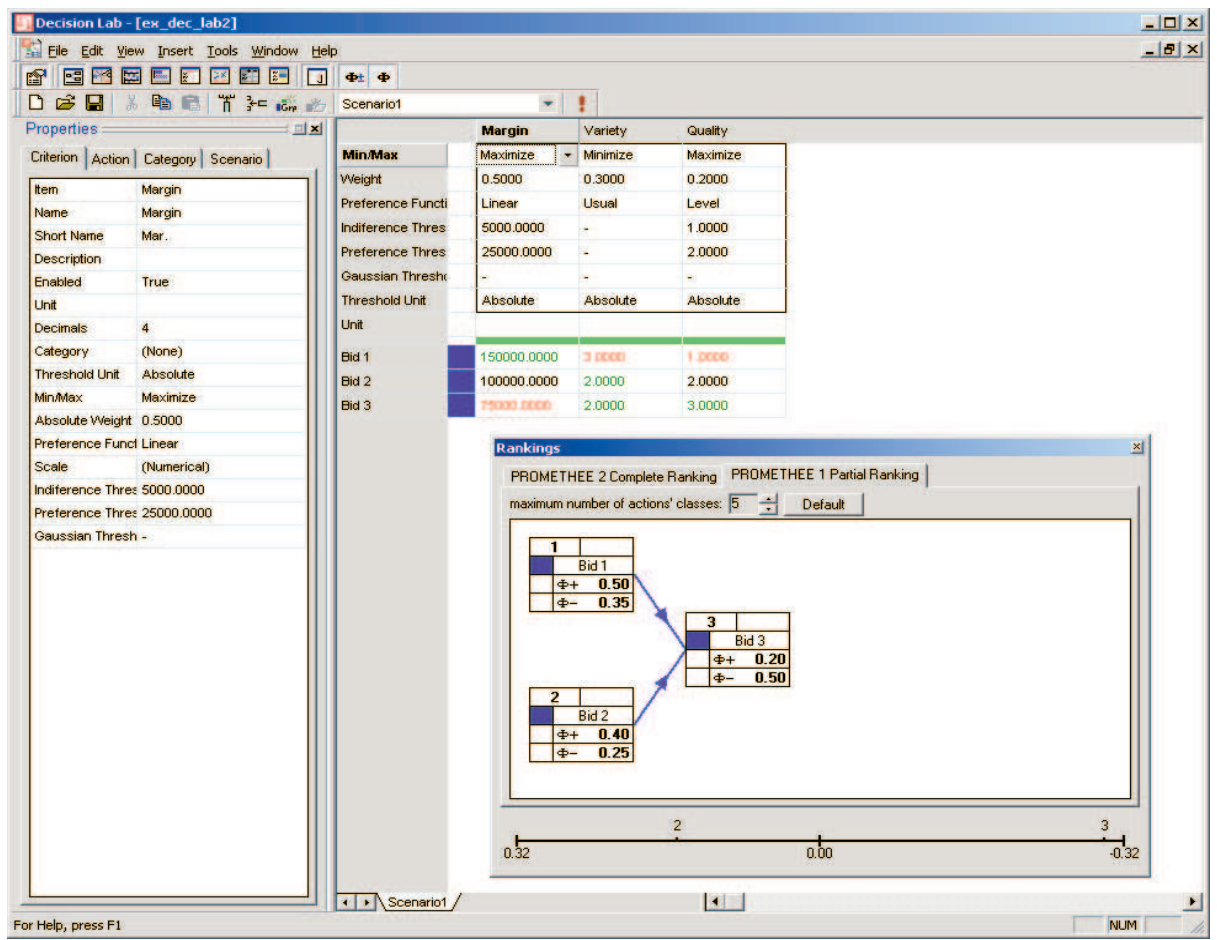

Figure 2.2: Promethee I partial ranking

In order to illustrate the fact that the Promethee rankings are affected by the number of actions, let us, temporarily, focus on bids $b_{1}$ and $b_{2}$ (we do not consider anymore $b_{3}$ in the analysis). As shown on figure 2.3, $b_{1}$ appears to be the best bid while these two bids were incomparable before (let us note that this result is, however, compatible with the first Promethee II ranking obtained). 


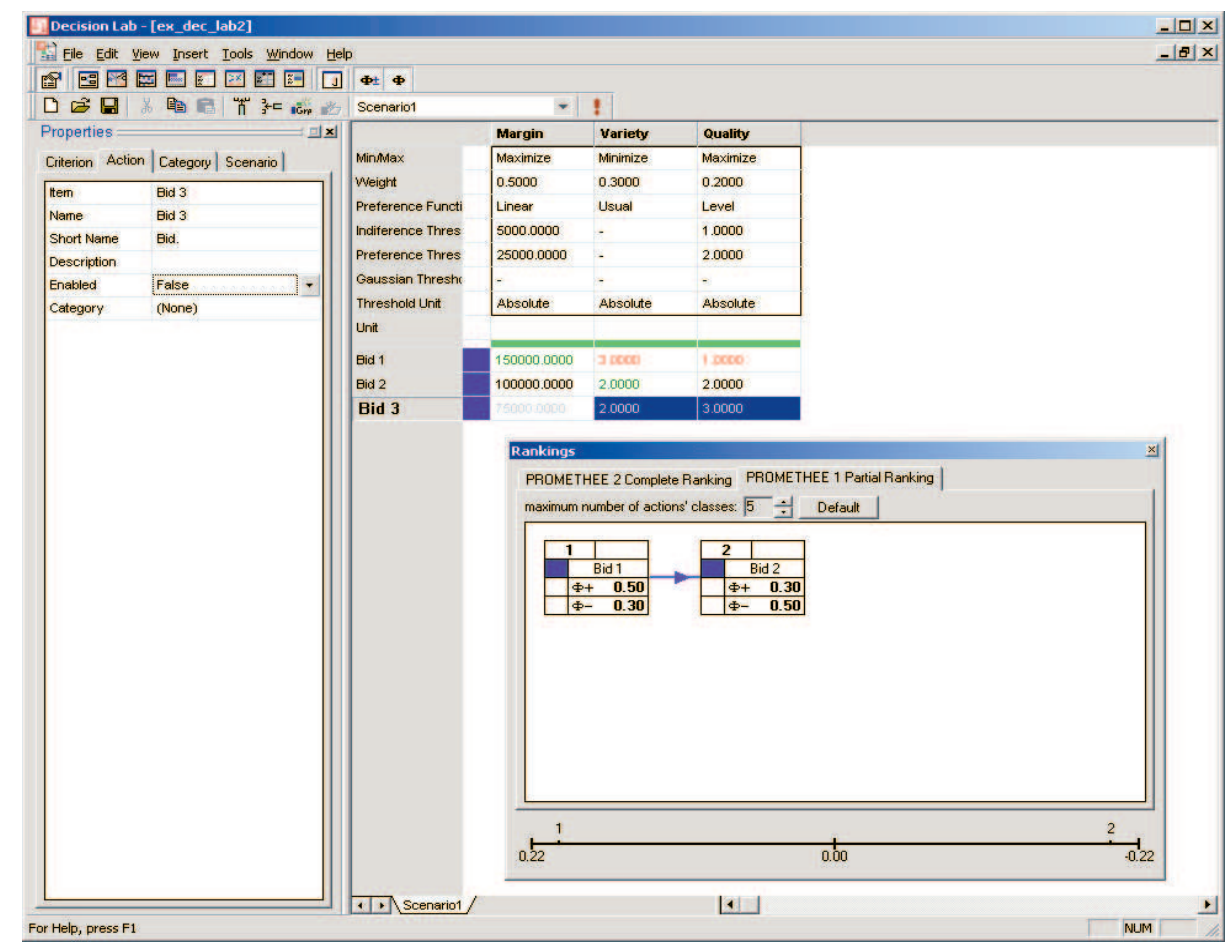

Figure 2.3: Promethee I partial ranking, when bid $b_{3}$ is not considered anymore.

\subsubsection{A few selected modeling issues}

Of course, when applying multicriteria methods, many modeling issues have to be considered. Among them, we will sketch a few of them that seem to us to be particularly relevant:

\section{- Criterion's scale}

As already stressed, a criterion is, basically, an application from the set of actions $\mathcal{A}$ into an ordered set $E$. Nevertheless, the elements of $E$ can be belong to different scales (100):

- ordinal scale: the gap between two degrees does not have a clear meaning in terms of preference differences.

- ratio scale: a numerical scale whose degrees are defined by reference to a clearly defined unit in a way that gives meaning, on the one hand, to the absence of quantity (degree 0) and, on the other hand, to the ratio of the numbers which characterize them, 
each of them being interpretable as the addition of a given number (integer of fractional) of units of the quantity considered.

- interval scale: the ratio of numbers which characterize two degrees are not significant while the ratio between differences in numbers associated with two pairs of distinct degrees is significant.

Example 10 With reference to the illustration of the Promethee method (see figure 2.2), the \{margin\} has been associated to a ratio scale, the \{variety\} has been associated to an ordinal scale while the $\{$ quality $\}$ has been associated to an interval scale.

Different scales mean different things, even if the information coding is the same. Therefore, this distinctive feature has to be carefully managed during the modeling activity (to avoid, for instance, the abusive aggregation of heterogeneous scales into a ratio scale).

\section{- Inter-criteria information}

An intrinsic problem related to multicriteria decision aid is to be able to identify and, eventually, to quantify the relative importance between the criteria.

In this context, the weakest information needed is a ranking of the criteria. This ordinal information is, for instance, exploited in the lexicographic method and in Argus (31). If one looks to quantify this relative importance, the notion of weight appears.

\section{- Weights}

The term weight sounds familiar and basic. Nevertheless, it hides a wide variety of distinct interpretations. Among them, let us cite (27):

* marginal contributions per unit of $\xi^{j}$;

* indifference trade-offs or rates of substitution;

* gradient of an overal value function;

* discriminating power of the criteria on the alternatives;

* parameters used in interactive optimization;

* scaling factors converting into commensurate overall value;

$* \ldots$

For instance, in the classic weighted sum, the weights have to be interpreted as scaling factors converting the evaluations into a commensurate overall value and, as a consequence, naturally 
depend of the criterion's scale (if the scale changes, the weight has to be changed (122)). Therefore, they cannot be interpreted as the relative importance of the criteria (unless all the criteria already belong to the same scale). On the other hand, the weights used in the Electre I method (which is not compensatory) have to be interpreted as the number of votes in a voting procedure. These two examples illustrate perfectly the fact that the notion of weights has to be carefully managed when using a specific model.

\section{- Preferential dependence}

As already stressed in section 2.3.1, preferential independence is a necessary condition to be able to model the decision maker's preferences using a multi-attribute linear utility function (or associated approaches). However, a number of situations do not satisfy this assumption.

Recent works $(80 ; 50 ; 72)$ nevertheless offer an elegant solution to this problem. They rely on the use of the choquet integral as aggregation operator. In these works, the notion of weights per criterion are extended to weights for coalitions of criteria. As a consequence of the sub or sur-additivity of these weights, synergies and redundancies between set of criteria can be taken into account in the model.

\section{- Parameters fitting}

As already stressed, a number of parameters are involved in multicriteria methods: inter-criteria parameters, such as weights or discordance thresholds, or intra-criteria parameters, such as indifference of preference thresholds. Of course, the fitting of these parameters is a crucial step in the modeling process. Moreover, their clear interpretation by the decision maker remains another difficulty.

\subsection{Conclusion}

This chapter constitutes a rough introduction to multicriteria decision aid. Our aim was to introduce the basic vocabulary and notions that will be used in the next chapters. Moreover, the Promethee I and II and Electre I methods have been outlined. These approaches have inspired us to develop the butterfly model presented in chapter 5 .

Of course, Multicriteria Decision Aid cannot be (naively) reduced to the 
application of a given mathematical method to an idealized problem. It is, first of all, a complex activity where the problem structuring is vital. This aspect has nevertheless been under represented in the previous pages. We invite the interested reader to consult the book written by Bernard Roy (99) for such a discussion and the recent state of the art surveys (47) for a detailed analysis of the field. 


\section{Chapter 3}

\section{Multi-attribute auctions}

\subsection{Introduction}

Procurement is a key issue for any company and has a deep impact on the supply chain management. Therefore, e-Procurement solutions have received great attention and, are more and more used, replacing traditional practices. According to the Aberdeen Group (1), revenues related to e-Sourcing activities will exceed $\$ 3$ billion by 2005 .

One way to conduct e-Procurement rely on the use of reverse auctions. For instance, in 2000, General Motor, Ford and Daimler-Chrysler joined together to create an electronic market place based on auctions, called Covisint, for the procurement of basic elements (common plastic parts, bolts, steal,... ). Renault and Nissan joined the group some time later (nowadays Covisint has been integrated in Ariba (5)) . Another example is the WorldWide Retail Exchange (126) which is an electronic market place based, among others, on e-auctions for the sourcing in the retail and consumer goods industry. Business to Government (B2G) constitutes another application field for reverse auctions. For instance, the Office of Government Commerce (85) reports recent successful implementations in the U.K.

The use of traditional reverse auctions is particularly well adapted when the items (objects, services, ...) are commodity-like (i.e. more or less homogeneous). Stocks, electricity, bolts, metal constitute such examples. However, as stressed by Milgrom (79), ... price-only auctions are of little value if the goods, or their suppliers, have widely varying characteristics, because the buyer will not normally buy based on a comparison of prices alone. In the 
automobile example mentioned above, price-only auctions are not suited for the procurement of elements requiring detailed technical specifications or an important implication of the supplier in the design.

In practice, when items to be purchased are heterogenous, Request for Quotes $(\mathrm{RFQ})^{1}$ are used: the buyer specifies the minimal requirements for the item, lists a number of negotiable attributes and invite the suppliers to submit offers. Once the bids have been collected, the buyer ranks and selects the winning supplier. Of course, this task is time consuming and relatively tricky: offers may differ considerably among the suppliers (in terms of variety but also due to their (detailed) contents). As a consequence, most of the time, only a restricted number of suppliers are contacted in practice. Finally, such processes are often regularly conducted. An attempt to automated such an activity is to develop auctions mechanisms that support bids with several attributes.

Multi-attribute auctions are relatively new. Early works date from the beginning of the nineties and most of the contributions on the subject have less than five years old. At the same time, researchers, with different backgrounds, such as economy, computer science, mathematics, ... have developed models to solve the same problem but by using different terminologies. That's the reason why we now speak about multidimensional auctions $(25 ; 18 ; 118 ; 66)$, multi-attribute auctions $(12 ; 11 ; 112 ; 111)$, scoring auctions (6) or multiple issue auctions (114).

To our knowledge, the first contributions on the subject $(118 ; 25)$ have used the term multidimensional auctions. However this designation has been used in different contexts. Some authors (128) use it to refer to 'classic' multiobjects auctions while others use it to refer to auctions combining multi-unit, multi-objects and multi-attribute features (see section 3.7). To avoid any ambiguity we have decided to use the term multi-attribute auctions in the present work. Furthermore, this choice seems to be a common trend in the field.

As pointed out by Teich et al. (115), a further distinction must be made between the terms 'issues' and 'attributes'. Many researchers use the term attribute to refer to the characteristics of a bid such as, for instance, price, quality, terms of delivery, ... Teich et al. rather use the term Negotiable Bid Issues (NBI) while the term Bidder Attribute (BA) is related to the bidder himself: Is he ISO certified ? Has the auctioneer historical business

\footnotetext{
${ }^{1}$ in what follows, we will not distinguish Request for Quotes, Request for Bids or Request for Proposals
} 
relationship with the considered bidder ? ... This distinction is important, especially when the auctioneer wants to discriminate the bidders. In the present work, this distinctive feature is not the center of interest and the word attribute will be used.

Until now, we have exclusively motivated the use of multi-attribute auctions as a consequence of the intrinsic complexity and variety of the items traded (forcing the simultaneous consideration of several criteria). As stressed by Bichler (13), another cause lies in the fact that suppliers are often tempted to explicitly differentiate their products even if a priori they are relatively homogeneous (in order to avoid the fatal direct and easy comparisons). For instance, Airlines company offer discounts for frequent flyers, week-end tariffs, for early registration ... Therefore, automating the search for the best deal related to a trip between Brussels and New York next Monday is not easy.

A number of examples have been cited in the literature to motivate bids characterized by several attributes. Here are some of them:

- Stark (110) analyzes the context of highway building contracts. In such context, the buyer estimates the quantities related to the different elements needed for the building (number of tons of asphalt,... ). Then, each bidder provides a multidimensional bid characterizing the unit price for these elements. The winning supplier is the one who proposed the cheapest expected contract however he is paid according to the true quantities used.

- Cripps and Ireland (30) consider the context of UK television franchise; in addition to the price a quality level (incorporating likely share of viewers, live programs, ...) is considered

- Che (25) mention the case of Department of Defense procurements. Typically, in such situations, the non-price attributes are at least as important as the price itself.

- Bichler $(13 ; 12 ; 11 ; 14)$ uses the example of OTC derivatives trading to empirically test multi-attribute auctions. In another contribution he describes an electronic market place for the procurement of large food retailers. Applications for the procurement of computers and in the Tourism sector are also considered ...

- Teich et al. illustrate their system, NegotiAuction, for the procurement of non-defective chemical weapons protection suits. 
On the other hand, Teich et al. (115) cite a few web sites managing multi-attribute RFQ or reverse auctions. Among others, FreeMarkets, Ariba, Perfect, Ebreviate, CombineNet, ... As stressed by the authors, it is often difficult to know to which extend the technology incorporates multi-attribute tools (since this information is often disclosed).

A few authors have studied multi-attribute auctions from an economical perspective. The description of their works goes beyond the scope of this chapter and we refer the interested reader to Thiel (118), Che (25), Branco (18) and Asker et al. (6) for details.

This chapter is organized as follows. The next section is dedicated to preliminary observations. Then, in section 3.3, we will describe different ways that have been proposed to manage several attributes. Then, a common framework proposed by Teich et al. (115) will be presented in section 3.4 and will be completed in section 3.5 with the presentation of a few evaluation criteria. Experimental studies will be outlined in section 3.6 and a few selected research questions will be presented in section 3.7.

Finally, we invite the interested reader to consult the recent review Emerging multiple issue e-auctions (115) for further details. Some considerations presented here after have been inspired by this paper.

\subsection{A few first observations}

First of all, as stressed by Koppius and van Heck (66), let us point out that ... a multidimensional auction moves beyond the purely distributive aspects of traditional auctions where a bidder's loss is the bid taker's gain, to incorporate integrative aspects that can capture the "money left on the table". The following example, inspired from these authors, illustrates such assertion.

\section{Example: Illustration of the potential integrative nature of multi-attribute auctions.}

Let us consider the procurement of 90 laptops conducted by a middle-sized company. The auctioneer first specifies the requested characteristics of the computer units and then starts a single-dimensional auction. To simplify the example, let us assume that these characteristics can be evaluated according to an ordinal scale noted $\{A, B, C\}$ ( $A$ being better than $B$ and $B$ be- 
ing better than $C$ ). Within this scheme, the minimal requested characteristic is $B$ and the auction ends with a price of $2.000 \mathrm{eu}-$ ros per unit. Let us assume now that the auctioneer was ready to pay an extra 500 euros per unit for a quality $A$ instead of a quality $B$. On the other hand, the winning bidder's cost for producing quality $A$ instead of $B$ is 200 euros per unit. In a multi-attribute context, the bid characterized by 90 laptops at quality $A$ and at a price of 2.350 euros per unit is better for both parties. Let us assume now that, due to production costs, the winning bidder can deliver 100 laptops $(A)$, instead of 90 , at a price of 2.000 euros per unit. The auctioneer acknowledges that 10 additional computers could be needed on the short term but doesn't want to pay more than 5 percent on the total amount he agreed for the 90 computers, i.e., $2.350 \cdot 90 \cdot 1,05 / 100=2.220,075$ euros per unit. With such settings, a bid for 100 computer units $(A)$ at a price of 2.110 euros will be preferred by both parties. These improvements are a consequence of the fact that the parties involved in the auction have different preferences regarding certain dimensions of the product sold.

A second observation, stressed by Kersten et al. (63), is that the presence of two or more issues begins to blur the difference between auctions and negotiations. Indeed, distinctive features of negotiation mechanisms such as for instance logrolling are now supported by multi-attribute auctions. (The first observation about the potential integrative aspect of multi-attribute auctions is another common aspect). Of course, negotiations offer much more possibilities than auctions do. Nevertheless, the two fields can be complementary. Basically, two main approaches can be identified:

\section{- Combinations}

In these settings, auction and negotiation mechanisms are separately conducted. One may first, for instance, imagine a negotiation step, with a selected subset of bidders, in order to establish the first bids, the issues to be considered, ... followed by the auction itself.

On the opposite, an auction could first be played in order to narrow the list of bidders and then be followed by bilateral negotiations with the remaining participants. According to us, this approach is particularly attractive since the negotiation step following the auction permits to choose the final offer in a more flexible way than by using solely the auction. We are convinced of the fact that, even if auctions are supporting 
several attributes, they are not adapted to face the entire complexity of a procurement situation. On the other hand, they offer competitive features that are of the uttermost importance in these situations. It is in this setting that we place our contribution.

These considerations are further completed by Anderson and Frohlich (3) clients do not normally make award decisions on bid days. In the days and weeks that follow the bidding event, buyers examine bid results, review supplier information (such as supplier capability, quality certifications, and manufacturing processes), and sometimes conduct a buyer audit before making final decision. The client does not have to select the lowest bidder. Additionally, Jap. (57) notes that the vast majority of (FreeMarkets procurement) auctions used in the marketplace today do not determine a winner ... and the buyer reserve the right to select a winner on any basis.

\section{- Hybrid forms}

Hybrid forms integrate simultaneously auctions and negotiations. An example of such approach is NegotiAuction (116). The underlying motivation of this system is to be able to take into account, at the same time, the flexibility of negotiations and the competitiveness of auctions.

Finally, the simultaneous management of several attributes increases the cognitive complexity of the bidding process. Improvements of offers can be conducted on several attributes and compensations between these attributes have to be considered. Additionally, the bidders have to know, at least partially, when and how to improve their offers. These questions, related to information revelation (about the buyer preferences, about the status of submitted bids, ...) are, more than ever, topical in this context.

\subsection{Playing with several attributes: different approaches}

Playing with several attributes, instead of the price only, inevitably leads to model the buyer's preferences ${ }^{2}$. In this section we will illustrate a few approaches that have been proposed to deal with these questions.

\footnotetext{
${ }^{2}$ let us stress that, in an auction context, considering the buyer's preferences is not new. Multi-unit (respectively multi-object) auction already integrates buyer's preferences between price quantity pairs (respectively bundle of items).
} 


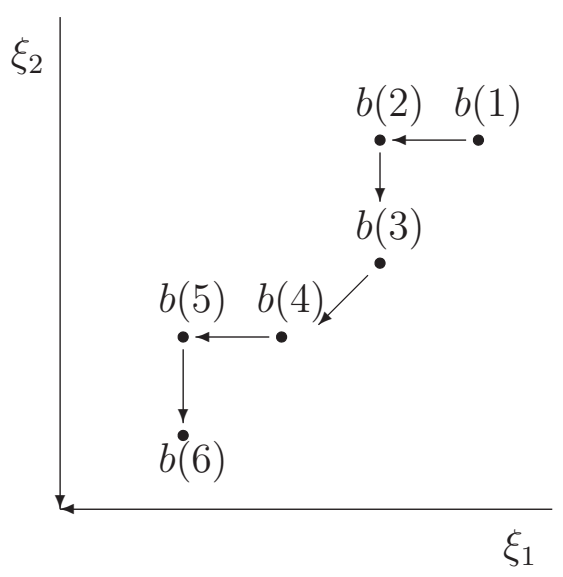

Figure 3.1: The Leap Frog method: example of a potential outcome

Before considering elaborated models that take into account the preferences of the buyer, let us consider two basic extensions of classic auction mechanisms that, precisely, do not rely on these preferences: the leap frog method and what we have called dominance based auctions. Such approaches are, of course, unrealistic since the exploitation of the auctioneer's preferences is at the core of the problem.

The leap frog method (114) is based on the principle that any new bid must be better than the previous one on at least one attribute (each new bid must strictly dominate the previous one). Figure 3.1 illustrates a two dimensional example of such an approach. As already mentioned, the auctioneer's preferences do not intervene in the leap frog method and the winning bid is entirely determined by the bidders. If the simplicity of such mechanism is seducing, it could, however lead to inefficient outcomes.

What we call dominance based multicriteria auctions rely on the principle that any new bid must not be dominated by the previous ones. Conceptually, even if this kind of mechanism seems to be close to the last approach, it relies on a rule that is at the exact opposite. A direct consequence of this framework is that, most of the time, the auction does not end with a single bid, but with a whole set of Pareto Optimal bids. This phenomenon is illustrated in figure 3.2 .

As stressed by Teich et al. (114), a first attempt to incorporate the buyer's preferences is to ask him to provide an auction owner specified bid path that the bidders will follow. Of course, the side effect of such an approach is that it is rather constraining for the bidders. To our knowledge, this method has 


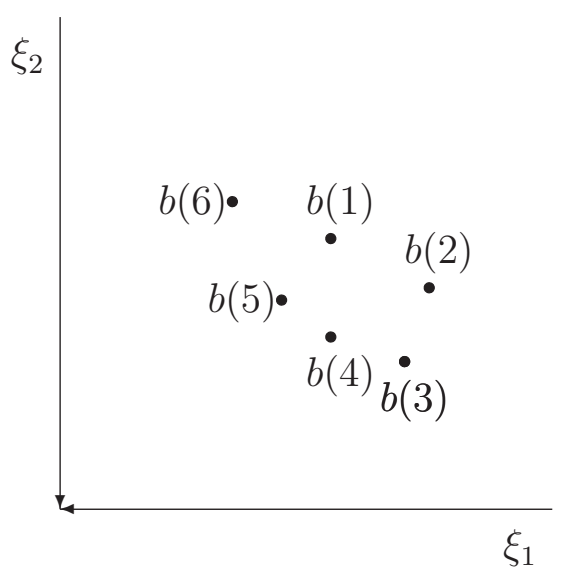

Figure 3.2: Dominance based auction: example of a potential outcome

never been implemented.

In most papers, authors consider multi-attribute utility function to represent the buyer preferences. Usually quasi-linear utility functions are used. In this case, the utility of the buyer is derived as the difference between the price the buyer values the technical specifications of the bid, i.e. all the nonprice attributes $\left(b^{-1}\right)$, referred to as $v\left(b^{-1}\right)$ and the price $p=b^{1}$ that he has to pay to acquire the bid. More formally:

$$
U(b)=b^{1}-v\left(b^{-1}\right)
$$

where $v$ is assumed to be monotonically decreasing in all $b^{j}$. Similarly, when modeling the utility of supplier $s_{i}$, one will assume the following expression:

$$
U_{i}(b)=b^{1}-c\left(b^{-1}, \theta_{i}\right)
$$

i.e. the difference between price of the bid and the cost to produce its related technical specifications $b^{-1}, c\left(b^{-1}, \theta_{i}\right)\left(\theta_{i}\right.$ being a parameter characterizing the cost function assigned to supplier $s_{i}$ ).

Of course, a subcase of the previous expressions is the linear additive utility function; $U(b)=\sum_{j=1}^{q} U^{j}\left(b^{j}\right)$ where $U^{j}\left(b^{j}\right)$ represents the marginal utility associated the $j^{\text {th }}$ attribute and $\omega_{j}$ its related weight.

Approaches based on multi-attribute utility functions have been recently 
criticized by Bellosta et al. (9). In reaction, the authors rather plead for an approach based on reference points. At the beginning of the auction, the buyer is assumed to communicate to the suppliers two particular bids: an aspiration point and a reservation point. The aspiration point reflects the desired values for the different attributes, while, on the contrary, the reservation point expresses the reserve values for all the attributes. By doing so, the preference information and relative importance of criteria are directly expressed in terms of required values on the criteria.

At the end of each bidding round, only bids that dominate the current reservation point are considered as acceptable bids. Then the best bid (of the round) is determined as the one minimizing the maximum (normalized) deviation ${ }^{3}$ from the aspiration point. Then, the reservation point is updated according to the submitted bids. We refer the interested reader to (9) for the details regarding the dynamic property of their mechanisms.

Finally, Teich et al. (116) have adopted the pricing out approach to model the preferences of the buyer in the NegotiAuction system. As stressed by the authors, this method is particularly well-adapted in the context of multiattribute auctions since there is a natural monetary attribute. Furthermore, its relative simplicity makes it easy to understand by the suppliers.

\subsection{A common framework}

The previous section has illustrated a number of distinct approaches to model the buyer's preferences. Of course, the various forms of multi-attribute auctions are distinguishing themselves through a number of points of views. This diversity could make the field of multi-attribute auctions hard to grasp. In a recent review, Teich et al. (115) propose a generic description of multiattribute auctions. In order to clarify the field, we summarize, here below, the six important points they consider:

\section{- Auction Rules}

Before the auction's beginning, the auctioneer has to reveal the auction rules. Is it a one round, multi-rounds or progressive auction? Is the auction opened to all potential suppliers or do they have, first, to satisfy

\footnotetext{
${ }^{3}$ To our knowledge, this min max rule constitutes the first non compensatory approach to multi-attribute auctions.
} 
a qualification criteria? Are the price discriminatory or not? Is there any withdrawal policy ? ...

\section{- Product and Issue/attribute Description, Specification of Quan-} tity Demanded, Reservation Price(s) and Bid Decrement

\section{- Representing Buyer's Preferences over Multiple Attributes}

How to represent the buyer's preferences is a fundamental question in multi-attribute auctions. The various approaches enumerated in section 3.3 have illustrated the diversity of the answers that have already been proposed.

\section{- Winner Determination}

In many auctions, the winner determination can be reduced to detect the seller who offered the bid with the highest utility. Nevertheless, the winner determination problem can become more complex, especially in the case of multiple sourcing (see section 3.7). On the other hand, the winning bid determination constitute another problem; the winning seller may deliver the bid he proposed, a bid characterized by the same utility, ...

\section{- Information architecture}

Information architecture is referred to as what kind of information is available to whom, or when and how it becomes available to whom during the market process (65). Information regarding the current winning bid(s), the buyer's preferences, the number of suppliers taking part in the auction, ...can have impacts on the auction's performances (see section 3.6) and therefore, have to be carefully fixed. For instance, regarding the feedback information the suppliers receive, we can distinguish the following options (ordered according to the underlying information's richness):

- the suppliers receive binary responses saying if their bids belong to the set of winning bids or not.

- the current winning bid is communicated to the sellers

- the submitted bids are ranked according to the buyer's preferences and this ranking is communicated to the suppliers.

- partial information regarding the scoring function (its gradient for instance) used by the buyer is communicated to the sellers. 
- the scoring function used by the buyer is public to the sellers (it could nevertheless be different that the buyer's utility function since misrepresentation may be profitable (65))

\section{- Closing Rules}

Traditionally, two closing rules are considered. The first one states that the auction ends as soon as no new bid is received during a specific waiting period (for instance, 120" in (112)). The second rule imposes a predefined time limit. However, as already stressed in chapter 1, this rule suffers from the snipping effect and therefore, the time limit is often extended if an abnormal high bidding activity is detected during the last few minutes (or the last hour) before the auction's end.

\subsection{How to characterize the mechanisms ?}

Facing the variety of multi-attribute auctions, a fundamental concern is to be able to compare and characterize them. Here below, we present a non-exhaustive list of evaluation criteria ${ }^{4}$.

\section{- Total cost to the buyer / buyer's surplus}

As stressed by Teich et al. (115), total cost to the buyer is not a measure that is well-adapted to the context of multi-attribute auctions; since the non-price attributes may vary considerably from one bid to the other, comparing different values of the total cost is not really meaningful. When using a pricing out approach this argument is somewhat softened since the cost is corrected by the value of the non-price attribute. Finally, another option is to measure the buyer surplus, i.e. $v\left(b^{-1}\right)-b^{1}$ (111).

- Revenue / profit to the winning seller

Similarly to the arguments presented in the previous point, the revenue of the winning seller is not an adapted measure to evaluate multiattribute auctions. In such contexts, we will rather use the seller's surplus $b^{1}-c\left(b^{-1}, \theta_{i}\right)$. However, from a practical point of view, these costs, being a private information of the supplier, are often unknown to the person in charge of evaluating the auction.

\footnotetext{
${ }^{4}$ To illustrate them, we will consider that both the utility of the buyer and the winning seller $s_{i}$ are quasi-linear multi-attribute utility function (see section 3.3)
} 


\section{- Pareto Optimality}

An outcome is Pareto Optimal if and only if no other deal exists, which is preferred by at least one of the two agents, either the buyer or the selected winning supplier, and for which the other agent is at least indifferent (121).

\section{- Social welfare and Allocative efficiency}

Social welfare is measured as the total of the buyer and winning supplier's surplus:

$$
\left(v\left(b^{-1}\right)-b^{1}\right)+\left(b^{1}-c\left(b^{-1}, \theta_{i}\right)\right)=v\left(b^{-1}\right)-c\left(b^{-1}, \theta_{i}\right)
$$

Let us note that this measure is independent of the price of the winning bid $b^{1}$. Allocative efficiency evaluates if the considered mechanism leads to an outcome that maximizes the social welfare.

\section{- Incentive compatibility}

In the context of multi-attribute auctions, one may wonder if the bidders or the buyer could derive benefits from misrepresenting their preferences. If it can be theoretically proven that it is not the case, the mechanism proposed will be judged as incentive compatible.

\section{- Speed of convergence}

Parameters such as the decrement step(s) strongly influence the duration (and the outcomes) of the auction. Therefore, their tuning is a critical step.

\section{- Computational efficiency}

Contrary to traditional auctions, multi-attribute auctions may involve complex tools that are likely to influence the auction. For instance, due to the cognitive complexity of bidding in a multi-attribute auctions, bidding support systems have been proposed. Another problem that is commonly cited is related to the winning bid selection. In an auction context, where the dynamical aspect is crucial, the computational efficiency of these approaches is of the uttermost importance.

- ...

Of course, other aspects, such as for instance fairness or simplicity have to be considered. 


\subsection{A few experimental results}

As already stressed, multi-attribute auctions have been theoretically studied by a few authors. These contributions will not be detailed in this work and we refer the interested reader to $(112 ; 12 ; 65 ; 26)$ for a detailed analysis. Parallel to these findings, some researchers have chosen to experimentally study the subject. In this section, we outline some of their works (based on the chronological order of their publication). To our point of view, the question they address illustrate the variety of research directions attached to this field. Besides their empirical results, we will also summarize the experiments' settings; this will illustrate a number of points presented in the previous sections.

\section{Experiments conducted by Bichler}

Martin Bichler (12) seems to be the first one to have studied multiattribute auctions experimentally; he considered the use of three different multi-attribute auction formats (first score sealed bid, second score sealed bid ${ }^{5}$ and multi-attribute English auctions) in the context of OTC financial derivatives trading.

The experiments were conducted in May and October 1999 at the Vienna University of Economics and Business Administration (participant were MBA students). Financial derivatives were evaluated according to their strike price and implied volatility. Both single and multi-attribute auctions were conducted. In the multi-attribute context, the bidders could simultaneously play on both attributes. The preferences of the auctioneer were represented by a linear two-attribute function (this function was public to the participants). In single attribute auctions, the strike price was held constant and the bidders could only play on the implied volatility. We refer the interested reader to (12) for a detailed description of the experimental settings.

The aim of these tests was to answer the following questions:

1. Are the equilibrium values achieved in a multi-attribute auction higher compared to single-attribute auctions with respect to the underlying

\footnotetext{
${ }^{5}$ In the case of second score sealed bid auctions, the winning bidder is the one who proposed the bid with the highest score. However, he only has to supply the buyer with a bid the score of which is equal to the second highest score. Practically, Bichler proposes to transform the gap $S\left(b_{(1)}\right)-S\left(b_{(2)}\right)$ into monetary units
} 
scoring function of the bid taker?

2. Are all multi-attribute auction formats payoff equivalent?

3. Are multi-attribute and single-attribute auctions efficient?

As expected the answer to the first question was positive; multi-attribute auctions achieved higher scores than single-attribute auctions. As stressed by Bichler, one explanation for this result is that in a multi-attribute auction, a bidder has more possibilities for improving the value of a bid for the bid taker, sometimes even without increasing her own costs. Furthermore, the obtained results have led to reject the revenue equivalence assumption; first score sealed bid auctions achieved higher revenue than multi-attribute English auctions and multi-attribute English auctions achieved higher revenue than second score sealed bid auctions (let us note that the same order appeared in single-attribute auctions). Additionally, the strategic equivalence between second score sealed bid auctions and multi-attribute auctions had to be rejected (and was also rejected in the single-attribute context). The distinctive features of the experiment can partially explain these outcomes (see (12) for a detailed discussion). Finally, no significant difference between single-attribute and multi-attribute efficiency could be found (over the three auction formats).

\section{Experiments conducted by Koppius and van Heck}

The works of Koppius and van Heck (66) address another fundamental question: how parameters like the length of the auction and the information architecture $^{6}$ can affect the performance (in terms of pareto optimality and winner efficiency) of multi-attribute auctions?

To perform the tests, the authors consider the chemical industry context; 4 suppliers of hydrochloric acid are competing by means of multi-attribute bids in a multi-round English multi-attribute auction. Three attributes are considered; the price, the delivery time $t$ and the contamination percentage $c$. The preference of the auctioneer are represented by the following multiattribute function: $U(b)=v_{1} e^{-c}+v_{2} e^{-t}-p^{2}$.

In order to model the two aforementioned effects, they consider:

\footnotetext{
${ }^{6}$ Koppius (65) defines information architecture as what kind of information is available to whom, or when and how it becomes available to whom during the market process
} 
1. auctions of 2 rounds versus auctions of 4 rounds

2. restricted information architecture versus unrestricted information architecture.

In the restricted information architecture, the bidders only know the overall highest bid and bidder at the end of each round. On the contrary, in the unrestricted information architecture setting, bidders additionally know all the bids submitted during the last round as well as their relative utility compared to the highest one.

Efficiency is measured by two indices: the number of efficient bid and the distance between the winning bid and the efficient bid. Similarly, Pareto optimality is measured both by the number of pareto optimal bids and by the number of pareto-improving bids that existed at the end of the auction. The analysis of these measures leads to the following conclusions:

- unrestricted information architecture increases the pareto optimality and winner efficiency

- playing the auction in 4 round instead of 2 improves the pareto optimality but has no significant effect on efficiency

- the combination of the two effects does not lead to significant market improvements.

The two first results presented here after are intuitive. However the fact that the combination of the factor does not lead to significant market improvements is surprising. The authors claim that this suggests that there may be a phenomenon of information saturation at work: beyond a certain point, more information does not improve market performance further.

\section{Experiments conducted by Chen-Ritzo et al.}

The experiments conducted by Chen-Ritzo et al. (26) further complete the previous analysis. The authors compare the results of English multiattribute auctions with those of traditional English auctions and, additionally, measure the effect of the bidder's experience on the outcomes.

Therefore they consider the procurement of an abstract object characterized by three attributes: price, quality and lead time. Both the buyer's 
utility (which is represented by a computer) and the (human) bidder's profit are assumed to be quasi-linear in price. The bidders who participated to the experiment where undergraduate and graduate students from the Pennsylvania State University. In order to measure the effect of the bidder's experience on the auction's outcome, a distinction has been made between inexperienced and experienced bidders. Inexperienced bidders were students who had not previously participated to the experience. On the contrary, experienced bidders were students who had already previously participated to the experience. For each setting, nine auctions with 3 bidders were organized.

In the traditional English auction setting, reservation levels for the different attributes are first communicated to the bidders. Then the competition is restricted to the price. At each time, the bidders are informed of the time remaining in the auction, the current best bid and their bid status.

In the English multi-attribute auction setting, the buyer's utility function is not directly communicated to the bidders (the authors, indeed, criticizes this way of doing since this information can be private or can evolve during the auction). On the contrary, the following information is provided to the bidder:

- the last price difference, i.e. the amount by which the bidder needs to reduce the price of his last bid in order to beat the current best bid

- the marginal quality, i.e. the buyer's marginal utility for improvement in quality at the bidder's most recently placed bid

- the marginal lead time, i.e. the buyer's marginal utility for improvement in lead time at the bidder's most recently placed bid

Additionally, due to the complexity of the bidding process, a bidding support tool was at the bidder disposal. Finally, the auction outcomes were quantified in terms of buyer utility and seller profit.

These experiences have led to the following main conclusions: the use of multi-attribute auctions increases buyer utility and does not degrade (and occasionally increases) seller profits. Furthermore, performance in the multiattribute auction improves with experience. 


\section{Experiments conducted by Strecker}

Recently, Strecker (111) has studied the impact of preference revelation in multi-attribute auctions. Therefore, he considered the procurement of an abstract object characterized by three negotiable attributes: the price and 2 abstract qualitative, non price attributes (with 6 different levels). Both buyer and supplier utilities are quasi-linear in price. Contrary to other approaches, Strecker consider real-time bid submission (versus multi-round bid submission). The auction ends after 120" of inactivity. Participants are undergraduate and graduate students (mainly from the Department of Economics and Business Engineering) from the Universitaet Karlsruhe (Germany). Every participants will play both role: supplier and buyer (in each auction, there are exactly 4 suppliers and 1 buyer). At each time, buyers know the current best bid and the time remaining before the end of the auction.

Strecker study one main effect: the impact of preference revelation. Therefore, he consider two distinct scenario:

- Full disclosure: the buyer reveals his scoring rule

- Non disclosure: the buyer does not reveal his scoring rule. Instead very general information is communicated to the bidder (111):

- given identical qualitative attributes, the buyer prefers the bid with the lower price

- the buyer prefers a higher quality level to a lower quality level within a a qualitative attribute all over attributes being equal

- a lower quality level in one qualitative attribute can be compensated by a higher quality level in the other qualitative attribute

- likewise it is possible to compensate a lower quality level by a a lower price and vice versa

The performance of the auction outcome is measured in terms of allocational and pareto efficiency (see (111) for details). Tests have led the author to the following conclusions:

- In a multi-attribute English auction, efficiency is higher if the buyer's preferences are known by the suppliers prior to the auction. If preferences are revealed, the average efficiency is close to the theoretical maximum. Independent of the preference revelation, efficiency is quite high in both treatments. 
- Suppliers successfully use the additional information about buyer preferences and make (partly significantly) more profits, but not at the expense of the buyer, whose utility does increase slightly yet nonsignificantly with preference revelation.

- In a multi-attribute English auction, Pareto efficiency is higher if the buyer's preferences are known by the supplier prior to the auction (Pareto efficiency depends on the performance of individual bidders).

\subsection{A few selected additional research questions}

As shown in the previous sections, multi-attribute auctions constitute an active research field. In order to complete our presentation, here are a selection of research directions:

\section{- Bidding support systems}

In multi-attribute auctions, bidding is much more complex than the simple beat the quote rule used in traditional auctions. Therefore, several authors $(26 ; 111)$ have already stressed the need of adequate bidding support systems to insure a proper development of multi-attribute auctions. For instance, in NegotiAuction, Teich et al. (116) have integrated a module referred to as 'Suggested price' to support the bidders.

\section{- Generalization of auction mechanisms}

As pointed out in chapter 1, auction mechanisms have deeply evolved. Nowadays, we speak about multi-unit, multi-item (i.e. multi-object or combinatorial) or multi-attribute auctions. Nevertheless, the potential combinations of these recent extensions open even more new research directions.

In this context, Bichler et al. (14) have recently proposed a global overview of the field. The authors proposed to classify auction mechanisms according to three criteria:

- number of different items (single or multiple heterogenenous items)

- number of negotiable attributes (single or multiple attributes)

- quantity for each item (single or multiple units) 
Table 3.1: Multidimensional auctions

\begin{tabular}{|ccc|c|}
\hline nb diff. items & nb. attr. & quantity & Description \\
\hline single & single & single & Traditional auctions. \\
single & single & multiple & Multi-unit auctions. \\
multiple & single & single & Combinatorial auctions. \\
single & multiple & single & Multi-attribute auctions. \\
multiple & multiple & single & Multi-attribute comb. auctions. \\
single & multiple & multiple & Multi-item multi-attribute auctions. \\
multiple & single & multiple & Multi-item comb. auctions. \\
multiple & multiple & multiple & Multidimensional auctions. \\
\hline
\end{tabular}

Table 3.1 summarizes these $\left(2^{3}\right)$ combinations. The four first instances are well-known auction formats.

Multi-attribute combinatorial auctions constitute a first extension to multi-attribute auctions. In this context, several heterogeneous items, characterized by several attributes, are simultaneously purchased.

Multi-item multi-attribute auctions address the problem of multiple sourcing where (indivisible) bids are characterized by multiple attributes ${ }^{7}$. In this context, a fundamental problem is the winners determination. One solution has been recently proposed by Bichler et al. (14):

$$
\begin{gathered}
\max \sum_{s_{i} \in S} \sum_{b_{i_{j}}}\left(q \left(b_{i_{j}} \cdot S\left(b_{i_{j}}\right) \cdot x_{i_{j}}\right.\right. \\
d_{\text {min }} \leq \sum_{s_{i} \in S} \sum_{b_{i_{j}}} q\left(b_{i_{j}}\right) \cdot x_{i_{j}} \leq d_{\max } \\
\sum_{s_{i} \in S} \sum_{b_{i_{j}}} q\left(b_{i_{j}}\right) p\left(b_{i_{j}}\right) x_{i_{j}} \leq R \\
\sum_{j} x_{i_{j}} \leq 1, \forall s_{i}
\end{gathered}
$$

\footnotetext{
${ }^{7}$ Until very recently, research on multi-attribute auctions has been exclusively focused on the sole sourcing problem. This is due to the fact that, in procurement contexts, the contracts negotiated are characterized by a high asset-specificity (DoD contracts for instance). Nowadays, multi-attribute auctions are used for the purchase of less specific products, which are traded for larger quantities.
} 


$$
x_{i_{j}} \in\{0,1\}
$$

where $s_{i}$ denotes the $i^{t h}$ supplier, $b_{i_{j}}$ is the $j^{t} h$ bid proposed by supplier $s_{i}, q\left(b_{i_{j}}\right)$ represents the quantity of bid $b_{i_{j}}$ and $S(b)$ is the score associated to bid $b$. The objective function of this integer program ensures that the overall score is maximized under the following constraints; the demand is satisfied (first constraint), the reserve price $R$ is not exceeded (second constraint) and at most one bid per supplier can be selected (third constraint).

Multi-item combinatorial auctions combines the distinctive features of multi-unit and combinatorial auctions. Finally multidimensional auctions constitute the most general case; all dimensions are negotiable.

\section{- Bidding languages}

The need of adequate bidding languages has been emphasized in the context of combinatorial auctions. Similarly, during multi-attribute auctions, bidders may also be confronted with the problem of the bids expressivity.

To illustrate this purpose let us consider the following example (inspired from (14)): a company is organizing a multi-attribute auction to purchase 50 computers. Four attributes are considered: the price, the size of the hard disk, the processor's speed and the warranty. A famous computer's dealer decides to participate to the auction; he can offer three different sizes for the hard disk (20 GB, 30 GB or $40 \mathrm{~GB}$ ), three different processor's speed $(1 \mathrm{MHz}, 1.5 \mathrm{MHz}$ or $2 \mathrm{MHz})$ and two warranty conditions ( 1 year warranty or 3 years warranty). Consequently, this supplier can propose 18 distinct configurations. In order to facilitate the bidding process, Bichler et al. (14) proposes to model offers using the following formulation:

$$
\tilde{p}\left(b_{i}\right)=q\left(b_{i}\right)\left(p\left(b_{i}, q\left(b_{i}\right)\right)+\sum_{j} f_{i j}\left(v_{i j}\right)\right)
$$

In other words, the price $\tilde{p}\left(b_{i}\right)$ related to a specific configuration $b_{i}$ is equal to the offered quantity $q\left(b_{i}\right)$ multiplied by the base price per unit (as a function of the quantity) $p\left(b_{i}, q\left(b_{i}\right)\right)$ plus a premium related to the values of the different attributes $v_{i j}$. 


\subsection{Conclusion}

In this chapter, we have presented some of the recent developments and research questions attached to the field of multi-attribute auctions. More than ever, topics such as information revelation, preferences elicitation or bidding support systems are of the uttermost importance.

As already stressed, most of the related contributions have less than five years old. Nevertheless, the increasing number of (academic or industrial) papers and talks show that it is likely to remain an active field of research within the next years.

Multi-attribute auctions constitute an interesting tool for the automation of procurement activities. Nevertheless, to our point of view, they cannot replace the whole sourcing process. On the contrary, they must be viewed as a first competitive step followed by a more detailed negotiation. It is in this spirit that we have explored the field of multicriteria auctions. 


\section{Part II}

\section{New developments}




\section{Chapter 4}

\section{Multicriteria auctions: a theoretical framework}

\subsection{Introduction}

As stressed in chapter 3, several authors have, recently, proposed and studied auction mechanisms supporting multidimensional bids. These approaches rely on a variety of distinct models for bids comparison.

In this chapter, our motivation is somewhat different; we want to analyze such mechanisms without making an explicit reference to a specific preference structure. On the contrary, our starting point will be to state that, when comparing two bids, only 3 situations can happen: one of the bids is preferred to the other, the two bids are indifferent or the two bids are incomparable. By working with a general preference structure $(P, I, J)$, our aim is to study the field at a more abstract level. Of course, due to the poverty of our assumptions, we do not expect to obtain refined results, but rather to get a global picture of the problem.

The consideration of a general preference structure $(P, I, J)$ is, of course, motivated by the works done within the so-called French school of multicriteria decision aid. The fact that two bids might be incomparable is, however, not obvious since all existing auction mechanisms are precisely based on the total comparability assumption; any pair of bids is comparable. This distinctive feature is peculiar to our approach and certainly constitutes its main originality. Consequently, this point will be thoroughly discussed in the next section. 
Furthermore, from a multicriteria decision aid point of view, this chapter offers an interesting perspective since it integrates a dynamical component to the classic choice problematic (99). Yet, it is worth noting that there exists a fundamental difference between the bid selection in an auction context and the selection of alternatives in a multicriteria decision aid problem. In an auction context, the competition between the sellers is at the core of the problem, the set of bids received by the auctioneer evolves during the auction and the bids selection is likely to accentuate the competition. On the contrary, in a multicriteria decision aid context, the analysis of the decision is at the core of the problem (preferences learning / tuning, problem structuring, ...), the set of potential alternatives is, in many cases, fixed (or, at least will not change too much) and the selection of alternatives is related to an exploration phase where good compromises alternatives are outlined. These two positions are not always compatible and the auction perspective will be predominant in this work.

Of course, the proposed framework relies on a variety of (explicit or implicit) hypothesis that will be outlined in the next pages. Among them, the following assumption has fundamentally influenced our approach:

Assumption 1 Once a bid has been beaten, it remains beaten until the auction's end.

From an auction perspective this requirement seems to be natural. Nevertheless, from a multicriteria decision aid point of view this constraint is less intuitive since, as already stressed, the emphasis is laid on the problem exploration / understanding. In an auction context, we claim that it is hard to justify to a potential seller that one of his bid - that has been beaten before - is, once again, considered as a potential winning bid. Especially if, meanwhile, he has been pushed to propose other bids ${ }^{1}$.

This chapter is organized as follows: the second section is dedicated to the motivation of the existence of potential incomparability between multidimensional bids. Then, the third section will be devoted to the problem's formalization. The main ideas, on which relies our theoretical framework, will be introduced, then the bids comparison problem will be discussed to, finally, be able to define the notion of multicriteria auction and its related concepts. In the end, the framework will be criticized and its distinctive features will be outlined. The fourth section is devoted to the theoretical properties of

\footnotetext{
${ }^{1}$ here we do not consider bids' withdrawal policy.
} 
the winning bids. Our attention will, here, be focused both on the winning set, on the competition between the sellers and its impact on the winning bids. Section 4.5 will present a pedagogical example of a procurement auction based on the Electre I method. The notions, previously introduced, will, there, be widely illustrated. Extensions of multicriteria auctions to other auction formats, such as Vickrey and Dutch auctions, will then be discussed in section 4.6. These considerations will be followed by particularizations of our framework to existing approaches in section 4.7. In this way, unicriterion, dominance based and multi-attribute auctions will be outlined. In section 4.8 general questions related to the use of multicriteria auctions will be treated . Finally, a conclusion will summarize our results and present directions for future researches.

To conclude, let us stress that this chapter is an extended version of the papers "Multicriteria Auctions without full comparability of bids" (39) and "Multicriteria Auctions: an introduction" (36).

\subsection{The management of incomparability between multidimensional bids}

The management of incomparability between multidimensional bids constitutes the main originality of our contribution. However, this distinctive feature is rather surprising for most people involved in auction theory. Indeed, in such a context, all pairs of bids are assumed to be comparable. As a consequence, every new offer can be compared to previous ones and, consequently, winning bids can be univocally determined.

Influenced by the research done within the so-called French school of the multicriteria decision aid community, we have been pushed to consider such an idea. Of course, we will not re-explain, here, all the motivations that have convinced us not to limit ourselves to the preference and indifference relations. Nevertheless, a number of arguments seem to be particularly meaningful within this auction context.

First of all, the compensatory nature of aggregation methods such as those based on a multi-attribute utility function is a critical point. In this context, the details of the bids are 'hidden' by an aggregated value. Therefore, a bid $b$ can be indifferent or, worst, be preferred by $\tilde{b}$ even if these two bids have totally different natures. Within a competitive context, such situations can be hard to justify to potential sellers. 
In the same spirit, one may also stress that slightly changes in the preferences of the auctioneer can lead to finally select winning bids that are diametrically opposed. This effect is illustrated on figure 4.1. Using a multiattribute linear utility function leads to choose $b_{1}$ as the winning bid (the continuous line representing the set of iso-utility points). However, a slightly change in the weights of the utility function leads to choose $b_{2}$ instead of $b_{1}$ (these two bids are, nevertheless, structurally opposed). Finally, no weight instance can lead to consider $b_{3}$ as a winning bid - even if this bid is not dominated.

Moreover, due to the complexity of the items traded, one can imagine that the auctioneer does not want to restrict himself to a unique 'best' winning bid, but, on the contrary, wants to get several good offers. In this way, the final winning bid selection is not confided to an idealized theoretical model and the auctioneer may negotiate with a subset of selected sellers in order to point out the winner and to fix the terms of the contract. By doing so, the multicriteria auction is viewed as a first competitive step which is followed by one-to-one negotiations. This point will be further studied in section 4.3.4.

Finally, in some problems (see examples in chapter 6), the bids are not homogeneously distributed in the bidding space. On the contrary, several "zones" characterized by a high bidding activity can be identified. In what follows, these "zones" will be referred to as bidding niches (this notion will be formalized in chapter 6 . The use of partial pre-orders for the pair-wise comparison of offers will permit us to manage different bidding niches during the same auction. As a consequence, at the end, several winning bids may be proposed to the buyer; one for each niche. Figure 4.1 illustrates a situation were two bids $b_{1}$ and $b_{2}$ characterize two bidding niches.

To conclude, let us underline that we do not claim that incomparability must be taken into account in any auction; this is above all depending on the auctioneer preferences. Anyway, in our framework, we let this door open.

\subsection{Problem's formalization}

In this section, we will introduce the main concepts and definitions that will be used thorough the present chapter. In fine, this will lead us to formalize the notion of multicriteria auction. 


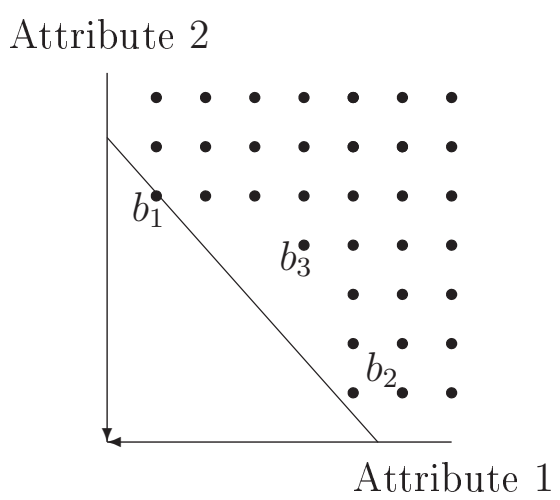

Figure 4.1: Example of a 2-attributes context with two bidding niches.

\subsubsection{Main concepts and definitions}

As already stressed in the previous chapter, we will consider here the context of a reverse auction: p sellers are competing together to sell an object, service, contract, ... to a unique buyer. This situation is not restrictive and the following formalism can easily be adapted to cover other situations. Furthermore, to avoid any ambiguity we will use, from now on, the term item to refer to what is being sold.

Let $B$ denote the buyer, let $S=\left\{s_{1}, s_{2}, \ldots, s_{p}\right\}$ be the set of the $p$ sellers participating to the auction and let $I$ be the set of items corresponding to the buyer's specifications.

$B$ decides to evaluate the bids on $q$ criteria noted $\left\{\xi^{1}, \xi^{2}, \ldots, \xi^{q}\right\}, \xi^{j}$ : $I \rightarrow E^{j}$ (These criteria are, of course, public to the sellers). Without loss of generality, we may assume that all these criteria have to be minimized. Right now, no further assumptions are made about the nature of the sets $E_{j}$.

For simplicity reasons, each seller $s_{i}$ is assumed to have a single item $v_{i}(\in I)$ to propose to $B$ (this assumption will be later relaxed). Let us note $\xi\left(v_{i}\right)=\left\{\xi^{1}\left(v_{i}\right), \xi^{2},\left(v_{i}\right), \ldots, \xi^{q}\left(v_{i}\right)\right\}$ its evaluation according to the different criteria. By extension, $\xi\left(v_{i}\right)$ will be referred as the valuation of seller $s_{i}$. Intuitively, $\xi^{j}\left(v_{i}\right)$ represents the lowest value that seller $s_{i}$ could propose for the attribute $j$. Of course, $s_{i}$ will offer bids that are dominated by $\xi\left(v_{i}\right)$ or eventually equal to it $\left(b_{i} \preceq \xi\left(v_{i}\right)\right)$ (In this way, he could realize a positive margin). Otherwise, if $s_{i}$ wins the auction, he will not be able to provide the item corresponding to the winning bid. 
Definition 12 Let $\delta_{i}=\delta\left(\xi\left(v_{i}\right)\right)=\left\{b_{i} \in \prod_{j=1}^{q} E^{j} \mid b_{i} \succeq \xi\left(v_{i}\right)\right\}$ be the bidding set of $s_{i}$.

Intuitively, $\delta_{i}$ represents all the bids that seller $s_{i}$ could offer. Of course, we assume here that any bid $b_{i} \in \delta_{i}$ has an economic meaning. Let us note $\delta=\bigcup_{i=1}^{p} \delta_{i}$ the set of all bids that could be offered by any seller participating to the auction. By abuse of language, $\delta$ will be referred as the bidding space. Let us remark that, since each bidder $s_{i}$ only knows his own evaluation $\xi\left(v_{i}\right)$ and the buyer does not know any evaluation, the bidding space remains a theoretical notion that will practically never be observed by any auction participant.

\subsubsection{Bids comparison}

As motivated in chapter 2, comparing alternatives that have a multicriteria nature leads to consider, at least, three situations: preference $(P)$, indifference $(I)$ or incomparability $(J)$. Despite the fact that many models do not take into account potential incomparabilities between alternatives, we already have argued in favor of outranking approaches that, on the contrary, support this distinctive feature.

Even if we try to keep the preference structure $(P, I, J)$ as general as possible, some assumptions have to be imposed in order to remain consistent with the auction context.

Assumption 2 Mixed transitivity assumption: $a(P \cup I) b, b \preceq c \Rightarrow$ $a(P \cup I) c$

First of all, let us note that a direct consequence of this assumption is that $a \preceq b \Rightarrow a(P \cup I) b$, i.e. any improvement on at least one criterion leads to an offer $a$ that is at least as good as $b$. Furthermore, as it is frequently done in the multicriteria decision aid community, we will note $\mathcal{S}=(P \cup I)$.

The mixed transitivity assumption insures some consistence between the preference and indifference relations and the natural dominance relation. A direct corollary of this constraint is that if an offer $b_{i}$ is such that $b_{i}(P \cup$ $I) \xi\left(v_{j}\right), b_{i}$ will be preferred or indifferent to any offer that seller $s_{j}$ could propose. As illustrated in the next example, this assumption is not always satisfied. 
Example 11 A preference structure combining the dominance relation with aspects of multi-attribute linear utility approaches.

Let us consider the following preference structure:

- $a P b \Leftrightarrow a \prec b$ or $L_{1}(a, b) \leq k, U(a)=\sum_{k=1}^{q} w_{k} \cdot a^{k}<\sum_{k=1}^{q} w_{k} \cdot b^{k}=$ $U(b)$

- $a I b \Leftrightarrow L_{1}(a, b) \leq k, U(a)=\sum_{k=1}^{q} w_{k} \cdot a^{k}=\sum_{k=1}^{q} w_{k} \cdot b^{k}=U(b)$

- $a J b$ otherwise

Intuitively, this is nothing else than the "classic" dominance relation enriched by a multi-attribute linear utility function defined on the neighborhood of the considered point. (See figure 4.2)

Even if such an approach could be motivated within an auction context, it violates the aforementioned property. To illustrate this statement let us consider the following example: $k=3, w_{1}=w_{2}=0.5, \xi\left(v_{1}\right)=(10,10), \xi\left(v_{2}\right)=$ $(11,9.5)$. It is easy to verify that $\xi\left(v_{1}\right) P \xi\left(v_{2}\right)$ since $L_{1}\left(\xi\left(v_{1}\right), \xi\left(v_{2}\right)\right)=2.5 \leq 3$ and $U\left(\xi\left(v_{1}\right)\right)=10<10.25=U\left(\xi\left(v_{2}\right)\right.$. On the other hand, $s_{2}$ could propose $b_{2}=(13,9.5) \in \delta_{2}$ such that $\xi\left(v_{1}\right) J b_{2}$ and $b_{2}$ could not be beaten even if it is the case for $\xi\left(v_{2}\right)$. Consequently, $\xi\left(v_{1}\right)(P \cup I) \xi\left(v_{2}\right), \xi\left(v_{2}\right) \preceq b_{2}$ but $\xi\left(v_{2}\right) \neg(P \cup I) b_{2}$.

In what follows, we will assume that the preference structure $(P, I, J)$ is public to the sellers. This assumption relies on the fact that any seller involved in the auction must know how to improve his bids. Weaker assumptions may be considered, but this goes behind this theoretical framework.

\subsubsection{Multicriteria auction}

With reference to classic English auctions, we will define a multicriteria auction as a succession of offers such that each new bid may not be preferred or indifferent to a bid that has already been proposed (for simplicity, we will assume that bids are received one by one). More rigorously: 


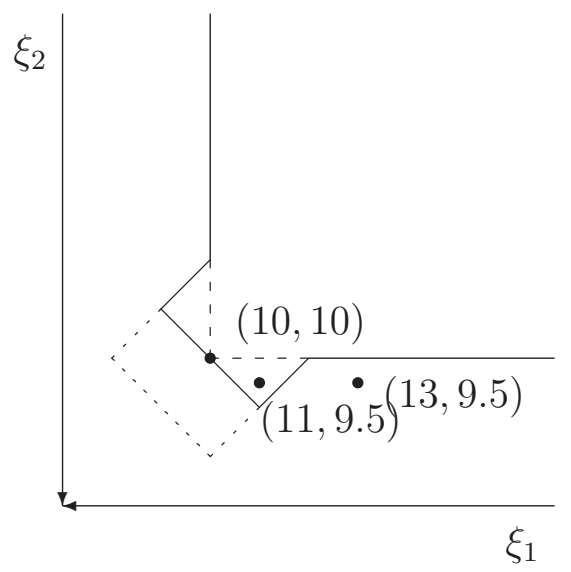

Figure 4.2: Example of an auction context where the mixed transitivity assumption is not satisfied

Definition 13 Let $O$ be a succession of offers: $O: \mathbb{N} \rightarrow \delta: k \rightarrow b(k)$ and $H_{O}(k)$ its history at time $k: H_{O}(k)=\cup_{l=0}^{k} b(l)$. A multicriteria Auction (MA) is a succession of offers satisfying the following bidding rule: at each time $k$, bids b such that $\exists \tilde{b} \in H_{M A}(k-1): \tilde{b}(P \cup I) b$ are not accepted.

This rule seems to be the weakest requirement we can impose on the succession of offers to represent a multicriteria auction (MA) (since in a classic unicriterion auction the price of any new bid must be, at least, lower or equal than the current price minus a decrement step. The parallel between indifference situation and decrement step will be further analyzed in the next chapter).

Let us note that, by definition, no pair of indifferent bids will appear during the auction process. Another direct corollary of the auction rule is that no bid cycles $(b(1) \mathcal{S} b(2) \mathcal{S} \ldots \mathcal{S} b(n) \mathcal{S} b(1))$ can appear in the auction history (even if the underlying $\mathcal{S}$ relation is not acyclic). Finally, let us note that we have defined a multicriteria auction as being an extension of a classic English auction. Using the term English multicriteria auction is more rigorous but, as it is often the case in classic auction theory, we will voluntarily maintain the analogy between the terms Auction and English Auction.

Finally, we will state that a multicriteria auction will end when no more seller proposes new bids. Let $\bar{H}_{M A}(k)$ be defined as follows: 


$$
\bar{H}_{M A}(k)= \begin{cases}H_{M A}(k) & \text { if } b(k) \text { exists } \\ \bar{H}_{M A}(k-1) & \text { otherwise }\end{cases}
$$

A multicriteria auction ends when no seller proposes any new bids:

Definition 14 A multicriteria auction $M A$ ends at time $K$ if $\forall k \geq K, \bar{H}_{M A}(k)=$ $\bar{H}_{M A}(K)$

Intuitively no time limit is imposed here: neither on the length of the auction, nor on the time separating two bids. On the contrary, we implicitly assume that every bidder $s_{i}$ continue to offer new bids $b_{i}$ until he is among the winning bidders or he has reached $\xi\left(v_{i}\right)$. (This assumption will be explicitly studied in section 4.4.2). Therefore, the auction will end whenever all the bidders have stopped offering new bids. This idealized statement is of course related to the theoretical nature of this framework. In practice, time limits have to be considered.

Due to the fact that the $\mathcal{S}$ relation might be a partial pre-order, the selection of the winning bids is not obvious. In an auction context, we argue the whole set of non-preferred bids from the auction history has to be taken into account:

Definition 15 At each time $k$, the winning set of bids associated to the multicriteria auction $M A$, noted $W S_{M A}(k)$ is defined by:

$$
W S_{M A}(k)=\left\{b \in H_{M A}(k) \mid \nexists \tilde{b} \in H_{M A}(k): \tilde{b} P b\right\}=N P S\left(H_{M A}(k)\right)
$$

The winning set $W S_{M A}(k)$ of a multicriteria auction MA at time $\mathrm{k}$, is the set of non preferred bids from its history, also noted $N P S\left(H_{M A}(k)\right)$. A direct corollary of both this definition and the one of a Multicriteria auction is that all bids belonging to $W S_{M A}(k)$ are mutually incomparable. By convention, we will say that a bidder is in the winning set if, at least, one of its offer is in it. Moreover, if MA is a multicriteria auction and $\mathrm{K}$ its time of end, we note $W S_{M A}=W S_{M A}(K)$

\subsubsection{Discussion}

Having presented the main concepts and definitions on which relies our theoretical framework, it is now appropriate to comment some of its distinctive features. 
- Item's valuation: private, interdependent or common values. A central distinction that is made in "classic" auction theory refers to the item's valuation of the bidders. This point is, first of all, strongly linked to the probabilistic and game theoretical aspects of the studied mechanisms and can have important effects on the related theoretical results.

In our framework, since we have assumed that every bidder $s_{i}$ knows $\xi\left(v_{i}\right)$ before the auction's beginning and that this value does not depend on the other bidders' valuations, we refer ourselves to the private values model. Of course, this assumption has been motivated for simplicity. However, from a practical point of view, the particular nature of the items sold and the procurement situation rather plead for correlated or common values assumptions. Extensions of the present framework to these situations go nevertheless beyond the goals of this chapter.

\section{- Information revelation.}

As stressed in the previous chapter, information revelation is a key question related to multi-attribute auctions. In our context, we have assumed that the preference structure of the auctioneer is public to the sellers. On the one hand, bidders need to know how to improve their bids. On the other hand, most of the time, the auctioneer doesn't want to reveal his preferences. In this context, the strongest assumption has been chosen for simplicity reasons. Similarly, we assume perfect feedback information; every bidder knows the attribute values of all the offered bids and can identify which of them belong to the winning set. These modeling assumptions are the simpliest ones, and of course can be criticized.

- The preference structure is assumed to remain stable during the auction.

The preference structure used within this framework is assumed to be stable: the $(P, I, J)$ relations do not evolve during the auction. On the other hand, one can imagine that, due to the bids' comparison, the auctioneer could further learn about his preference structure and so may want to refine it during the auction. In such context, we should consider $(P, I, J)$ relations that depend on the time, i.e. $(P(k), I(k), J(k))$ relations. In order to keep some consistence with our framework, we should impose, at least, the following restriction:

$$
P(k) \cup I(k) \subseteq P(k+1) \cup I(k+1)
$$


This constraint insures that a bid that has been "beaten" at time $k$ will remain beaten at time $k+1$. A direct corollary is that $J(k+1) \subseteq$ $J(k)$, i.e. the incomparability relation is weakened during the auction. Dynamic preference structure will be thoroughly discussed in the next chapter.

\section{- The Leap Frog method is not a multicriteria auction.}

The definition of a multicriteria auction does not encompass every auction formats considered up-to-now. The Leap Frog method, presented in the previous chapter, is based on the classic dominance relation. However it imposes a much more restrictive rule than the one considered here since every new bid must be preferred to the previous one. On the contrary, if we consider the classic dominance relation as the preference structure, our rule leads to what we have called dominance based multicriteria auctions.

\section{- Application of the auction rule: computational issue.}

The multicriteria auction's definition imposes that each new bid must not be preferred or indifferent to a bid that has already been accepted. So, at time $\mathrm{k}$, in order to be accepted, the $k^{\text {th }}$ bid $b(k)$ must be compared to the $k-1$ former bids (in the worst case). Furthermore, if we count the total number of comparisons done since the auction's beginning, we have: $\sum_{i=1}^{k}(i-1)=\frac{1}{2} \cdot k \cdot(k-1)$. This distinctive feature is due to the fact that our assumptions about the $(P, I, J)$ relations are relatively poor. A number of particular preference structures, compatible with our framework, reduce drastically this bound. For instance, if the auctioneer chooses to use a multi-attribute linear utility function, only $(k-1)$ comparisons are needed. Moreover, due to the assumption $a(P \cup I) b, b \preceq c \Rightarrow a(P \cup I) c$, the $\frac{1}{2} \cdot k \cdot(k-1)$ iterations are rarely reached; a number of dominated bids can appear during the auction and so must no be taken into account for the comparisons. In the most optimistic scenario the bids are such that $b(1) \succ b(2) \succ b(3) \ldots$ and only $(k-1)$ have to be performed.

- A first consequence of the auction rule: each new bid is influenced by the entire auction history

When the concept of bidding set has been introduced we have intuitively commented it as the set of all the bids that seller $s_{i}$ could offer. With the aforementioned auction rule, no seller $s_{i}$ is sure to be able to propose any bid $b_{i} \in \delta_{i}$ since this depends on the bids previously proposed by the other sellers: if $s_{j}$ proposes, at first, $b_{j}$ such that $b_{j}(P \cup I) b_{i}, s_{i}$ will not be allowed to later propose $b_{i}$. An example 
illustrating this phenomenon and its consequences will be discussed in section 4.5.

\section{- A second consequence of the auction rule: the competitive-} ness increases with the number of bids

A direct consequence of the auction principle is that the competitiveness increases with the number of sellers. In this specific context, the competitiveness may also increase with the number of bids. To illustrate this distinctive feature let us consider the following example: two sellers $s_{1}$ and $s_{2}$ participate to a multicriteria auction. We assume that $\xi\left(v_{1}\right) P \xi\left(v_{2}\right)$, so $s_{1}$ is the unique winning bidder. Let us consider two different scenarios. In the first scenario, we assume that $s_{2}$ directly proposes $b_{2}(1)=\xi\left(v_{2}\right)$. In response, $s_{1}$ only has to propose any bid $b_{1}$ such that $b_{1} P \xi\left(v_{2}\right)$ (such bid always exist since $\xi\left(v_{1}\right) P \xi\left(v_{2}\right)$ ). In the second scenario, at time $\mathrm{k}, s_{1}$ has already proposed $k_{1}$ bids $\left(b_{1}(1), . ., b_{1}\left(k_{1}\right)\right.$ and $s_{2}$ has proposed $k_{2}=k-k_{1}$ bids $\left(b_{2}(1), . ., b_{2}\left(k_{2}\right)\right.$. Then, let us assume that $s_{2}$ has no other choice than proposing $b_{2}\left(k_{2}+1\right)=\xi\left(v_{2}\right)$. Now, $s_{2}$ must propose a bid $b_{2}$ that simultaneously beats $\xi\left(v_{2}\right)$ and is not preferred or indifferent to the previous bids (including his own bids). Each bid present in the auction history reduces the possibilities, for a seller, to offer new bids, and so increase the competitiveness of the auction. This distinctive feature will also be illustrated on an example in section 4.5. Finally, let us notice that this effect is mainly due to the fact that the preference structure considered here is relatively poor. Consequently opportunities exist to propose bids that, for instance, do not beat all the previous bids. If the $(P, I, J)$ relations are enriched (we may for instance require that the $\mathcal{S}$ relation is transitive) such opportunities are reduced and this phenomenon disappear.

\section{- A third consequence of the auction rule: a bid acceptance does not imply a bid rejection}

In most auction contexts, when a new bid is accepted, i.e. if it satisfies the auction rules, another bid is automatically rejected. In classic English auctions, for instance, when a new bid is accepted, i.e. when the difference between the new price and the current one is greater or equal to the bid decrement, the current winning bid is rejected. In our context, this implication is not true anymore since a new bid can just be incomparable to all the bids from the winning set. In such settings the new bid will enter the winning set but no bid from the winning set will be eliminated. 


\section{- Seller's bidding behavior}

Our approach is essentially oriented towards the auctioneer's perspective; his preference structure is explicitly taken into account while the seller's are assumed to follow an idealized - basic - behavior (referred as the aggressive bidding behavior - this point will be detailed in the next section). Nevertheless, even with this simplistic position, the analyze of the present framework is far from being obvious. A number of potential extensions can be envisaged. Among them, an interesting direction for future researches is certainly the explicit consideration of the sellers' preferences.

\section{- The winning set is not always reduced to one single bid.}

Due to the potential incomparabilities between bids the winning set is, most of the time, not reduced to one single bid. As already mentioned, when the preference relation is a complete pre-order, the winning bid is univocally determined. If it is not the case, several incomparable bids might belong to the winning set. This distinctive feature may be shocking for researchers involved in auction theory since, at the end of the auction, the winning seller is not determined. On the contrary, several winning bids have been selected as 'potential' candidates and the auctioneer has still to decide which one will be chosen. This last phase is conducted for instance with one-to-one negotiations. This perspective is close to what Kersten et al. (63) calls combinations between auctions and negotiations. In this context, auctions are viewed as a first competitive step which is, then, completed with negotiations.

\section{- Why not using the kernel to select the winning bids ?}

The winning set has been defined as the set of non preferred bids from the auction history. Other definitions can, of course, be considered. Among them the notion of kernel is widely used within the multicriteria decision aid community. Nevertheless the following example illustrates why, according to us, this notion is not appropriate to the auction context.

Let us consider a multicriteria auction $M A$ with three bidders $s_{1}, s_{2}, s_{3}$ competing together. Initially, $s_{1}$ offers $b_{1}$. $s_{2}$ reacts by submitting $b_{2}$ such that $b_{2} P b_{1}$. Using the kernel notion leads to consider $b_{2}$ as being the winning bid and $b_{1}$ is beaten. Now, $s_{3}$ enters the game with a bid $b_{3}$ such that $b_{3} P b_{2}$ and $b_{3} J b_{1}$. Now applying the kernel notion leads to the set $\left\{b_{1}, b_{3}\right\}$ and $b_{1}$ reappears in the 'winning set' even it has been beaten before. Furthermore, this effect is not a consequence of $s_{1}$ strategy but due to a third party strategy. 


\section{- Cognitive complexity of the bidding process}

Even if we have assumed that the preference structure chosen by the auctioneer is public to the sellers, the bidding process, itself, can be particularly complex. It is, indeed, far different from the simple "beat the quote" rule used in classic auctions. On the contrary, complex trade-offs can be considered and the distinctive features of the $(P, I, J)$ relations have to be exploited. Confronted with these difficulties most of the sellers will not participate to the multicriteria auction unless they are supported by adequate decision tools. "Multicriteria bidding support tools" are thus of the uttermost importance in order to insure the development and the good use of these mechanisms. This statement has already been stressed by Teich (116).

\section{- Reservation levels and bid increments / decrements}

Due to the abstract nature of this chapter, practical considerations such as bid decrements (increments) and reservation levels have not been studied up to now. Their impacts on the auction results must, nevertheless, not be neglected. In a multicriteria auction context, we claim that the extension of these notions is not obvious. The question related to bid decrements will be explicitly deepened in the next chapter. On the other hand, extending the notion of reservation 'price' could appear to be straightforward: one need to define for each criteria $E^{j}$ a level $r^{j}$ over which the bids are not accepted. However, by doing so, we do not consider the multidimensional nature of the problem and so, ignore a number of different reserve configurations such as reservation constraints integrating several attributes. To illustrate this point, let us consider a procurement situation characterized by two attributes: the price $p \in[0,100]$ and the delay for delivery $t \in[0,10]$. In such context, the auctioneer may want to state that the maximum price is equal to 100 minus a penalty for every delivery days. More rigorously; $p \leq 100-5 * t$. Only bids satisfying this reservation constraint will be accepted.

On the other hand, the nature of the attributes has also to be taken into account. In classic auctions, the only attribute considered is the price. In such context, only one reservation level has to be fixed. Nobody will imagine to fix a second reservation level if the price would become too low. Nevertheless, for other attributes, two distinct reservation levels are meaningful. The delay for delivery is, for instance, such an attribute. One could, indeed, imagine that the auctioneer only accepts values in the range $[2,7]$. Over 7 days the delay is considered to be too important. Below 2 days the delay is too short. 
Consequently, the question related to reservation levels is far from being obvious and certainly constitutes a direction for future researches.

\section{- Questions related to the criteria}

Within this framework, few assumptions have been made regarding the criteria. This is, above all, due to the fact that we want to remain as general as possible. If further assumptions are needed in the subsequent developments they will be, each time, clarified.

Nevertheless, a fundamental question that is addressed in many models, such as those based on a multi-attribute linear utility function, is to wonder if the criteria are preferentially independent. In case of dependence, works based on the Choquet integral $(50 ; 72 ; 80)$, for instance, offer an elegant solution and their application to the multiattribute auction context should not lead to too many problems. In the same spirit, the following example illustrates an approach where the dependence between criteria is taken into account.

\section{Example 12 A link between multi-objects and multi-attribute auctions}

Let us consider a combinatorial auction context where items are supposed only to present synergies. As already stressed, once the auctioneer has received all the bids, he has to face the CAP (Combinatorial Auction Problem), i.e. finding the partition of items that maximizes his revenue. More formally, he has to solve the following optimization problem:

$$
\begin{array}{r}
\text { Max } b \cdot y \\
A \cdot y \leq I
\end{array}
$$

where $b$ is a vector representing the highest bids for each subset of items, $y$ is a boolean vector such that $y_{k}=1 \Leftrightarrow$ the $k^{\text {th }}$ subset belong to the optimal partition and $A$ is a boolean matrix such that $A_{i j}=1 \Leftrightarrow$ item $i$ belongs to subset $j$. The previous formulation intuitively means that the auctioneer is trying to maximizes his revenue under the constraint that a same object may not be assigned to more than one bidder. The dual form of this integer program may be written as follows:

$$
\begin{aligned}
& \operatorname{Min} x \cdot I \\
& x \cdot A \geq b
\end{aligned}
$$


where $x \in \mathbb{R}^{+(q)}$. This formulation may be interpreted as the problem an auctioneer is facing in a particular multi-attribute auction. The item sold is evaluated on q criteria noted $\left\{x^{1}, x^{2}, \ldots, x^{q}\right\}$. Due to the nature of $A$, the constraints have the form $x^{i}+x^{j}+\ldots+x^{k} \geq b_{i, j, \ldots, k}$. Let us note $\mathcal{I}=\{1,2, \ldots, q\}$. Moreover, $\forall A, B \subset \mathcal{I}: b_{A \cup B} \geq b_{A}+b_{B}$ (if some constraints do not verify this condition, they are, anyway, redundant with the other constraints). In order to interpret this, let us examine a practical example:

$$
\begin{array}{rrr}
\min \quad x^{1}+x^{2}+x^{3} & \\
\text { st : } \quad x^{1} & & \geq 3 \\
& & \geq 3 \\
& x^{2} & \geq 3 \\
& & \geq 7 \\
x^{1}+x^{2} & \\
x^{1} & +x^{3} & \geq 8
\end{array}
$$

In the spirit of goal programming approaches, the bound of the constraints denotes the desired values for the different attributes (and coalition of attributes). So imposing $x^{1} \geq 3$ leads to consider that the value $x^{1}=3$ is the ideal value for $x^{1}$. Moreover, the constraint $x^{1}+x^{2} \geq 7$ expresses the fact that the attributes $x^{1}$ and $x^{2}$ are dependent since the auctioneer is already satisfied with a sum equal to 7 instead of imposing a more restrictive bound, i.e. equal to 6 (since each attribute taken separately must be greater or equal to 3). Of course, we implicitly assume, here, that the attributes are commensurable. This formulation can thus be interpreted as a goal programming approach where the ideal's definition takes into account dependencies between attributes.

To conclude, let us point out that number of these points will be illustrated on the pedagogical example in section 4.5.

\subsection{Properties}

In this section, our attention will be focused on the properties of the winning set and the winning bids. 


\subsubsection{Properties related to the winning set}

A direct corollary of the previous definitions leads to:

Proposition 1 Let $M A$ be a multicriteria auction, we have $\forall b \in H_{M A}(k) \backslash$ $W S_{M A}(k): b \notin W S_{M A}(\tilde{k}), \forall \tilde{k} \geq k$

This property insures that a bid that has been excluded from the winning set at time $\mathrm{k}$, will never re-appear later in the winning set. As already mentioned, this property is not obvious for other possible definitions of the winning set.

Another interesting property of the winning set is that its determination only depends on the previous winning set and the last bid proposed. This is especially important for computational issues.

Proposition 2 Let $M A$ be a multicriteria auction, we have $W S_{M A}(k)=$ $N P S\left(W S_{M A}(k-1) \cup b(k)\right)$

Proof: Let $b \in H_{M A}(k)$.

- Let us assume that $b \notin N P S\left(W S_{M A}(k-1) \cup b(k)\right) \Rightarrow \exists \tilde{b} \in H_{M A}(k) \mid \tilde{b}(P \cup$ $I) b \Rightarrow b \notin W S_{M A}(k)$.

- Let us assume that $b \notin W S_{M A}(k)=N P S\left(H_{M A}(k)\right) \Rightarrow \exists \tilde{b} \in H_{M A}(k) \mid \tilde{b}(P \cup$ $I) b$. Futhermore $H_{M A}(k)=\left(H_{M A}(k-1) \backslash W S_{M A}(k-1)\right) \cup\left(W S_{M A}(k-\right.$ 1) $\cup b(k))$. If $\tilde{b} \in W S_{M A}(k-1) \cup b(k) \Rightarrow b \notin N P S\left(W S_{M A}(k-1) \cup b(k)\right)$. If $\tilde{b} \in H_{M A}(k-1) \backslash W S_{M A}(k-1)$, the auction rules imply that $b \notin$ $W S_{M A}(k-1) \cup b(k) \Rightarrow b \notin N P S\left(W S_{M A}(k-1) \cup b(k)\right)$.

\subsubsection{Characterization of the winning bids}

This subsection is devoted to the characterization of the winning bids. As described in chapter 1, a fundamental result of auction theory is that the winning bid in a 'traditional' English auction is equal to the second highest valuation. Such statement is of the uttermost importance since it facilitates 
the comparison between different auction formats. In the same spirit, the following developments help to locate, within the bidding space, where is lying the winning set of a multicriteria auction.

At first, we still need to introduce the notion of competitive set and to specify a theoretical behavior for the bidders.

Definition 16 Let $\chi_{i}=\chi\left(\xi\left(v_{i}\right)\right)=\left\{b \in \prod_{j=1}^{q} E^{j} \mid \exists b_{i} \in \delta\left(\xi\left(v_{i}\right)\right): b_{i}(P \cup I) b\right\}$ be the competitive set of $s_{i}$.

Intuitively, $\chi_{i}$ is the set of all bids that could be beaten by an offer proposed by seller $s_{i}$. A direct corollary of this definition is that $\delta_{i} \subseteq \chi_{i}$. Let us remind the reader that by 'beaten' we mean either to be explicitly preferred by another bid or being unable to propose an offer since an indifferent bid is already present in the auction history. Furthermore thanks to the restrictions imposed on the relation $\mathcal{S}$ by the mixed transitivity assumption, we have the next property:

Proposition $3 \xi\left(v_{j}\right) \in \chi_{i} \Rightarrow \forall b_{j} \in \delta_{j}: b_{j} \in \chi_{i}$.

This property insures that if an offer is preferred or indifferent to the valuation of a seller then this offer will be preferred or indifferent to any bid proposed by this seller.

Let us finally note that the definition of $\chi_{i}$ may not be reduced to $\{b \in$ $\left.\prod_{j=1}^{q} E_{j} \mid \xi\left(v_{i}\right)(P \cup I) b\right\}$ since we haven't imposed the property $a \preceq b, b(P \cup$ $I) c \Rightarrow a(P \cup I) c$ ! In other words, some bids $b_{i}$ proposed by seller $s_{i}$ are preferred of indifferent to a particular bid $\tilde{b}$ while $\xi\left(v_{i}\right) \neg(P \cup I) \tilde{b}$. Example 13 illustrates such a preference structure.

\section{Example 13 An extension of the classic dominance relation.}

Let us consider an auction characterized by 2 commensurable criteria (of which the values are supposed to be positive). The auctioneer decides to base the bids comparison on the following preference structure.

$$
b \mathcal{S} \tilde{b} \Leftrightarrow b^{i}-\tilde{b}^{i} \leq \min _{k} b^{k}, \forall i \Leftrightarrow \max _{i}\left(b^{i}-\tilde{b}^{i}\right) \leq \min _{k} b^{k}
$$

In other words, $b \mathcal{S} \tilde{b} \Leftrightarrow b^{i} \leq \tilde{b}^{i}+\tau, \forall i \in\{1,2\}$ (where the 'tolerance' parameter $\left.\tau=\min _{k} b^{k}\right)$. Obviously, this definition can be interpreted as an extension of the classic dominance relation. 
It is trivial to verify that this preference structure satisfies the mixed transitivity assumption $b_{1} \preceq b_{2} \Rightarrow b_{1} \mathcal{S} b_{2}$ since in this case $b_{1}^{i}-b_{2}^{i} \leq 0 \leq \min _{k}\left\{b^{k}\right\}$. Furthermore, if $b_{1}(P \cup I) b_{2}$ and $b_{2} \preceq b_{3}$ then $b_{1}^{i}-b_{3}^{i} \leq b_{1}^{i}-b_{2}^{i} \leq \min _{k}\left\{b_{1}^{k}\right\}$. Consequently, we have $b_{1}(P \cup I) b_{2}$.

On the other hand, let us consider the three next bids: $b_{1}=(1,3), b_{2}=$ $(4,4)$ and $b_{3}=(5,1) . b_{1} \prec b_{2} . b_{2} \mathcal{S} b_{3}$ since $b_{2}^{1}-b_{3}^{1}=4-5 \leq \min _{i}\left(b_{2}^{i}\right)=4$ and $b_{2}^{2}-b_{3}^{2}=4-1=3<4$. Unfortunately, we cannot state that $b_{1}(P \cup I) b_{3}$ since $b_{1}^{2}-b_{3}^{2}=3-1=2>1=\min _{i}\left(b_{1}^{i}\right)$.

Finally, the next assumption specifies a theoretical behavior for the bidders.

Definition 17 Aggressive Bidding Behavior: while the auction rules allow him to continue, at each time $k$, any seller $s_{i}$ offers new bids unless he has reached his valuation or he's currently in the winning set and $\forall b_{j} \in$ $W S_{M A}(k-1), j \neq i: b_{j} \notin \chi_{i}$

In other words, at each time $\mathrm{k}$, any bidder $s_{i}$ who is not in $W S_{M A}(k-1)$, is supposed to offer new bids to enter it. If a bidder $s_{i}$ is already in $W_{M A}(k-1)$, he will continue to submit new offers in order to rule out bids, proposed by other bidders, from $W_{M A}(k-1)$. Consequently, this assumption imposes a high competition level between the sellers.

Of course, weaker assumptions can be considered. One could, for instance, argue that a seller could stop offering new bids as soon as he is in the winning set. With reference to the previous assumption, this could be called the passive bidding behavior. Unfortunately, it is easy to see that, in this context, we could have the following result: $\exists b_{k}, b_{j} \in W S_{M A}$ while $\xi\left(v_{k}\right) \in \delta_{j}$. Let us consider an auction involving two bidders $s_{1}$ and $s_{2}$, such that $\xi\left(v_{1}\right) \in \delta_{2}$ and two bids $b_{1} \in \delta_{1}$ and $b_{2} \in \delta_{2}$ such that $b_{1} J b_{2}$. At a certain time $\mathrm{k}$, the winning set could be $W S_{M A}(k)=\left\{b_{1}, b_{2}\right\}$. The two bidders being in the winning set, they stop offering new bids while $\xi\left(v_{1}\right) \in \delta_{2}$. Efficiency is not respected in the sense that a bid from the winning set could be beaten by a potential bid offered by another seller.

All these statements are, of course, subject to the auction rule, i.e. the fact that a bid proposed is accepted or not, according to the auction history. This distinctive feature is of the uttermost importance since it could have deep 
consequences on the auction results as shown on the following illustrative example.

Example 14 Let us consider 3 sellers $s_{1}, s_{2}$ and $s_{3}$ competing together in a 2 criteria auction. For simplicity, $E_{1}=E_{2}=\{0,1, \ldots, 5\} . \quad \xi\left(v_{1}\right)=$ $(4,1), \xi\left(v_{2}\right)=(3,3)$ and $\xi\left(v_{3}\right)=(2,5)$. The auctioneer decides to base the bids comparison on the following preference structure:

$$
b \mathcal{S} \tilde{b} \Leftrightarrow b^{i}-\tilde{b}^{i} \leq 1(i \in\{1,2\}), b^{1}+b^{2} \leq \tilde{b}^{1}+\tilde{b}^{2}
$$

First of all, it is obvious to verify that this definition is compatible with assumption 2. Furthermore, we have $\xi\left(v_{1}\right) \mathcal{S} \xi\left(v_{2}\right)$ and $\xi\left(v_{2}\right) \mathcal{S} \xi\left(v_{3}\right)$. One could thus expect that the only winning bidder of this multicriteria auction should be $s_{1}$. Let us now imagine the following scenario. At time 1, s $s_{1}$ proposes $b_{1}(1)=\xi\left(v_{1}\right)\left(W S(1)=\left\{b_{1}(1)\right\}\right)$. Then, $s_{3}$ reacts by offering $b_{3}(1)=\xi\left(v_{3}\right)$. $A s b_{3}(1) J b_{1}(1)$ we have $W S(2)=\left\{b_{1}(1), b_{3}(1)\right\}$. Unfortunately, $s_{2}$ cannot react since, even if $b_{2}(1)=\xi\left(v_{2}\right) \mathcal{S} b_{3}(1)$, there is a bid from the auction history $b_{1}(1)$ such that $b_{1}(1) \mathcal{S} b_{2}(1)$ and consequently $b_{2}(1)=\xi\left(v_{2}\right)$ is rejected by the auction rule and the auction ends with $s_{3}$ being in the winning set.

The previous example, even if quite particular, has demonstrated how the bids history can affect the auction results. As expected, when characterizing the winning bids, this distinctive feature is particularly difficult to manage. Consequently, we will voluntary neglect it in the subsequent developments. The following results can, thus, be criticized with respect to this choice and correspond to an idealized theoretical context.

First observations lead us to note that only sellers whose valuations are not preferred or indifferent by other bids may win the auction. Final bids are thus restricted to the sub-space constituted by the bidding sets corresponding to these particular sellers. This argument is formalized in the following proposition.

Proposition 4 Let $M A$ be a multicriteria auction and let $K$ be the time of its end. Under the assumption of aggressive bidding behavior, we have:

$$
W S_{M A}(K) \subset \Omega=\bigcup_{i \in W} \delta_{i}
$$

where $W=\left\{s_{i} \in S \mid \nexists j \neq i \xi\left(v_{i}\right) \in \chi_{j}\right\}$. 


\section{Proof:}

It is easy to see that if $s_{i} \in S$ is such that $\exists j \neq i, \xi\left(v_{i}\right) \in \chi_{j}, s_{i}$ will never win the auction. Indeed, under the assumption of aggressive bidding behavior, sellers $s_{j}(\forall j \neq i)$ will compete against him until $s_{i}$ has reached his valuation.

Furthermore, as a consequence of the competition between sellers, one may exclude bids that are beaten during the auction process.

Proposition 5 Let $M A$ be a multicriteria auction and let $K$ be the time of its end. Under the assumption of aggressive bidding behavior, we have:

$$
W S_{M A}(K) \subset \Omega \backslash \Gamma
$$

where $\Gamma=\bigcup_{i, j \in S, i \neq j}\left(\delta_{i} \cap \chi_{j}\right)$

\section{Proof:}

$$
\bigcup_{i, j \in S, i \neq j}\left(\delta_{i} \cap \chi_{j}\right)=\left\{\bigcup_{i \in W} \bigcup_{j \in S, j \neq i}\left(\delta_{i} \cap \chi_{j}\right)\right\} \bigcup\left\{\bigcup_{i \in \bar{W}} \bigcup_{j \in S, j \neq i}\left(\delta_{i} \cap \chi_{j}\right)\right\}
$$

where $\bar{W}=S \backslash W$. Let us note that:

$$
\begin{aligned}
& \bigcup_{i \in W} \bigcup_{j \in S, j \neq i}\left(\delta_{i} \cap \chi_{j}\right)=\left\{\bigcup_{i \in W} \bigcup_{j \in W, j \neq i}\left(\delta_{i} \cap \chi_{j}\right)\right\} \bigcup\left\{\bigcup_{i \in W} \bigcup_{j \in \bar{W}}\left(\delta_{i} \cap \chi_{j}\right)\right\} \\
&=\left\{\bigcup_{i \in W} \bigcup_{j \in W, j \neq i}\left(\delta_{i} \cap \chi_{j}\right)\right\} \bigcup\left\{\bigcup_{j \in \bar{W}} \chi_{j} \cap\left(\bigcup_{i \in W} \delta_{i}\right)\right\} \\
&=\left\{\bigcup_{i \in W} \bigcup_{j \in W, j \neq i}\left(\delta_{i} \cap \chi_{j}\right)\right\} \bigcup\left\{\bigcup_{j \in \bar{W}} \chi_{j}\right\} \text { (because } \\
&\text { we limit ourselves to } \Omega) \\
& \bigcup_{i \in \bar{W}} \bigcup_{j \in S, j \neq i}\left(\delta_{i} \cap \chi_{j}\right)=\bigcup_{i \in \bar{W}} \delta_{i} \cap\left\{\bigcup_{j \in S, j \neq i} \chi_{j}\right\} \\
&\left.=\bigcup_{i \in \bar{W}} \delta_{i} \text { (by definition of } \bar{W}\right)
\end{aligned}
$$

Finally,

$$
\bigcup_{i, j \in S, i \neq j}\left(\delta_{i} \cap \chi_{j}\right)=\left\{\bigcup_{i, j \in W, i \neq j}\left(\delta_{i} \cap \chi_{j}\right)\right\} \bigcup\left\{\bigcup_{j \in \bar{W}} \chi_{j}\right\}
$$

As already mentioned, loosing bidders, i.e. bidders $s_{j}$ belonging to $\bar{W}$, are competing until they reach their valuation. So, any bid that may be 
preferred by, or indifferent with $b_{j}$ must be removed from the set of potential winning bids. This argument is translated by the second term of the last expression. Furthermore, winning bidders, i.e. bidders $s_{i}$ belonging to $W$ are competing together. Let $s_{i_{1}}, s_{i_{2}} \in W$. All the bids in $\delta_{i_{1}} \cap \chi_{i_{2}}$ are bids proposed by $s_{i_{1}}$ that could be preferred by or indifferent with a bid proposed by $s_{i_{2}}$. According to the aggressive bidding behavior assumption these bids must be removed from the set of potential final bids. This distinctive feature is taken into account by the first term of the last expression.

Until now, we have always assumed that each seller $s_{i}$ had only one evaluation $\xi\left(v_{i}\right)$. This choice has been made both for simplicity reasons and in order to keep the notations simple enough. However the results presented in this chapter may be directly extended to the case where each seller $s_{i}$ has $n_{i}$ different items to propose $v_{i_{1}}, v_{i_{2}}, \ldots, v_{i_{n_{i}}}$. In this case, the previous result is naturally extended as follows:

$$
\Gamma=\bigcup_{i, j \in S, i \neq j}\left\{\left[\bigcup_{l=1}^{n_{i}} \delta\left(v_{i_{l}}\right)\right] \bigcap\left[\bigcup_{l=1}^{n_{j}} \chi\left(v_{j_{l}}\right)\right]\right\}
$$

Finally it is worth noting that, under the aggressive bidding behavior assumption, winning sellers stop offering new bids as soon as they are in the winning set and that they can't exclude any other bid from the winning set by submitting new offers. One may thus exclude, from the winning set, excessive offers, i.e., offers that were not necessary to win the auction. The set of what we call excessive offers is implicitly defined as follows.

Definition 18 The set of excessive offers, noted $\Delta$, is defined as follows: let $a \in \Omega \backslash \Gamma, a \in \Delta$ if $\exists A=\left\{a_{1}, \ldots, a_{l} \mid a_{i} \in \delta(a) \backslash \Gamma\right\}$ such that $\chi(a) \cap \Gamma \subseteq$ $\bigcup_{i=1}^{l}\left(\chi\left(a_{i}\right) \cap \Gamma\right)$

In other words, a bid is said to be in $\Delta$ if it can be replaced by a finite set of offers that are strictly better from the seller point of view while they do not reduce the competitive information of the bid. One more time, let us stress that we neglect, here, the effects outlined in example 14. Of course, the previous definition is rather difficult to exploit with general preference structures. This notion will nevertheless be illustrated in section 4.7 in particular preference contexts. 
Finally these findings lead us to the following result:

Theorem 2 Let $M A$ be a multicriteria auction and let $K$ be the time of its end. Under the assumption of aggressive bidding behavior we have:

$$
W S_{M A}(K) \subset \Omega \backslash\{\Delta \cup \Gamma\}
$$

This result is a direct corollary of the previous propositions and the definition of $\Delta$.

The winning set of a multicriteria auction is, thus, constituted by mutually incomparable offers coming from $\Omega$, that, together, 'beat' all the offers included in $\Gamma$, without suffering from unnecessary improvements.

Given these results, it is obvious that the winning set, and so, the winning bids are not unique. Moreover each winning bidder may have several bids in the winning set. These distinctive features will be illustrated in the next section.

Before the forthcoming numerical analysis, a central question will retain all our attention: if different winning sets can appear during two distinct instances of the same multicriteria auction, could one of them be better from the auctioneer point of view ?

More rigorously, let $W S=\left\{b_{1}, \ldots, b_{m}\right\}$ and $\tilde{W} S=\left\{\tilde{b}_{1}, \ldots, \tilde{b}_{n}\right\}$ be two winning sets resulting from two distinct instances of the same multicriteria auction. Do we have $\exists i \in\{1, \ldots, m\}, j \in\{1, \ldots, n\} \mid b_{i} P \tilde{b}_{j}$ and $b_{k}(P \cup$ $I) \tilde{b}_{l}, \forall k \in\{1, \ldots, m\}, l \in\{1, \ldots, n\}$. As demonstrated in example 15, the answer is positive!

Example 15 Let us consider a multicriteria auction characterized by two criteria. Let us assume that $E_{1}=E_{2}=\{0,1,2, \ldots, 5\}$. The auctioneer decides to use the following preference structure:

$$
b \mathcal{S} \tilde{b} \Leftrightarrow b \preceq \tilde{b} \text { or } b+(1,-1) \preceq \tilde{b}
$$

First of all, let us verify that this relation satisfies the mixed transitivity assumption. If $a \mathcal{S} b$ two cases need to be considered. If $a \preceq b$ the condition $a \mathcal{S} b, b \preceq c \Rightarrow a \preceq b, b \preceq c \Rightarrow a \preceq c \Rightarrow a \mathcal{S} c$. On the other hand, if $a+$ $(1,-1) \preceq b$ we have $a \mathcal{S} b, b \preceq c \Rightarrow a+(1,-1) \preceq b, b \preceq c \Rightarrow a+(1,-1) \preceq c \Rightarrow$ $a \mathcal{S} c$. 


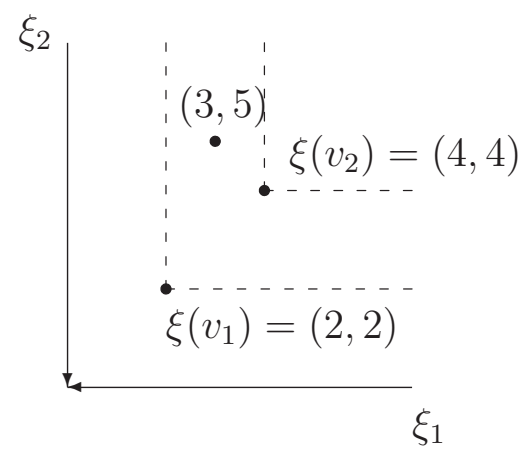

Figure 4.3: Illustration of a 2 criteria auction context with 2 distinct winning sets. $W S_{1}=\left\{b_{1}=(4,4)\right\}, W S_{2}=\left\{\tilde{b}_{1}=(3,5)\right\}$. However $\tilde{b}_{1} P b_{1}$.

Two bidders $s_{1}$ and $s_{2}$ are competing together. $\xi\left(v_{1}\right)=(2,2)$ and $\xi\left(v_{2}\right)=$ $(4,4)$. Figure 4.3 illustrates this example. In such context, it is easy to remark that the only winning bidder is $s_{1}$. One potential winning set is $W S=\left\{b_{1}=(4,4)\right\}$. Another potential winning set is $\tilde{W} S=\left\{\tilde{b}_{1}=(3,5)\right\}$. However $\tilde{b}_{1} P b_{1}$.

This distinctive feature is related to the particular auction rule and the fact that the auction outcomes depend on the auction history.

With reference to this observation, we are pushed to characterize the winning sets in terms of pareto optimality. More formally:

Definition 19 Let $M A$ be a multicriteria auction. $A$ winning set $W S=$ $\left\{b_{1}, \ldots, b_{m}\right\}$ is said to be pareto optimal if there is no other potential winning set $W \tilde{W}=\left\{\tilde{b}_{1}, \ldots, \tilde{b}_{n}\right\}$ such that $\exists i \in\{1, \ldots, m\}, j \in\{1, \ldots, n\} \mid b_{i} P \tilde{b}_{j}$ and $b_{k}(P \cup I) \tilde{b}_{l}, \forall k \in\{1, \ldots, m\}, l \in\{1, \ldots, n\}$.

Of course, it is rather difficult to characterize pareto optimal winning sets with reference to general $(P, I, J)$ relations. This notion will be illustrated in section 4.7 where particular preference structure are considered. 


\subsection{An illustrative example based on the Elec- tre I method}

In this section, we will apply the concepts and results presented up-tonow on a pedagogical example. Our aim is, of course, purely illustrative and we do not claim that the data and criteria used here below are representative.

Let us consider the case of a company that wants to subscribe mobile phone contracts for her managers. Each of the 20 managers concerned uses, on average, his mobile phone 5 hours a month. The company wants to engage a procurement auction to select which telecommunication operators will be selected.

In this context, the company is the buyer $B$. Their are three telecommunication operators on the market noted $s_{1}, s_{2}$ and $s_{3}$ who are competing to get the contract. The item being sold is a mobile phone contract supporting 20 different users. A common volume of 100 hours a month is shared by the managers at a fixed price per minute noted $p_{1}$ (on average 5 hours a month per manager). At the end of the month, if the total communication time exceeds the 100 hours, the extra telecommunication hours are charged at price per minute $p_{2}\left(\geq p_{1}\right)$. Otherwise, the remaining hours are reported to the next month.

$B$ decides to evaluate the bids on three criteria: $\xi_{1}=p_{1}, \xi_{2}=p_{2}$ and $\xi_{3}$ is the extra time (expressed in hours) offered by the operator each month (no charge) if the total communication time exceeds 100 hours. To illustrate the meaning of $\xi_{3}$ let us consider the following case. We assume here that $\xi_{3}=5$. If the total communication time is equal to 103 , the company will pay $100 \cdot p_{1}$ instead of $100 \cdot p_{1}+3 \cdot p_{2}$. If it is equal to 112 the company will pay $100 \cdot p_{1}+7 \cdot p_{2}$.

Each seller $s_{i}$ has one potential contract, noted $v_{i}$, to propose and its valuation according to these three criteria is presented in table 4.1.

Seller $s_{1}$ is the operator able to provide the lowest value for $p_{1}$. However he has the highest value of $p_{2}$ and he cannot offer any extra time. He's the best of the three operators if the company is sure that the communication package will not exceed 100 hours a month. $s_{2}$ is the best operator for $p_{2}$ and has the biggest extra time to offer. With reference to the value of these parameters, he's the best operator if the communication time exceed the package of 100 hours. Finally, operator $s_{3}$ can propose a good price for $p_{1}$ and a small extra-time but the value of $p_{2}$ remains high. The results listed 
Table 4.1: Seller's best contract evaluated according to the 3 criteria

\begin{tabular}{|l|ccc|}
\hline & $p_{1}\left(\xi_{1}\right)$ & $p_{2}\left(\xi_{2}\right)$ & extra time $\left(\xi_{3}\right)$ \\
\hline$\xi\left(v_{1}\right)$ & 0.125 & 0.25 & 0 \\
$\xi\left(v_{2}\right)$ & 0.135 & 0.15 & 20 \\
$\xi\left(v_{3}\right)$ & 0.13 & 0.2 & 10 \\
\hline
\end{tabular}

Table 4.2: Mobile phone contract procurement auction. Total cost, for each seller, evaluated on three particular scenarios.

\begin{tabular}{|c|ccc|}
\hline Tot. communication time & $s_{1}$ & $s_{2}$ & $s_{3}$ \\
\hline $100 \mathrm{~h}$ & 750 & 810 & 780 \\
$115 \mathrm{~h}$ & 975 & 810 & 840 \\
$130 \mathrm{~h}$ & 1200 & 900 & 1020 \\
\hline
\end{tabular}

in table 4.2 confirm theses statements.

We suppose here that the operators are able to propose any bid such that $b_{i} \succeq \xi\left(v_{i}\right)$. This assumption is, of course, excessive since the cost parameters depend on the value taken by the other parameters. However, for the purpose of simplicity, we will ignore this distinctive feature. Here are examples of potential bids: $b_{1}=(0.135,0.28,0) \in \delta_{1}, b_{2}=(0.15,0.15,10) \in \delta_{2}$ or $b_{3}=$ $(0.15,0.25,5) \in \delta_{3}$

$B$ decides to base the bids comparison of the ELECTRE I method (122). The parameters characterizing his preferences are fixed as follows: $w_{1}=$ $0.35, w_{2}=0.35$ and $w_{3}=0.3$ for the weights and $\phi_{1}=\phi_{2}=0.1$ and $\phi_{3}=15$ for the discordance levels (discordance happens whenever the difference is greater (respect. lower) or equal to $\phi_{j}$ ). Finally, the concordance threshold $\gamma$ is fixed to $4 / 3$. Furthermore, it is easy to verify that the Electre I method satisfies the mixed transitivity assumption. These parameters and the choice of the method is public to the sellers.

The auctioneer states that the minimum bid increment for each criteria are 0.005 for $\xi_{1}$ and $\xi_{2}$ and 5 for $\xi_{3}$. Table 4.3 illustrates one potential history of the auction.

The auction history listed in table 4.3 presents a number of distinctive features that need to be commented. First of all, let us remark that, due to 
Table 4.3: Mobile phone contract procurement auction. Application of the Electre I. Illustration of one potential auction realization.

\begin{tabular}{|c|ccccc|}
\hline Step & Bid id. & $\xi_{1}$ & $\xi_{2}$ & $\xi_{3}$ & WS \\
\hline 1 & $b_{1}(1)$ & 0.18 & 0.3 & 0 & $\left\{b_{1}(1)\right\}$ \\
2 & $b_{2}(1)$ & 0.18 & 0.28 & 0 & $\left\{b_{2}(1)\right\}$ \\
3 & $b_{3}(1)$ & 0.18 & 0.28 & 5 & $\left\{b_{3}(1)\right\}$ \\
4 & $b_{1}(2)$ & 0.17 & 0.26 & 0 & $\left\{b_{1}(2)\right\}$ \\
5 & $b_{3}(2)$ & 0.16 & 0.27 & 5 & $\left\{b_{3}(2)\right\}$ \\
6 & $b_{2}(2)$ & 0.20 & 0.25 & 10 & $\left\{b_{2}(2)\right\}$ \\
7 & $b_{3}(3)$ & 0.15 & 0.24 & 0 & $\left\{b_{3}(3)\right\}$ \\
8 & $b_{2}(3)$ & 0.20 & 0.25 & 15 & $\left\{b_{2}(3), b_{3}(3)\right\}$ \\
9 & $b_{3}(4)$ & 0.15 & 0.24 & 10 & $\left\{b_{3}(4)\right\}$ \\
10 & $b_{2}(4)$ & 0.145 & 0.23 & 0 & $\left\{b_{2}(4)\right\}$ \\
11 & $b_{3}(5)$ & 0.14 & 0.22 & 10 & $\left\{b_{3}(5)\right\}$ \\
12 & $b_{2}(5)$ & 0.15 & 0.18 & 15 & $\left\{b_{2}(5)\right\}$ \\
\hline
\end{tabular}

the particular choice of the parameters characterizing the Electre I method, a bid $b$ outranks $\tilde{b}$ as soon as $b$ is strictly better for at least two of the three attributes than $\tilde{b}$ (and of course satisfies the (strict) discordance constraint). Furthermore due to the values listed in table 4.1 , we have $\xi\left(v_{2}\right) P \xi\left(v_{1}\right)$ and $\xi\left(v_{2}\right) P \xi\left(v_{1}\right)$. Consequently, $s_{2}$ is expected to be the unique winning seller of this auction.

Let us now take a close look at the auction's history. The first three bids are basic in the sense that $b_{1}(1) \succ b_{2}(1) \succ b_{3}(1)$. Naturally, at time $3, b_{3}(1)$ is the only bid present in the winning set. Then, seller $s_{1}$ reacts by improving its latest bid $b_{1}(1)$ and propose $b_{1}(2)$. $b_{1}(2) P b_{3}(1)$ since it is strictly better than $b_{3}(1)$, for $\xi_{1}$ and $\xi_{2}$, and only worst for $\xi_{3}$. Furthermore, it respects the discordance constraints. It is easy to verify that $b_{1}(2)$ can be accepted as a new bid since it is not preferred or indifferent to a bid from $H_{M A}(3)$ (this constraint is, of course, satisfied for all subsequent bids). Similar comments justify step 5 . At time 6 , seller $s_{2}$ decides to propose $b_{2}(2)$. This is particularly interesting since it is the first time, within this auction, that a bidder does not improve one of its previous bids (in the classic dominance meaning). $b_{2}(2)$ is, indeed, strictly better than $b_{2}(1)$ for $\xi_{2}$ and $\xi_{3}$ but worst for $\xi_{1} . b_{2}(2)$ is now the only bid in the winning set. Furthermore, the nature of $b_{2}(2)$ is such that $s_{1}$ leaves the auction consequently $b_{2}(2) \mathcal{S} \xi\left(v_{1}\right)$ and so $\forall b_{1} \in \delta_{1}: b_{2}(2)(P \cup I) b_{1}$. At time $7, s_{3}$ outbids $s_{2}$. Then, at time 
Table 4.4: Mobile phone contract procurement auction. Application of the Electre I. Illustration of a second auction realization.

\begin{tabular}{|c|ccccc|}
\hline Step & Bid id. & $\xi_{1}$ & $\xi_{2}$ & $\xi_{3}$ & WS \\
\hline 1 & $b_{1}(1)$ & 0.13 & 0.26 & 0 & $\left\{b_{1}(1)\right\}$ \\
2 & $b_{3}(1)$ & 0.22 & 0.25 & 5 & $\left\{b_{3}(1)\right\}$ \\
3 & $b_{2}(1)$ & 0.18 & 0.19 & 10 & $\left\{b_{2}(1)\right\}$ \\
\hline
\end{tabular}

8, another interesting phenomenon happens: $s_{2}$ offers a bid $b_{2}(3)$ that is strictly worst than the current winning bid $b_{3}(3)$ for attributes $\xi_{1}$ and $\xi_{2}$, but is strictly better for $\xi_{3}$. Moreover, the difference on this last attribute is important enough to state that $b_{2}(3) J b_{3}(3)$ and both bids are in the winning set. $s_{2}$ and $s_{3}$ continue to compete until $s_{2}$ proposes $b_{2}(5)=(0.15,0.18,15)$. At this time $(k=12), s_{3}$ quits the auction since $b_{2}(5) \mathcal{S} \xi\left(v_{3}\right)$. Finally, $s_{2}$ wins the auction with the bid $b_{2}(5)$. This outcome is conform with the fact that $s_{2}$ is the only seller belonging to $W$.

An important point to notice is that, due to the particular auction rule, each new bid is influenced by the whole auction history. At time 6, for instance, seller $s_{2}$ could propose $\tilde{b}_{2}(2)=(0.20,0.26,10)$ instead of $b_{2}(2)=$ $(0.20,0.25,10)$ in order to beat $b_{3}(2)=(0.16,0.27,5)$ since $\tilde{b}_{2}(2) \mathcal{S} b_{3}(2)$. However $\tilde{b}_{2}(2)$ being indifferent to $b_{1}(2)$ it is not accepted as a valid new bid.

As already mentioned, the winning set is not unique. In this specific example, $s_{2}$ being the only bidder in $\Omega$, he will be the only winning seller. However, the winning bid(s), which depends on the auction history, may be different from one auction realization to the other. Table 4.4 illustrates another auction realization which ends with a different bid.

In this example, seller $s_{1}$ begins the auction with an aggressive bid (i.e. a bid that is close to its valuation). At time $2, s_{3}$ reacts by submitting $b_{3}(1)$. $b_{3}(1)$ being indifferent to $\xi\left(v_{1}\right), s_{1}$ quits the auction. Finally, $s_{2}$ proposes $b_{2}(1)\left(b_{2}(1) P b_{3}(1)\right.$ and $\left.b_{2}(1) I \xi\left(v_{3}\right)\right)$ and wins the auction. The final bid is, here, different than the winning bid in the first realization of the auction. Furthermore, we have that $(0.15,0.18,15) P(0.18,0.19,10)$ ! This is mainly due to the fact the second realization of the auction contains less bids than the first one. As already commented, the competitiveness of the auction increases with the number of bids in the auction history. Here is striking example of this effect. 
Table 4.5: Electre I based multicriteria auction- continued

\begin{tabular}{|c|ccccc|}
\hline Step & Bid id. & $\xi_{1}$ & $\xi_{2}$ & $\xi_{3}$ & WS \\
\hline 12 & $b_{2}(5)$ & 0.15 & 0.18 & 20 & $\left\{b_{2}(5)\right\}$ \\
13 & $b_{4}(1)$ & 0.135 & 0.17 & 0 & $\left\{b_{2}(5), b_{4}(1)\right\}$ \\
14 & $b_{2}(6)$ & 0.135 & 0.16 & 5 & $\left\{b_{2}(5), b_{2}(6)\right\}$ \\
\hline
\end{tabular}

Another distinctive feature is that the parameters characterizing the Electre I method have an impact on the auction's results. At time 8, for instance, seller $s_{2}$ proposes a bid $b_{2}(3)=(0.20,0.25,15)$ that is incomparable to $b_{3}(3)=(0.15,0.24,0)$. This situation is due to the discordance level related to $\xi_{3}$ that has been fixed to 15 . If the auctioneer had fixed it to 20, seller $s_{2}$ should have proposed $\tilde{b}_{2}(3)=(0.20,0.25,20)$ to obtain the same outcome. A number of authors have already cited and commented the impact of bid increments on the auction's results. Within this particular context, we note that the parameters of the method itself - in this case discordance levels - could also impact these results. Consequently, this is certainly a way for future research.

To further analyze this example, let us now imagine that a $4^{\text {th }}$ seller $s_{4}$ enters the game and $\xi\left(v_{4}\right)=(0.13,0.16,0)$. Let us also assume that $\tilde{b}_{2}(5)=(0.15,0.18,20)$ instead of $b_{2}(5)=(0.15,0.18,15)$. Table 4.5 resumes the end of the auction with this new conditions.

At time $13, s_{4}$ proposes an offer $b_{4}(1)$ that is incomparable with the current best bid $b_{2}(5) . s_{2}$ reacts by offering $b_{2}(6) P b_{4}(1) . s_{4}$ cannot outbid since $b_{2}(6)(P \cup I) \xi\left(v_{4}\right) . s_{2}$ being the only bidder in $W$, he's the only winning bidder. On the other hand, in this case, the final winning set is not reduced to a single offer but to two offers both proposed by seller $s_{2}$.

As already stressed, the previous example has only a pedagogical purpose and a number of important issues have been neglected. Among them we can cite what Teich (116) calls the bidder attributes, i.e. characteristics of the operators themselves. Is the operator reliable ? Is he a major market operator? How is the quality of his network? ... Moreover the cost structure is, of course, oversimplified since no distinction is made according to the call destination (same operator or not) and the time of the call (peak versus offpeak hours). Finally, the choice of the Electre I method has been motivated by simplicity reasons. From a practical point of view other methods could also be considered. Besides these simplifications, this example has permitted us 
to illustrate a number of distinctive features related to multicriteria auctions.

\subsection{Extensions to other auction formats}

Until now, our attention has been concentrated on English multicriteria auctions. This is mainly due to the fact that this auction format is the most popular and probably the most suited for procurement situations. Nevertheless, other kinds of auction will be discussed within this section. Yet, our aim is more to sketch potential extensions than to provide a detailed analysis of this problem. This will certainly constitute directions for future researches.

First of all, let us note that extending first price sealed bid auctions to the multicriteria framework is straightforward. It is, indeed, close to what Roy (99) calls an $\alpha$ problematic, and a wide range of methods have been developed to tackle this problem. In this section our attention will rather be focused on the Dutch and Vickrey versions of the multicriteria auction.

\subsubsection{Vickrey multicriteria auctions}

Extending Vickrey auctions to the multicriteria framework is certainly a perilous task since this kind of mechanisms is characterized by desirable theoretical properties.

Our approach is based on the principle that, in a uni-criterion context, Vickrey auctions are static mechanisms that lead to the same outcome, if the bidders submit their valuations, than the one of an English auction (at least if the assumption of private values is considered). However, as shown in section 4.4.2, the results of a multicriteria auction are not obvious to determine. Even under restrictive assumptions, we can only delimit an area $\Omega \backslash\{\Delta \cup \Gamma\}$ where the winning bids are located. Yet, we will base the extension of Vickrey auctions on this area as show here below:

1. Each seller $s_{i}$ participating to the auction submits one single sealed bid $b_{i}$. Let us note $\mathcal{B}=\left\{b_{i}, i=1, \ldots, q\right\}$ the set of offers received by the auctioneer.

2. The auctioneer $B$ collects all the bids and computes:

(a) $\tilde{\Omega}=\cup_{i \in \tilde{W}} \delta\left(b_{i}\right)$ where $\tilde{W}=\left\{s_{i} \in S \mid \nexists b_{j} \in \mathcal{B} \backslash b_{i}: b_{j} P b_{i}\right\}$ 
(b) $\tilde{\Gamma}=\cup_{i, j \in S, i \neq j}\left(\delta\left(b_{i}\right) \cap \chi\left(b_{j}\right)\right)$

(c) $\tilde{\Delta}$ the set of excessive offers based on $\tilde{\Gamma}$.

3. The auctioneer selects his preferred offer $b \mid b \in \tilde{\Omega} \backslash(\tilde{\Delta} \cup \tilde{\Gamma})$

$\tilde{W}$ denotes the set of potential winning sellers and is defined with reference to the strict preference relation $P$ (and not with reference to $\mathcal{S}=(P \cup I)$ as it was the case before). This distinctive feature has been motivated in order to insure that $\tilde{W}$ will never be empty. On the contrary, if the $\mathcal{S}$ relation was used, two sellers $s_{1}$ and $s_{2}$ participating to a Vickrey multicriteria auction, could propose respectively $b_{1}$ and $b_{2}$ such that $b_{1} \mathcal{S} b_{2}$ and $b_{2} \mathcal{S} b_{1}$. In this case, the set of winning sellers would be empty. With the aforementioned definition we have $\tilde{\Omega}=\left\{s_{1}, s_{2}\right\}$.

It is obvious that, by construction, if every seller $s_{i}$ submit $b_{i}=\xi\left(v_{i}\right)$ we have $\tilde{\Omega}=\Omega$ (at least if the winning sellers have not proposed indifferent bids), $\tilde{\Gamma}=\Gamma$ and $\tilde{\Delta}=\Delta$. Consequently the area where the winning bids are located is the same as the one estimated with multicriteria auctions. On the other hand, at step 3, we let, here, the auctioneer choose his preferred offer among all those satisfying the previous constraints. So, the results of a Vickrey multicriteria auction are expected to be at least as good as those obtained with a multicriteria auction (since in the latter case only a subset of $\Omega \backslash(\Delta \cup \Gamma)$ is highlighted at the end of the multicriteria auction). If a winning bid $b$ can be proposed by several potential winning sellers, we assume than the winning seller is randomly chosen.

Unfortunately, in this context, we cannot state that a dominant strategy for the seller is to offer $b_{i}=\xi\left(v_{i}\right)$. If $s_{i}$ increases his chance to be in $\tilde{\Omega}$ by offering a bid close to $\xi\left(v_{i}\right)$, his winning bid (if it is chosen by the auctioneer) is not completely determined by the bids from the other sellers (as it is case with a classic Vickrey auction). This distinctive feature - that happens with particular preference structures - is illustrated in the next example. Consequently, by offering bids $b_{i} \succ \xi\left(v_{i}\right)$, seller $s_{i}$ may expect to gain more than if he had proposed $b_{i}=\xi\left(v_{i}\right)$.

\section{Example 16 Illustration of Vickrey multicriteria auctions.}

Two sellers are competing in a Vickrey multicriteria auction based on the dominance relation. Both decide to submit their valuation i.e. $b_{1}=\xi\left(v_{1}\right)$ and $b_{2}=\xi\left(v_{2}\right)$. In such context $\tilde{W}=\left\{s_{1}, s_{2}\right\}$ and $\tilde{\Omega} \backslash(\tilde{\Gamma} \cup \tilde{\Delta})=\Omega \backslash(\Gamma \cup \Delta)$ which is represented on figure 4.4 by continuous lines (see section 4.7.2 for details). 


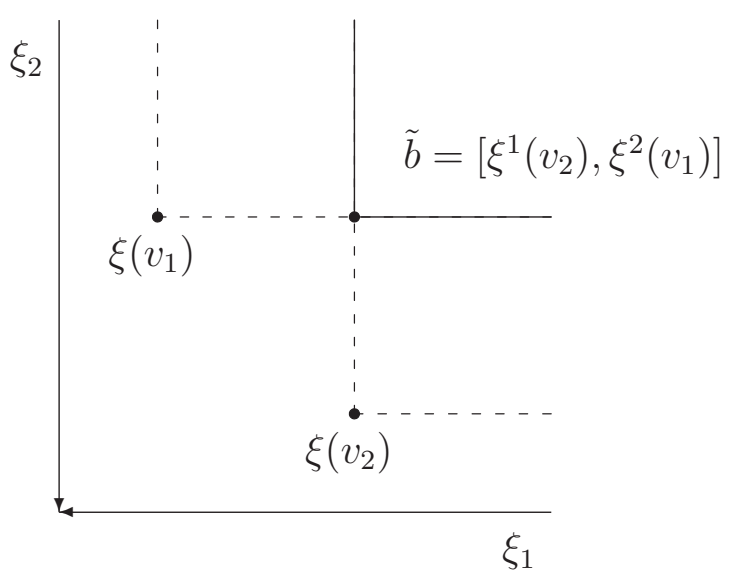

Figure 4.4: Illustration of a Vickrey multicriteria auction based on the dominance relation.

The auctioneer naturally chooses $\tilde{b}$ as the winning bid. Since this bid can be proposed by both sellers, the winning seller is randomly determined. However, the structure of $\tilde{b}=\left[\xi^{1}\left(v_{2}\right), \xi^{2}\left(v_{1}\right)\right]$ is such that it is influenced by both bids. If $s_{2}$ propose $b_{2}=\left(\xi^{1}\left(v_{2}\right), b_{2}^{2}\right)\left(b_{2}^{2} \in\left[\xi^{2}\left(v_{2}\right), \xi^{2}\left(v_{1}\right)\right]\right)$, he remains a potential winner and the winning bid $\tilde{b}$ is unaffected (nevertheless $\xi\left(v_{2}\right) \preceq b_{2}$ ).

\subsubsection{Dutch multicriteria auctions}

Even if such mechanisms are less common in procurement settings, we will propose here below an extension of Dutch auctions to the multicriteria framework.

Our approach is based on the statement that, in classic Dutch auctions, the main actor is the auctioneer: he is the only one lowering the price while the bidders can only react when they agree with its current level. Starting with these observations, we will define a Dutch multicriteria auction (DMA) in this way:

Definition 20 A Dutch Multicriteria Auction (DMA) is a succession of offers, proposed by the auctioneer, satisfying the following bidding rule: at time $k$, bids b such that $\exists \tilde{b} \in H_{D M A}(k-1): b(P \cup I) \tilde{b}$ or $\exists \tilde{b} \in A_{D M A}(k-1)$ : $\tilde{b}(P \cup I) b$ may not be proposed. $A_{D M A}$ denotes the set of offers that have already been accepted by one seller at time $k$. 
Practically, the auctioneer starts with an arbitrarily 'low' bid (since the criteria have to be minimized). Then he successively proposes bids that are not preferred or indifferent to bids previously offered, i.e. bids that are incomparable with, or at least not better than the bids from the history. At each time, any seller may accept the current bid $b$. All the bids that have already been accepted at time $\mathrm{k}$ belongs to $A_{D M A}(k)$ (In this context, $A_{D M A}$ plays the role of the winning set). Of course, the auctioneer may not propose bids b such that $\exists \tilde{b} \in A_{D M A}: \tilde{b}(P \cup I) b$ since $\tilde{b}$ has already been accepted. As a consequence of $A_{D M A}(k) \subseteq H_{D M A}(k)$ the condition $\exists \tilde{b} \in H_{D M A}(k-1): b(P \cup I) \tilde{b}$ implicitly applies to $A_{D M A}$ as well. The example 17 presented here below will illustrate how a dutch multicriteria auction takes place.

The nature of $A_{D M A}$ needs to be further discussed. With reference to the previous definition, the seller can only accepts bids proposed by the auctioneer. Once a bid has been accepted it is included in $A_{D M A}$. In this framework, we will be a little more flexible and allow sellers to accept bids that are indifferent to bids they could propose. This distinctive feature will be further explained in section 4.7.3.

Another distinctive feature related to the previous definition is the fact that the preferences of the auctioneer are explicitly token into account. On the other hand, one could, for instance, only consider the natural dominance relation in the auction rules. Since the auctioneer is the main actor of the auction and the sellers can only accept or not bids proposed by $B$ one may criticize the necessity to respect rules based on this preference structure. This point will be further analyzed in section 4.7.2 and 4.7.3.

The Dutch Multicriteria auction naturally ends when the auctioneer cannot propose any new offer, i.e. when $\forall b \in \prod_{j=1}^{q} E_{j} \exists \tilde{b} \in H_{D M A}(k): b(P \cup I) \tilde{b}$ or $\exists \tilde{b} \in A_{D M A}(k): \tilde{b}(P \cup I) b$. However the auctioneer may decide to stop the auction before, as soon as he judges he has enough bids in $A_{D M A}$. Of course in this setting, it is hard to specify in advance the time that the Dutch Multicriteria Auction will last. This is essentially due to the fact that this parameter depends on the way the auctioneer explores $\prod_{j=1}^{q} E_{j}$.

On the other hand, the fact that the auctioneer is guiding the bids evolution gives him the possibility to give greater importance to specific bid structures. This distinctive feature will be illustrated in example 17 .

Finally a last point that need to be stressed is that, contrary to classic (uni-criterion) Dutch auctions, the aforementioned definition do not impose 
Table 4.6: Mobile phone contract procurement auction. Illustration of Dutch multicriteria auctions based on the Electre I. realization.

\begin{tabular}{|c|cccc|}
\hline Step & $\xi_{1}$ & $\xi_{2}$ & $\xi_{3}$ & $A_{D M A}(k-1)$ \\
\hline 1 & 0.12 & 0.14 & 30 & - \\
2 & 0.12 & 0.14 & 15 & - \\
3 & 0.12 & 0.14 & 0 & - \\
4 & 0.12 & 0.15 & 0 & - \\
5 & 0.12 & 0.16 & 0 & - \\
6 & 0.12 & 0.17 & 0 & - \\
7 & 0.13 & 0.2 & 15 & - \\
8 & 0.13 & 0.2 & 5 & $\left\{b_{3}(1)\right\}$ \\
9 & 0.13 & 0.14 & 0 & $\left\{b_{3}(1)\right\}$ \\
10 & 0.13 & 0.16 & 0 & $\left\{b_{3}(1)\right\}$ \\
11 & 0.14 & 0.17 & 5 & $\left\{b_{3}(1), b_{2}(1)\right\}$ \\
\hline
\end{tabular}

that the attributes have to be continuously increased. In the present context, the auctioneer may decide, in one step, to impose a significant increase to one or several attributes (this point is also related to the presence of indifference thresholds - see next chapter).

\section{Example 17 Illustration of Dutch multicriteria auctions.}

In order to illustrate the use of Dutch multicriteria auctions let us consider the problem of mobile phone contracts studied in section 4.5. The auctioneer is essentially interested in two contract profiles. The first one is characterized by a low price $p_{1}$ while the two other attributes are less important. This corresponds to the ideal contract if the auctioneer is sure that the communication time will not exceed 100 hours a month. The second contract profile is oriented towards a more pessimistic scenario i.e. where the total communication time do exceed the limit imposed on $p_{1}$. In order to avoid useless illustrative bids, let us consider that the starting bid is (0.12 0.1430). Moreover due to the pedagogical nature of this example, we will - each time - voluntary increase the attributes in a subsequent way. In practice more bids are expected to appear in the auction history. Table 4.6 illustrates one realization of the auction.

As already stressed, first of all, the auctioneer wants to get the best contract with respect to $p_{1}$. He starts the auction with the bid $(0.12,0.14,30)$ and progressively degrades the values of $\xi_{2}$ and $\xi_{3}$. Steps 1 to 6 illustrates, 
first, the decrease of the extra time offered by the operator and then the increase of $p_{2}$. As consequence of the fact that no seller has reacted to the bid $(0.12,0.17,0)$, the auctioneer starts to believe that the value of $p_{1}$ is too low. So, at time 7 , he decides to increase this value to 0.13. Simultaneously, B plays on the two other attributes: he increases subsequently $p_{2}$ but on the counterpart asks for an extra time offered by the operator. Since no reaction happens, he decides to decrease the extra-time offered by the operator. Seller 3 accepts the current bid and $b_{3}(1)=(0.13,0.2,5)$ enters the set of accepted offers. With respect the valuations listed in table 4.1 one may remark that it is not the seller with the lowest value for $p_{1}$, i.e. $s_{1}$, who has won the auction. This is essentially due to the fact that $s_{1}$ is characterized by a high value for $p_{2}$ which was not considered by the auctioneer in this auction. Furthermore, the difference between $s_{1}$ and $s_{3}$ with respect to $p_{1}$ is not significant. As illustrated, each time the auctioneer has proposed offers that are not preferred of indifferent to the previous bids.

Now that a first offer has been accepted, the auctioneer wonders what would be the contract if he considers that the communication is likely to be higher than the limit of 100 hours. Consequently, he decides to be less demanding with respect to the value of $p_{1}$ (in order to maintain relatively low values for $\left.p_{2}\right)$. From now, he has to propose bids b such that $\nexists \tilde{b} \in H_{D M A}(k)$ : $b \mathcal{S} \tilde{b}$ and $b_{3}(1) \neg \mathcal{S} b$. In particular, $b$ is such that $b_{3}(1) \neg \mathcal{S} b$ and $b \neg \mathcal{S} b_{3}(1)$ $\left(b_{3}(1) \mathrm{Jb}\right)$. Steps 9,10 and 11 illustrate such bids. As already stressed, the structure of these bids is such that the emphasis is put on attributes $\xi^{2}$ and $\xi^{3}$. Finally, $B$ is pushed to propose b(11) and seller $s_{2}$ accepts it. Now, B has a bid for each of the two scenarios he had considered at first. Yet, he's not interested in a third scenario and so decides to stop the auction. Let us remind the reader that the use of Electre I has only been motivated for illustrative purpose. Other methods are, of course, more suited to manage such procurement situations.

As shown in the previous example, the rules of the Dutch multicriteria auctions are not always easy to satisfy. In this context, the development of adequate tools to support the auctioneer certainly constitutes a direction for future researches. 


\subsection{Particularizations to existing approaches}

In order to validate our approach, we will apply the previous formalism on three particular mechanisms. By doing so, uni-criterion auctions, dominance based multicriteria auctions and multi-attribute auctions will be considered.

\subsubsection{Uni-criterion auctions}

In this subsection, we will focus ourselves on classic (uni-criterion) English auctions. In this case the number of criteria $q=1, \xi\left(v_{i}\right)=\left\{\xi^{1}\left(v_{i}\right)\right\}$ is the price of $v_{i}$ and the comparison of offers is based on the $\leq$ relation. Consequently, $\chi_{i}=\delta_{i}$. The $\leq$ relation being transitive, all the information contained in the auction history at time $\mathrm{k}$ may be summarized by the current best price $\hat{b}(k)$.

According to the definition of multicriteria auctions, each new bid $b$ is such that $\nexists \tilde{b} \in H_{O}(k): \tilde{b} \leq b$, in other words; at time $k+1$ any bid $b$ is such that $b<\hat{b}(k)$. The Winning set is reduced to the current best price and consequently is unique. The aggressive bidding behavior assumption expresses the fact that any bidder continues to offer new bids until he's the winner of the auction or he has reached his valuation. It is easy to verify that the $\leq$ relation respects the constraints imposed by the mixed transitivity assumption.

Let $s_{(1)}$ and $s_{(2)}$ be the sellers with respectively the lowest and the second lowest valuation. $W=\left\{s_{(1)}\right\}$ since $\forall k \neq(1): \delta_{k} \subseteq \chi_{(1)},\left(\xi^{1}\left(v_{(1)}\right) \leq \xi^{1}\left(v_{k}\right)\right)$ while $\nexists k \mid \delta_{(1)} \subseteq \chi_{k}$ (otherwise $s_{(1)}$ would not have the lowest valuation). Consequently, $\Omega=\delta_{(1)}=\left[\xi^{1}\left(v_{(1)}\right), \infty[\right.$.

As a consequence, $\Gamma=\bigcup_{i, j \in S, i \neq j}\left(\delta_{i} \cap \chi_{j}\right)=\bigcup_{i, j \in S, i \neq j}\left(\delta_{i} \cap \delta_{j}\right)=\delta_{(2)}$ (since $\left.\delta_{i} \subset \delta_{(1)} \forall i \neq(1)\right)$. On the other hand, it is easy to show that $\Delta=$ $\left[\xi\left(v_{(1)}, \xi\left(v_{(2)}\right)-\epsilon\left[\right.\right.\right.$ since $\forall a \in\left[\xi\left(v_{(1)}, \xi\left(v_{(2)}\right)-\epsilon\left[, \exists \xi\left(v_{(2)}\right)-\epsilon\right.\right.\right.$ such that $\chi(a) \cap$ $\delta_{(2)}=\delta_{(2)}=\chi\left(\xi\left(v_{(2)}\right)-\epsilon\right) \cap \delta_{(2)}$. If $\epsilon$ is assumed to be arbitrarily low $W S=\left\{\xi\left(v_{(2)}\right\}\right.$ which is a classic theoretical result of auction theory.

It is straightforward to verify that the restrictions of Vickrey and Dutch multicriteria auctions, proposed in section 4.6, to this specific context lead to the classic versions of these mechanisms.

Let us, first, consider the case of Vickrey auctions. Once the auctioneer has collected the (uni-criterion) sealed bids, he determines $\tilde{W}$ which is, here, 
reduced to a single seller $s_{(1)}$, i.e. the one who has proposed the lowest bid. Then he computes $\tilde{\Omega} \backslash(\tilde{\Delta} \cup \tilde{\Gamma})$ which is, with respect to the previous developments, equal to the second lowest bid. Consequently, the seller with the lowest bid wins the auction but only has to 'pay' a bid equal to the second lowest bid.

Finally, the definition of Dutch multicriteria auctions leads, in this context, to a succession of offers proposed by the auctioneer such that each new offer may not be lower of equal, i.e. preferred or indifferent, to a bid that has been previously proposed. Consequently every new offer proposed by the auctioneer must be strictly greater than the previous ones. Furthermore, once a seller accepts the current offer $b$, the auctioneer stops the auction since no bid $\tilde{b}$ is such that $\tilde{b}>b$ and $b>\tilde{b}$. As a direct corollary, the set of accepted bids $A_{A D M}$ is, at the end of the Dutch auction, reduced to a single offer.

\subsubsection{Dominance based multicriteria auctions}

Let us consider now the case of what we have called dominance based multicriteria auctions (DBMA) (see chapter 3).

In this case $b_{i} \mathcal{S} b_{j} \Leftrightarrow b_{i} \preceq b_{j}$ and we have $\chi_{i}=\delta_{i}, \forall i$. The preference structure induced is such that $\preceq$ is transitive and it is straightforward to verify that $\mathcal{S}$ respects the restrictions imposed by the mixed transitivity assumption. Furthermore, it is obvious to verify that $\Omega=\bigcup_{i \in W} \delta_{i}$ where $W=\left\{s_{i} \in S \mid \nexists j \neq i: \delta_{i} \subseteq \delta_{j}\right\}$ and $\Gamma=\bigcup_{i, j \in S, i \neq j}\left(\delta_{i} \cap \delta_{j}\right)$.

Proposition 6 Let DBMA be a dominance based multicriteria auction, we have $\Delta=\Omega \backslash \Gamma$.

Proof: Let us show that $\forall a \in \Omega \backslash \Gamma, \exists A=\left\{a_{1}, \ldots, a_{l} \mid a_{i} \in \delta(a) \backslash \Gamma\right\}$ such that $\chi(a) \cap \Gamma=\bigcup_{i=1}^{l}\left(\chi\left(a_{i}\right) \cap \Gamma\right)$. In this context, $\chi(a) \cap \Gamma=\delta(a) \cap\left(\cup_{i \neq j}\left(\delta_{i} \cap\right.\right.$ $\left.\left.\delta_{j}\right)\right)$.

Let $T=\left\{t_{i j}(a) \mid t_{i j}(a)^{k}=\max \left\{\xi^{k}\left(v_{i}\right), \xi^{k}\left(v_{j}\right), a^{k}\right\}, \forall i \neq j\right\}$ and let $\tilde{T}=$ $\left\{\tilde{t}_{k}, k=1, \ldots, l\right\}$ be the subset of pareto optimum points from $T$. By construction, $a \preceq \tilde{t}_{l}, \forall l=1, \ldots, m, \delta(a) \cap\left(\cup_{i \neq j}\left(\delta_{i} \cap \delta_{j}\right)=\cup_{k=1}^{l} \delta\left(\tilde{t}_{l}\right)\right.$ and $\tilde{t}_{l} \in \Gamma$. If we define $a_{i}=\frac{a+\tilde{t}_{i}}{2}$, the aforementioned constraints are satisfied. 


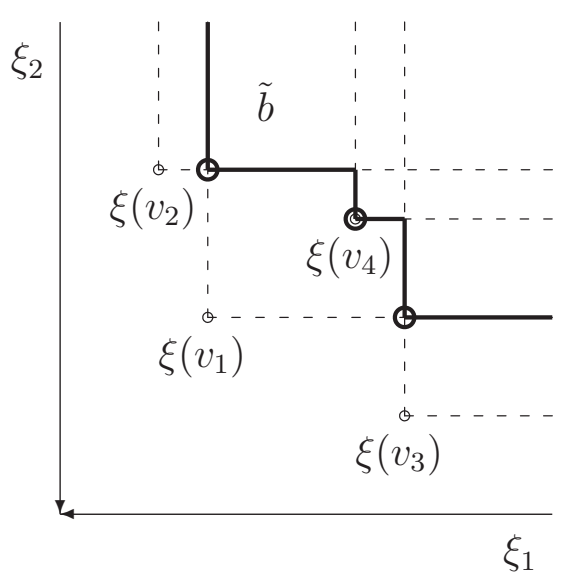

Figure 4.5: Dominance based multicriteria auctions.

Figure 4.5 illustrates the subspace $\Omega \backslash(\Delta \cap \Gamma)$ in a two criteria context. Consequently, one may expect that the dominance based multicriteria auction will end with a winning set constituted by (mutually incomparable) bids lying on the bold line. Furthermore, it is easy to verify that, in this case, the unique pareto optimal winning set is constituted by the circles lying on the bold line.

Let us now consider the application of this specific preference structure to Vickrey multicriteria auctions. One more time, once the auctioneer has collected the sealed bids, he has to determine the set of winning bidders $\tilde{W}$ and the set of potential winning bids $\tilde{\Omega} \backslash(\tilde{\Delta} \cup \tilde{\Gamma})$. With respect to this set, he finally chooses the winning bids. With reference to figure 4.5 , if the bidders reveal truthfully their valuation, we have $\Omega \backslash(\Gamma \cup \Delta)=\tilde{\Omega} \backslash(\tilde{\Delta} \cup \tilde{\Gamma})$ and one may expect that, among all the potential winning bids delimited by the bold line, the auctioneer will choose the encircled ones. However, the equivalence between winning bid and winning seller is not obvious. $\tilde{b}$, for instance, is a winning bid that could be proposed by $s_{1}$ or $s_{2}$. In such case, we plead to randomly choose the winning seller.

Finally, let us consider dominance based Dutch multicriteria auctions. In this context, the auctioneer is supposed to offer a succession of bids $b$ such that $\nexists \tilde{b} \in H_{D M A}(k): b \preceq \tilde{b}$ and $\nexists \tilde{b} \in A_{A D M}(k): \tilde{b} \preceq b$. Each time the sellers have the opportunity to accept the current offer. On the other hand, while the auctioneer respects the aforementioned rules, he is free to explore the bidding space the way he wants (and so implicitly uses his preference structure). As already stressed, these kinds of mechanisms are goal-oriented since $B$ will 
probably privilege some bidding area. Furthermore, the auctioneer may stop the auction whenever he judges he has sufficient bids in $A_{A D M}(k)$ (these distinctive features have been illustrated on example 4.6). With respect to these observations, one could conclude that Dutch multicriteria auctions could be defined only on the basis of the natural dominance relation and thus, the explicit consideration of the preference structure in the definition presented in section 4.6 .2 could be criticized. As a direct corollary, this will have the advantage that the auctioneer will not have to reveal his preference structure. Finally, one may also criticize the fact that the auctioneer will truthfully reveal a preference structure that will limit him in the exploration process (since the rules are based on it). All these critics are grounded and we do think that dominance based Dutch multicriteria auctions are certainly to be considered as a potential extension to classic Dutch auctions such as Dutch multicriteria auctions presented in section 4.6.2. Nevertheless, in the next section, we will illustrate the impact of an explicit consideration of the preference structure in Dutch multicriteria auctions.

\subsubsection{Multi-attribute auctions}

Let us consider the case of a multicriteria auction based on an additive multi-attribute linear utility function $U(b)=\sum_{j=1}^{q} \omega_{j} \cdot b^{j}(48)$ (Let us note that to be able to apply such multi-attribute linear utility function, the criteria must verify the preferential independence assumption (48)). We have $b_{i} \mathcal{S} b_{j} \Leftrightarrow U\left(b_{i}\right) \leq U\left(b_{j}\right)$. The preference structure induced is such that $\mathcal{S}$ is transitive and $J=\emptyset$. Furthermore it is easy to see that if $a \mathcal{S} b$ and $b \preceq c \Rightarrow a \mathcal{S} c$. Indeed, $a \mathcal{S} b \Rightarrow U(a) \leq U(b), b \preceq c \Rightarrow U(b) \leq U(c)$ which leads to $U(a) \leq U(b) \leq U(c) \Rightarrow a \mathcal{S} c$ (Consequently the mixed transitivity assumption is satisfied). Within this framework, sellers are competing on the values of $U(b)$. This structure is close to a classic English auction. The winner is the seller $s_{i}$ such that $U\left(\xi\left(v_{i}\right)\right) \leq U\left(\xi\left(v_{j}\right)\right), \forall j \neq i$. Let $\xi\left(v_{j}\right)$ be the second lowest valuation in terms of $U . s_{i}$ wins the auction with an offer $b_{i}$ such that $U\left(b_{i}\right)=U\left(\xi\left(v_{j}\right)\right)$. In such situation, $W=\left\{s_{i}\right\}, \Omega=\delta_{i}$ and $\chi_{k} \subseteq \chi_{i} \forall k$. Furthermore, due to the transitivity of $\mathcal{S}$, we have $\cup_{k \in S \backslash s_{i}} \chi_{k}=\chi_{j}$.

Proposition 7 Let MA be a multicriteria auction based on an additive multiattribute linear utility function, we have $\Gamma=\chi_{j}$. 
Proof:

$$
\begin{aligned}
\Gamma= & \bigcup_{l, p \in S, l \neq p}\left(\delta_{l} \cap \chi_{p}\right)=\bigcup_{l \in S}\left[\delta_{l} \cap\left(\bigcup_{p \in S, p \neq l} \chi_{p}\right)\right] \\
& =\left[\delta_{i} \cap\left(\bigcup_{p \in S, p \neq i} \chi_{p}\right)\right] \cup\left[\bigcup_{l \in S, l \neq i} \delta_{l} \cap\left(\bigcup_{p \in S, p \neq l} \chi_{p}\right)\right] \\
& =\left(\delta_{i} \cap \chi_{j}\right) \cup\left[\bigcup_{l \in S, l \neq i}\left(\delta_{l} \cap \chi_{i}\right)\right]=\left(\delta_{i} \cap \chi_{j}\right) \cup\left(\bigcup_{l \in S, l \neq i} \delta_{l}\right) \\
& =\left(\delta_{i} \cap \chi_{j}\right) \cup\left(\bigcup_{l \in S, l \neq i}\left(\delta_{l} \cap \chi_{j}\right)\right) \\
& =\bigcup_{l \in S}\left(\delta_{l} \cap \chi_{j}\right)=\chi_{j} \cap\left(\bigcup_{l \in S} \delta_{l}\right)=\chi_{j}
\end{aligned}
$$

Proposition 8 Let $M A$ be a multicriteria auction based on an additive multiattribute linear utility function, we have $\Delta=\delta_{i} \backslash \chi_{j}$.

\section{Proof:}

$\forall a \in \delta_{i} \backslash \chi_{j} \exists \tilde{a}=a+\epsilon$ such that $\chi(a) \cap \chi_{j}=\chi(\tilde{a}) \cap \chi_{j}$. Let us note that it is always possible to find such $\tilde{a} \in \delta_{i} \backslash \chi_{j}$ since $\epsilon$ is a positive vector of arbitrarily low values. Furthermore, $\left.\chi(\tilde{a}) \leq U\left(\xi\left(v_{j}\right)\right)\right)$ since if it is not the case we have: $\left.U(a) \leq U\left(\xi\left(v_{j}\right)\right)\right) \leq U(a+\epsilon), \forall \epsilon$. In other words, $U(a)=U\left(\xi\left(v_{j}\right)\right)$ which is in contradiction with the fact that $a \in \delta_{i} \backslash \chi_{j}$.

As expected, the two previous results lead us to state that $W S_{M A} \subseteq$ $\Omega \backslash(\Delta \cup \Gamma)=U\left(\xi\left(v_{j}\right)\right) \cap \delta_{i}$. Moreover, let us note, as shown in figure 4.6, that the set of potential winning bids is limited to a specific local area. By definition, all the bids lying on this local area are indifferent. Consequently, the winning set, in a multi-attribute auction, is constituted by a unique bid and every potential winning set is pareto optimal.

By using a multi-attribute utility function, we define "a virtual currency for the negotiation that expresses the overall utility of a bid to the buyer" (11). The induced multicriteria auction is thus close to a classic auction. Actually, bidders are competing on a virtual price built by means of this utility function. Extending English and First Price Sealed Bid auctions is thus straightforward. 


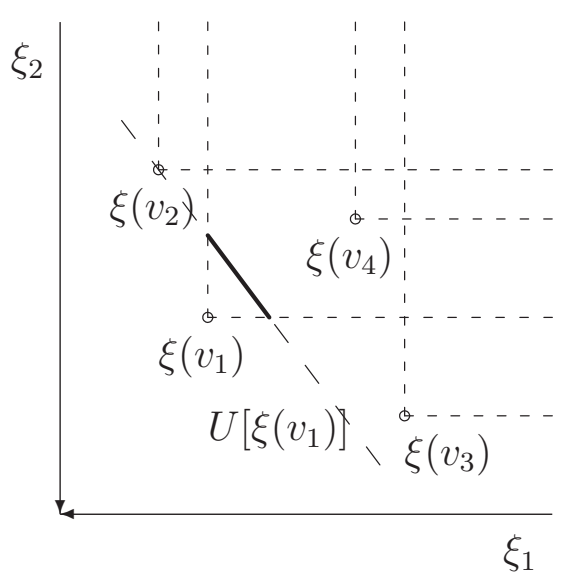

Figure 4.6: Illustration of a multi-attribute auctions.

Applying this particular preference structure to Vickrey multicriteria auctions is straightforward. $\tilde{W}$ is the bidder with the lowest bid in terms of utility and he has to propose a bid that is characterized by an utility value equal to the second lowest utility. To extend Vickrey auctions, Bichler (12) proposes to transform the gap $U\left(b_{(1)}\right)-U\left(b_{(2)}\right)$ into values of one attribute: in the case of financial derivatives trading (12), the gap is transformed into monetary value and so the winning bidder may charge a higher price for its financial product. With reference to our definition of Vickrey multicriteria auctions, the auctioneer may choose any offer in $U\left(b_{(2)} \cap \delta_{i}\right)$.

Let us, now, consider the application of linear multi-attribute utility functions to Dutch multicriteria auctions. In this case, the definition proposed in section 4.6.2 imposes that the auctioneer proposes a succession of offers such that each new bid is characterized by a higher utility than the previous ones. By incorporating the preference structure in the auction definition, we remark that the bidding space is explored in a faster way than if the classic dominance relation was used. This is a direct consequence of the fact that the set of bids that are preferred or indifferent to a bid $b$, if the classic dominance relation is used, is included in the one obtained when using a multi-attribute utility function. This distinctive feature constitutes the main advantage or incorporating the preference structure in the auction definition. Finally, it is obvious to note that the auction stops as soon as an offer $b$ has been accepted by a seller. In such a case, the auctioneer may not propose new bids since there are no bids $\tilde{b}$ such that $U(\tilde{b}) \leq U(b)$ and $U(\tilde{b})>U(b)$. A last comment that still need to be addressed is the fact that sellers may accept a bid that is indifferent to a bid they could propose. If this rule is not considered one 
could imagine that the auctioneer could propose a bid $b$ such that $\exists b_{i}$ with $U\left(b_{i}\right)=U(b)$. However, due to the fact that $b \notin \delta_{i}$, seller $s_{i}$ cannot accept the current bid. The application of this rule permits to reason on the values of the utility function and not only specific bids.

\subsection{Related questions}

This section is dedicated to two questions regarding the interest of using multicriteria (or multi-attributte) auctions. First of all, we will motivate the use of multicriteria auctions with respect to the classic request for quote alternative. Then the question related to integrated versus ex-post bids selection will be addressed.

\subsubsection{Multicriteria auctions versus RFQ}

When pleading for the use of auction mechanisms that supports multidimensional bids, a natural question is to compare them to classic Request For Quote approaches (RFQ).

The main difference between these two approaches lies in the preference revelation. In a RFQ context, the buyer has to reveal (at least partially) his preferences in order to "guide" the sellers in their submission. This phase is performed before the bidding process and so is independent from the bids submitted.

In multicriteria auctions, one can imagine that the buyer first defines (weak) preferences that he will refine then, during the auction, based on the bids he will receive. This distinctive feature has already been stressed in section 4.3.4 and will be further analyzed in the next chapter.

The important point to emphasize on is that, in an auction context, a number of different preference structures can lead to choose the same bidder as the winning seller. This comment is illustrated on figure 4.7. In this case, a linear multi-attribute utility function is used to represent the auctioneer's preferences. $b_{1}$ is selected to be the winning bid (the continuous line representing the set of iso-utility points related to the true preferences of the auctioneer). However, any iso-utility curve belonging to the arbitrary cone defined by the dashed lines could have led to the same conclusion. Consequently, even if the auctioneer does not fully reveal his preferences, the 


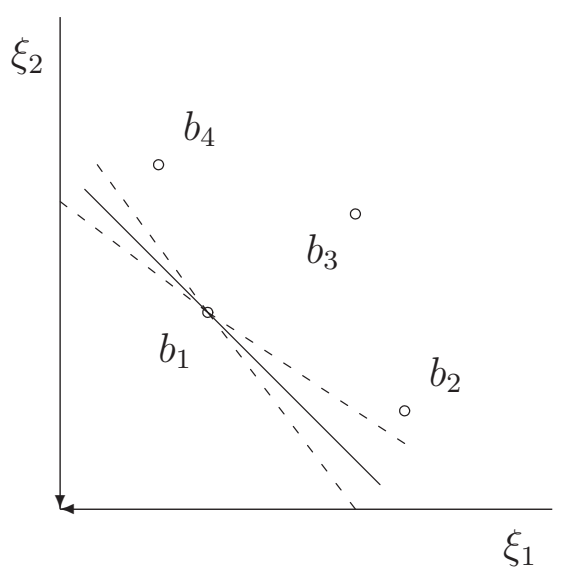

Figure 4.7: Multi-attribute auctions: different set of weights can lead to select the same winning bid.

conclusion is the same.

This statement leads to wonder how much the auctioneer has to reveal to achieve the same selection. This question is at the origin of the forthcoming empirical tests.

Of course, for simplicity reasons, we have considered here a classic linear multi-attribute utility function to define the preferences. The empirical tests have been conducted according to algorithm 1 in a 2 attributes context.

Intuitively this algorithm functions as follows. Let us assume that $p$ sellers are participating to the auction. Firstly the parameters characterizing the multi-attribute auction are randomly chosen according to a uniform distribution: the set of weights $\tilde{w}$ defining the linear multi-attribute utility function and the set of evaluations $\xi(v)$ characterizing the sellers. Once the auction context is established, the winning seller $s_{k}$ is determined; he's the bidder with lowest valuation in terms of utility. Moreover, the utility score of the winning bid $b_{k}$ is computed. As already explained, this is equal to the second lowest multi-attribute utility score. This value will later serve as a benchmark for the analysis. From now on, we will focus on the preference revelation needed to select the same bidder as the winner. Let $W$ be the set of weights leading to select $s_{k}$ as the winning bidder:

$$
W=\left\{\begin{array}{l}
0<w<1 \\
w \cdot \xi^{1}\left(v_{k}\right)+(1-w) \cdot \xi^{2}\left(v_{k}\right) \leq w \cdot \xi^{1}\left(v_{i}\right)+(1-w) \cdot \xi^{2}\left(v_{i}\right), \forall i \neq k
\end{array}\right.
$$


Intuitively the second constraint imposes that the weights are such that the utility score assigned to the evaluation of $s_{k}$ are lower or equal than the score assigned to all the other evaluations. Consequently, $s_{k}$ remains the winning bidder.

Of course, the size of $W$ gives an indication of the preference revelation needed to maintain $s_{k}$ as the winning bidder. Let $\underline{w}=\min \{W\}$ and $\bar{w}=$ $\max \{W\}$. The percentage of preferences revealed will be given by $1-(\bar{w}-\underline{w})$.

An important point to emphasize on is that this statistic corresponds to an optimistic scenario: the set $W$ is estimated directly with reference to the evaluations of the bidders and does not take into account the auction history. In reality, the auctioneer is expected to reveal more about his preferences since he will have to decide between bids offered during the auction (the number of comparisons is likely to be higher than the one considered here before and their nature is likely to be more restrictive). So, the results presented here after have to be considered as an optimistic bound.

Finally, we will compute the utility score of the winning bid in the auction context $\{\xi(v), W\}$. This value will then be compared to $U_{\tilde{w}}\left(b_{k}\right)$. First of all, as a consequence of $W$ definition and under the aggressive bidding behavior assumption it is easy to remark that the winning bid will always be equal to $\xi\left(v_{k}\right)$. Otherwise bids that could be beaten by $\xi\left(v_{k}\right)$ could lie in the winning set. Consequently, we will consider here another bidding behavior (referred as the moderate bidding behavior): a bidder stops offering new bids as soon as his valuation do not permit him to beat (for all instances of the weights $w \in W$ ) a bid that has been proposed before. The set of bids $D_{k}$ proposed by $s_{k}$ that satisfy this constraint is given by:

$$
D_{k}=\left\{b \in \delta_{k} \mid w \cdot b \leq w \cdot \xi\left(v_{j}\right) \forall j \neq k, w \in W\right\}
$$

To conclude, the (true) utility of the winning bid (Max $\left.\tilde{w} \cdot b, b \in D_{k}\right)$ is estimated and related to utility first computed.

These tests have been conducted for a number of sellers that ranges from 2 to 100 . Each time, 1000 tests have been run and the average results have been computed.

Figure 4.8 illustrates the mean preference revelation when the number of sellers varies from 2 to 100 . As expected the mean preference revelation increases with the number of sellers since more information is needed to discriminate them. This effect if particularly pronounced between 2 and 20 


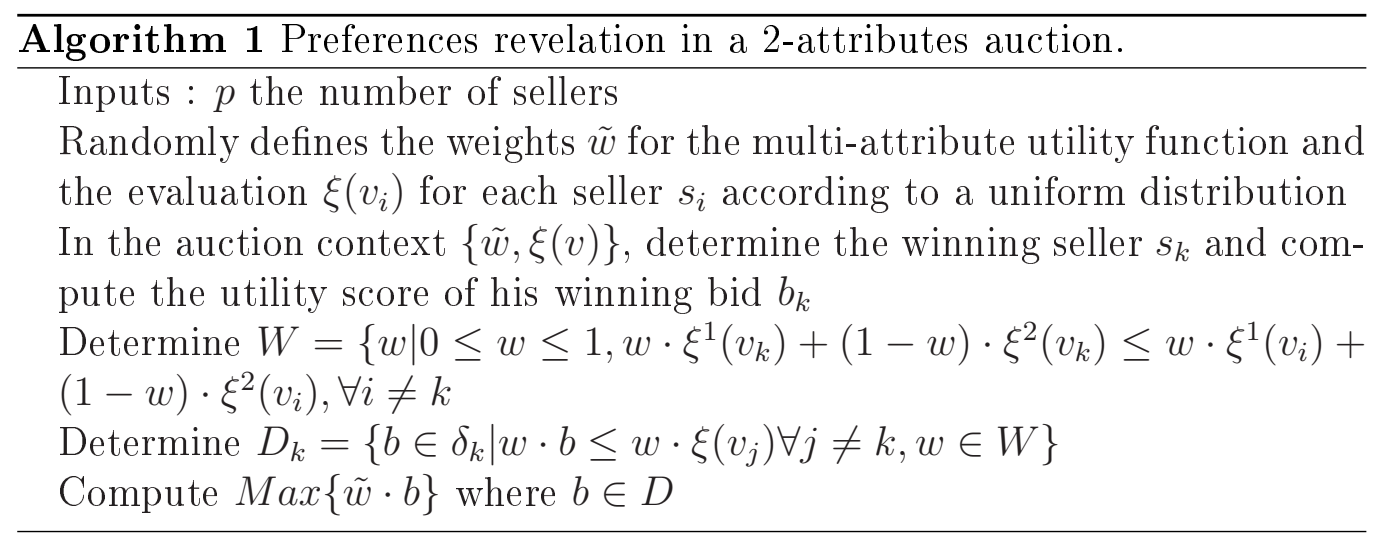

sellers. After this limit, it remains around 0.4.

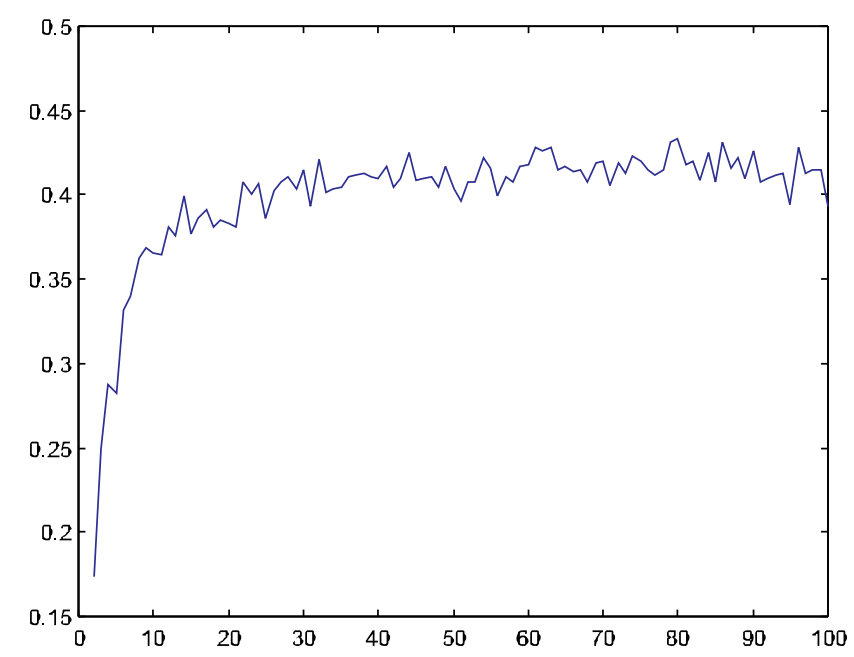

Figure 4.8: Mean percentage of preferences revealed when the number of sellers varies from 2 to 100 .

Of course, even if $s_{k}$ remains the winning seller for any instance of the weights $w \in W$, the nature of the winning bid is affected by the degree of preference revelation. If the auctioneer fully reveals his preferences, i.e., if he reveals $\tilde{w}$, the winning bid is an offer from $\delta_{k}$ of which the utility is equal to the second lowest valuation utility score. On the other hand, if the auctioneer does not reveal more than $W$, as already noticed, the winning bid is equal to $\xi\left(v_{k}\right)$. Figure 4.9 illustrates the mean utilities obtained for the first and second preferred bids and mean utilities obtained under the moderate bidding behavior. As expected the mean utilities decrease with 
the number of bidders. On the other hand, figure 4.10 presents the evolution of the utility gap rate (the difference between the moderate bidding score and the first score divided by the difference between the second and the first score).

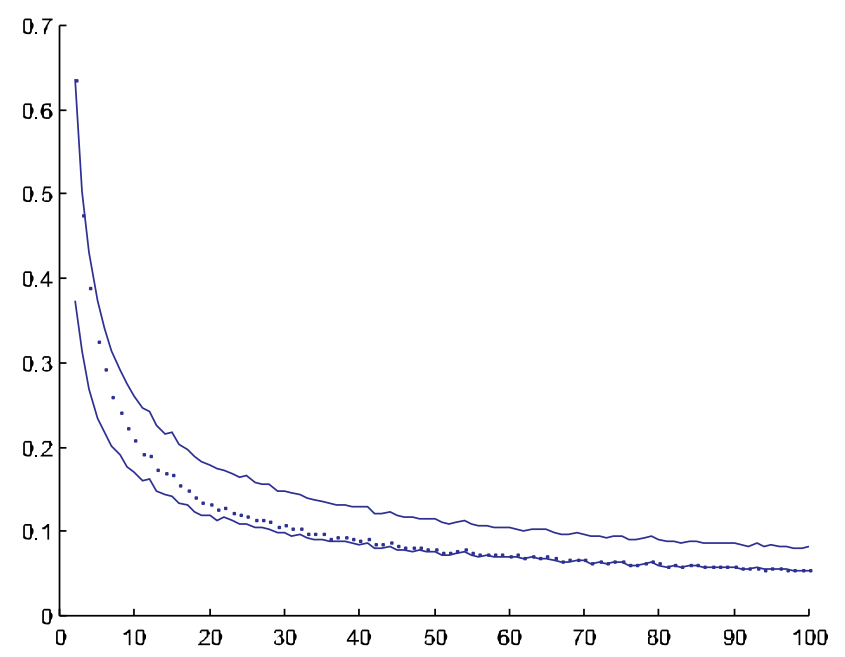

Figure 4.9: Mean utilities for first and second preferred bids (continuous lines). The dots represents mean utilities under the moderate bidding behavior.

Figure 4.8 exhibits an interesting effect: even if the preference revelation increases with a number of bidders varying from 2 to 20 - which is confirmed by the intuition - it seems, nevertheless, to remain stable around the value 0.4 for greater values of this parameter. This distinctive feature can be explained on the basis of figure 4.11. In this example, we consider a fixed utility function $U(b)=\frac{1}{2} \cdot b^{1}+\frac{1}{2} \cdot b^{2}$. Let us assume that the point $b=(u, u)$ is the winning bid. Consequently, we implicitly assume that the probability to have a bid in the triangle $\{(0,2 \cdot u),(0,0),(2 \cdot u, 0)\}, 2 \cdot k^{2}$,is negligible. Let us remark that only bids present in the area (1) and (2) will force the auctioneer to further reveal his preferences (since the other bids are simply dominated by $b$ ). In what follows we will focus ourselves to the area (1) (the same arguments applies in the case of area (2)). In this context, $w \in[1 / 21]$. In this case the probability that a bid appears in the triangle $\left\{\left(0, \frac{u}{1-w}\right),(0,2 \cdot u),(u, u)\right\}$ is equal to $2 \cdot u^{2} \cdot\left\{\frac{2 \cdot w-1}{2-2 \cdot w}\right\}$. Consequently, one may state that the auctioneer has to reveal enough preference information in order to make the aforementioned probability small enough. In other words: 


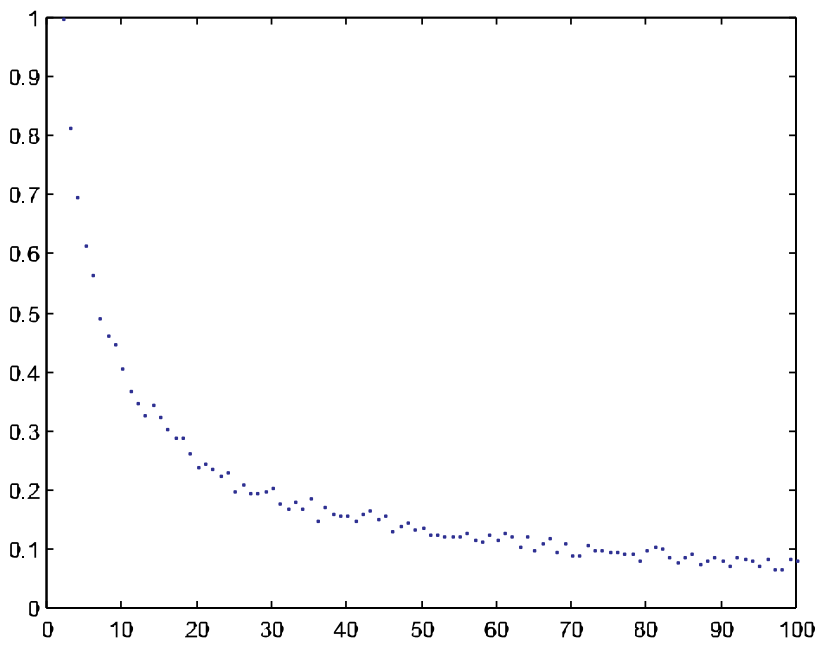

Figure 4.10: Evolution of the utility gap rate: difference between the moderate bidding score and the first score divided by the difference between the second and the first score when the number of sellers varies from 2 to 100 .

$$
\frac{1}{2} \cdot \frac{2 \cdot w-1}{1-w} \leq 1 \Leftrightarrow \leq \frac{3}{4}
$$

When applying the same argument to area (2) we get $w \geq \frac{1}{4}$. Consequently we have $\frac{1}{4} \leq w \leq \frac{3}{4}$ and the preference revelation is $\approx 0.5$. Of course these arguments are based on idealized symmetric example. Nevertheless, they permit to better understand this bound.

\subsubsection{Ex-post versus integrated bids selection}

A second question related to the use of multicriteria auctions is about the difference between ex-post and integrated bids selection.

In the present framework, the preference structure is used during the auction for the bids selection. This will be referred as the integrated bids selection. By doing so, the auctioneer has to reveal (at least partially) his preferences in order to guide the bidders. On the other hand, one may imagine a situation where the bids comparison is only based on the natural dominance relation. Once the auction is finished, the buyer selects, with respect to his preference structure, the winning bid(s) among the offers present 


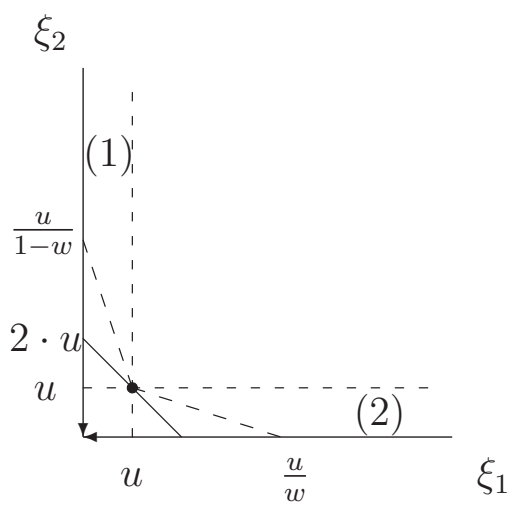

Figure 4.11: Preference revelation scheme

in the winning set (in this case, the auctioneer does not have to reveal his preferences at all). Consequently, this scenario will be referred as the ex post bids selection.

As illustrated on figure 4.12, it is easy to understand that the integrated bids selection will lead to better winning bids (from the auctioneer's point of view) than the ex post bids selection. The setting is the same as the one of figure 4.5 or 4.6 . The bold continuous line corresponding to $\underline{U}^{\text {ex post }}$ denotes potential winning bids (i.e. bids proposed by $s_{1}$ of which the utility is equal to $U(\tilde{b}))$ in the most optimistic scenario i.e. if $\tilde{b}$ is proposed during the dominance based multicriteria auction preceding the bids selection. On the other hand, the bold line corresponding to $\left.U\left(\xi\left(v_{2}\right)\right)\right)$ denotes potential winning bids in case of integrated bids selection. As illustrated, the latter case leads to a lower utility than the first one. This intuitive result is confirmed by the following proposition:

Proposition 9 Let $M A$ be a multicriteria auction and DBMA its associated dominance based auction. Every bid that is eliminated during DBMA is also eliminated during MA. More formally, $\Gamma^{\prec} \subseteq \Gamma^{(P, I, J)}$.

The proof of this proposition is a direct corollary of the mixed transitivity assumption.

In this section we propose to compare these two scenarios. For simplicity, we assume, in the following tests, that the preference structure of the auctioneer is represented by a linear multi-attribute utility function noted $U$. 


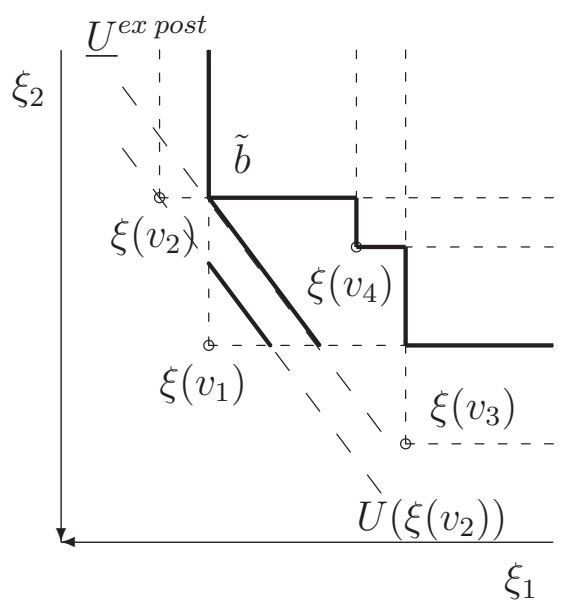

Figure 4.12: Integrated versus ex post bids selection in a 2-attributes auction.

As already explained, the utility associated to the winning bid, in the case of integrated bids selection $U_{I B S}$ is lower or equal to the one achieved in the case of ex post bid selection $U_{E P B S}$. The goal of the following empirical tests is to evaluate the gain in terms of utility $U_{E P B S}-U_{I B S}$ with reference to the number of bidders and the number of attributes associated to the auction.

These empirical tests have been run for a number of attributes varying from 2 to 5 and a number of bidders varying from 2 to 50 . Each time 100 random tests have been computed. Algorithm 2 outlines the scheme followed for these experiments. Let us note that, one more time, a random uniform $[0,1]$ distribution has been used to generate the random set of weights characterizing the multi-attribute utility function and the evaluations of the bidders. Moreover, one more time, let us stress the fact that the results associated to the ex-post scenario are optimistic since we have selected the lowest utility among all potential outcomes of the dominance based auction. These results must be considered as a lower bound for the utility.

Concerning the results showed on figure 4.13, several comments can be done. First of all, let us note that the evolution of the utility scores, in the integrated bids selection scenario, is coherent with the intuition: they decrease when the number of sellers increases and, for a same number of sellers, they decrease when the number of attributes increases. However, when comparing them to the results associated to the "ex post scenario" utilities, a particular phenomenon appears: the ratio $\bar{U}_{e}^{(1)} / \bar{U}_{i}^{(2)}$ remains more or less stable for each setting of the attributes. These ratios are, respectively, (on average) equal to $110 \%, 115 \%, 118 \%$ and $120 \%$ when the number of attributes evolve from 

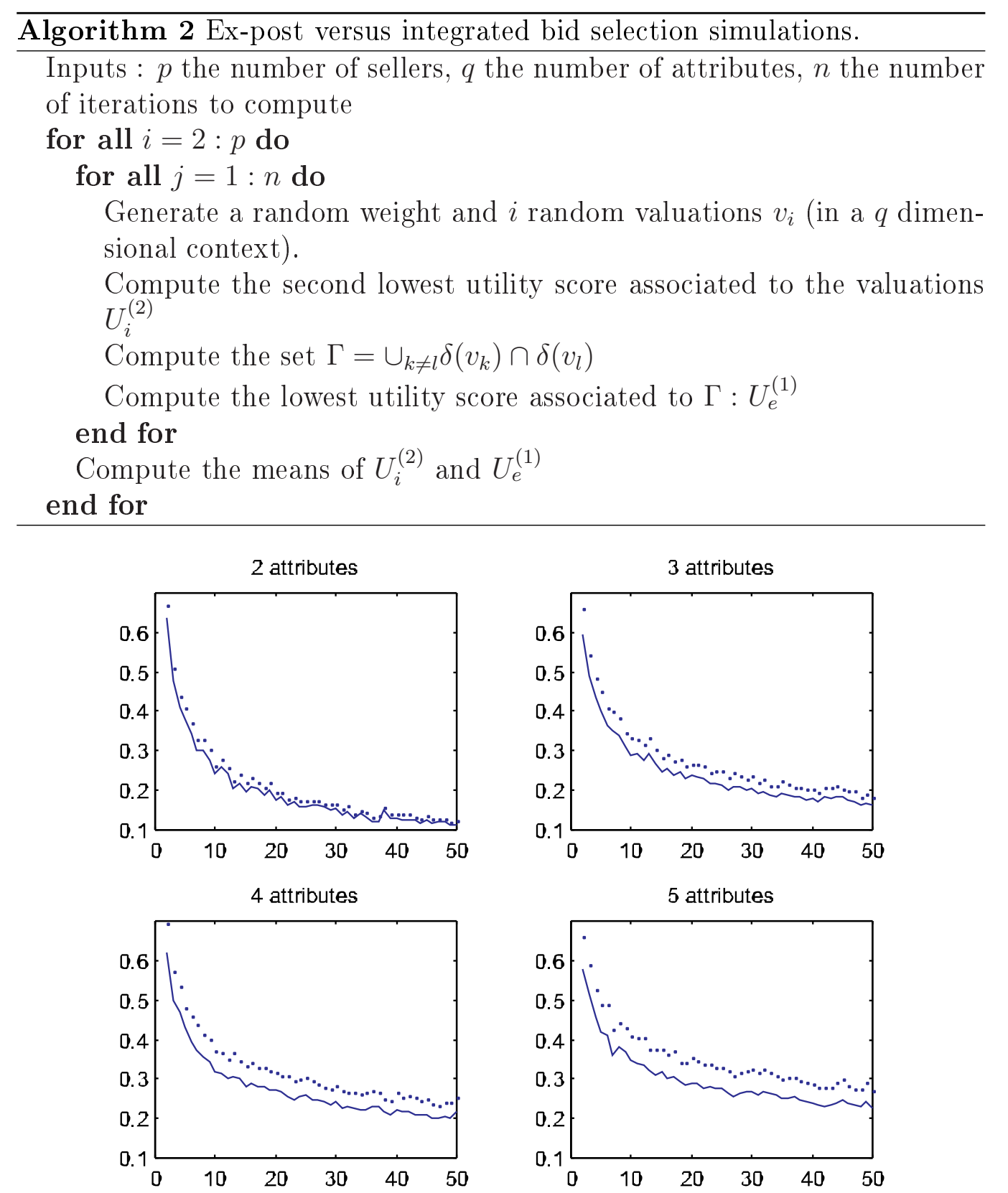

Figure 4.13: Ex-post versus integrated bids selection. Evolution of the mean (winning) utility in these two scenarios when the number of bidders range from 2 to 50 and the number of attributes varies from 2 to 5 .

2 to 5.

In order to explain why these ratios remain more or less stable when the 
number of bidders ranges from 2 to 50 we will analyze the illustrative example given on figure 4.14. We consider here a 2 attributes context. At first, 10 bids have been randomly drawn using a bi-uniform distribution $[0,1] \times$ $[0,1]$. For simplicity, the weights characterizing the multi-attribute linear utility function are equal to $w_{1}=w_{2}=\frac{1}{2}$. The two bold continuous line represents the second lowest utility in the integrated bid selection scenario $U_{2}$ and the lowest utility in the ex post bid selection scenario $U_{(1)}^{\text {expost }}$. Now, let us consider the points that are lying in the dashed square: these five points are distributed according to a uniform distribution $[0, s] \times[0, s]$. This context is similar to the one previously studied up to an homothetic tranformation of ratio $s$. In other words, if we apply the inverse transformation to the coordinates of each of these points, i.e. an homothetic transformation of ratio $\frac{1}{s}$, we will obtain a potential realization for 5 bids (i.e. in the square $\left[\begin{array}{ll}01\end{array}\right] \times\left[\begin{array}{ll}0 & 1]\end{array}\right]$. Of course, the levels of the utilities are also affected by this transformation. Nevertheless, the ratio remain the same.

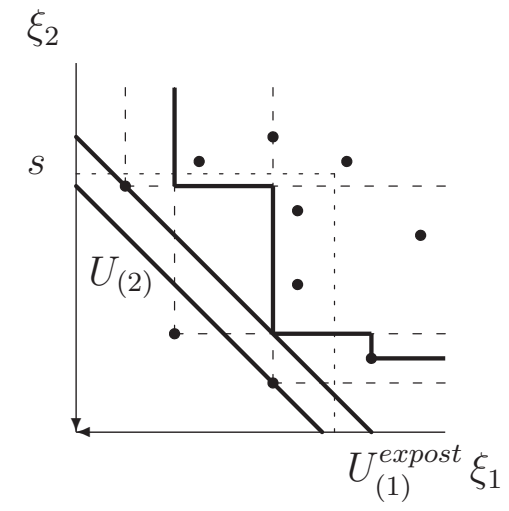

Figure 4.14: Ex-post versus integrated bid selection. The ratio $U_{(1)}^{\text {expost }} / U_{(2)}$ remains stable if we consider all the bids or only the bids lying in the dashed square.

On the other hand, the fact that these ratios increase with the number of attributes is intuitive. Indeed, if we have a close look to the utility calculated in the ex-post scenario, we have:

$$
U_{(1)}^{\text {expost }}=\min _{v_{l} \neq v_{k}} \sum_{j=1}^{q} w_{j} \cdot \max \left\{\xi^{j}\left(v_{k}\right), \xi^{j}\left(v_{l}\right)\right\}
$$

Consequently, when the number of attributes increases from $q$ to $q+$ 1 the term $\max \left\{\xi^{q+1}\left(v_{k}\right), \xi^{q+1}\left(v_{l}\right)\right\}$ is taken into account in the (ex-post) 
utility calculation. If only two sellers $s_{1}$ and $s_{2}$ participate to the auction the term $\xi^{q+1}\left(v_{1}\right)$ (respectively $\left.\xi^{q+1}\left(v_{2}\right)\right)$ is integrated in $U^{\text {integrated }}\left(\xi\left(v_{1}\right)\right)$ (respectively $U^{\text {integrated }}\left(\xi\left(v_{2}\right)\right)$ ). On the contrary, in the ex-post scenario, the term $\max \left\{\xi^{q+1}\left(v_{k}\right), \xi^{q+1}\left(v_{l}\right)\right\}$ is added to utility. In this way, the utility associated to the ex-post bids selection is expected to increase faster than the one associated to the integrated bids selection when the number of attributes increase.

Finally, with respect to the previous results, one may conclude that the ex-post bids selection do not lead to outcomes that are much worst than those obtained with the integrated bids selection (while no preference revelation is needed).However, this statement can be criticized since, as already explained, we have considered, in this analysis, optimist results for the dominance based multicriteria auction. Furthermore, the number of bids present in the winning set associated to $D B M A$ is likely to be much bigger than in the integrated bid selection.

\subsection{Conclusion}

In the past few years, different approaches have been proposed to deal with auction mechanisms that support multidimensional bids. Each contribution is referred to an underlying specific model to deal with the multicriteria nature of the problem.

Inspired by the multicriteria decision aid works, we have decided, in this chapter, to study the field at a more abstract level i.e. by considering a sufficiently general preference structure $(P, I, J)$ to manage the bids comparisons. This idea has led us to study potential incomparability relations between bids. This distinctive feature has been motivated and constitutes the main originality of our approach.

Within this context, we have been led to formalize the notions related to the idea of multicriteria auction and to discuss its related distinctive features. The properties of the winning set and the winning bids have also deeply retained our attention. Before considering particularizations of our framework to existing approaches such as dominance based multicriteria auctions, multi-attribute auctions or simply classic uni-criterion English auctions, we have illustrated these concepts on a pedagogical example based on the Electre I model. Moreover extensions of multicriteria auctions to Vickrey and Dutch multicriteria auctions have been sketched. Finally general questions 
regarding the use of multicriteria auctions have been treated.

Of course, the analysis performed in the present chapter rely on a variety of particular choices and assumptions. Most of them can be criticized (see, for instance, section 4.3.4) and the potential extensions of our work resulting from weaker assumptions and / or more general choices constitute as many directions for future researches. Nevertheless, among them, we can remind the reader of few ones that seem to us to be crucial:

- The explicit consideration of the sellers'preferences is certainly a fruitful way to explore. In the present work, we have, mainly for simplicity reasons, limited ourselves to a naive bidding behavior.

- The question of information revelation and its impact on the aforementioned results is another question that should receive more attention.

- The development of adequate Bidding support tools is intrinsic to the proper use of multicriteria auctions (due to the cognitive complexity of the bidding process).

- A detailed analysis of the extensions proposed for Dutch and Vickrey multicriteria auctions should be further explored.

To conclude, this chapter constitutes, first of all, a theoretical approach to multi-attribute auctions (in the general meaning of this term), performed with the typical tools of the multicriteria decision aid community. In this way a bridge is set between these two fields. 


\section{Chapter 5}

\section{Butterfly auctions}

\subsection{Introduction and motivations}

This chapter is, first of all, dedicated to the presentation and analysis of a particular multicriteria auction model. Due to its particular appearance, this will be referred to as the butterfly model. By extension, butterfly auctions are multicriteria auctions based on a butterfly model.

Mainly two motivations have pushed us to develop this new approach: the management of incomparability between multi-dimensional bids and the dynamic nature of the auctioneer's preference structure.

The management of potential incomparability between multi-dimensional bids has already been motivated in the previous chapter (at least with respect to an abstract $(P, I, J)$ preference structure). Moreover, this distinctive feature will lead us to consider new auction contexts such as auctions composed of different bidding niches. These notions will be formalized and empirically tested in the next chapter. The model presented in the next sections has been developed in order to be able to manage such situations.

On the other hand, as already stressed in the previous chapter, the preference structure of the auctioneer is likely to evolve during the auction. This point is related to the fact that, by comparing bids received during the auction, the buyer will probably learn about his preferences. Consequently, he may want to have the opportunity to refine his preference structure during the auction. This characteristic will be thoroughly discussed within this chapter. 
Finally, a number of questions related to the practical use of the butterfly model will retain our attention. Among others, the notion of bid increment (decrement) will be studied and related to the notion of indifference threshold which is a widespread concept in the multicriteria decision aid community. Furthermore, the problem of 'multicriteria bidding support tools' will be considered.

The present chapter is organized as follows: the next section is dedicated to the presentation of the model itself. The way the preference degrees per criterion are built will be detailed as well as their aggregation. Then, an outranking relation $\mathcal{S}$, incorporating discordance levels, will be introduced. To conclude this section, a number of distinctive features of the butterfly model will be discussed in subsection 5.2.4. In the third section, we will present some of its theoretical properties. Then related questions will be considered in section 5.4. In this way, we will tackle problems related to the preference elicitation, to bid decrements, to bidding support tool as well as some robustness considerations. In order to illustrate all these developments a pedagogical example will be described in section 5.5. Finally, a conclusion will summarize our work.

To conclude, let us stress that this chapter is inspired by the paper "Butterfly auctions: clustering the bidding space" (37).

\subsection{The model}

The model presented in this section is inspired from both the Promethee and the Electre methodologies. It is based on pairwise comparisons between multi-dimensional bids and allows (constrained) preferences tuning during the auction process.

The next subsections will detail the building of the outranking relation $\mathcal{S}(k)=(P(k) \cup I(k))$ that will lead us to state that a bid $b$ is at least as good as another bid $\tilde{b}$ at time $\mathrm{k}: b \mathcal{S}(k) \tilde{b}$. One more time, let us stress the presence of the parameter $k$ which indicates that the relation $\mathcal{S}(k)$ is likely to be refined during the auction. Finally, as already stressed in the previous chapter, let us insist on the fact that this evolution may not be arbitrary. On the contrary, we will impose, at least, that $\mathcal{S}(k) \subseteq \mathcal{S}(k+1)$. Furthermore, additional conditions imposed on $S(k+1)$ (see section 5.2.3.1) will insure that a bid that has been 'beaten' at time $k$ will remain 'beaten' until the auction's end. 
As it is done in the Promethee method, we will base our model on the differences between the two bids for each criterion. These values will then be transformed into preference degrees (per criterion) to, finally, be aggregated using a weighted sum. In this way, we will obtain a "global" measure to evaluate if the bid $b$ is at least as good as $\tilde{b}$. Additionally, as it is done in the Electre methods, we will consider discordance levels which will act as veto thresholds in the comparisons.

However, unlike what is done in the Promethee or Electre methods, we will not restrict ourselves to fixed parameters. Consequently, we will not consider, here, a unique instance of the weights in the aggregation rule but rather a (constrained) set of weights that are compatible with the auctioneer preferences. Moreover, both the discordance levels and this set of weights are likely to evolve during the auction. These points constitute the main distinctive features of our approach.

\subsubsection{Differences and preference degrees per criterion}

As already stressed, we will base the pairwise comparisons between the bids on their differences on each criterion. These rough values will constitute the basis of the model proposed. More formally, the difference between bids $b$ and $\tilde{b}$ on criterion $\mathrm{j}$ is defined as follows:

$$
d_{j}(b, \tilde{b})=\tilde{b}^{j}-b^{j}
$$

Under the assumption that all the criteria have to be minimized, a value $d_{j}(b, \tilde{b}) \geq 0$ indicates that $b$ is not worse than $\tilde{b}$ on criterion $\mathrm{j}$.

If the values $d_{j}(b, \tilde{b})$ will help us to figure out if a specific bid $b$ is at least as good as $\tilde{b}$, they cannot directly be used since their interpretation is not obvious! As it is commonly done in the multicriteria decision aid community, we will first transform these rough data into preference degrees. This phase will be performed using a general linear preference function (see figure 5.1). Two parameters are characterizing this transformation:

- an indifference threshold, noted $q_{j}$, below which the difference $d_{j}(b, \tilde{b})$ is so small than the two bids are considered to be indifferent on criterion $j$.

- a preference threshold $p_{j}$ under which the difference $d_{j}(b, \tilde{b})$ is big 
enough to state that $b$ is, definitely, better than $\tilde{b}$ on criterion $j$.

When the difference $d_{j}(b, \tilde{b}) \in\left[q_{j}, p_{j}\right]$ a linear interpolation is performed (see figure 5.1). The parameters $q_{j}$ and $p_{j}$ are fixed by the auctioneer before the auction and we assume that they remain unchanged.

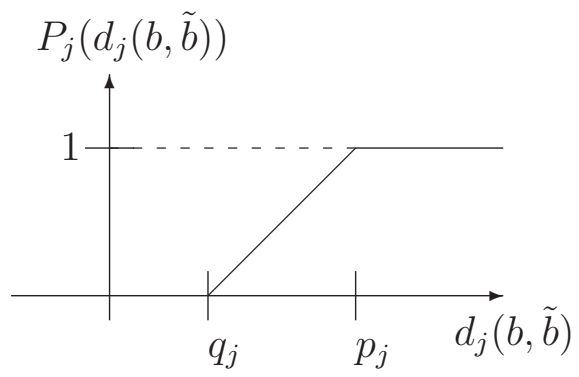

Figure 5.1: Linear preference function transforming differences between two bids according to a specific criterion into preference degrees for this criterion.

Once this step has been performed, we get, for each criterion, preference degrees between $b$ and $\tilde{b}$ (that are independent from the unity of the considered criterion).

\subsubsection{Preference degrees aggregation}

Once the preference degrees per criterion have been evaluated, one needs to aggregate them in order to get a global measure to evaluate if $b$ is at least as good as $\tilde{b}$. In accordance with the Promethee method, we have chosen to aggregate them using a classic weighted sum:

$$
P_{w}(b, \tilde{b})=\sum_{j=1}^{q} w_{j} \cdot P_{j}\left(d_{j}(b, \tilde{b})\right)
$$

where $w=\left\{w_{1}, w_{2}, \ldots, w_{q}\right\}$ represent weights compatible with the auctioneer's preferences. 
However, unlike the Promethee and Electre method, we do not assume here that these parameters are fixed by the auctioneer prior to the auction. On the contrary, we will rather assume that they belong to a subset of admissible weights, noted $A W(k)$, that is likely to evolve during the auction. When considering $P_{w}(b, \tilde{b})$, the subscript $w$ is of the uttermost importance since it emphasizes the fact that the aggregated preference is rarely equal to a fixed value but rather belongs to a set of potential values.

Intuitively, $A W(k)$ represents a set of weights compatible with the auctioneer's preferences. The index $k$ indicates that this set will evolve during the auction due to constraints added by the buyer as a consequence of the learning process. More formally, $A W(k)=\left\{\left(w_{1}, \ldots, w_{q}\right)\right\}$ such that:

1. $w_{l}>0, l=1, . ., q$

2. $\sum_{l=1}^{q} w_{l}=1$

3. $a_{i 1} \cdot w_{1}+\ldots+a_{i q} \cdot w_{q} \leq c_{i}, i=1, . ., m_{k}$

4. $\sum_{l=1}^{q} w_{l} \cdot\left(P_{l}\left(b_{i}, b_{j}\right)-P_{l}\left(b_{j}, b_{i}\right)\right) \geq 0, \forall b_{i}, b_{j} \mid b_{i} \mathcal{S}(k) b_{j}$

The first two constraints considered here above are natural, well-known restrictions for weights used within this context. The third constraint represents direct restrictions imposed by the buyer. An example of such a constraint could be $w_{1}(k)-2 \cdot w_{2}(k) \geq 0$; at time $\mathrm{k}$, the weight associated to criterion 1 is at least twice more important than the weight associated to criterion 2. Let us note that at time $\mathrm{k}$, there might be $m_{k}$ different "direct" constraints imposed on the weights. Finally the last constraint takes into account non trivial comparisons imposed by the buyer; for any pair $\left(b_{i}, b_{j}\right)$ if the auctioneer states, at time $\mathrm{k}$, that $b_{i}$ is at least as good as $b_{j}$, i.e. $b_{i} \mathcal{S}(k) b_{j}$, (while it was not the case before) the admissible weights are updated such that $P_{w}\left(b_{i}, b_{j}\right) \geq P_{w}\left(b_{j}, b_{i}\right), \forall w \in A W(k)$. Furthermore, once a constraint has been considered at time $k$, it will be considered for any time $\tilde{k} \geq k$, such that $A W(\tilde{k}) \subseteq A W(k)$. This expresses the fact that the auctioneer is refining his preferences during the auction. No backward move is allowed.

\subsubsection{The outranking relation $\mathcal{S}$}

In accordance with the Electre I method, we will choose to restrict the comparisons to a limited neighborhood, either explicitly or implicitly defined 
by the auctioneer. Let $\phi_{l}$ define the comparability window for each criterion. Intuitively, $\phi_{l}$ represents a value (related to criterion $l$ ) below which the difference between $b$ and $\tilde{b}$ is too high (in favor of $\tilde{b}$ ) to state that $b$ is at least as good as $\tilde{b}$ whatever the values on the other criteria. More formally, $\phi_{l}(k)$ is defined as follows $(\forall l=1, \ldots, q)$ :

$$
\begin{gathered}
\operatorname{Min} \phi_{l}(k) \\
\left\{\begin{array}{l}
\phi_{l}(k) \geq 0 \\
\lambda_{l}(k) \leq \phi_{l}(k) \\
\phi_{l}(k-1) \leq \lambda_{l}(k) \\
d_{l}\left(b_{i}, b_{j}\right) \geq-\phi_{l}(k), \forall b_{i}, b_{j} \mid b_{i} S(k) b_{j}
\end{array}\right.
\end{gathered}
$$

As requested, it is natural to restrict the thresholds to be positive. The constraint $\lambda_{l}(k) \leq \phi_{l}(k)$ expresses the fact that at each time $\mathrm{k}$, the parameter $\phi_{l}(k)$ must be greater than or equal to a bound $\lambda_{l}(k)$ that the auctioneer may choose to fix himself. If $\lambda_{l}(k)$ is not fixed by the auctioneer it is anyway greater than or equal to $\phi_{l}(k-1)$. This expresses the fact that the "comparability window" must not be reduced during the auction process, i.e. if some pairs of bids were judged to be comparable at some time $\mathrm{k}$, then they will remain comparable at any time $\tilde{k} \geq k$. In order to remain consistent with the parameters introduced in section 5.2.1, we will assume $\phi_{l}(0)=q_{l}$. Finally the last constraint takes into account additional information provided by the auctioneer, i.e., if the auctioneer states that $b_{i}$ and $b_{j}$ are comparable then the thresholds $\phi_{l}(k)$ are updated to satisfy this constraint.

With these definitions, we will say that $b \mathcal{S}(k) \tilde{b} \Leftrightarrow d_{l}(\tilde{b}, b) \geq-\phi_{l}(k), \forall l=$ $1, \ldots, q$ and $P_{w}(b, \tilde{b}) \geq P_{w}(\tilde{b}, b), \forall w \in A W(k)$. In other words, bid $b$ will be considered as good as $\tilde{b}$ iff the two bids are comparable and if the preference degree of $b$ over $\tilde{b}$ is greater than or equal to the preference degree of $\tilde{b}$ over $b$ for any instance of the admissible weights. More practically, $P_{w}(b, \tilde{b}) \geq$ $P_{w}(\tilde{b}, b), \forall w \in A W(k) \Leftrightarrow T(b, \tilde{b}) \geq 0$, where $T(b, \tilde{b})=\min w \cdot\left(P_{w}(b, \tilde{b})-\right.$ $\left.P_{w}(\tilde{b}, b)\right), w \in A W(k)$.

A 2-dimensional graphic interpretation of the previous parameters is given in figure 5.2. Due to its particular form, $\mathcal{S}(k)$ will be called a butterfly at time k. For simplicity reasons, we only consider points located in the subspace $[-1,1] \times[-1,1]$. Both the preference functions are assumed to be characterized by the parameters $q_{1}=q_{2}=0$ and $p_{1}=p_{2}=1$. By doing so, $P_{j}\left(d_{j}(b, \tilde{b})\right)=\max \left(0, d_{j}(b, \tilde{b})\right)$ and consequently we are able to represent on the same graph the set of admissible weights (defined on $P_{j}$ ) and the $\phi_{j}$ 


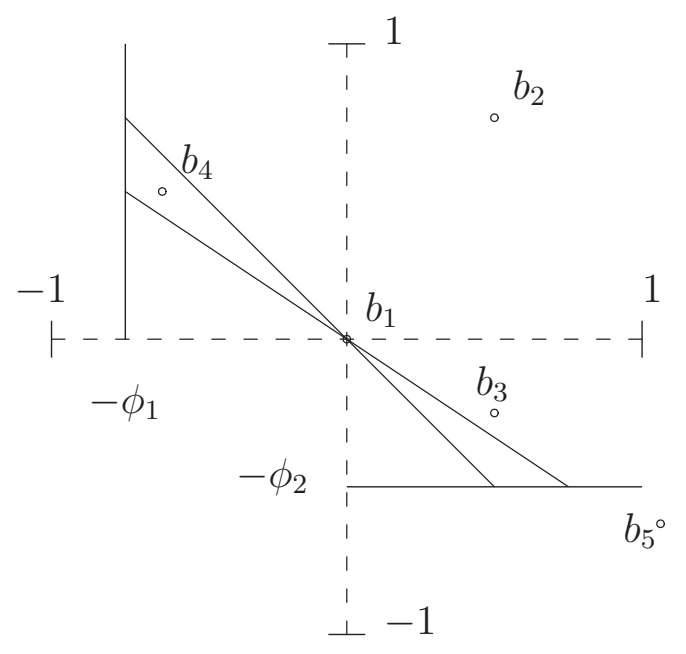

Figure 5.2: Illustration of a 2-dimensional butterfly model.

thresholds (defined on $d_{j}$ ). For simplicity reasons, these assumptions will be implicitly considered in the remaining figures.

The interpretation of figure 5.2 leads to state that $b_{1} \mathcal{S} b_{2}$ since $b_{1} \preceq$ $b_{2}$. Moreover $b_{1} \mathcal{S} b_{3}$ since $b_{3}$ lies in the comparability window of $b_{1}$ and $P_{w}\left(b_{1}, b_{3}\right) \geq P_{w}\left(b_{3}, b_{1}\right), \forall w \in A W$. On the other hand, $b_{1} \neg \mathcal{S} b_{4}$ since $\exists w, \tilde{w} \in$ $A W$ such that $P_{w}\left(b_{1}, b_{4}\right)>P_{w}\left(b_{4}, b_{1}\right)$ while $P_{\tilde{w}}\left(b_{1}, b_{4}\right)<P_{\tilde{w}}\left(b_{4}, b_{1}\right)$. Finally, $b_{1} \neg \mathcal{S} b_{5}$ since the value $b_{5}^{2}$ is much better than $b_{1}^{2}$ - in other words $b_{5}$ does not lie in the comparability window of $b_{1}$.

As stated before, it is important to stress the fact that admissible weights and comparability thresholds may be dynamically fitted during the auction process. These adjustments may be based on information provided by the auctioneer himself or deduced by the potential comparisons of submitted offers. Such a potential evolution is illustrated in figure 5.3.

\subsubsection{Constraints consistency for the set of admissible weights}

The set of admissible weights is either explicitly or implicitly defined (see subsection 5.2.2). When the auctioneer adds constraints in order to refine his preferences one may insure that no contradiction appears. If contradictory constraints are added to $A W(k)$, the set of admissible weights would be empty and no more comparisons could be computed. However, the following intuitive result permits to relativize this statement. 


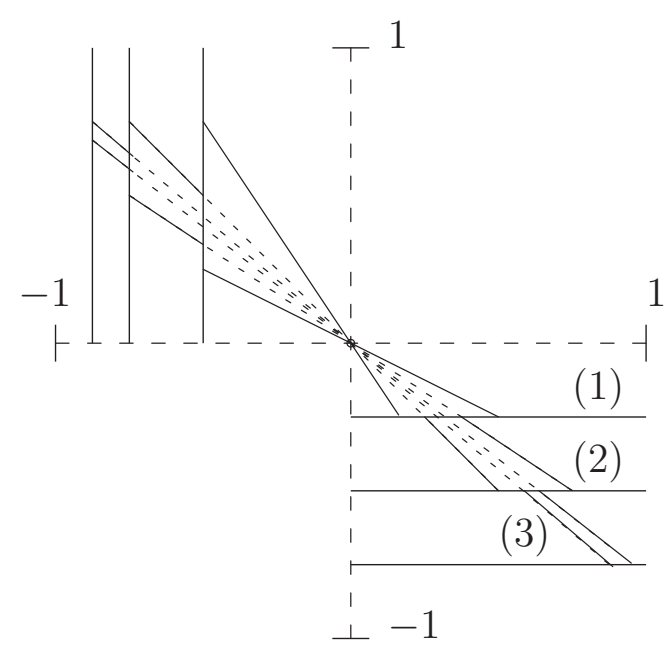

Figure 5.3: Potential evolution of a 2-dimensional Butterfly: the comparability window is progressively extended while the set of admissible weights is refined.

Proposition 10 At time $k$, if the only new constraints accepted are those induced by bids comparisons between offers from $W S_{M A}(k)$, no contradictory constraint will be added to $A W(k)$.

This result is intuitive since bids belonging to the winning set at time $k$ are, by definition, incomparable. In other words, the preference structure at time $k$ is not rich enough to decide between them.

More formally, let $b, \tilde{b} \in W S_{M A}(k)$ such that $d_{j}(b, \tilde{b}) \geq-\phi_{l}(k), \forall l=$ $1, \ldots, q$. If the auctioneer decides to state that $b \mathcal{S}(k) \tilde{b}$, the constraint $\sum_{l=1}^{q} w_{l}$. $\left(P_{l}(b, \tilde{b})-P_{l}(\tilde{b}, b)\right) \geq 0$ is added to $A W(k)$. Let us note the resulting set of admissible weights $A \tilde{W}(k)$. If $A \tilde{W}(k)$ was empty, a direct corollary would be:

$$
A W(k) \subseteq\left\{\begin{array}{l}
\sum_{l=1}^{q} w_{l}=1 \\
w_{l}>0, l=1, . ., q \\
\sum_{l=1}^{q} w_{l} \cdot\left(P_{l}(\tilde{b}, b)-P_{l}(b, \tilde{b})\right) \geq 0
\end{array}\right.
$$

Consequently $\tilde{b} \mathcal{S}(k) b$ and $b$ would not be in the winning set at time $k$.

Of course the previous argument is only valid if the constraints are added one by one to $A W(k)$ since, one constraint imposed on the pair $(b, \tilde{b})$ can have effects on other bids from the winning set. 
For the same reasons, it is easy to see that no contradiction will appear during the update of the parameters $\phi_{l}(k)$ while the constraints only concerns bids from the winning set.

Consequently, only comparisons between bids from $W S_{M A}(k)$ will be allowed, in practice, to refine the preference structure.

\subsubsection{Discussion}

The model presented in the previous subsections exhibits a number of distinctive features that need to be discussed. Among them, we can cite the following points:

\section{- Some considerations about incomparability situations.}

Two bids are judged to be incomparable iff $b \neg \mathcal{S} \tilde{b}$ and $\tilde{b} \neg \mathcal{S} b$. With respect to the model introduced in the previous subsections, the assertion $b \neg \mathcal{S} \tilde{b}$ can be the consequence of two (not necessarily exclusive) situations.

First of all, there might exist an index $j$ such that $\tilde{b}^{j}-b^{j}<-\phi_{j}$. In this way, $b \neg \mathcal{S} \tilde{b}$ since, for at least one criterion, $\tilde{b}$ is much better than $b$. As a consequence of the fact that the auctioneer may directly increase the values of the parameters $\phi_{j}$, these kinds of situations are easy to manage explicitly.

The second situation that leads to state $b \neg \mathcal{S} \tilde{b}$ is $\exists w, \tilde{w} \in A W$ such that $P_{w}(a, b)>P_{w}(b, a)$ and $P_{\tilde{w}}(b, a)>P_{\tilde{w}}(a, b)$. In this case, the assertion $b \neg \mathcal{S} \tilde{b}$ is stated since the auctioneer's preferences are not precise enough; two weights instances, compatible with the current auctioneer's preferences, lead to conflicting conclusions. Such situations will become less common whenever the auctioneer learns about his preferences and consequently reduces the uncertainty related to $A W$.

\section{- Dynamic versus fixed parameters.}

Our model is characterized by a number of parameters. Among them, we can distinguish those that are fixed before the auction and those that are likely to evolve during the bids submission.

- Fixed parameters: $q_{j}, p_{j} \forall j=1, . ., q$. These parameters, related to the intra-criterion information, are assumed to be fixed at the auction setup and to remain stable afterwards. By doing so, we assume that the auctioneer perfectly knows how to interpret the 
differences on each criterion and that the impact of the learning process (resulting from the bids comparison) on these parameters is marginal. Of course this point of view can be criticized and extensions of the present model to dynamic versions of $q_{j}$ and $p_{j}$ may be considered. However, for simplicity reasons, we will restrict ourselves to the aforementioned assumption.

- Dynamic parameters: $\phi_{j}, w_{j} \forall j=1, . ., q$. First of all, the parameters related to the inter-criteria information (i.e. the weights) are likely to be tuned during the auction. As already stressed, this is related to the fact that the auctioneer will probably learn about his preferences during the auction as a consequence of the bids comparisons. Furthermore, this distinctive feature is more than welcome since the parameters related to the inter-criteria information are certainly those that are the most delicate to fix a priori. Finally, the discordance levels $\phi_{j}$ may be progressively increased during auction. This is related to a caution approach. At first the discordance levels are assumed to be quite low, leading to a number of discordance events and consequently a number of incomparability relations. Then, with respect to these situations, the auctioneer may decide to increase the discordance levels, leading to a model that is more compensatory.

\section{- A model inspired by both the Promethee and the Electre methods}

Subsections 5.2.1 and 5.2.2 are deeply inspired by the Promethee methodology. The building of preference degrees is, indeed, very similar to what is done in (19), which is, by the way, similar to the preference modeling performed in the Electre III method (at least up to a linear transformation). Nevertheless, our approach differs by the fact that, during the preference aggregation step, we do not restrict ourselves to a specific instance of the weights but, on the contrary, we consider a whole set of admissible weights.

Another similarity with the Electre I method is the use of discordance levels per criterion noted $\phi_{j}$. Let us note that, in the context of Electre I, the use of such parameters may be criticized since their boolean nature affects the results sensitivity. However, in the present context, this phenomenon is reduced since the $\phi_{j}$ may be adapted during the auction.

\section{- Events affecting the winning set}

In most auction contexts, only new bids can affect the winning set. In 
the present framework, the possibility, for the auctioneer, to update his preferences during the auction constitutes another particular event that may affect the winning set too. This consitutes a distinctive feature of our model.

A side effect of such a property is that two bids being in the auction history at time $k$ could become indifferent as a consequence of the preferences tuning. So, indifferent bids can appear during the auction while it was not the case in the theoretical framework presented in the previous chapter.

This will, nevertheless, not affect the winning set since its definition is based on the strict preference relation. If the winning set is constituted at time $k$ by two bids $b$ and $\tilde{b}$ and if the preference tuning leads to $b \mathcal{S}(k) \tilde{b}$ and $\tilde{b} \mathcal{S}(k) b$ both bids remains in the $W S(k)$ and are stated to be indifferent. If, on the contrary, the $W S$ definition was based on the $\mathcal{S}$ relation, it would have become empty.

\section{- The butterfly model is not transitive}

As in a number of multicriteria models, the butterfly model does not fulfill the transitivity property. This distinctive feature is essentially due to the use of the comparability window. To illustrate this effect, let us consider the following particular two dimensional butterfly model: $\phi_{1}=\frac{3}{2}, \phi_{2}=0, w_{1}=w_{2}=\frac{1}{2}$ (no uncertainty on the weights). With these settings it is easy to verify that $(3,1) \mathcal{S}(2,3),(2,3) \mathcal{S}(1,5)$ but $(3,1) \neg \mathcal{S}(1,5)$.

\section{- The butterfly model is not acyclic}

As illustrated in example 18, the use of indifference and preference $\left(q_{j}, p_{j}\right)$ thresholds leads to a relation $\mathcal{S}$ that is not acyclic. Nevertheless, due to particular auction rule, no bid cycle will appear in practice. Finally, as shown in section 5.3, when $q_{j}=0$ and $p_{j}$ is big enough, the relation $\mathcal{S}$ is acyclic.

Example 18 Let us consider the three following bids: $b_{1}=(2,3), b_{2}=$ $(4,2)$ and $b_{3}=(6,1)$. In this context, we will assume $\phi_{j}=\infty, j=1,2$. $d_{1}\left(b_{1}, b_{2}\right)=2, d_{2}\left(b_{1}, b_{2}\right)=-1, d_{1}\left(b_{2}, b_{3}\right)=2, d_{2}\left(b_{2}, b_{3}\right)=-1, d_{1}\left(b_{3}, b_{1}\right)=$ -4 and $d_{2}\left(b_{3}, b_{1}\right)=2$.

At first, we will assume $w_{1}=\frac{1}{4}, w_{2}=\frac{3}{4}, q_{j}=1$ and $p_{j}=3, j=1,2$. It is easy to verify that $P_{w}\left(b_{1}, b_{2}\right)=\frac{1}{8}>0=P_{w}\left(b_{2}, b_{1}\right)$. Similarly, we have $P_{w}\left(b_{2}, b_{3}\right)=\frac{1}{8}>0=P_{w}\left(b_{3}, b_{2}\right)$. Consequently, $b_{1} P b_{2}$ and $b_{2} P b_{3}$. Nevertheless, $P_{w}\left(b_{3}, b_{1}\right)=\frac{3}{8}>\frac{1}{4}=P_{w}\left(b_{1}, b_{3}\right)$. So $b_{3} P b_{1}$. This result 
is related to the presence of an indifference threshold associated to an important weight (for criterion 2). In other words, while the difference on criterion 2 is low, it is not taken into account. As soon as this difference is greater than $q_{2}$ it is taken into account and its related weight is quite important.

Even if no indifference thresholds are considered, the problem remains due to the presence of $p_{j}$. Let us now consider that $w_{1}=\frac{2}{5}, w_{2}=\frac{3}{5}, q_{j}=$ $0, p_{j}=2$. In this case, we have: $P_{w}\left(b_{1}, b_{2}\right)=\frac{2}{5}>\frac{3}{10}=P_{w}\left(b_{2}, b_{1}\right)$ and $P_{w}\left(b_{2}, b_{3}\right)=\frac{2}{5}>\frac{3}{10}=P_{w}\left(b_{2}, b_{3}\right)$. Consequently, $b_{1} P b_{2}$ and $b_{2} P b_{3}$. Nevertheless, $b_{3} P b_{1}$ since $P_{w}\left(b_{3}, b_{1}\right)=\frac{3}{5}>\frac{2}{5}=P_{w}\left(b_{1}, b_{3}\right)$.

\section{- Partially closed nature of the system}

Finally a distinctive feature of the model that needs to be underlined is its partially closed nature. As mentioned before, the parameters of our model are partially fitted during the auction as a consequence of the bids comparison. When the auctioneer states that $b_{i} \mathcal{S}(k) b_{j}$ the model is updated in such a way that the previous constraint is satisfied. This learning process permits him to fit his preference structure dynamically. On the other hand, this fitting is dependent from the bids proposed by the sellers, who are (explicitly or implicitly) using the preference structure available at time $k$ to select the bid(s) they will propose at time $(k+1)$. It is in that sense that we speak of the partially closed nature of the process: the bids serve to fit the preference structure of the auctioneer while the sellers use this preference structure to propose their bids. In such context, one may ask the question to compare the butterfly models obtained trough two instances of the same auction. If both of them will be compatible with the preferences of the auctioneer, the auction's outcomes may be affected by their nature. More formally, let $\mathcal{S}$ denote the best butterfly that is compatible with the auctioneer's preferences, i.e. the butterfly with the biggest comparability window and the smallest set of admissible weights that can be obtained. Let $\tilde{S}$ be a butterfly obtained through one specific auction. The question raised here is to compare $W S_{\tilde{S}}$ and $W S_{S}$, i.e. the final bids obtained when the auction is based on $S$ or on $\tilde{S}$. This will be partially addressed in section 5.4. 


\subsection{Properties of the butterfly model}

Now that the butterfly model has been described and criticized, we will study, in this section, its related theoretical properties.

First of all, in order to remain consistent with the theoretical framework introduced in the previous chapter, let us verify that the butterfly model respects the mixed transitivity assumption.

Proposition 11 Let $\mathcal{S}$ be a butterfly model. We have aS $b, b \preceq c \Rightarrow a \mathcal{S} c$

\section{Proof:}

If $a \mathcal{S}(k) b$ and $b \preceq c \Rightarrow d_{j}(a, c)=c^{j}-a^{j}=\left(c^{j}-b^{j}\right)+\left(b^{j}-a^{j}\right) \geq-\phi_{j}(k), \forall j=$ $1, . . q$. As a consequence, the constraints related to the discordance levels are satisfied.

Now, let us prove that $P_{w}(a, c) \geq P_{w}(c, a), \forall w \in A W(k)$. By contradiction, let us assume that $\exists \tilde{w} \mid P_{\tilde{w}}(c, a)>P_{\tilde{w}}(a, c)$.

- $d_{j}(a, c)=c^{j}-a^{j}=\left(c^{j}-b^{j}\right)+\left(b^{j}-a^{j}\right)=d_{j}(b, c)+d_{j}(a, b) \geq$ $d_{j}(a, b)$. Consequently, $P_{\tilde{w}}(a, c)=\sum_{j=1}^{q} \tilde{w}_{j} \cdot P_{j}\left(d_{j}(a, c)\right) \geq \sum_{j=1}^{q} \tilde{w}_{j} \cdot$ $P_{j}\left(d_{j}(a, b)\right)=P_{\tilde{w}}(a, b)$.

- $d_{j}(c, a)=a^{j}-c^{j}=\left(a^{j}-b^{j}\right)+\left(b^{j}-c^{j}\right)=d_{j}(b, a)+d_{j}(c, b) \leq$ $d_{j}(b, a)$. Consequently, $P_{\tilde{w}}(c, a)=\sum_{j=1}^{q} \tilde{w}_{j} \cdot P_{j}\left(d_{j}(c, a)\right) \leq \sum_{j=1}^{q} \tilde{w}_{j} \cdot$ $P_{j}\left(d_{j}(b, a)\right)=P_{\tilde{w}}(b, a)$.

Since, by assumption, $a \mathcal{S} b \Rightarrow P_{w}(a, b) \geq P_{w}(b, a), \forall w \in A W(k)$ which contradicts the fact that $P_{\tilde{w}}(b, a) \geq P_{\tilde{w}}(c, a)>P_{\tilde{w}}(a, c) \geq P_{\tilde{w}}(a, b)$.

Additionally, let us note that a very similar proof leads to state that if $\mathcal{S}$ represents a butterfly model, we have $a \preceq b, b \mathcal{S} c \Rightarrow a \mathcal{S} c$.

As already explained, the way the butterfly has been built ensures some consistency of the comparisons; if an offer $b_{i}$ is at least as good as another bid $b_{j}$ at time $k, b_{i} \mathcal{S}(k) b_{j}$, it will remain preferred or indifferent during the rest of the auction. This statement is formalized in the next proposition: 
Proposition $12 b_{i} \mathcal{S}(k) b_{j} \Rightarrow b_{i} \mathcal{S}(\tilde{k}) b_{j}, \forall \tilde{k} \geq k$.

Proof: If $b_{i} \mathcal{S}(k) b_{j} \Rightarrow d_{l}\left(b_{i}, b_{j}\right) \geq-\phi_{l}(k) \geq-\phi_{l}(\tilde{k}), \forall \tilde{k} \geq k . \quad b_{i}$ is thus comparable to $b_{j}$. Furthermore, as stated before, $A W(\tilde{k}) \subseteq A W(k) \Rightarrow$ $T_{\tilde{k}}\left(b_{i}, b_{j}\right) \geq T_{k}\left(b_{i}, b_{j}\right) \geq 0$.

An interesting feature of the model introduced is that it is both compatible with the "natural" dominance relation and the weighted sum. These constitute two extreme cases within the family of butterfly models.

Proposition 13 The dominance relation is a particular case of the butterfly model.

Proof: Let $A W(0)=\left\{w_{l} \mid w_{l}>0, \sum_{l=1}^{q} w_{l}=1\right\}$. Let us assume that $\phi_{j}(k)=0, q_{j}=0, p_{j}=\max \left\{E_{j}\right\}, \forall j=1, . ., q, \forall k$. If $b \preceq \tilde{b} \Rightarrow d_{j}(b, \tilde{b}) \geq$ $0 \forall j=1, . ., q \Rightarrow P_{w}(b, \tilde{b}) \geq P_{w}(\tilde{b}, b) \forall w \in A W(k)$.

Let us now assume $T(b, \tilde{b}) \geq 0$ and $\exists j \mid b^{j}>\tilde{b}^{j}$. It is easy to see that $e_{j} \in$ $A W(0)$. Furthermore, $T(b, \tilde{b}) \leq 1_{j} \cdot\left(P_{j}\left(d_{j}(b, \tilde{b})\right)-P_{j}\left(d_{j}(\tilde{b}, b)\right)\right) \leq\left(\max \left(0, \tilde{b}^{j}-\right.\right.$ $\left.\left.b^{j}\right)-\max \left(0, b^{j}-\tilde{b}^{j}\right)\right) \leq-\left(b^{j}-\tilde{b}^{j}\right)<O$.

Proposition 14 The weighted sum is a particular case of the butterfly model.

Proof: Let us consider $\mathrm{k}$ such that $\forall l=1, \ldots, q \phi_{l}(k)=\infty, q_{j}=0, p_{j}=$ $\max \left\{E_{j}\right\}$ and $A W(k)=\left\{w_{l}(k)=\lambda_{l}\right\}$ where $\lambda_{l}$ are parameters fixed by the auctioneer such that $\lambda_{l}>0$ and $\sum_{l=1}^{q} \lambda_{l}=1$.

If $b \mathcal{S}(k) \tilde{b} \Leftrightarrow \sum_{j=1}^{q} \lambda_{j} \cdot\left(P_{j}\left(d_{j}(b, \tilde{b})-P_{j}\left(d_{j}(\tilde{b}, b)\right) \geq 0 \Leftrightarrow \sum_{j=1}^{q} \lambda_{j} \cdot\left(\max \left\{0, \frac{\tilde{b}^{j}-b^{j}}{p_{j}}\right\}-\right.\right.\right.$ $\left.\max \left\{0, \frac{b^{j}-\tilde{b}^{j}}{p_{j}}\right\}\right) \geq 0 \Leftrightarrow \sum_{j \mid \tilde{b}^{j} \geq b^{j}}\left(\frac{\tilde{b}^{j}-b^{j}}{p_{j}}\right) \cdot \lambda_{j}+\sum_{j \mid b^{j} \geq \tilde{b}^{j}}\left(\frac{\tilde{b}^{j}-b^{j}}{p_{j}}\right) \cdot \lambda_{j} \geq 0 \Leftrightarrow$ $\sum_{j=1}^{q}\left(\frac{\tilde{b}^{j}-b^{j}}{p_{j}}\right) \cdot \lambda_{j} \geq 0 \Leftrightarrow \sum_{j=1}^{q} \tilde{\lambda}_{j} \cdot \tilde{b}^{j} \geq \sum_{j=1}^{q} \tilde{\lambda}_{j} \cdot b^{j}$ where $\tilde{\lambda}_{j}=\frac{\lambda_{j} / p_{j}}{\sum_{k=1}^{q} \lambda_{k} / p_{k}}$. 
In section 5.2.4, we have already argued that relation $\mathcal{S}$ was not acyclic due to the use of preference and indifference thresholds. The following proposition illustrates that the asymmetric part of $\mathcal{S}$ is nevertheless acyclic under specific assumptions.

Proposition 15 If $q_{j}=0$ and $p_{j}=\max \left\{E_{j}\right\}$, relation $P$ is acyclic.

Proof: Let $b_{1} P b_{2} P b_{3} P \ldots P b_{n}$. Let us assume that $b_{n} P b_{1}$. Of course, we have $b_{1}^{j}-b_{n}^{j} \geq-\phi_{j}$, otherwise this assumption is meaningless. Under these assumptions, we have already shown that $b P \tilde{b} \Leftrightarrow \sum_{j=1}^{q} w_{j} \cdot \frac{b^{j}}{p_{j}}<\sum_{j=1}^{q} w_{j} \cdot \frac{\tilde{b}^{j}}{p_{j}}$ $\forall w \in A W$. Consequently, $\sum_{j=1}^{q} w_{j} \cdot \frac{b_{1}^{j}}{p_{j}}<\sum_{j=1}^{q} w_{j} \cdot \frac{b_{2}^{j}}{p_{j}}<\ldots<\sum_{j=1}^{q} w_{j} \cdot \frac{b_{n-1}^{j}}{p_{j}}<$ $\sum_{j=1}^{q} w_{j} \cdot \frac{b_{n}^{j}}{p_{j}} \forall w \in A W$ which is in contradiction with the aforementioned property.

Finally, let us show that the convexity of the set of admissible weights $A W$ induces the convexity of the set of the values $P_{w}(a, b)$. Let $\bar{P}(a, b)=$ $\max _{w} P_{w}(a, b)=\sum_{j=1}^{q} \bar{w}_{j} \cdot P_{j}\left(d_{j}(a, b)\right)$ and $\underline{P}(a, b)=\min _{w} P_{w}(a, b)=\sum_{j=1}^{q} \underline{w}_{j}$. $P_{j}\left(d_{j}(a, b)\right)$.

Proposition $16 \forall p \in[\underline{P}(a, b), \bar{P}(a, b)], \exists \tilde{w} \in A W(k)$ such that $p=P_{\tilde{w}(a, b)}$

Proof: Let $p=\alpha \cdot \underline{P}(a, b)+(1-\alpha) \cdot \bar{P}(a, b)=\alpha \cdot \sum_{j=1}^{q} \underline{w}_{j} \cdot P_{j}\left(d_{j}(a, b)\right)+$ $(1-\alpha) \cdot \sum_{j=1}^{q} \bar{w}_{j} \cdot P_{j}\left(d_{j}(a, b)\right)=\sum_{j=1}^{q}\left(\alpha \cdot \underline{w}_{j}+(1-\alpha) \cdot \bar{w}_{j}\right) \cdot P_{j}\left(d_{j}(a, b)\right)=$ $\sum_{j=1}^{q} \tilde{w}_{j} \cdot P_{j}\left(d_{j}(a, b)\right)$, where $\tilde{w}=\alpha \cdot \underline{w}+(1-\alpha) \cdot \bar{w}$ which is an admissible weight due to the convexity of $A W$.

\section{$5.4 \quad$ Related questions}

This section is devoted to general questions regarding the model studied in this chapter. Therefore, problems related to preference elicitation, decrement steps and indifference thresholds, multicriteria bidding support tools and winning bids'robustness will be addressed in the next subsections. 


\subsubsection{Bids submitted and preference elicitation}

In the previous sections, we have argued that the auctioneer was able to update his preference structure during the auction thanks to bids comparisons. A critical question, related to this distinctive feature, is to evaluate if the model empirically obtained is close or not to the real preference structure of the auctioneer. This question will be the center of interest of this subsection.

Let us assume that the preferences of the auctioneer are perfectly represented by a butterfly model $\mathcal{S}$, and let $\tilde{\mathcal{S}}(k)$ be the butterfly model at time $k$ that is built during the auction as a consequence of the bids comparison (in this context we will assume that the auctioneer doesn't impose direct constraints). The question raised in this section is to evaluate if $\tilde{\mathcal{S}}(k)$ is close to $\mathcal{S}$, depending on the dimension space and the number of bids, k, submitted up to now. Algorithm 3 gives an outline of the procedure that was used to conduct these experiments. Note that, for simplicity reasons, we assume $q_{j}=0$ and $p_{j}=1$ for each criterion.

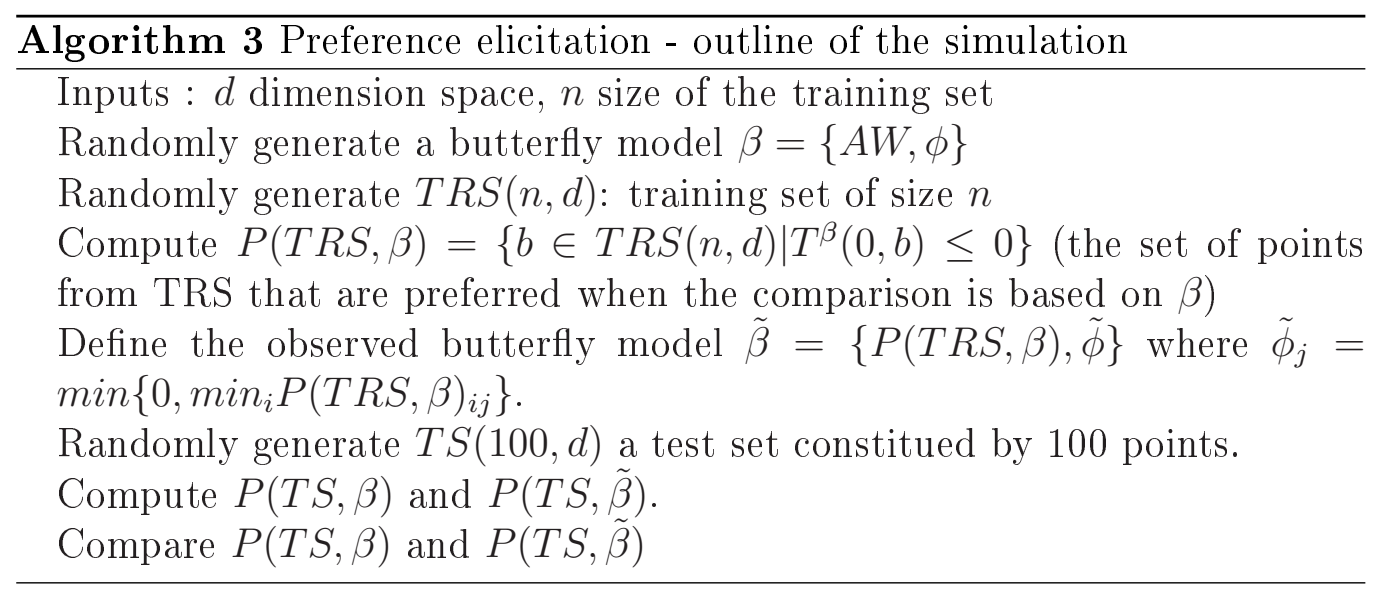

The test starts with a given value $d$ for the dimension space and a given size $n$ for the training set. All points generated during the simulation are drawn from a multivariate uniform distribution on $[-1 ; 1]^{n}$. The origin 0 is considered to be the reference point (for the comparisons); i.e. the butterfly model is centered on it. This assumption is not restrictive and can be easily extended to other points. Points that are dominating the origin or that dominate it, i.e. the positive and the negative orthant, are removed since their comparison with $\mathbf{0}$ is obvious. When generating training or test sets such points will never be considered. The first step of the algorithm is the random generation of a butterfly model. $\beta=\{A W, \phi\}$ represents the 
auctioneer's preferences, i.e. the most precise butterfly that can represent his preferences. To build this butterfly, we have assumed that $\phi_{j} \sim U_{[0,1]}$, i.e. $\phi_{j}$ is a random variable drawn from a uniform distribution on $[0,1]$. The set of admissible weights $A W$ is defined by $2^{d}-2$ vectors representing points that are preferred by $\mathbf{0}$. Each point must be in a different orthant. (One more time, we do not consider points that dominate or are dominated by 0. Eliminating these 2 orthants leads to $2^{d}-2$ different points) Once the butterfly model is generated, a training set of size $n$, noted $T R S(n, d)$, is built. $P(T R S, \beta)$ represents the set of points that are preferred by $\mathbf{0}$ when the comparison is based on $\beta$. During the bids comparison, the auctioneer has been reacting according to $\beta$ (we have assumed that his preferences were compatible with $\beta$ ). However, he does not know the explicit form of $\beta$. The observed butterfly $\tilde{\beta}$ is nothing else but the butterfly model that can be deduced from the comparisons done on the training set. In this case both the set of admissible weights $A \tilde{W}$ and the comparability window $\tilde{\phi}$ are deduced from the bids from $\operatorname{TRS}(n, d)$ that were preferred by $\mathbf{0}$. To compare $\beta$ and $\tilde{\beta}$, a test set constituted by 100 points is randomly generated. Finally, $P(T S, \beta)$ and $P(T S, \tilde{\beta})$ are generated and compared.

We have run simulations for values of $d$ ranging from 2 to 5 and for values of $n$ ranging from 2 to 30 . At first, the comparison was based on the similarity between both results: this means that we were looking for points of the test set that were classified in the same way (preferred by $\mathbf{0}$ or not) by $\beta$ and $\tilde{\beta}$. Figure 5.4 illustrates the mean percentage of correctly classified points (i.e. the number of correctly classified points divided by the total number of points $=100$ ) when the size of the training set evolves from 2 to 30. (For each combination of the parameters $n$ and $d, 100$ simulations have been run). As expected the bigger the training set, the higher this percentage. Nevertheless, it's interesting to remark that even if the size of the training set is small, the percentage remains relatively high (around 0.75 ). This seems counterintuitive at first sight, since $\tilde{\beta}$ is fitted on a relatively poor information. However, this effect is explained by the fact that we are considering similarities between the two models without considering the status of the classified point; points rejected in both models are also taken into account in our measure. However, due to the simulation assumptions $\left(\phi \sim U_{[0,1]}\right)$, on average 50 percent of the points generated will lie outside of the comparability window. On the other hand, by default, $\tilde{\beta}$ is initially defined using a comparability window restricted to the dominated points. If no further information regarding the preferred points is provided, any other points will be considered as incomparable. As already mentioned, on average, 50 percent of the points will lie in this area. 


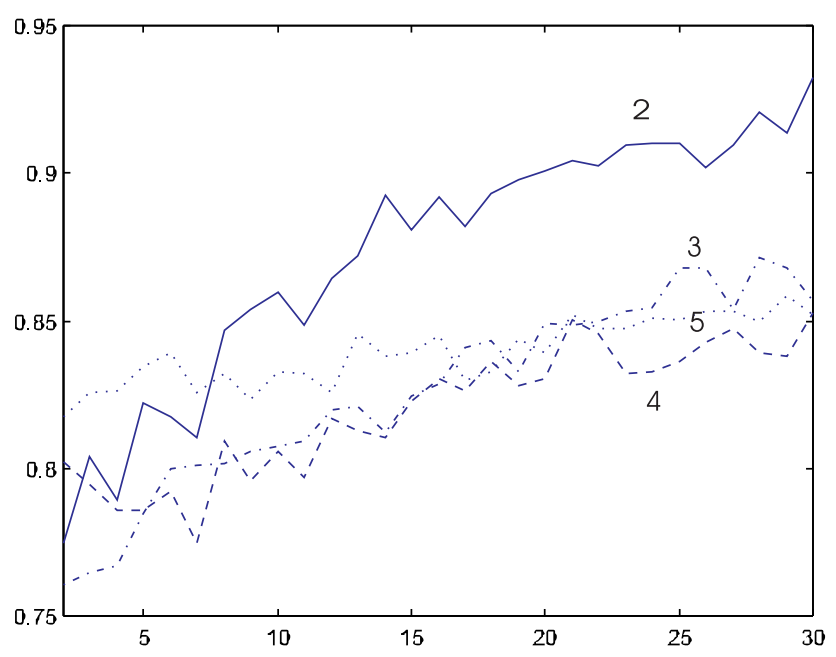

Figure 5.4: Evolution of the mean percentage of good "classifications" when the size of the training set evolves from 2 to 30 . The different curves correspond to values of $\mathrm{d}$ ranging from 2 to 5 .

In order to criticize the results shown on figure 5.4, we have computed the number of preferred points, when $\beta$ was used, that were effectively preferred when $\tilde{\beta}$ was used. As shown on figure 5.5, these results are inline with what was expected for 2 or 3 dimensions. However when the dimension increases to 4 or 5 , the evolution is less significant and it seems that the size of the training set needs to be greater to get a good picture of the preference structure. This effect is due to the fact the number of orthants to consider is exponential in the dimension.

Finally, it is worth noting that, as expected, in both simulations, the variance of the results is decreasing when the size of the training set increases.

\subsubsection{Bid decrement and indifference thresholds}

A question that has not yet been addressed in the present work is related to bid decrements (bid increment in forward auctions). As stressed in chapter 1 , it is nevertheless a fundamental point in the analysis of auctions.

Usually, the bid decrement is fixed with reference to a total cost or the level of a multi-attribute utility function. By doing so, the auctioneer may 


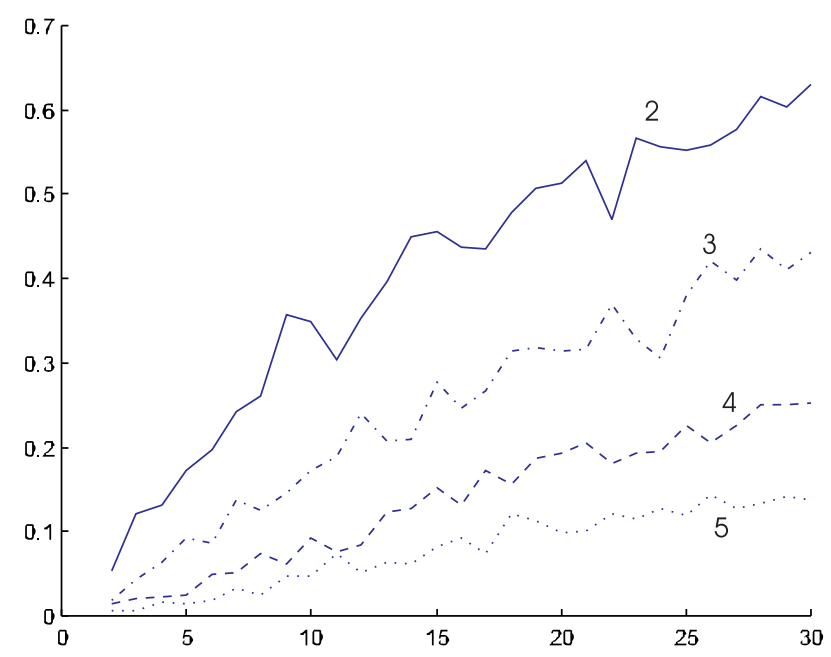

Figure 5.5: Evolution of the mean percentage of points that were classified as preferred by both models $(\beta$ and $\tilde{\beta}$ ) when the size of the training set evolves from 2 to 30 . The different curves correspond to values of $\mathrm{d}$ ranging from 2 to 5 .

state, for instance, that the difference between a new bid and any bid from the auction history, in terms of utility, must be greater or equal to a fixed utility step, noted $\epsilon$ (see figure 5.6). From a practical point of view, the interpretation of this parameter by the auctioneer is, nevertheless, not always intuitive (however, this critic is less significant when using a total cost approach).

In our context, this way of doing is not well-suited since the auctioneer's preferences may evolve during the auction. Imposing, for instance, that $b S(k) \tilde{b}$ iff $P_{w}(b, \tilde{b}) \geq P_{w}(\tilde{b}, b)+\epsilon$ is, in our view, not meaningful since the weights $w$ are not fixed prior to the auction. With these settings, the interpretation of the $\epsilon$ parameter would not be obvious. Moreover the use of indifference thresholds in the computation of the preference degrees per criterion, implicitly leads us to take into account a "minimum bid decrement" per criterion.

The relation between the notion of bid decrement and indifference thresholds will be the core of this section. This comparison is far from obvious since the intrinsic nature of these two notions is quite different. On the one hand, indifference thresholds are commonly used in the multicriteria decision aid community to establish levels below which the difference between two alter- 


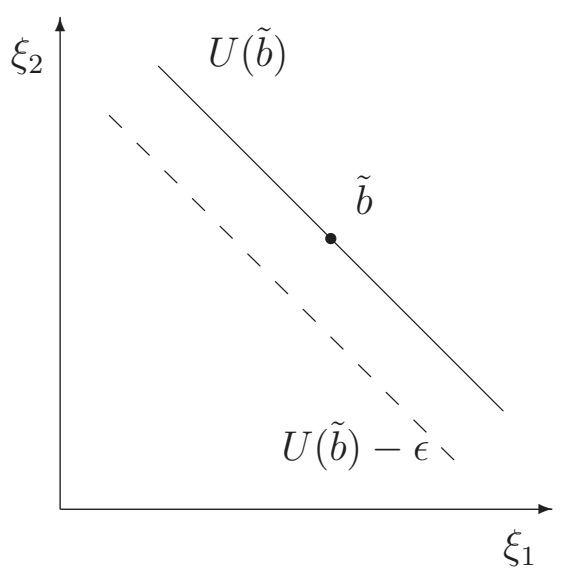

Figure 5.6: Illustration of a decrement step $\epsilon$ in a multi-attribute utility auction.

natives (on a specific criterion) is too small to be taken into account in the pairwise comparisons process. On the other hand, in an auction context, bid decrements (increment) are used to determine if a new bid is acceptable or not, i.e. if the difference between this offer and the previously submitted ones is greater than or equal to it.

In the context of multicriteria auctions, the acceptation rule requires that any new bid $b$ must not be preferred or indifferent to bids $\tilde{b}$ that have been previously accepted. Consequently, when using a butterfly model, indifference thresholds implicitly intervene in the acceptation rule. The next proposition clarifies this point.

Proposition 17 Let $B A$ be a butterfly auction. A necessary condition for $a$ bid $b$ to be an acceptable offer at time $k$ is that:

$$
\forall \tilde{b} \in H_{M A}(k) \exists j \in\{1, . ., q\} \quad \mid \tilde{b}_{j}-b_{j}>q_{j}
$$

Proof: Let us assume that $\exists \tilde{b} \in H_{M A}(k) \mid \forall j \in\{1, . ., q\}: \tilde{b}_{j}-b_{j} \leq q_{j} \rightarrow$ $d_{j}(b, \tilde{b})=0 \forall j \rightarrow P_{w}(b, \tilde{b})=0 \forall w$. Moreover, since $q_{j} \leq \phi_{j}(k)$, we have $b_{j}-\tilde{b}_{j} \geq-q_{j} \geq-\phi_{j}$. Consequently, $\tilde{b} S(k) b$ and $b$ is not an acceptable bid.

With respect to the previous result, the interpretation of indifference thresholds in terms of bid decrements is somewhat clearer. Moreover, due 


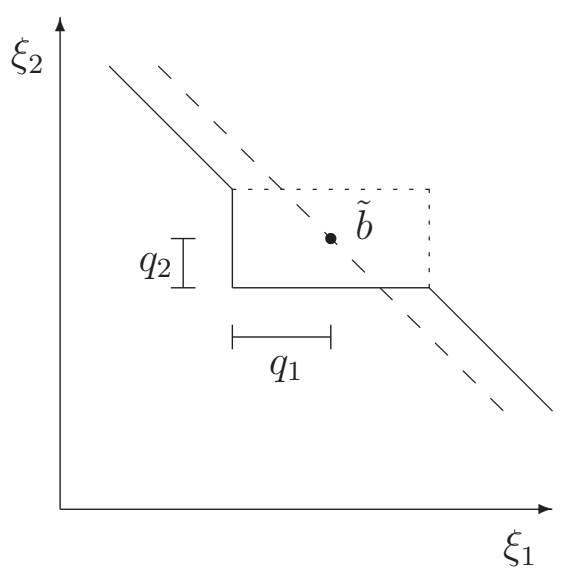

Figure 5.7: Illustration of indifference thresholds in a particular butterfly model.

to their uni-criterion nature, their practical use is less abstract than a global (multi-attribute utility) bid decrement.

To further illustrate the relation between indifference threshold and decrement step let us consider figure 5.7. The settings are the same as in figure 5.6; $w_{1}=w_{2}=\frac{1}{2}$. The continuous line delimits the set of points that are preferred or indifferent to $\tilde{b}$ while the dashed line reminds the multi-attribute iso-utility of figure 5.6.

\subsubsection{Multicriteria bidding support tool}

In the context of multi-attribute auctions, the cognitive complexity of the bidding process remains a fundamental problem to manage. Both the number and the nature of the parameters characterizing the aforementioned model and the rules defining multicriteria auctions move the field away from the simple beat the quote rule characterizing classic auctions. Without adapted bidding support tools, bidders are likely to avoid using these kinds of mechanisms. In this section, we present an algorithm to support the bidder in his bidding activity.

Let us consider a butterfly auction $B A$ at time $k$. Seller $s_{i}$ wants to submit a new bid $b_{i}$. We assume, here, that $s_{i}$ has already specified the levels of all attributes except the $j^{\text {th }}$ one. For convenience this will be referred to as $b_{i}^{-j}$. The question, asked in this context, is to determine the maximum 
level $b_{i}^{j} \in E_{j}$ such that $b_{i}$ satisfies the multicriteria auction rule (i.e. is not outranked by any bid in $\left.H_{B A}(k)\right)$.

First of all, let us remark that the level $b_{i}^{j}$ is only influenced by the bids $\tilde{b} \in H_{B A}(k)$ such that $d_{j}(\tilde{b}, b) \geq-\phi_{j}(k), \forall j \neq i$. On the contrary, if $\exists j$ such that $d_{j}(\tilde{b}, b)<-\phi_{j}(k)$, i.e. $b^{j}-\tilde{b}^{j}<-\phi_{j}(k)$ we will have $\tilde{b} \neg \mathcal{S} b$ whatever the values $b_{i}^{j}$. The subset of bids $\tilde{b} \in H_{B A}(k)$ that are likely to influence the level $b_{i}^{j}$ will be denoted by $\mathcal{C}\left(b_{i}^{-j}, H_{B A}(k)\right)$. More formally,

Definition $21 \mathcal{C}\left(b_{i}^{-j}, H_{B A}(k)\right)=\left\{\tilde{b} \in H_{B A}(k) \mid d_{j}(\tilde{b}, b) \geq-\phi_{j}(k), \forall j \neq i\right\}$.

Of course, if $\mathcal{C}\left(b_{i}^{-j}, H_{B A}(k)\right)=\emptyset$ the solution of the problem is obvious and $b_{i}^{j}=\max \left(E_{j}\right)$. Let us further assume that:

- if $b_{i}^{j}=\min \left(E_{j}\right), \nexists \tilde{b} \in \mathcal{C}\left(b_{i}^{-j}, H_{B A}(k)\right) \mid \tilde{b} \mathcal{S}(k) b$. If this is not the case, no value of $b_{i}^{j}$ will lead to an acceptable offer.

- if $b_{i}^{j}=\max \left(E_{j}\right), \exists \tilde{b} \in \mathcal{C}\left(b_{i}^{-j}, H_{B A}(k)\right) \mid \tilde{b} \mathcal{S}(k) b$. If this is not the case, the solution of the problem is obvious and we choose $b_{i}^{j}=\max \left(E_{j}\right)$.

Under these assumptions, we will apply a dichotomic search in order to determine the level $b_{i}^{j}$ to propose to $s_{i}$. This approach is sketched in algorithm 4. This choice is motivated by the following property:

Proposition 18 If $\exists b^{j} \in\left[\min \left(E_{j}\right), \max \left(E_{j}\right)\right]$ such that when $b_{i}^{j}=b^{j}$, $\nexists \tilde{b} \in$ $\mathcal{C}\left(b_{i}^{-j}, H_{B A}(k)\right) \mid \tilde{b} \mathcal{S}(k) b_{i}$ then $\forall b_{i}^{j} \leq b^{j}, \nexists \tilde{b} \in \mathcal{C}\left(b_{i}^{-j}, H_{B A}(k)\right) \mid \tilde{b} \mathcal{S}(k) b_{i}$.

\section{Proof:}

Let us assume that $\exists b_{i}^{j}$ such that $\nexists \tilde{b} \in \mathcal{C}\left(b_{i}^{-j}, H_{B A}(k)\right) \mid \tilde{b} \mathcal{S}(k) b_{i}$. Let us consider $\tilde{b}_{i}^{j} \leq b_{i}^{j}$ and $\tilde{b}_{i}$ denotes the bid proposed by $s_{i}$, i.e. $b_{i}^{-j}$ where the $j^{t h}$ component is $\tilde{b}_{i}^{j}$. Of course, $\tilde{b}_{i} \preceq b_{i}$. By contradiction, let us assume that $\exists \tilde{b} \in \mathcal{C}\left(b_{i}^{-j}, H_{B A}(k)\right)$ such that $\tilde{b} \mathcal{S}(k) \tilde{b}_{i}$. As a corollary of the mixed transitivity assumption, we have $\tilde{b} \mathcal{S}(k) \tilde{b}_{i}, \tilde{b}_{i} \preceq b_{i} \Rightarrow \tilde{b} \mathcal{S}(k) b_{i}$ which is in contradiction with the aforementioned assumption. 


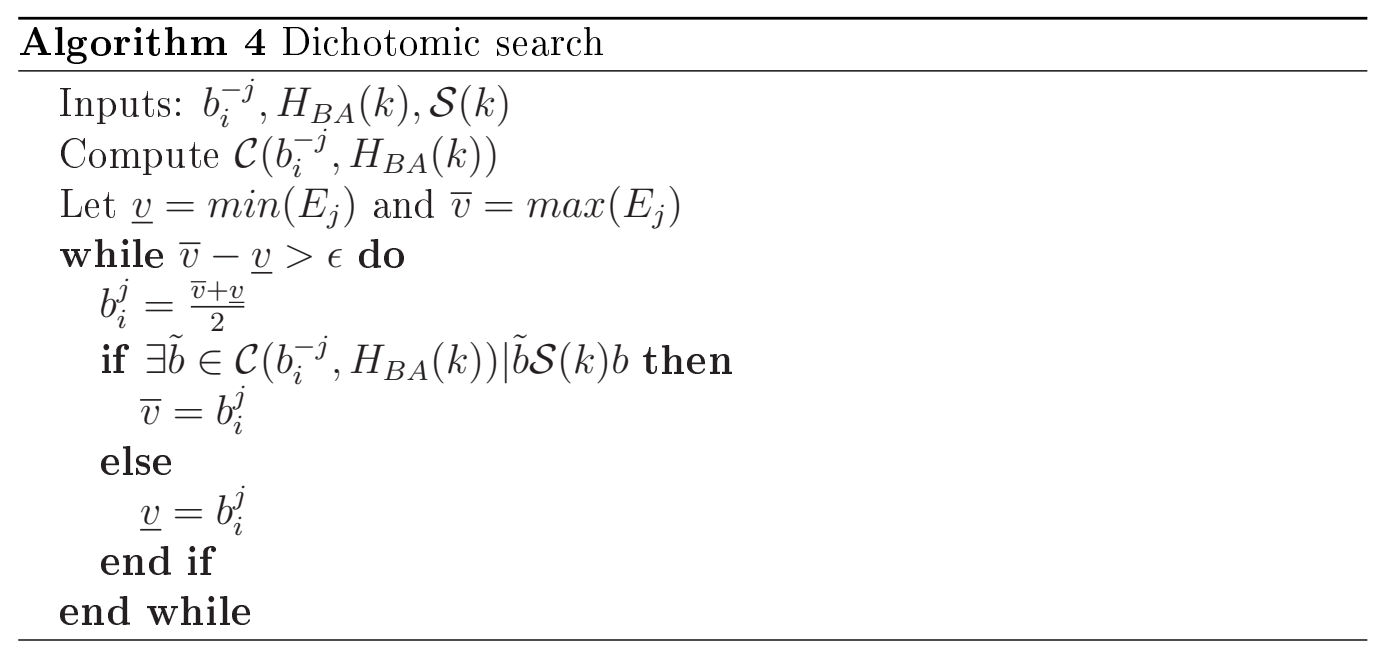

In other words, the interval $\left[\min \left(E_{j}\right), \max \left(E_{j}\right)\right]$ may be split into two distinct parts: values $b_{i}^{j}$ such that $\nexists \tilde{b} \in \mathcal{C}\left(b_{i}^{-j}, H_{B A}(k)\right) \mid \tilde{b} \mathcal{S}(k) b$ and values $b_{i}^{j}$ and values $b_{i}^{j}$ such that $\exists \tilde{b} \in \mathcal{C}\left(b_{i}^{-j}, H_{B A}(k)\right) \mid \tilde{b} \mathcal{S}(k) b$.

The parameter $\epsilon$, used in the convergence condition, can be interpreted as the maximum bid increment between the value $\widehat{b}_{i}^{-j}$ obtained by the algorithm and the optimal value $b_{i}^{-j}$. This value is set by the bidder.

Let us note that the algorithm will propose a value for $b_{i}^{-j}$ without taking into account the nature of $E_{j}$ (since the values of $E_{j}$ are treated as a numerical scale). Moreover, when $E_{j}$ is a discrete set the value returned by the algorithm is not necessarily an acceptable value. In such a situation, the biggest acceptable value that is strictly lower than the one returned by the algorithm has to be considered.

To conclude, let us stress that the previous developments are not peculiar to the butterfly model and remain valid within the general theoretical framework studied in the previous chapter. Finally, an illustration of this algorithm will be outlined in section 5.5.

\subsubsection{Some reflections about robustness}

As already stressed, one of the main distinctive features of our approach relies on the fact that the auctioneer may refine his preference structure during the auction. With respect to the comments presented in section 5.2.4, he may state, for instance that $b_{i} \mathcal{S} b_{j}$ while $b_{i}, b_{j} \in W S_{M A}(k)$ (and consequently $b_{i}, b_{j}$ were considered to be incomparable). This assertion will then be taken 
into account in the butterfly model; both the set of admissible weights and the discordance levels will be updated and the winning set will be reduced. Therefore, such events (caused by the auctioneer) do have impacts on the auction progress and on the auction results. Evaluating the consequences of these actions and identifying the (related) sensitive components of the auction is at the core of the section.

Let us remind the reader that, at time $k$, the auctioneer can only state propositions in the form $b_{i} \mathcal{S} b_{j}$ where $b_{i}, b_{j} \in W S_{M A}(k)$. If $\left|W S_{M A}(k)\right|=n$, there are $\frac{n \cdot(n-1)}{2}$ different statements. From the bidder perspective $b_{i} \mathcal{S} b_{j}$ will be referred to as a potential scenario noted $s c_{l}$ (i.e. the $l^{\text {th }}$ scenario).

First of all, let us, one more time, stress that a direct consequence of the way the outranking relation $\mathcal{S}$ has been built is that, once a bid is outranked at time $k$, it remains outranked whatever the forthcoming scenario $s c_{l}$. Consequently, the nature of "beaten" bids is not affected by the different potential scenarios.

From now on, our attention will be focused on the evolution of the winning set subject to the various scenarios. Let us assume that at time $k$, the winning set contains $n$ distinct offers $W S_{M A}(k)=\left\{b_{1}, b_{2}, \ldots, b_{n}\right\}$. Let $A$ be a $n \times \frac{n \cdot(n-1)}{2}$ binary matrix. $A_{i j}=1$ denotes that $b_{i}$ remains in the winning set if scenario $s c_{j}$ occurs (on the contrary if $A_{i j}=0, b_{i}$ is no longer in the winning set if scenario $s c_{j}$ occurs).

It is obvious that the analysis of the matrix $A$ permits to extract crucial information regarding the evolution of the offers belonging to $W S_{M A}(k)$. The following definition helps to further characterize these bids.

Definition $22 b_{i} \in W S_{M A}(k)$ is a non implicitly preferred bid iff $\sum_{j=1}^{C_{2}^{n}} A_{i j}=$ $C_{2}^{n}-(n-1)$

Intuitively, a non implicitly preferred bid is a bid that cannot be eliminated from the winning set unless the auctioneer explicitly states it. On the contrary, implicitly preferred bids are bids that could be implicitly eliminated from the winning set as a consequence of a statement defined on other bids.

Let $n_{i}=\frac{n \cdot(n-1)}{2}-\sum_{j=1}^{C_{2}^{n}} A_{i j}$ be the number of scenarios where $b_{i}$ is not in the winning set anymore. Due to the fact that we explicitly consider all the possible pairwise comparisons between offers from the winning set, we have: $n_{i} \geq(n-1), \forall i=1, \ldots, n$. Moreover, $n_{i}=(n-1)$ only for bids $b_{i}$ that are non implicitly preferred. 
As already argued, our aim, here, will be to detect bids that are too sensitive with respect to the various scenarios. One way to detect such bids is to verify if the following constraint is satisfied or not:

$$
n_{i} \geq K
$$

Of course, there are many ways to define the bound $K$. One could, for instance, imagine that $K=\alpha \cdot C_{2}^{n}$, i.e. a certain percentage, noted $\alpha$, of all the potential scenarios. On the other hand, $K$ could be fixed whatever the number of potential scenarios. In the following developments, we will consider an approach based on confidence intervals.

If we assume that the probability of being eliminated from the winning set in a specific scenario $s c_{l}$ is a Bernoulli distribution of parameter $\hat{p}_{l}=\frac{\sum_{i=1}^{n} A_{i l}}{n}$ and if we apply the central limit theorem, we get the following rule: $b_{i}$ is judged to be too sensitive with respect to the potential scenarios if

$$
\sum_{j=1}^{C_{2}^{n}} A_{i j}>\sum_{j=1}^{C_{2}^{n}} \hat{p}_{j}+\mu_{\epsilon} \sqrt{\sum_{j=1}^{C_{2}^{n}} \hat{p}_{j}\left(1-\hat{p}_{j}\right)}
$$

where $\mu_{\epsilon}$ represents the $95 \%$ or $90 \%$ percentile of a Normal distribution.

Moreover, let us note that the values $\hat{p}_{j}$ permit to characterize the different scenarios. The higher the value $\hat{p}_{j}$ the higher the number of implicitly preferred bids in this scenario.

Of course, all the previous developments rely on the assumption that the matrix $A$ is available. The computation of this matrix is nevertheless time consuming (especially when the number of bids in the winning set is large) and this delay has to be related to the time separating two bids.

\subsection{An illustrative example}

The last section is devoted to an example. Our aim is, first of all, to illustrate the model discussed in the present chapter and its distinctive features. Consequently, a number of simplifications have been made in the following developments. 
Table 5.1: Seller's best contract evaluated according to the 2 criteria

\begin{tabular}{|l|cc|}
\hline & $p_{1}\left(\xi_{1}\right)$ & $p_{2}\left(\xi_{2}\right)$ \\
\hline$\xi\left(v_{1}\right)$ & 0.125 & 0.25 \\
$\xi\left(v_{2}\right)$ & 0.135 & 0.15 \\
$\xi\left(v_{3}\right)$ & 0.13 & 0.2 \\
\hline
\end{tabular}

Table 5.2: Mobile phone contract procurement auction. Application of the butterfly model. Illustration of one potential auction realization.

\begin{tabular}{|c|cccc|}
\hline Step & Bid id. & $\xi_{1}$ & $\xi_{2}$ & WS \\
\hline 1 & $b_{1}(1)$ & 0.18 & 0.3 & $\left\{b_{1}(1)\right\}$ \\
2 & $b_{2}(1)$ & 0.18 & 0.28 & $\left\{b_{2}(1)\right\}$ \\
3 & $b_{3}(1)$ & 0.18 & 0.26 & $\left\{b_{3}(1)\right\}$ \\
4 & $b_{1}(2)$ & 0.16 & 0.26 & $\left\{b_{1}(2)\right\}$ \\
5 & $b_{2}(2)$ & 0.18 & 0.24 & $\left\{b_{1}(2), b_{2}(2)\right\}$ \\
6 & $b_{2}(3)$ & 0.14 & 0.26 & $\left\{b_{2}(3)\right\}$ \\
7 & $b_{3}(2)$ & 0.13 & 0.24 & $\left\{b_{3}(2)\right\}$ \\
8 & $b_{2}(4)$ & 0.16875 & 0.2 & $\left\{b_{2}(4), b_{3}(2)\right\}$ \\
9 & $b_{2}(5)$ & 0.14 & 0.2 & $\left\{b_{2}(\overline{5})\right\}$ \\
\hline
\end{tabular}

Let us consider, once again, the example of the mobile phone contract procurement introduced in the previous chapter. In what follows, we will even simplify the problem by considering only two criteria: the price $p_{1}$ per minute paid while the total communication time does not exceed 100 hours a month and $p_{2}$ the price per minute paid if this time limit is exceeded (with respect to the aforementioned example, the extra communication time offered by the operators is not taken into account anymore). The valuations of the three sellers participating to the auction are reminded in table 5.1.

The auctioneer states that the intra-criterion information is the same for both criteria: $q_{i}=0.01$ and $p_{i}=0.05, i \in\{1,2\}$. In other words, $B$ expresses the fact that a difference lower than or equal to 0.6 euros per hour $(0.01$ per minute) is negligible while a difference greater than or equal to 3 euros per hour leads to a strict preference on the related criterion. To remain consistent with the previous subsections, $\phi_{j}=0.01, j \in\{1,2\}$. Table 5.2 illustrates a potential auction realization. 
When the auction starts, the auctioneer hasn't specified values for the weights; $w_{1} \in[0,1]\left(w_{2}=1-w_{1}\right)$ and, as already mentioned, $\phi_{j}=0.01, j \in$ $\{1,2\}$. Seller $s_{1}$ is the first one to propose a bid. Seller $s_{2}$ reacts and decide to propose a new bid by improving the value of $p_{2}$. Since $q_{2}=0.01$, this value must be strictly lower than 0.29. Otherwise, the bid would be outranked by $b_{1}(1)$. Consequently, he proposes $b_{2}(1)=(0.18,0.28)$. (This illustrates the parallel between indifference thresholds and decrement steps). Until step 4 , no extra-information is needed since the offers are improved according to a strict dominance relation. Then, at time 5 , seller $s_{2}$ propose a bid $b_{2}(2)$ that is better than the current winning bid $b_{1}(2)$ regarding the value of $p_{2}$ but worse for $p_{1}$. Both bids are present $W S(5)$ and $B$ decides to choose between them; he states $b_{1}(2) \mathcal{S} b_{2}(2)$ and the model is refined. It is obvious to verify that the parameter of the model are now: $\phi_{1}=0.01, \phi_{2}=0.02$ and $w_{1} \in[0.5,1]$. Even if he has just been excluded from the winning set, seller $s_{2}$ offers a new bid $b_{2}(3)$ at time 6 and finally beat $b_{1}(2)$. At time 7 , seller $s_{3}$ reacts and offer $b_{3}(2)=[0.13,0.24]$. Compared with $b_{2}(3)$, this last offer is strictly better for both criteria and exceeds the indifference threshold for at least one criterion: consequently it remains the unique bid in $W S(7)$. Now, $s_{1}$ cannot propose any new bids since $b_{3}(2) \mathcal{S} \xi\left(v_{1}\right)$ and so, quits the auction. At time $8, s_{2}$ does not know which bid to propose. He decides to improve the value of $p_{2}$ to 0.2 and uses the decision support tool described in section 5.4 .3 to determine the value of $p_{1}$ (this step will be further discussed here below). Finally, he proposes $b_{2}(4)=[0.16875,0.2]$ and enters the winning set. $B$ decides between these two bids and states $b_{3}(2) \mathcal{S} b_{2}(4)$. As a consequence the parameters of the model are updated and we have: $\phi_{1}=0.01, \phi_{2}=0.02$ and $w_{1} \in\left[\frac{24}{47}, 1\right]$. At step 9 , seller $s_{2}$ proposes $b_{2}(5)=[0.14,0.2]$ and, since $s_{3}$ cannot propose any new bid, wins the auction.

Let us, now, consider the detail of step 8. As already said, seller $s_{2}$ decides to use the bidding support tool to offer a new bid. He decides to fix the value of $p_{2}$ to 0.20 and wonders what would be the higher value of $p_{1}$ that still allows the resulting bid to satisfy the auction rule (we assume that the precision degree $\epsilon$ is set to 0.01). First of all, it is easy to verify that, in this case, $\mathcal{C}\left(b_{i}^{-j}, H_{B A}(k)\right)=\left\{b_{2}(2), b_{3}(2)\right\}$ since for all the others bids in $H_{B A}(k)$ discordance happens regarding $p_{2}$.

Table 5.3 illustrates the progress of algorithm 4. For simplicity, we assume, here, that $E_{1}=[0.1,0.2]$. If $p_{1}=0.1$ the resulting bid satisfies the auction's rules while it is not the case when $p_{1}=0.2$. Consequently, the value $\frac{0.1+0.2}{2}=0.15$ is tested. If $p_{1}=0.15$ the resulting bid is accepted. So, the research interval is reduced to $[0.15,0.2]$. Subsequent steps use the same 
Table 5.3: Mobile phone contract procurement auction. Illustration of algorithm 4.

\begin{tabular}{|c|cccc|}
\hline Iteration & Lower bound & Upper bound & Tested point & Test result \\
\hline 1 & 0.10 & 0.20 & 0.15 & 1 \\
2 & 0.15 & 0.20 & 0.175 & 0 \\
3 & 0.15 & 0.175 & 0.1625 & 1 \\
4 & 0.1625 & 0.175 & 0.16875 & 1 \\
5 & 0.16875 & 0.175 & - & - \\
\hline
\end{tabular}

logic. Finally, the algorithm stops whenever the interval length is lower than or equal to $\epsilon$ and the lower bound is proposed to the seller: 0.16875 .

\subsection{Conclusion}

This chapter has been dedicated to the presentation and analysis of a particular auction model. Besides the management of potential incomparability between bids, the butterfly model allows the auctioneer to, progressively, refine his preference structure during the auction rather than to impose him to fix it before the auction. A number of specific issues related to this model have been discussed, its theoretical properties have been outlined and specific related questions have been treated. Finally, an example has permitted us to summarize and illustrate the previous points.

As already stressed (among others in section 5.2.4), a number of open questions remain and constitute as many directions for future researches. Nevertheless, a question that has not yet received much attention is the problem related to the time component of the problem. Indeed, in multicriteria auctions, the time separating two bids can be relatively low, leading to situations where the offers are received faster than the time needed to:

- select the winning offers

- (and / or) update the model

- (and / or) use the bidding support tool

- (and / or) study the robustness of the winning bids. 
Therefore, one may consider to proceed by using bidding rounds (during which the bids are (only) collected and between which the aforementioned computations are performed). Both the way these rounds are defined (i.e. their respective duration and the time separating two successive rounds) and their impact on the multicriteria auction results remain a subject to explore.

To conclude, let us stress that the present model will lead us to manage new auction contexts such as those constituted by different bidding niches. This notions will be formalized and empirically tested in the next chapter. 


\section{Chapter 6}

\section{Bidding Niches}

\subsection{Introduction}

In this chapter, our attention will be focused on the structure of the bidding space itself. More precisely, we will be interested in contexts where the bidding space is far from being homogeneous and, on the contrary, is shared within several bidding niches. In other words, submitted offers are concentrated in sub-regions of the bidding space while no bid exist for some combinations of the attributes ${ }^{1}$. To illustrate such situations, let us consider the three next examples:

\section{- The transport problem.}

Let us consider the case of a company that needs to import several containers of raw materials from a foreign country and decides to subcontract their transport. To select the subcontractor she organizes a multicriteria auction. Two criteria are considered to be relevant in this context: the total delay (expressed in days) and the price associated to the transport. Let us note that the total delay not only represents the duration of the transport but also the number of days to wait before the transport occurs. Figure 6.1 illustrates a potential realization of the auction. Clearly, the bidding space is not homogeneous and two areas can be identified: one characterized by a low price and an important delay and another characterized by a high price and a short delay. (see figure 6.1). This structure is a direct consequence of the fact that the

\footnotetext{
${ }^{1}$ as a consequence of production constraint for instance
} 


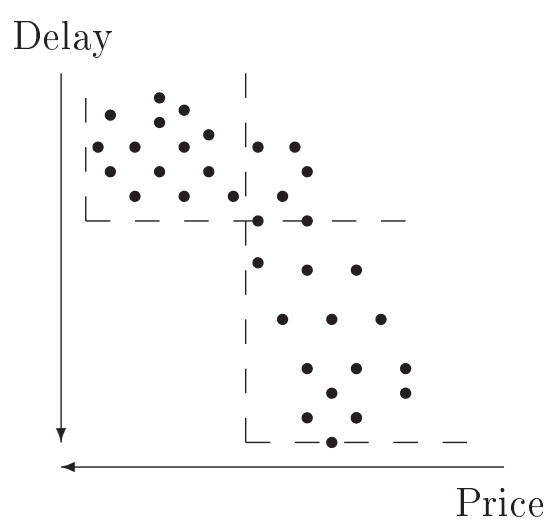

Figure 6.1: The transport problem: illustration of 2 bidding niches (delimited by the dashed lines).

transport can be conducted by trucks (short delays but expensive) or by barge (less expensive but more important delays).

\section{- The problem of the advertisement compain}

Let us consider the case of a company that decides to start an advertisement compain. In order to select the most appropriate advertisement option, she organizes a RFQ. For the sake of simplicity, let us assume that the offers are only evaluated on two criteria: the cost related to the advertisement (to be minimized) and an impact factor (to be maximized). The latter may be, for instance, the number of persons exposed to the advertisement that still remember the name of the company after a specific delay. In this context, distinct bidders are competing; television or radio operators, magazine managers, events organizers,... and provide different offers. Once all the bids have been collected, the company faces a situation illustrated on figure 6.2. Clearly, three distinctive sub-regions of the bidding space are outlined; low cost, low impact offers, medium cost, medium impact offers and high cost, high impact offers (these bidding niches correspond, roughly, to advertisements in magazine or during events, on radio and on television - nevertheless, let us note that advertisement in a major magazine may be competitive if compared to advertisement on television outside peak hours).

\section{- The cleaning contract problem}

A company desires to subcontract the cleaning of its offices. Therefore, 


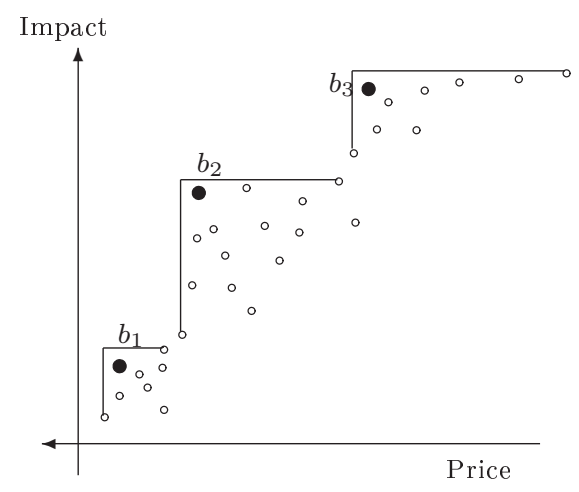

Figure 6.2: The advertisement problem: illustration of 3 bidding niches.

Term of the contract

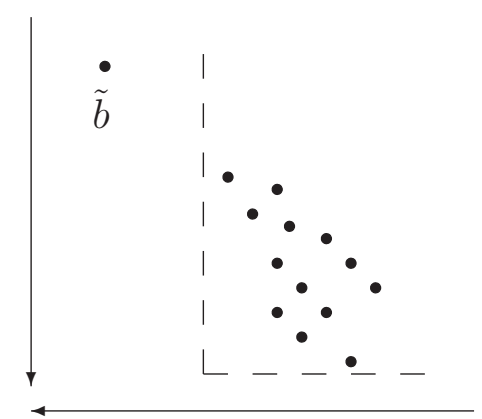

Fixed price per month

Figure 6.3: The procurement of a company's cleaning contract: illustration of outlying bids.

a procurement auction is organized. The buyer (i.e. the manager of the company who is in charge of the procurement) considers that two criteria have to be taken into account; the fixed price per month to pay for an everyday cleaning and the term of the contract. Figure 6.3 illustrates a potential realization of the auction. Clearly, the price decreases when the term of the contract increases and bid $\tilde{b}$ is an outlying bid. In other words, all the bids belong to the same bidding niche (denoted by the dashed line) except $\tilde{b}$. This situation results from the fact that nearly all the sellers are local companies; $\tilde{s}$ is the only foreign enterprise. Having cheaper labor costs, $\tilde{s}$ is able to propose lower prices. However, this company will not start a business in a new country unless a contract of minimum 5 years is guaranteed. 
In procurement contexts, the elicitation of bidding niches may be of great informational interest; particular structures may help to highlights production constraints or outlying bids. Furthermore, when the niches are clearly distinct, the procurer may prefer to select one competitive (and typical) offer for every niche rather to get a unique offer. For instance, in the advertisement problem, the bid taker is better of selecting both offers $b_{1}$ and $b_{2}$ than $b_{3}$. Indeed, the cost related to the simultaneous selection of $b_{1}$ and $b_{2}$ is lower than the cost of $b_{3}$ while this combination achieves a higher impact factor ${ }^{2}$.

Finally, in the context of Butterfly auctions, the detection of bidding niches may help the buyer to refine the model used. As shown in the previous chapter, one way to perform this task is to explicitly state $b \mathcal{S} \tilde{b}$ among two bids $b$ and $\tilde{b}$ that belong to the winning set. Of course, the comparison between every pair of bids in the winning set is often tedius and time consuming. To guide the buyer in this process, information about the bidding niches can be proposed. As a consequence, he will be able to limit the comparisons between bids belonging to the same niches.

Detecting groups structure within data sets is at the core of traditional clustering techniques. Therefore, a number of methods have already been proposed and analyzed in the literature. However, the problem considered in this paper is characterized by two distinctive features that have encouraged us to develop a new method rather than apply an existing one.

First of all, the offers are evaluated according to criteria (that have to be optimised). Traditional clustering techniques do not take into account this optimization constraint. Therefore, some methods have been developed to tackle this problem. They are referenced to as multicriteria clustering techniques $(38 ; 46 ; 82)$.

Secondly, the context considered in this contribution is related to procurement situations. As a consequence, the notion of competition between offers and groups of offers is crucial. Therefore, our aim will rather be "to detect groups of bids characterized by a high competition degree and that are not too competitive between themselves" than the traditional "detecting homogeneous groups of data that are heterogeneous between themselves".

In order to illustrate these assertions, let us remind the transport problem. Applying a traditional clustering technique to the offers illustrated on figure 6.4 will certainly lead to a unique cluster, even if 2 bidding niches co-exist (let

\footnotetext{
${ }^{2}$ let us note that this example illustrates an application to combinatorial multicriteria auctions.
} 


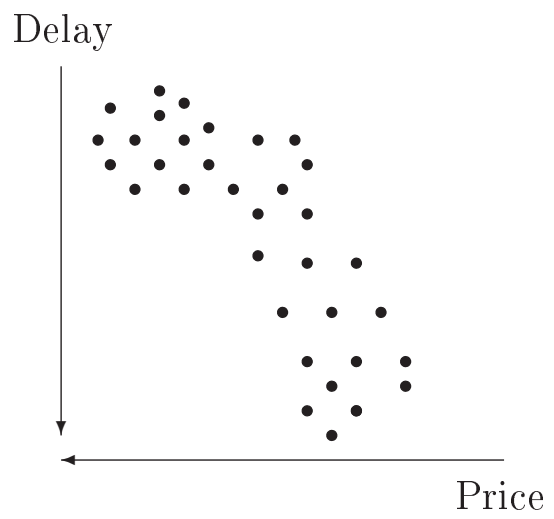

Figure 6.4: The transport problem (bis).

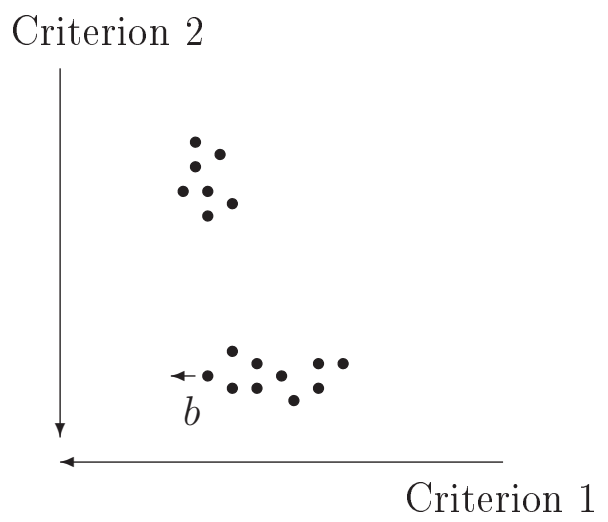

Figure 6.5: Illustration of a unique bidding niche.

us remind figure 6.1). In the same spirit, applying a traditional clustering algorithm to the bids illustrated on figure 6.5 will certainly lead to detect two clusters even if only one bidding niche is present. Indeed, if offer $b$ is slightly improved it will beat all the offers. Therefore, from the competitiveness point of view, some homogeneity remains and one cannot distinguish two distinct sets.

This chapter is organized as follows: section 6.2 is devoted to the formalization of the problem. The notions of competition between offers, cone partitions and IS partitions will be introduced and will allow us to further define bidding niches partitions. Then, in section 6.3, a hierarchical algorithm will be proposed for the detection of bidding niches partitions. The 
results of several empirical tests will be presented in section 6.4. Finally, we will end this chapter with some directions for future research in section 6.5.

To conclude this introduction, let us stress that this chapter is an extended version of the paper "Clustering the bidding space into niches: a hierarchical multicriteria clustering approach" (40).

\subsection{Formalization}

Until now, we have repeatedly (and maybe excessively) used the notion of partition of the bidding space into bidding niches without any formal definition. If the term bidding niche is explicit enough to give the reader an intuitive idea of what it depicts, we will, at last, formalize it in the next subsections.

As already stressed, the concept of competition between offers is at the core of the present chapter. This will be formalized in the next sections and then will allow us to define the notion of IS partitions and bidding niches partitions.

In what follows, $\mathcal{B}$ will denote a set of non dominated offers and $i(\mathcal{O})(\mathcal{O} \subseteq$ $\mathcal{B})$ will represent the ideal point associated to the set $\mathcal{O}$. Intuitively, $i(\mathcal{O})$ is the (virtual) bid that dominates (and thus beats) all the offers belonging to $\mathcal{O}$.

\subsubsection{Improvements'quantification}

Let us consider an offer $b \in \mathcal{B}$ and one improvement of $b$ noted $\tilde{b}=b-d$ where $d=\left(d^{1}, \ldots, d^{q}\right)$ (this non-negative vector indicates the improvement of $\tilde{b}$ over $b$ for each attribute). Of course, $\tilde{b} \prec b$. In what follows, we will quantify to what extend an offer $\tilde{b}$ dominates $b$. In order to obtain a global measure of these local improvements, we need to aggregate the components of $d^{3}$ :

$$
\iota: E^{1} \times \ldots \times E^{q} \rightarrow \mathbb{R}
$$

\footnotetext{
${ }^{3}$ let us note that, in this context, we restrict ourselves to improvements. In no way, we will compensate the degradation on one criterion by the improvement on another one.
} 


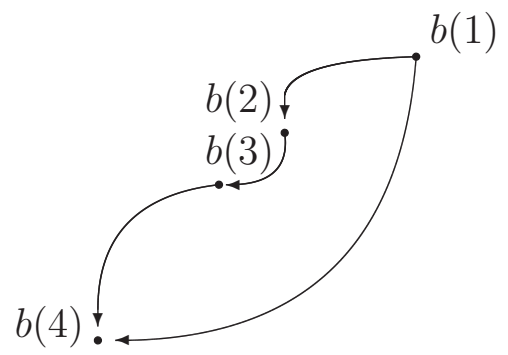

Figure 6.6: Additivity of the $\iota$ function

A reasonable property of $\iota$ is the addititivity. Indeed, if $d=\sum_{p=1}^{s} d_{p}$, the expression $\iota(d)=\iota\left(\sum_{p=1}^{s} d_{p}\right)=\sum_{p=1}^{d} \iota\left(d_{p}\right)$ where $d=\sum_{p=1}^{s} d_{p}\left(d_{p}^{j} \geq 0, \forall j, p\right.$, $d$ may be viewed as a succession of smaller improvements $d_{p}$ ). The additivity property expresses the fact that quantifying the global improvement can be decomposed into the sum of the quantification of smaller improvements (see figure 6.6).

A further issue that has to be taken into account when defining the $\iota$ function is related to the different natures of the scales $E^{j}$ (different units and / or different ranges). Therefore, we will consider a normalization factor $\delta=\left(\delta^{1}, \ldots, \delta^{p}\right)$ such that $\iota\left(\delta \cdot e_{j}\right)=1, \forall j=1, \ldots, p^{4}$. Intuitively, $\delta^{j}$ represents a standardized incremental step for the $j^{\text {th }}$ attribute. In other words, measuring an improvement of a quantity $\delta^{j}$ on the $j^{\text {th }}$ attribute is equivalent to measuring an improvement of a quantity $\delta^{k}$ on the $k^{t h}$ attribute.

A direct consequence of the previous statements is $\iota(d)=\sum_{j=1}^{q} \frac{d^{j}}{\delta^{j}}=$ $\sum_{j=1}^{q} \frac{b^{j}}{\delta^{j}}-\frac{\tilde{b}^{j}}{\delta^{j}}=\sum_{j=1}^{q}\left|\frac{\tilde{b}^{j}}{\delta^{j}}-\frac{b^{j}}{\delta^{j}}\right|=L_{1}\left(\tilde{b}^{\delta}, b^{\delta}\right)$, where $b^{\delta}=\left(\frac{b^{1}}{\delta^{1}}, \ldots, \frac{b^{q}}{\delta^{q}}\right)$.

In what follows, $\iota$ will be at the core of the formalization of the notion of competition.

\subsubsection{The notion of competition and related issues}

At first, we will define the competition between one offer and a subset of offers.

Definition 23 Let $b \in \mathcal{B}$ and $\mathcal{O} \subseteq \mathcal{B}$. The competition $\gamma(b, \mathcal{O})$ is defined by

\footnotetext{
${ }^{4}$ This information is assumed to be provided by the buyer. No other information about his preferences is requested.
} 


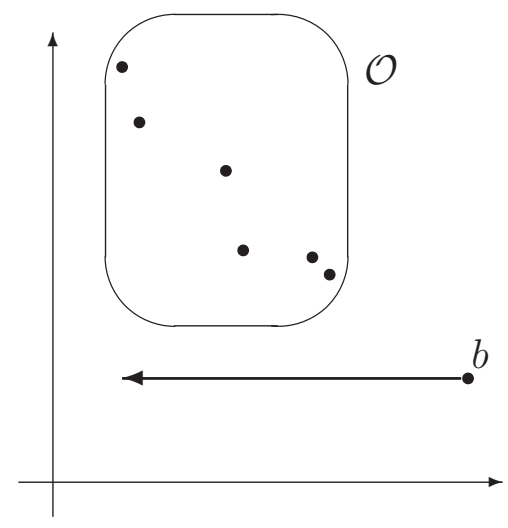

Figure 6.7: The competition of an offer $b$ with respect to a subset of offers $\mathcal{O}$

$\gamma(b, \mathcal{O})=\iota(\{b, i(\{b\} \cup \mathcal{O}))$

Intuitively, $\gamma(b, \mathcal{O})$ quantifies the minimal improvement needed for offer $b$ to dominate all the offers belonging to $\mathcal{O}$. Consequently, if $b$ dominates all the offers in $\mathcal{O}$ then $\gamma(b, \mathcal{O})=0$. The more $\gamma(b, \mathcal{O})$ increases the less $b$ is competitive with respect to the offers in $\mathcal{O}$.

Now that the competition notion has been introduced, we are ready to define what we will call a intra-set competition.

Definition 24 Let $\mathcal{O} \subseteq \mathcal{B}$, we call intra-set competition:

$$
I S(\mathcal{O})=\min _{b \in \mathcal{O}} \gamma(b, \mathcal{O})
$$

In other words, the intra-set competition quantifies the minimum number of improvements that one offer belonging to $\mathcal{O}$ needs to do in order to beat all the other offers of $\mathcal{O}$. Consequently, if $\tilde{b} \in \mathcal{O}$ is such that $\tilde{b} \preceq b, \forall b \in \mathcal{O}$ we have $I S(\mathcal{O})=0$. The more $I S(\mathcal{O})$ increases the less competititive is $\mathcal{O}$.

\subsubsection{IS partitions}

As already stressed, our aim is to partition the set of bids $\mathcal{B}$ into bidding niches. First we will restrict ourselves to cone partitions: 
Definition $25 A k$ cone partition of $\mathcal{B}$, noted $C P_{k}$, is a partition $\left\{C_{1}, \ldots, C_{k}\right\}$ of $\mathcal{B}$ such that $C_{i}=N\left(C_{i}\right)$ where $N(\mathcal{O})=\{b \in \mathcal{B} \mid i(\mathcal{O}) \preceq b\},(\mathcal{O} \subseteq \mathcal{B})$.

Considering cone partitions ensures that the bidding space is divided into non overlapping subsets. In other words, if one offer $b \in C_{i}$ is improved up to exactly dominate all the other offers in $C_{i}$ (i.e. up to $i\left(C_{i}\right)$ ), it will not beat offers outside $C_{i}$. Furthermore, partitioning a subset of $m$ elements leads to a combinatorial explosion of possibilities, known as the Bell number. In our context, restricting ourselves to cone partitions reduces the number of options as shown in section 6.4.2. In what follows, the family of all $\mathrm{k}$ cone partitions will be denoted $\mathcal{C P}_{k}$.

Of course, to each element $C_{i}$ of a k cone partition $C P_{k}$ an intra-set competition $I S\left(C_{i}\right)$ can be assigned. Globally, the competition of the $\mathrm{k}$ cone partition, noted $I S_{\max }\left(C P_{k}\right)$ is evaluated as being the largest intra-set competition of its constitutive elements. More formally;

$$
I S_{\max }\left(C P_{k}\right)=\max _{C_{i} \in C P_{k}} I S\left(C_{i}\right)
$$

This choice is motivated by the fact that a $\mathrm{k}$ cone partition is considered to be competitive (i.e. if $I S_{m} a x\left(\mathcal{C P}_{k}\right)$ is low) only if all the intra-set competitions of its elements are low. To our point of view, when evaluating the intra set competitions of a $\mathrm{k}$ cone partition, a good score of one constitutive element can, in no way, compensate the bad score of another element.

Among all the possible $\mathrm{k}$ cone partitions $C P_{k}$ we will focus on the most competitive ones. These will be referred to IS partitions.

Definition 26 A $k$ cone partition $C P_{k}^{*}$ is said to be a IS partition if:

$$
C P_{k}^{*}=\arg \min _{C P_{k} \in \mathcal{C} \mathcal{P}_{k}} I S_{\max }\left(C P_{k}\right)
$$

\subsubsection{Bidding niches partitions}

So far, given a value for $\mathrm{k}$, a IS partition allows us to split the offers into $\mathrm{k}$ sets such that each of them has a high competition degree. In order to obtain what we call a partition in bidding niches we further require that these sets 
are not too competitive between themselves ${ }^{5}$. To quantify this aspect we introduce the extra-set competition.

Definition 27 Let $\mathcal{O} \subset \mathcal{B}$, we call extra-set competition:

$$
E S(\mathcal{O})=\min _{b \notin \mathcal{O}} \gamma(b, \mathcal{O})
$$

The extra-set competition quantifies the minimum number of improvements that one offer outside $\mathcal{O}$ needs to do in order to beat all the offers of $\mathcal{O}$. Consequently, if $\tilde{b} \notin \mathcal{O}$ is such that $\tilde{b} \preceq b, \forall b \in \mathcal{O}$ we have $E S(\mathcal{O})=0$. The more $E S(\mathcal{O})$ increases, the less $\mathcal{O}$ is likely to be beaten from a offer $b \notin \mathcal{O}$. Finally, let us stress that $E S$ is not defined for the entire set of offers $\mathcal{B}$.

In the same way $I S_{\max }$ has been introduced, we will globally characterize the extra-set competition of the elements of a given IS partition, noted $E S_{\min }\left(C P_{k}^{*}\right)$ as follows:

$$
E S_{\min }\left(C P_{k}^{*}\right)=\min _{C_{i} \in C P_{k}} E S\left(C_{i}\right)
$$

This choice is motivated by the fact that a IS partition is considered to be a partition in bidding niches only if all the extra set competitions of its constitutive elements are low.

Among all the IS partitions (for $k=2, \ldots, m$ ) we will retain the one with, simultaneously, a high $E S_{\min }$ and a low $I S_{\max }$. These two objectives can be aggregated to denote the quality of a given IS partition $Q\left(C P_{k}^{*}\right)=$ $E S_{\min }\left(C P_{k}^{*}\right)-I S_{\max }\left(C P_{k}^{*}\right)$. Therefore, what we call a partition of $B$ into bidding niches, noted $B N P$, will be defined as follows:

$$
B N P=\arg \max _{k=2 \ldots, m}\left\{E S_{\min }\left(C P_{k}^{*}\right)-I S_{\max }\left(C P_{k}^{*}\right)\right\}
$$

\footnotetext{
${ }^{5}$ these considerations can be related to traditional classification problems where the aim is to find homogenous groups that are heterogeneous between themselves.
} 


\subsection{Hierarchical algorithm}

The total number of cone partitions that can be built, for a given number of criteria and a given number of offers, is such that, in most cases, a complete enumeration is impossible (see section 6.4.2). Therefore, heuristics are needed to find bidding niches partitions. Among them, we have adopted a hierarchical scheme presented here after (see algorithm 5).

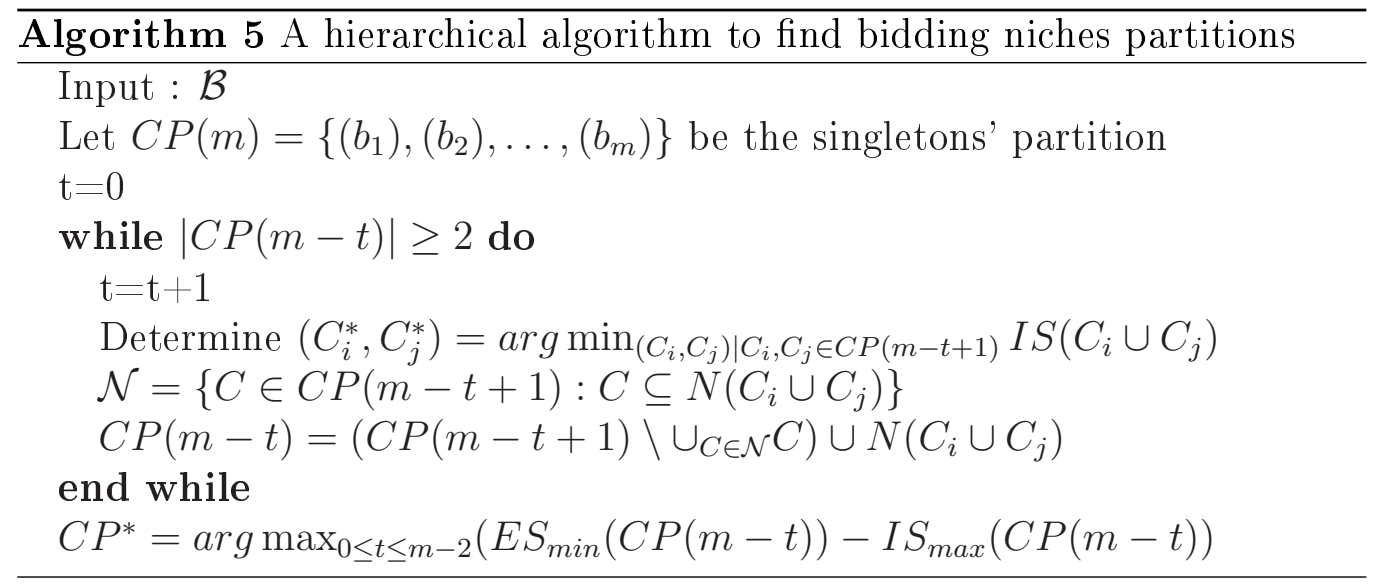

At each step of the algorithm, two niches are merged such that the worst intra-set competition of the resulting partition is minimal. Starting with the partition where each offer is considered to form a niche on its own, the number of niches of the partition is then iteratively reduced.

Of course, even if the simplicity of hierarchical approaches is appealing, a number bottlenecks have been stressed in the literature. Among them, once two niches have been merged, they can never be split again. Hence, a locally optimal merge may mislead the algorithm.

In the present context, another distinctive feature needs to be stressed; the cone obtained by merging two niches $N_{i}$ and $N_{j}$ of a partition $C P(t-$ 1) may contain other niches. For instance, consider the three offers $b_{1}=$ $(1,0,0), b_{2}=(0,1,0)$ and $b_{3}=(0,0,1)$. By merging the two offers $b_{1}$ and $b_{2}$ the third offer $b_{3}$ belongs to the cone formed by the two first offers. Since, at each step of the algorithm, we want to have a feasable solution, i.e. a cone partition, $C P$ will consist, on the one hand, of the cone $N\left(N_{i} \cup N_{j}\right)$ obtained by merging $N_{i}$ and $N_{j}$ and, on the other hand, of the remaining niches $N_{p}$, where $N_{p} \in C P(t-1)$, but $N_{k} \nsubseteq N\left(N_{i} \cup N_{j}\right)$. For this reason, the hierarchical algorithm will not necessarily find a partition into $k$ bidding niches for each $k=2, \ldots, m$. 
In practice, the buyer often wants to obtain a partition with only a few niches. Therefore, the last constraint can be adapted in order to restrict the selection among the partitions with at most $m$ niches. In what follows, we will assume that the buyer further specifies that the number of niches in the partition is lower than or equal to $q+2$ (which is, to our point of view, a reasonable choice). On the other hand, one can imagine that the buyer may want to obtain a partition characterized by a fixed number of $M$ niches. In this case, the last step is not necessary anymore and the solution is directly available among those that have been iteratively built.

\subsection{Empirical tests}

This section is devoted to the empirical analysis of the model and the algorithm proposed in the previous sections. More precisely, we will investigate:

1. the average number of cone partitions that can be enumerated for a given number of criteria and offers (in section 6.4.2);

2. the assignments provided by our algorithm when applied on artificial data sets (in section 6.4.3);

3 . the assignment's sensitivity with respect to the value of the $\delta$ parameters (in section 6.4.4);

4. the assignment's sensitivity with respect to the improvement of several offers (in section 6.4.5);

5. and, finally, the difference between simultaneous versus distinct management of bidding niches (in section 6.4.6).

Therefore, a number of assumptions have been considered (see table 6.1 for a global perspective):

1. the number of criteria, $q$, could vary from 2 to 5 ; to our point of view, considering more criteria is, in practice, artificial.

2. the number of niches, $k$, could vary from 2 to $q+1$; limiting the maximum number of niches with respect to the number of criteria was, to our perspective, a reasonable assumption. 
3. the number of observations in a given niche is equal to 5 and all the niches contain the same number of observations. Of course, this assumption may open criticism. In preliminary tests, we have rather used a random number of offers given by $q+\lfloor 4 \cdot \mathcal{U} \cdot q\rceil$ where $\mathcal{U} \sim U_{[0,1]}$ but the results were more difficult to interpret. Therefore, for clarity and simplicity reasons, we have decided to use the same number of offers whatever the number of criteria and niches. The arbitrary value of 5 offers per niche was, according to us, a reasonable choice. Indeed, choosing less offers was not representative. If the bidding space only contains 4 offers (i.e. for instance 2 'niches' containing 2 offers each) there is few need of analyzing it in terms of bidding niches. On the contrary, if more offers were chosen the total number of bids in the bidding could explode leading to an unrealistic situation (5 niches containing 15 offers each lead to a total of 75 offers).

\begin{tabular}{ccc}
\hline nb criteria & nb niches & total number offers \\
\hline 2 & 2 & 10 \\
2 & 3 & 15 \\
\hline 3 & 2 & 10 \\
3 & 3 & 15 \\
3 & 4 & 20 \\
\hline 4 & 2 & 10 \\
4 & 3 & 15 \\
4 & 4 & 20 \\
4 & 5 & 25 \\
\hline 5 & 2 & 10 \\
5 & 3 & 15 \\
5 & 4 & 20 \\
5 & 5 & 25 \\
5 & 6 & 30 \\
\hline
\end{tabular}

Table 6.1: Structure of the tests performed.

In what follows, we will often need to compare partitions. Therefore, we have chosen to use the Rand index (88). Intuitively, the Rand index between two partitions $P$ and $Q$, noted $R(P, Q)$, is the percentage of pairs for which there is an agreement. More formally, let $A(P)$ be the assignment matrix associated to a partition $P^{6}$, the $R(P, Q)$ is given by:

\footnotetext{
${ }^{6}$ i.e. $A(P)_{i j}=1$ is $b_{i}$ and $b_{j}$ are assigned to the same subset in $P, A(P)_{i j}=0$ if $b_{i}$ and $b_{j}$ are assigned to different subsets in $P$
} 


$$
R(P, Q)=\frac{|A(P) \wedge A(Q)|-n}{\frac{n \cdot(n-1)}{2}}
$$

Obviously, if $R(P, Q)=1$ the two partitions are identical. On the other hand, when assignment differences exist between $P$ and $Q$, we have $R(P, Q)<1$. Several authors have criticized the use of the Rand index ${ }^{7}$ and other measures such as the Jaccard index, the Wallace index or the normalized Lerman index are also commonly used in the literature. Nevertheless, the simplicity of the Rand index has retained our attention. Due to its nature, the interpretation of its value will, at first, be limited to the higher the better. Additionally, in order to further enrich our results, we will test the hypothesis $H 0$ of random assignments. Therefore, we have empirically estimated the $95 \%$ percentile Rand index between two random partitions for a given number of observations (see appendix 7 for details about the algorithm and the related values).

To study questions related to sections $6.4 .3,6.4 .4$ and 6.4 .5 , one need to generate data sets describing bidding niches. This phase is not obvious and, therefore, is commented in section 6.4.1.

\subsubsection{A few words about the generation of artificial data sets}

Following the model introduced in section 6.2 , creating a bidding niches partition imposes to create a data set and a related assignment that is optimal for the quality criterion. Until now, we haven't found a way to perform this task. Therefore, we will limit ourselves to data sets generated as explained here after. To our point of view, these examples constitute good candidates to test our model and algorithm (even tough we have no guarantee about the optimality of the assignment). As a consequence, the application of the hierarchical algorithm will, sometimes, lead to a better assignment than the one initially proposed.

Basically, the artificial data sets generation rely on two assumptions:

1. the bidding niches are created successively such that the ideal point related to the $m^{\text {th }}$ bidding niche, noted $i(N(m))$, is not dominated and does not dominate the ideal points related to the niches that have

\footnotetext{
${ }^{7}$ since it is not equal to zero for two random partitions
} 
already been created. Furthermore, the vector $i(N(m))-i(N(m-1))$ contains only two non-null elements, the value of which are respectively $\alpha$ and $-\alpha$ and the positions (in the vector) of which are random (as a consequence, the $L_{1}$ distance between two succession ideal points is equal to $2 \cdot \alpha$ )

2. once the ideal point of every niche has been determined, the pareto optimal bids within each niche are generated in such a way that their $L_{1}$ distance to the ideal point of the niche is distributed according to $U_{[0,1]}$.

Figure 6.8 illustrates such a data set. As a consequence of the previous assumptions, for all niches $N$, we have $I S(N) \leq 1^{8}$ and $E S(N) \geq \alpha$. As soon of $\alpha \geq 1$ no overlapping between the niches can happen. In what follows, we will both use the value $\alpha=1$ and $\alpha=0.75$. The latter expresses cases where overlappings between niches may occur. See table 6.2 for illustration.

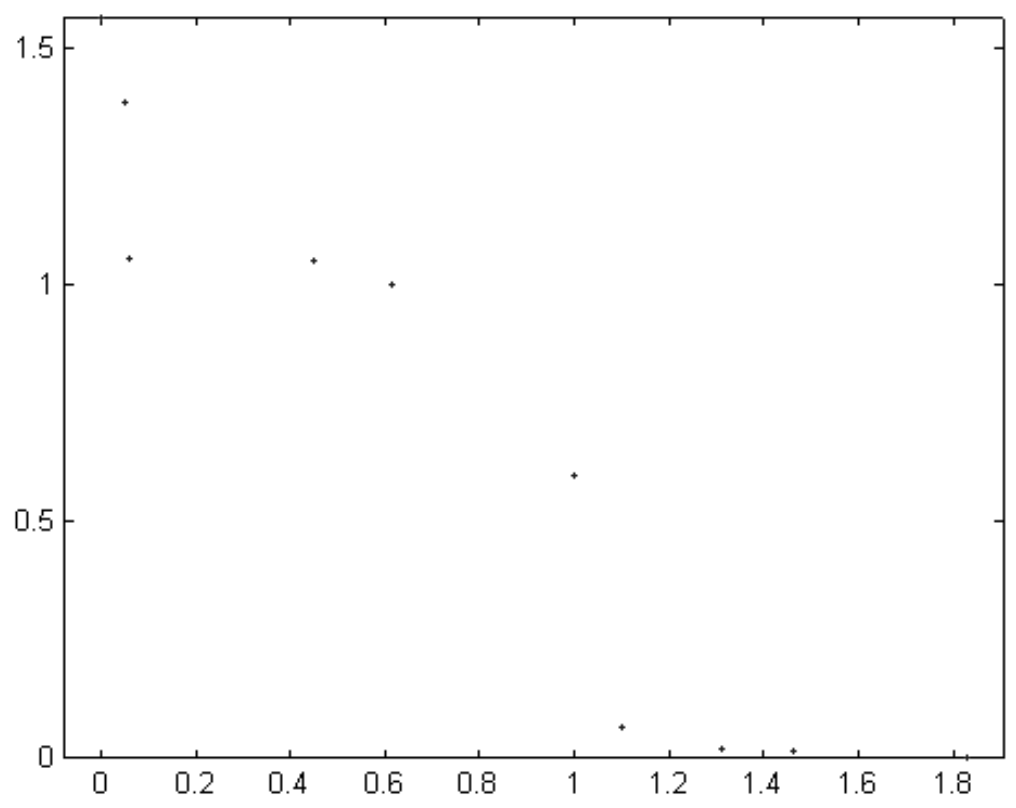

Figure 6.8: Example of a data set with 2 niches of 5 observations.

\footnotetext{
${ }^{8}$ Moreover as a consequence of uniform distribution of the distances to the ideal point we can state that $I S(N) \sim n \cdot\left(1-F_{U}\right)^{(n-1)} \cdot f_{U}$
} 


\begin{tabular}{|c|c|cc||cc|}
\hline & & \multicolumn{2}{|c||}{5 obs. } & \multicolumn{2}{c|}{7 obs. } \\
nb. crit. & nb. niches & Min. Dist. & Overlapping & Min. Dist. & Overlapping \\
\hline 2 & 2 & 0,0141 & $79,80 \%$ & 0,0035 & $92,00 \%$ \\
2 & 3 & 0,0028 & $95,00 \%$ & 0,0008 & $99,00 \%$ \\
3 & 2 & 0,2548 & $4,80 \%$ & 0,2007 & $6,40 \%$ \\
3 & 3 & 0,1598 & $9,60 \%$ & 0,1127 & $17,40 \%$ \\
3 & 4 & 0,1162 & $15,20 \%$ & 0,0781 & $22,60 \%$ \\
4 & 2 & 0,399 & $0,00 \%$ & 0,3561 & $0,20 \%$ \\
4 & 3 & 0,3247 & $0,60 \%$ & 0,2804 & $0,40 \%$ \\
4 & 4 & 0,281 & $0,40 \%$ & 0,2455 & $0,00 \%$ \\
4 & 5 & 0,2483 & $1,60 \%$ & 0,2097 & $1,00 \%$ \\
5 & 2 & 0,4842 & $0,00 \%$ & 0,4618 & $0,00 \%$ \\
5 & 3 & 0,4249 & $0,00 \%$ & 0,3919 & $0,00 \%$ \\
5 & 4 & 0,3878 & $0,00 \%$ & 0,3626 & $0,00 \%$ \\
5 & 5 & 0,3656 & $0,00 \%$ & 0,3357 & $0,00 \%$ \\
5 & 6 & 0,3546 & $0,00 \%$ & 0,3205 & $0,00 \%$ \\
\hline
\end{tabular}

Table 6.2: Minimal distance between two niches and percentage of overlapping niches for data sets generated with $\alpha=0.75$

\subsubsection{Estimation of the number of cone partitions}

Partitioning a set of $n$ elements into $k$ subsets leads to a combinatorial number of possibilities. In the literature, this is referred as the Stirling number of the second kind, noted $S(n, k)$, while the Bell number, noted $B(n)$, represents the total number of potential partitions ${ }^{9}$. In the present work, we restrict ourselves to cone partitions, i.e. a subset of all the potential partitions. Therefore, a natural question is to wonder how many cone partitions can be built for a given number of elements. This will be at the core of the present section.

At first, let us remark that, as a consequence of the cone partitions' definition, their total number, noted $N C P(n, q)$, is likely to be influenced both by the number of pareto optimal elements and by the related number of criteria. Morevover, when only 2 criteria are considered, we have $\operatorname{NCP}(n, 2)=2^{(n-1)}$.

Table 6.3 presents the average number of cone partitions, noted $N \bar{C} P$, for different combinations of $q$ and $n$. These statistics results from explicit

${ }^{9}$ whatever the number of subsets 
enumeration ${ }^{10}$. For each instance of the parameters, 1.000 instances have been generated. Additionally, these results have been normalized by the related Bell number. The last three columns show that the average number of cone partitions increases slower than the Bell number.

\begin{tabular}{cccccccc}
\hline $\mathrm{n}$ & $B(n)$ & $N \bar{C} P(n, 3)$ & $N \bar{C} P(n, 4)$ & $N \bar{C} P(n, 5)$ & $\frac{N C P(n, 3)}{\mathcal{B}(n)}$ & $\frac{N C P(n, 4)}{\mathcal{B}(n)}$ & $\frac{N C P(n, 5)}{\mathcal{B}(n)}$ \\
\hline 2 & 2 & 2 & 2 & 2 & $100,00 \%$ & $100,00 \%$ & $100,00 \%$ \\
3 & 5 & 4,48 & 4,69 & 4,7 & $89,60 \%$ & $93,80 \%$ & $94,00 \%$ \\
4 & 15 & 10,22 & 11,54 & 12,15 & $68,13 \%$ & $76,93 \%$ & $81,00 \%$ \\
5 & 52 & 10,22 & 11,54 & 12,15 & $19,65 \%$ & $22,19 \%$ & $23,37 \%$ \\
6 & 203 & 54,85 & 77,98 & 98,25 & $27,02 \%$ & $38,41 \%$ & $48,40 \%$ \\
7 & 877 & 127,85 & 213,88 & 275,05 & $14,58 \%$ & $24,39 \%$ & $31,36 \%$ \\
8 & 4140 & 311,86 & 601,63 & 1023,75 & $7,53 \%$ & $14,53 \%$ & $24,73 \%$ \\
9 & 21147 & 695,51 & 1551,3 & 2690,93 & $3,29 \%$ & $7,34 \%$ & $12,72 \%$ \\
10 & 115975 & 1762,5 & 4591,95 & 8972,96 & $1,52 \%$ & $3,96 \%$ & $7,74 \%$ \\
\hline
\end{tabular}

Table 6.3: Average number of cone partitions for different combinations of the number of observations and criteria.

To further explore how $N \bar{C} P(n, q)$ evolves with respect to $n$ (for a given $q$ ), we have tried to fit the data according to the following model $N \bar{C} P(n, q)=$ $a^{n} \cdot b$. Table 6.4 summarizes the results obtained for the different values of $q$.

\begin{tabular}{cccc}
\hline$q$ & $a$ & $b$ & $R^{2}$ \\
\hline 2 & 2 & 0.5 & 1 \\
3 & 2.331599 & 0.350902 & 0.999786 \\
4 & 2.63902 & 0.251895 & 0.999178 \\
5 & 2.887754 & 0.192029 & 0.997897 \\
\hline
\end{tabular}

Table 6.4: Parameters of model $N \bar{C} P=a^{n} \cdot b$.

Finally, the following model ${ }^{11}$ gives a rough idea about the global behavior of $N \bar{C} P(n, q)$.

$$
N \bar{C} P(n, q)=\frac{(2+\log 2 \cdot(q-2))^{n}}{q}
$$

\footnotetext{
${ }^{10}$ which was still reasonable since the number of elements was lower than 10 . Pareto optimal observations were generated using a uniform distribution.

${ }^{11} R^{2}=0.995394$
} 


\subsubsection{Testing the hierarchical algorithm on artificial data sets}

In this section, we investigate the assignments provided by the hierarchical algorithm when applied on artificial data sets (see section 6.4.1). In addition to the benchmark configuration, i.e. $n=5$ and $\alpha=1$, we have ran tests for $n=7$ and $\alpha=0.75$. For every considered combination $(\alpha, n, q)$ we have simulated 500 iterations. Related results are listed in table 6.5 and 6.6.

First of all, let us stress that, in a number of cases, the hierarchical algorithm has stopped with a quality index higher than the quality index of the initial data set (see row $Q_{o b s} \geq Q_{t h}$ in table 6.5 and 6.6). As already stressed, this effect is due to the fact that it is not easy to build a data set and a related assignment that is optimal for the quality criterion. The way the test sets have been generated does not guarantee that another assignment could no lead to a better quality. In these cases, the comparison of the hierarchical assignment and the initial one is not meaningful. Therefore, these instances will not be taken into account in the average Rand index.

Let us note that for data sets where $Q_{o b s} \geq Q_{t h}$, the HO hypothesis, that the Rand index between the initial partition and the one found by the hierarchical algorithm is random, is always rejected. This is not surprising, given the average Rand indexes listed in table 6.5 and 6.6 , which show that the assignments are similar whatever the values $n, q, \alpha$. As soon as the number of criteria is greater of equal to 3 these statistics are excellent. When only 2 criteria are considered, the results remains good but are less striking. This is due to the fact that the number of bids is big regarding the number of criteria leading to a higher bids'density.

In order to further characterize the behavior of the rand index with respect to the couples $(n, q)$ we have limited ourselves to 2 niches and 5,7 and 10 bids per niches. See results in table 6.7.

\subsubsection{Testing the assignment'sensitivity with respect to the $\delta$ parameters}

As already stressed in section 6.2 , the normalization role of the $\delta$ parameters is essential. Indeed, it allows to manage the improvements on different criteria characterized by different units. 


\begin{tabular}{|c|c|cc||cc|}
\hline \multicolumn{6}{|c|}{$\alpha=1$} \\
\hline \multirow{2}{*}{ nb. crit. } & nb. niches & \multicolumn{2}{|c|}{5 obs. } & \multicolumn{2}{c|}{7 obs. } \\
\hline 2 & 2 & 0,963 & $12,20 \%$ & 0,9769 & $11,20 \%$ \\
2 & 3 & 0,9591 & $15,00 \%$ & 0,9722 & $8,80 \%$ \\
3 & 2 & 0,9972 & $0,40 \%$ & 0,9974 & $0,40 \%$ \\
3 & 3 & 0,9934 & $2,00 \%$ & 0,9976 & $1,80 \%$ \\
3 & 4 & 0,9928 & $37,20 \%$ & 0,9967 & $34,40 \%$ \\
4 & 2 & 1 & $0,00 \%$ & 0,9997 & $0,00 \%$ \\
4 & 3 & 0,9998 & $0,20 \%$ & 0,9998 & $0,20 \%$ \\
4 & 4 & 0,9984 & $44,20 \%$ & 0,9992 & $46,20 \%$ \\
4 & 5 & 0,9993 & $18,00 \%$ & 0,9998 & $17,80 \%$ \\
5 & 2 & 1 & $0,00 \%$ & 1 & $0,00 \%$ \\
5 & 3 & 0,9998 & $0,60 \%$ & 1 & $0,00 \%$ \\
5 & 4 & 0,9998 & $45,60 \%$ & 1 & $47,00 \%$ \\
5 & 5 & 0,9992 & $17,00 \%$ & 1 & $14,80 \%$ \\
5 & 6 & 0,9998 & $36,40 \%$ & 1 & $35,00 \%$ \\
\hline
\end{tabular}

Table 6.5: Average $R$ and percentage of cases with a lower or equal quality than the expected quality for data sets with $\alpha=1$ 


\begin{tabular}{|c|c|cc||cc|}
\hline \multicolumn{5}{|c|}{$\alpha=0.75$} \\
\hline \multirow{2}{*}{ nb. crit. } & nb. niches & \multicolumn{2}{|c|}{5 obs. } & \multicolumn{2}{c|}{7 obs. } \\
\hline 2 & 2 & 0,8376 & $63,40 \%$ & 0,8426 & $72,40 \%$ \\
2 & 3 & 0,8279 & $57,00 \%$ & 0,8238 & $55,00 \%$ \\
3 & 2 & 0,9797 & $11,00 \%$ & 0,9858 & $14,00 \%$ \\
3 & 3 & 0,9773 & $20,20 \%$ & 0,9757 & $22,20 \%$ \\
3 & 4 & 0,9683 & $51,20 \%$ & 0,9698 & $53,40 \%$ \\
4 & 2 & 0,9978 & $3,20 \%$ & 0,9981 & $2,00 \%$ \\
4 & 3 & 0,997 & $6,80 \%$ & 0,9968 & $4,20 \%$ \\
4 & 4 & 0,989 & $46,60 \%$ & 0,9964 & $45,80 \%$ \\
4 & 5 & 0,993 & $25,60 \%$ & 0,9955 & $23,60 \%$ \\
5 & 2 & 0,9977 & $0,60 \%$ & 1 & $0,60 \%$ \\
5 & 3 & 0,9985 & $3,60 \%$ & 0,9998 & $2,00 \%$ \\
5 & 4 & 0,9991 & $52,60 \%$ & 0,9992 & $46,60 \%$ \\
5 & 5 & 0,9997 & $19,60 \%$ & 0,9995 & $16,40 \%$ \\
5 & 6 & 0,9979 & $38,60 \%$ & 0,9998 & $39,40 \%$ \\
\hline
\end{tabular}

Table 6.6: Average $R$ and percentage of cases with a lower or equal quality than the expected quality for data sets with $\alpha=0.75$

\begin{tabular}{cccc}
\hline nb. crit. & 5 obs. & 7 obs. & 10 obs. \\
\hline 2 & 0.963 & 0.9769 & 0.9786 \\
3 & 0.9972 & 0.9974 & 0.9992 \\
4 & 1 & 0.9997 & 0.9996 \\
5 & 1 & 1 & 1 \\
\hline
\end{tabular}

Table 6.7: $R$ average for 2 niches. 
Of course, a natural question that arises is to wonder wether the assignments provided by our approach are very sensible to these variations. Therefore, we have conducted simulations the results of which are summarized in table 6.8. These tests follow the scheme described here after (see algorithm $6)$. For every combination of the number of niches $n$ and the number of criteria $q$, we have ran 500 tests.

At first, i.e. when $T S(n, q)$ is generated, all the $\delta^{j}$ parameters are assumed to be equal to one. Then, these parameters are perturbed such that $\delta^{j}=$ $1-e+e \cdot \mathcal{U}$ where $\mathcal{U} \sim U_{[0,1]}$. Two values of $e$ are considered in the tests; either $e=0.1$, either $e=0.2$. As a consequence, when $e=0.1$ (resp. $e=0.2$ ) we have $\frac{\delta^{j}}{\delta^{i}} \in\left[\begin{array}{ll}0.818 & 1.222\end{array}\right]$ (resp. [0.666 1.5]). In other words, a maximal relative error ${ }^{12}$ of, approximately, $40 \%$ (resp. $85 \%$ ) is accepted between any pairs of $\delta$ parameters. This constraint allows us to model hesitations of the buyer about the values of $\delta$.

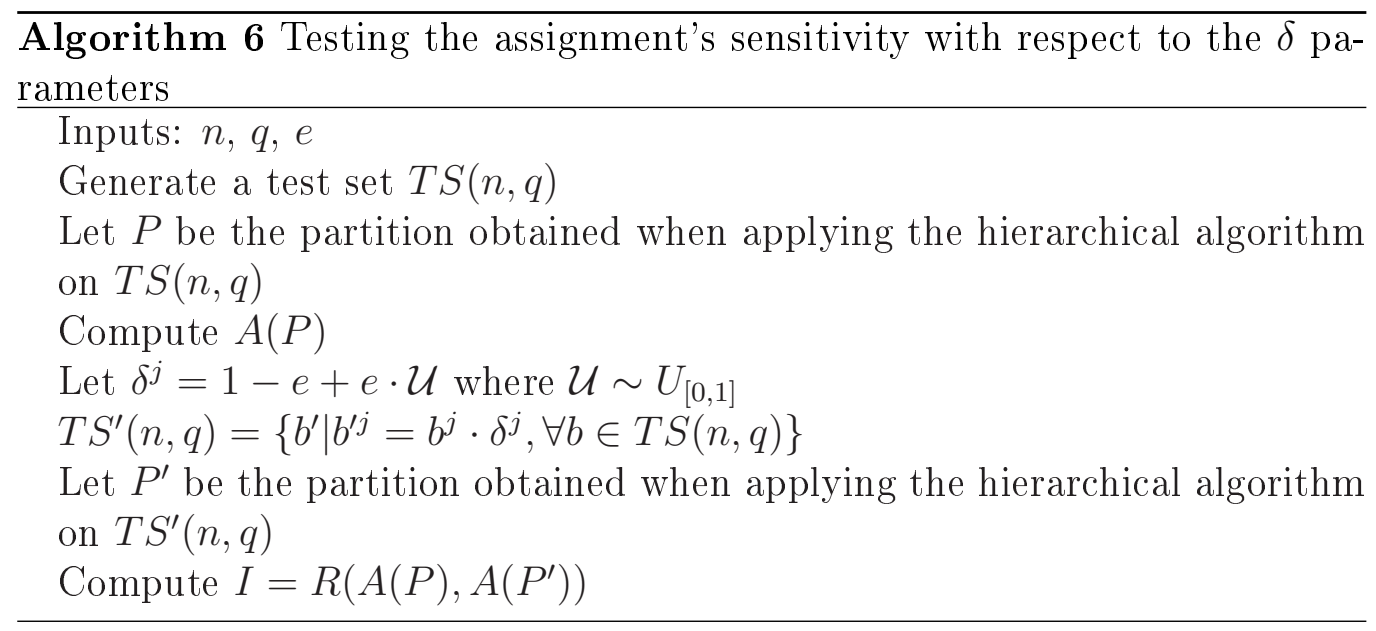

As shown in table 6.8, assignments provided by the algorithm are not too sensible to reasonable variations of the parameters $\delta$. Moreover, the number of cases where the comparison of the ex-ante and ex-post assignments can be considered as random is limited.

\subsubsection{Testing the assignment's sensitivity with respect to the improvement of offers}

Up to now, our analyzes have been limited to a static context while, during an auction, offers are regularly improved. In this section, we empiri-

\footnotetext{
${ }^{12}$ over estimating or under estimating
} 


\begin{tabular}{|c|c|cc||cc|}
\hline & & \multicolumn{2}{|c||}{$\mathrm{e}=10 \%$} & \multicolumn{2}{c|}{$\mathrm{e}=20 \%$} \\
\hline nb. crit. & nb. niches & $R$ average & H0 accepted & $R$ average & HO accepted \\
\hline 2 & 2 & 0,9952 & $0,80 \%$ & 0,9902 & $0,80 \%$ \\
2 & 3 & 0,9962 & $0,20 \%$ & 0,9913 & $0,00 \%$ \\
3 & 2 & 0,9935 & $0,00 \%$ & 0,998 & $0,00 \%$ \\
3 & 3 & 0,9952 & $0,20 \%$ & 0,9971 & $0,00 \%$ \\
3 & 4 & 0,9582 & $0,60 \%$ & 0,934 & $0,60 \%$ \\
4 & 2 & 0,9968 & $0,00 \%$ & 0,9996 & $0,00 \%$ \\
4 & 3 & 0,999 & $0,00 \%$ & 0,9936 & $0,00 \%$ \\
4 & 4 & 0,9316 & $1,60 \%$ & 0,933 & $1,60 \%$ \\
4 & 5 & 0,9618 & $0,40 \%$ & 0,9509 & $0,40 \%$ \\
5 & 2 & 0,9988 & $0,00 \%$ & 1 & $0,00 \%$ \\
5 & 3 & 0,9961 & $0,00 \%$ & 0,9986 & $0,00 \%$ \\
5 & 4 & 0,922 & $3,00 \%$ & 0,9239 & $3,00 \%$ \\
5 & 5 & 0,9743 & $0,40 \%$ & 0,9649 & $0,40 \%$ \\
5 & 6 & 0,935 & $1,60 \%$ & 0,932 & $2,20 \%$ \\
\hline
\end{tabular}

Table 6.8: Sensitivity analysis for data sets with $\alpha=1$ and 5 observations

cally study the impact of such improvements on the assignment provided by the hierarchical algorithm. The tests we have performed follow the scheme described in algorithm 7. The related results are listed in tables 6.9 and 6.10.

Of course, there are many ways to model the improvements of offers. First of all, not all the offers are improved simultaneously. In what follows, an offer $b$ is improved (respectively not improved) with a probability $p$ (respectively $1-p$ ). In this case, the minimal improvement in order to dominate (at least) another offer is applied to $b$. Figure 6.9 illustrate such improvement. In the following tests, two values of $p$ have been considered; either $p=0.2$ (on average 1 bid per niche is improved) either $p=0.4$ (on average two bids per niche are improved).

Table 6.9 and 6.10 show that, on average, assignments do not vary sensibly as a consequence of bid improvements. The column labeled $R==1$ specifies the percentage of cases where the assignment is the same before and after the improvements. On the other hand, as show on the last column, in particular cases, they can lead to very different assignments. 


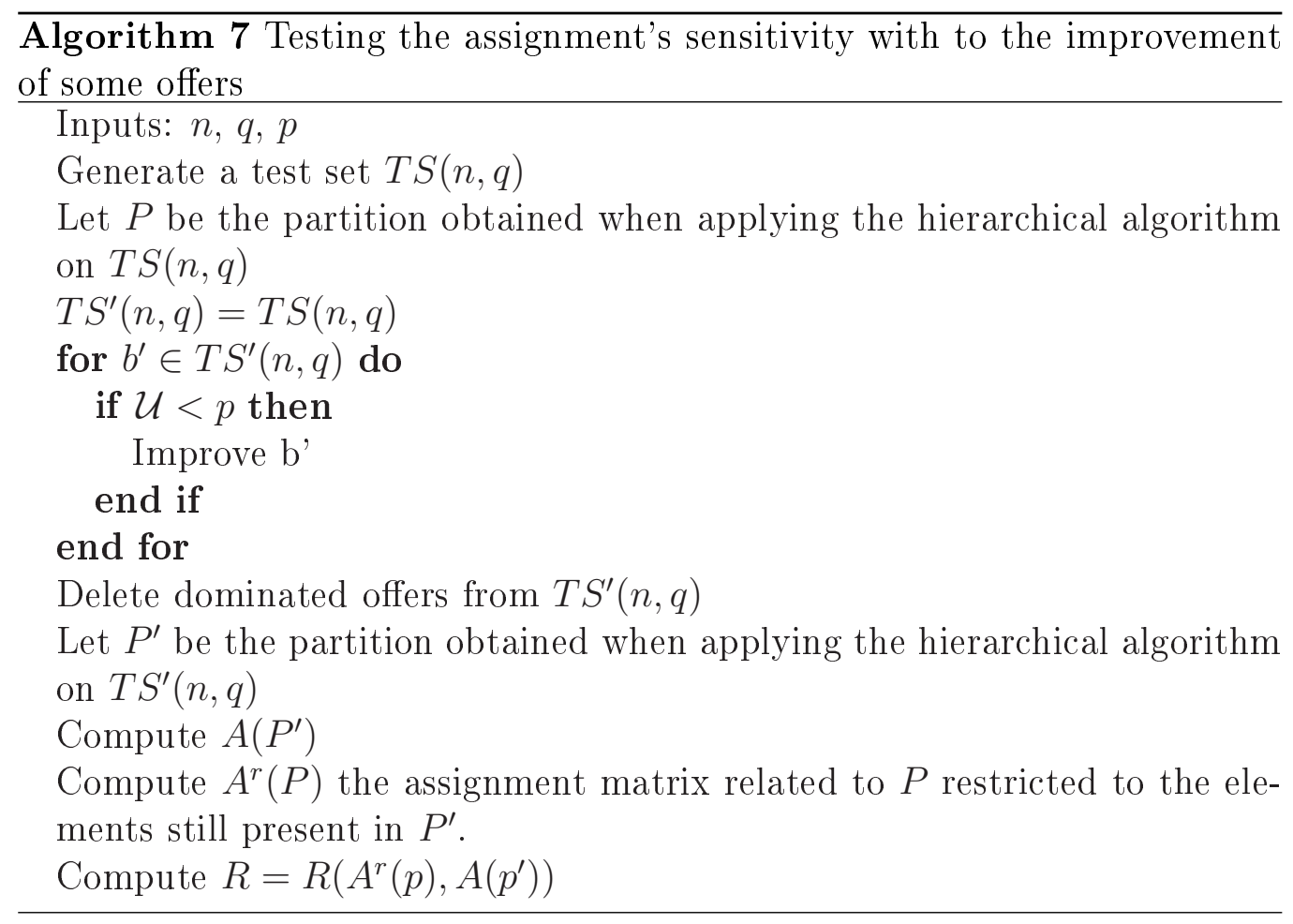

\begin{tabular}{|c|c|ccc|}
\hline \multicolumn{5}{|c|}{$p=0.2$} \\
\hline nb. crit. & nb. niches & $R$ average & $R==1$ & $\min R$ \\
\hline 2 & 2 & 0,972 & $88,20 \%$ & 0,6667 \\
2 & 3 & 0,9761 & $79,60 \%$ & 0,6727 \\
3 & 2 & 0,993 & $97,00 \%$ & 0,7143 \\
3 & 3 & 0,9955 & $96,60 \%$ & 0,7091 \\
3 & 4 & 0,9891 & $89,60 \%$ & 0,675 \\
4 & 2 & 0,9955 & $99,60 \%$ & 0,85713 \\
4 & 3 & 0,9985 & $98,80 \%$ & 0,7802 \\
4 & 4 & 0,992 & $96,60 \%$ & 0,4667 \\
4 & 5 & 0,9948 & $98,00 \%$ & 0,655 \\
5 & 2 & 0,995 & $99,80 \%$ & 0,75 \\
5 & 3 & 0,996 & $99,80 \%$ & 0,7818 \\
5 & 4 & 0,9946 & $97,80 \%$ & 0,7238 \\
5 & 5 & 0,9958 & $98,40 \%$ & 0,6147 \\
5 & 6 & 0,9931 & $97,00 \%$ & 0,6449 \\
\hline
\end{tabular}

Table 6.9: Dynamic evolution $(p=0.2)$ for data sets with $\alpha=1$ and 5 observations 


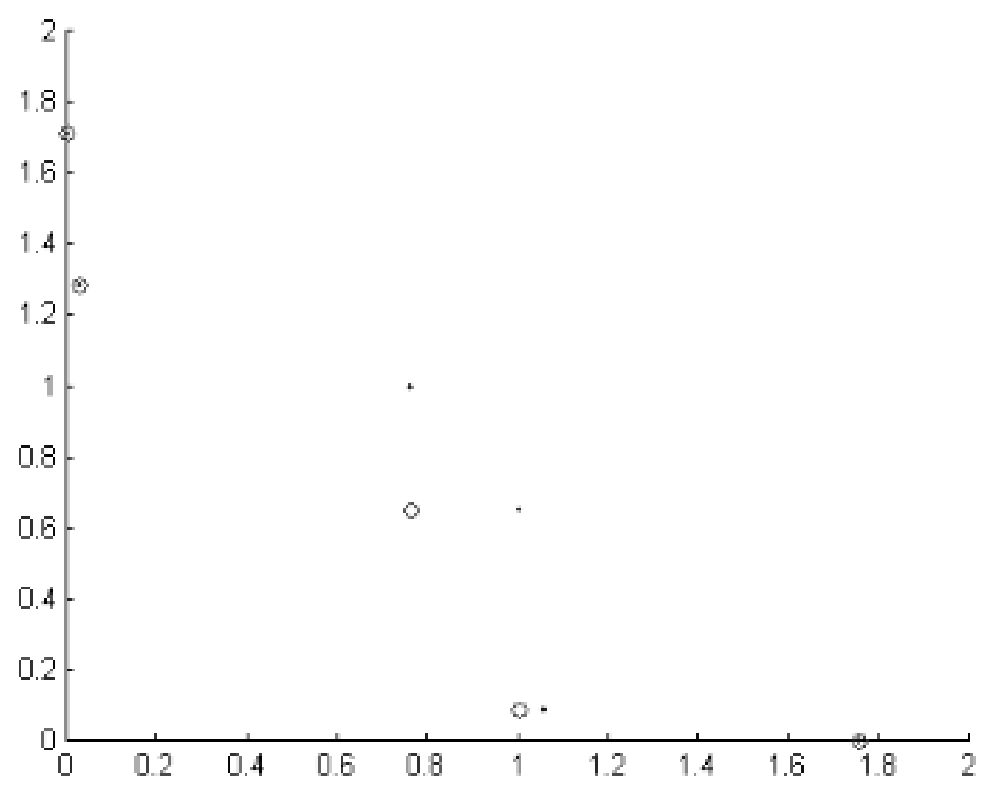

Figure 6.9: Example of offers'improvement. Initial offers are represented by dots.

\subsubsection{Simultaneous versus distinct management of bid- ding niches}

The three examples presented in the introduction of this chapter have showed that the bidding space is not always homogeneous. In particular cases such as, for instance, in the transport problem, the presence of distinct bidding areas was expected a priori ${ }^{13}$. Therefore, one could have decided to organize two distinct multicriteria auctions; one related to the transport by trucks and the other related to the transport by barge, instead of a common multicriteria auction. Afterwards, the best final offer could be selected between the two winning bids (related to these distinct auctions).

First of all, considering distinct auctions (instead of a common one) increases the bidding complexity. Indeed, if the auctions are conducted sequentially, bidders have to continuously evaluate their winning probability in subsequent auctions. Moreover the procurement is likely to last longer than in a simultaneous multicriteria auction. On the other hand, if the auc-

\footnotetext{
${ }^{13}$ on the other hand, such assertion was not obvious for the two other examples
} 


\begin{tabular}{|c|c|ccc|}
\hline \multicolumn{5}{|c|}{$p=0.4$} \\
\hline nb. crit. & nb. niches & $I$ average & $I==1$ & $\min R$ \\
\hline 2 & 2 & 0,9629 & $85,80 \%$ & 0,6 \\
2 & 3 & 0,9572 & $69,60 \%$ & 0,6071 \\
3 & 2 & 0,9885 & $95,20 \%$ & 0,6 \\
3 & 3 & 0,9882 & $90,20 \%$ & 0,697 \\
3 & 4 & 0,966 & $80,80 \%$ & 0,6364 \\
4 & 2 & 1 & $100,00 \%$ & 1 \\
4 & 3 & 0,9984 & $98,80 \%$ & 0,7333 \\
4 & 4 & 0,9824 & $93,80 \%$ & 0,4546 \\
4 & 5 & 0,9867 & $93,80 \%$ & 0,5167 \\
5 & 2 & 1 & $100,00 \%$ & 1 \\
5 & 3 & 0,998 & $99,00 \%$ & 0,7436 \\
5 & 4 & 0,9882 & $95,80 \%$ & 0,4667 \\
5 & 5 & 0,9967 & $98,80 \%$ & 0,6491 \\
5 & 6 & 0,9854 & $94,00 \%$ & 0,615 \\
\hline
\end{tabular}

Table 6.10: Dynamic evolution $(p=0.4)$ for data sets with $\alpha=1$ and 5 observations

tions are conducted in parallel, the bidders have to be present simultaneously in several sessions.

Last but not least, a consequence of conducting several auctions instead of a common one, is a competition decrease. In order to quantify this effect, we have ran simulations the results of which are listed in tables 6.11 and 6.12. Basically, the competition decrease was measured in terms of the ratio between the best utility among the best utilities obtained within each single niches and the best utility for the global set of bids. In other words;

$$
\frac{\min _{i=1, \ldots, k}\left(\min _{b \in C_{i}} U(b)\right)}{\min _{b \in \mathcal{B}} U(b}
$$

Therefore, a traditional additive utility was used: $U(b)=\sum_{j=1}^{q} w^{j} \cdot b^{j}$ (the weights $w^{j}$ being randomly generated, at each simulation, using a uniform distribution). For each combination of the number of criteria and the number of niches, 5.000 iterations have been executed. 


\begin{tabular}{ccccc}
\hline Nb criteria & Nb niches & Same utility \% & $\begin{array}{c}\text { Avg comp. decrease } \\
\text { (different utilities) } \%\end{array}$ & $\begin{array}{c}\text { max comp. } \\
\text { decrease } \%\end{array}$ \\
\hline 2 & 2 & 87,08 & 7,54 & 41,94 \\
2 & 3 & 84,68 & 3,90 & 18,97 \\
3 & 2 & 87,28 & 7,11 & 50,63 \\
3 & 3 & 83,00 & 4,80 & 52,50 \\
3 & 4 & 81,68 & 3,75 & 103,74 \\
4 & 2 & 86,46 & 7,14 & 80,77 \\
4 & 3 & 82,88 & 5,32 & 273,10 \\
4 & 4 & 80,06 & 3,82 & 34,73 \\
4 & 5 & 79,70 & 3,27 & 32,69 \\
5 & 2 & 84,90 & 8,25 & 104,20 \\
5 & 3 & 81,02 & 5,62 & 84,65 \\
5 & 4 & 79,06 & 4,40 & 336,15 \\
5 & 5 & 78,10 & 3,17 & 32,88 \\
5 & 6 & 77,04 & 2,90 & 77,04 \\
\hline
\end{tabular}

Table 6.11: Competition decrease - 5 observations per niche

\begin{tabular}{ccccc}
\hline Nb criteria & Nb niches & Same utility \% & $\begin{array}{c}\text { Avg comp. decrease } \\
\text { (different utilities) } \%\end{array}$ & $\begin{array}{c}\text { max comp. } \\
\text { decrease } \%\end{array}$ \\
\hline 2 & 2 & 88,84 & 5,68 & 33,32 \\
2 & 3 & 87,40 & 2,95 & 12,83 \\
3 & 2 & 90,04 & 5,63 & 45,05 \\
3 & 3 & 87,06 & 4,11 & 105,03 \\
3 & 4 & 84,56 & 3,21 & 123,42 \\
4 & 2 & 90,46 & 5,85 & 44,49 \\
4 & 3 & 86,40 & 4,20 & 65,02 \\
4 & 4 & 84,24 & 2,87 & 30,69 \\
4 & 5 & 83,88 & 2,72 & 43,47 \\
5 & 2 & 88,50 & 7,08 & 224,04 \\
5 & 3 & 85,92 & 4,50 & 127,54 \\
5 & 4 & 83,78 & 2,95 & 88,90 \\
5 & 5 & 82,58 & 2,61 & 70,05 \\
5 & 6 & 83,66 & 2,09 & 30,01
\end{tabular}

Table 6.12: Competition decrease - 7 observations per niche

\subsection{Conclusion and directions for future research}

In this chapter, we have motivated and formalized the notion of bidding niche partitions. To our knowledget this kind of problem is new and this 
chapter only constitutes a first attempt to explore it.

The model proposed still needs to be completed. More precisely, careful attention should be paid to the evaluation of bidding niches partitions. In this work we have considered the difference between maximum intra-set and minimal extra-set competition indices. However, other aggregation operators could be considered and should be tested. Extensions of the present model to characterize single bidding niches (without necessarily belonging to a partition in bidding niches) and outlying bids constitute other research directions.

As pointed out, the choice of using a hierarchical algorithm to find bidding niches may have consequences on the partitions detection. Even if this scheme remain simple, it becomes time consuming when the number of pareto optimal bids increases. Other approaches could be tested and, for instance, integrate the dynamical aspect of the problem. Since the structure of the bidding space do not completely change from one instant to the other (see section 6.4.5), the structure obtained at time $k$ could be exploited to obtain the one at time $k+1$.

If first empirical tests seem to be encouraging, one have to keep in mind that the generation of bidding niche partitions remain an open problem. The good results may have been influenced by the data generation and the model should be tested on more difficult test sets.

Finally, the application of bidding niches partitions as a support system to refine a Butterfly model has just been stressed in the beginning of this chapter. A complete analysis still needs to be done. 


\section{Conclusion}

Multi-attribute auctions constitute an emerging research field. When I engaged in this $\mathrm{PhD}$ thesis, in 2000, only a few contributions were related to it. Nowadays, typing the words Multi-attribute auctions on Google leads to plenty of results including academic and industrial papers, conference talks, web applications, ...

Being at the boundary between multicriteria analysis and auction theories, this field has attracted researchers with different backgrounds and different research interests: from theoretical and experimental economics to web application design.

From the multicriteria perspective, most of the approaches that have been proposed are based on traditional tools such as linear or quasi-linear multi-attribute utility functions, pricing out methods, ... These models induce complete relations when comparing pairs of offers.

In this work, our aim was to investigate the field by means of less restrictive tools, allowing partial relations for the pair-wise comparison of the bids. If this distinctive feature has already been motivated in Decision Aid (in the so-called French school of Multicriteria Decision Aid), it has, to our knowledge, not yet been considered in an auction context.

This idea has led us to, first, analyze the field from an abstract level and, then, to consider a particular model referred to as the butterfly model. Finally, the notion of bidding niches partitions has been presented in order to motivate the use of partial relations. The next section further completes the description of our contribution and presents some of its limitations. Then, some directions for future research are outlined. 


\section{Work summary and critics}

The main contributions of the $\mathrm{PhD}$ thesis are presented in the second part of this work. At first, we have studied the problem from an abstract perspective. Starting with a general preference structure $(P, I, J)$, we have formalized the notion of multicriteria auction and related concepts. This theoretical framework has allowed us to characterize the auction outcomes, to outline potential extensions to Vickrey and Dutch multicriteria auctions and to present restrictions to existing formats. Finally, questions related to ex-post versus integrated bid selection and multicriteria auctions versus RFQ have been addressed. A number of distinctive features and limitations of the model have already been discussed in section 4.3.4. Among them, let us remind the reader about issues concerning information revelation, computational efficiency, the consequences of the auction rules and the cognitive complexity of the bidding process. In addition to these points, a major critic that can be opposed to our approach is that it is nearly exclusively focused on the buyer side: the bidding behavior of the sellers, referred to as the aggressive bidding behavior, may appear too simple in practice (but was necessary to be able to derive theoretical results).

In chapter 5, we have proposed a specific model to conduct multicriteria auctions: the butterfly model. A distinctive feature of this model is that it may be refined during the auction. Starting from a general model (that may be equivalent to a dominance relation), the buyer is allowed to progressively adjust it. A critic that may be opposed to the model is directly related to this aspect. Since the model may evolve during the auction, a seller that enters the auction has no guarantee that the buyer will not favor somebody else by adjusting his model in a biased way ${ }^{14}$. Of course, the model itself restricts the kind of preferences that can be correctly represented. Finally, in order to insure some consistency in the winning bids sequence, the buyer is not allowed to withdraw or modify a refinement that has already been impacted in the model. Therefore, each update restricts future evolutions. Once more, let us remind the reader that a number of distinctive features have already been discussed in section 5.2.4.

Finally, in chapter 6 , we have formalized the notion of partition of the bidding space into niches. Such a context offers a nice application for multicriteria auctions. If the model proposed and the related algorithm have been tested on artificial data sets according to different scenarios, the good results

\footnotetext{
${ }^{14}$ let us note that in other models discount rates are sometimes applied to specific bidders
} 
that have been obtained may be criticized. Indeed, due to the intrinsic difficulty of building test sets characterized by a given optimal allocation, those that have been generated may appear to be too ideal to test the algorithm.

\section{Directions for future research}

According to us, two main contributions will substantially complete the present work. First of all, the development of a web-based interface supporting butterfly auctions will allow us to illustrate and analyze their use. Thanks to this tool, practical questions related to the information revelation and to the cognitive complexity of the bidding process could be addressed through experiments.

Secondly, if the link between the use of partial relations for the pair-wise comparison of offers (in particular the use of the butterfly model) and bidding niches has been outlined, it still requires a thorough analysis. Two aspects have essentially to be considered. On the one hand, the butterfly model could be used in order to maintain at least one winning offer for every niches. Of course, this condition further constraints the evolution of the model. On the other hand, as already stressed, the presence of bidding niches can be exploited to support the buyer for the refinement of the butterfly model (bids belonging to the same niche being compared first). Of course, due to the dynamics of the bidding process, the structure of bidding niches itself is likely to evolve. Managing this aspect adds a further difficulty.

Additionally, a number of specific points that have been outlined in this thesis deserve more attention. Among them, we can cite:

- concerning the theoretical framework: the consideration of more elaborate bidding behaviors for the sellers, their impact on the theoretical results presented in chapter 4 and a thorough analysis of Vickrey and Dutch multicriteria auctions.

- concerning the butterfly model: the testing of the proposed multicriteria bidding support tool, the analysis of the convergence property and the robustness of refinements proposed by the buyer.

- finally, concerning the bidding niches: other stopping rules for the hierarchical algorithm may be considered and the way the artificial data sets on which the tests have been performed can be improved. 
Among the extensions that can be envisaged, multicriteria combinatorial auctions certainly constitute a fruitful research direction. The link between multi-attribute auctions and combinatorial auctions, presented in section 4.3.4, has already put a bridge between these two fields. Additionally, the example of the advertisement problem presented in section 6.1, has illustrated a first interesting link with bidding niches.

To conclude, let us stress that some research questions go beyond the scope of multi-attribute auctions. First of all, the problem of detecting bidding niches partitions can be viewed as a particular example of multicriteria clustering problems and, therefore, offer new challenges. Finally, the auction perspective brings some interest to the potential dynamic aspect of classic MCDA problem such as the choice problematic. 


\section{Chapter 7}

\section{Rand index testing}

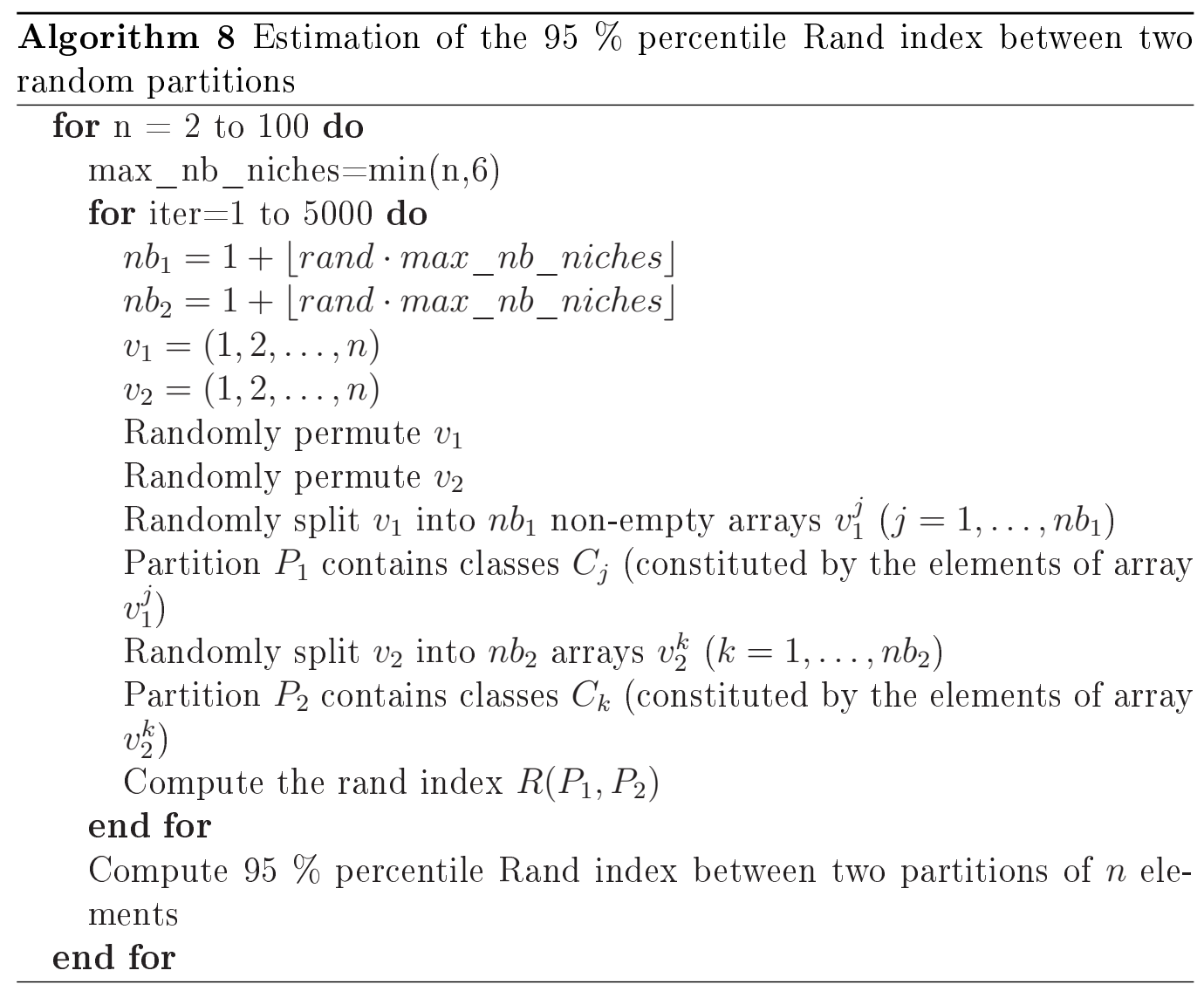




\begin{tabular}{|c|c|c|c|c|c|}
\hline nb obs & $R_{95 \%}$ & nb obs & $R_{95 \%}$ & & \\
\hline 2 & 1 & 36 & 0.64921 & & \\
\hline 3 & 1 & 37 & 0.64715 & nb obs & $R_{95 \%}$ \\
\hline 4 & 1 & 38 & 0.64296 & 70 & 0.62733 \\
\hline 5 & 1 & 39 & 0.64507 & 71 & 0.62938 \\
\hline 6 & 0.93333 & 40 & 0.64231 & 72 & 0.6295 \\
\hline 7 & 0.90476 & 41 & 064024 & 73 & 0.63033 \\
\hline 8 & 082142 & 42 & 8רים 640 & 74 & 0.62736 \\
\hline $\begin{array}{l}0 \\
0\end{array}$ & אבת & 42 & $0.04 \angle 20$ & 75 & 0.62739 \\
\hline 9 & 0.80550 & 43 & 0.63898 & 76 & 0.62702 \\
\hline 10 & 0.77778 & 44 & 0.64165 & 77 & 062697 \\
\hline 11 & 0.76364 & 45 & 0.63939 & 78 & 0.02071 \\
\hline 12 & 0.74242 & 46 & 0.63623 & 10 & 20.027 \\
\hline 13 & 0.73077 & 47 & 0.63969 & 79 & 0.62723 \\
\hline 14 & 0.72527 & 48 & 0.63697 & 8 & 0.6269 \\
\hline 15 & 0.71429 & 49 & 0.6352 & 81 & 0.02932 \\
\hline 16 & 0.70833 & 50 & 0.63918 & 82 & 0.62632 \\
\hline 17 & 0.69853 & 51 & 0.63216 & 8. & 293 \\
\hline 18 & 0.69281 & 52 & 0.63499 & 05 & 0.62823 \\
\hline 19 & 0.68421 & 53 & 0.63353 & 85 & 0.62815 \\
\hline 20 & 0.67895 & 54 & 0.63208 & 86 & 0.62763 \\
\hline 21 & 0.67619 & 55 & 0.63232 & 08 & 0.6231 \\
\hline 22 & 0.67532 & 56 & 0.63442 & $8 \varepsilon^{2}=$ & 0.62591 \\
\hline 23 & 0.67194 & 57 & 0.63221 & مص & 0.02013 \\
\hline 24 & 0.66667 & 58 & 0.63279 & 90 & \\
\hline 25 & 0.66667 & 59 & 0.63296 & 91 & 0.02510 \\
\hline 26 & 0.66462 & 60 & 0.63107 & $\left.9^{\circ}\right)$ & 434 \\
\hline 27 & 0.65812 & 61 & 0.62923 & 93 & 0.62564 \\
\hline 28 & 0.66138 & 62 & 0.63485 & 94 & 0.62617 \\
\hline 29 & 0.65764 & 63 & 0.62724 & & 0.62486 \\
\hline 30 & 0.65057 & 64 & 0.63244 & 96 & 0.62215 \\
\hline 31 & 0.65376 & 65 & 0.62957 & 97 & 0.62736 \\
\hline 32 & 0.64919 & 66 & 0.62844 & 98 & 0.62434 \\
\hline 33 & 0.65152 & 67 & 0.63184 & 99 & 0.62307 \\
\hline 34 & 0.64706 & 68 & 0.6306 & 100 & 0.62354 \\
\hline 35 & 0.64622 & 69 & 0.63171 & & \\
\hline
\end{tabular}

Table 7.1: Estimated 95\% percentile Rand index between two random partitions 


\section{Bibliography}

[1] Aberdeen Group (2002), Making e-sourcing strategic: From Tactical Technology to Core Business Strategy, available on www.marketresearch.com

[2] Amazon auctions, http://auctions.amazon.com

[3] Andersen, J. and Frohlich (2001), FreeMarkets and Online Auctions, Business Strategy Review, 12(2), 59-68

[4] Amsterdam Power Exchange, www.apx.nl

[5] Ariba, www.ariba.com

[6] Asker, J. and Cantillon, E. (2004), Properties of scoring auctions, research paper

[7] AuctionVine, www.auctionvine.com

[8] Bana e Costa, C. (1993), Convictions et Aide à la Décision, Newsletter of the Euro Working Group Multicriteria Decision Aiding, 2

[9] Bellosta, M.J., Brigui, I., Kornman, S. and Vanderpooten, D. (2004), A Multi-criteria Model for Electronic Auctions, ACM Symposium on Applied Computing, SAC 2004, Nicosia, Cyprus, 759-765

[10] Belton, V. and Steward, T. (2002), Multiple Criteria Decision Analysis: An Integrated Approach, Kluwer Academic Publisher, Boston, USA

[11] Bichler, M., Kaukal, M. and Segev A. (1999), Multi-attribute Auctions for Electronic Procurement, Proceedings of the First IBM IAC Workshop on Internet Based Negotiation Technologies, Yorktown Heights, NY, March 18-19 
[12] Bichler, M. (2000), An Experimental Analysis of Multi-Attribute Auctions, Decision Support Systems, 29(3), 249-268

[13] Bichler, M. (2001), The Future of e-Commerce - Multidimensional Market Mechanisms, Cambridge University Press, Cambridge, UK

[14] Bichler, M. and Kalagnanam, J. (2005), Configurable offers and winner determination in multi-attribute auctions, European Journal of Operational Research, 160(2), 380-394

[15] Bloemenveiling Aaslmeer: Facts and Figures 2003, available on www.vba-aalsmeer.nl

[16] Bouyssou, D. and Vincke, P. (2003), Relations binaires et modélisation des préférences, technical report SMG, IS-MG 2003/02

[17] Bouyssou, D. (1993), Décision Multicritère ou Aide Multicritère?, Newsletter of the Euro Working Group Multicriteria Decision Aiding, 3

[18] Branco, F. (1997), The Design of Multidimensional Auctions, Rand Journal of Economics, 28(1), 63-81

[19] Brans, J.P. and Vincke, P. (1985), A preference ranking organization method, Management Science, 31(6), 647-656

[20] Brans, J.P. (1992), Mathématiques de la Gestion: analyse multicritère et optimisation vectorielle, notes de cours, Université Libre de Bruxelles

[21] Brans, J.P. and Mareschal, B. (1992), PROMETHEE V: MCDM Problems with Segmentation Constraints, INFOR, 30(2), 85-96

[22] Brans, J.P. and Mareschal, B. (1994), PROMCALC and GAIA: A new decision support system for Multicriteria Decision Aid, Decision Support Systems, 12, 297-310

[23] Brans, J.P. and Mareschal, B. (2002), Prométhée - GAIA: une méthode d'aide à la décision en présence de critères multiples, Statistiques et Mathématiques Appliquées, Editions de l'université Libre de Bruxelles

[24] Cassady, R. (1967), Auctions and auctioneering, Berkeley: University of California Press

[25] Che, Y.K. (1993), Design competition through multidimensional Auctions, Rand Journal of Economics, 24(4), 668-680 
[26] Chen-Ritzo, C.H., Harrison, T., Kwasnica, A.M. and Thomas, D. (2005), Better, Faster, Cheaper: A experimental analysis of a multiattribute reverse auction mechanism with restricted information feedback, forthcoming in Management Science

[27] Choo, E.U., Schoner, B. and Wedley, W. (1999), Interpretation of criteria weights in multicriteria decision making, Computers and Industrial Engineering, 37 (3), 527-541

[28] Christie's, www.christies.com

[29] Collier, Marsha (2004), Starting an eBay Business for Dummies, 2nd edition, Wiley Publishing

[30] Cripps, M. and Ireland, N. (1994), The design of auctions and tenders with quality thresholds: the symmetrical case, Economic Journal, 104(423), 316-326

[31] De Keyser, W. and Peeters, P. (1994), Argus - A New Multiple Criteria Method Based on the General Idea of Outranking, M.Paruccini (ed.), Applying Multiple Criteria for Decision to Environmental Management, Kluwer Academic Publishers, Dordrecht, 263-278

[32] De Keyser, W. and Peeters, P. (1996), A note on the use of PROMETHEE multicriteria methods, European Journal of Operational Research, 89, 457-461

[33] De Smet, Y. and Gilbart F. (2001), A class definition method for country risk problem, technical report SMG, IS-MG 2001/13

[34] De Smet, Y., Springael, J. and Kunsch, P. (2002), Towards Statistical Multicriteria Decision Modelling: a First Approach, Journal of MultiCriteria Decision Analysis, 11(6), 305-313

[35] De Smet, Y. and Vincke, P. (2002), L'aide à la décision, IT-Scan 7

[36] De Smet, Y. (2003), Multicriteria Auctions: an introduction, proceedings of the 14th Mini-EURO Conference, HCP 2003, Human Centered Processes: Distributed decision making and man-machine cooperation, 277-281

[37] De Smet, Y. (2003), Butterfly auctions: clustering the bidding space, proceedings of the 6th International Conference on Electronic Commerce Research, Dallas, October 2003, 105-115 
[38] De Smet, Y. and Montano Guzman, L. (2004), Towards Multicriteria Clustering: an extension of the k-means algorithm, European Journal of Operational Research, 158(2), 390-398

[39] De Smet, Y. (2005), Multi-criteria Auctions without full comparability of bids, accepted for publication in the European Journal of Operational Research

[40] De Smet, Y. and Lamboray, C. (2005), Clustering the bidding space into niches: a multicriteria hierarchical approach, technical report IS-MG 2005/05 (submitted for publication)

[41] eBay, www.ebay.com

[42] eBay, (2005) Annual Meeting of the Stockholders, www.ebay.com

[43] eBay Live Auctions, www.ebayliveauctions.com

[44] European Energy Exchange, www.eex.com

[45] Federal Communications Commission, www.fcc.gov

[46] Figueira, J, De Smet, Y. and Brans, J.P. (2003), Promethee for MCDA Classification and Sorting problems: Promethee TRI and Promethee CLUSTER, technical report IS-MG 2004/02 (submitted for publication)

[47] Figueira, J., Greco, S. and Ehrgott, M (2004), Multiple Criteria Decision Analysis: State of the Art Surveys, International Series in Operations Research and Management Science, Springer

[48] Fishburn, P.C. (1970), Utility theory for decision-making, Wiley, New York

[49] Friedman, D. and Rust, J. (editors) (1993), The Double Auction Market: Institution, Theories, and Evidences, Santa Fe Institute in the Sciences of Complexity, Proceedings

[50] Grabisch, M. (1995), The application of fuzzy integrals in multicriteria decision making, European Journal of Operational Research, 89, 445456

[51] Green, S. (2003), Privatisation in the former Soviet Bloc: Any lessons for China?, The Royal Institute of International Affairs, briefing note 
[52] Guinness World Records, www.guinnessworldrecords.com

[53] Hites, R., De Smet, Y., Risse, N., Salazar, M and Vincke, P. (2005), About the applicability of MCDA to some robustness problems, accepted for publication in the European Journal of Operational Research

[54] Hou, J. and Rego, C. (2003), A Classification of Online Bidders in a Private Value Auction: Evidence from eBay, proceedings of the Sixth International Conference of Electronic Commerce Research, Dallas, Texas, USA, 139-148

[55] Internet Auction List, www.internetauctionlist.com

[56] Jacquet-Lagrèze, E. and Siskos, J. (1982), Assessing a set of additive utility functions for multicriteria decision making, the UTA method, European Journal of Operational Research, 10(2), 151-164.

[57] Jap, S. (2002), Online Reverse Auctions: Issues, Themes and Prospects for the Future, Journal of the Academy of Marketing Science, 30(4), 506-525

[58] Jelassi, T. and Leenen, S. (2001), Embarking on e-Business at Ducati motorcycles, The $9^{\text {th }}$ European Conference on Information Systems, Beld, Slovenia, June 27-29, 2001

[59] Kagel, H.J. and Roth, A.E. (1995), The Handbook of Experimental Economics, Princeton University Press, Princeton, New Jersey

[60] Kambil, A. and van Heck, E. (2002), Making Markets: How Firms Can Design and Profit from Online Auctions and Exchanges, Harvard Business School Press

[61] Katkar, R. and Lucking-Reiley, D. (2001), Public Versus Secret Reserve Prices in eBay Auctions: Results from a Pokémon Field Experiment, NBER working paper 8183

[62] Keeney, R. and Raiffa, H. (1976), Decisions with multiple objectives; preferences and value tradeoffs, J.Wiley and Sons, New York

[63] Kersten, G.E., Noronha, S.J. and Teich, J. (2000), Are all e-commerce negotiations auctions ?, Proceedings of the COOP'2000: 4th International Conference on the Design of Cooperative Systems, SophiaAntipolis, France, May 2000 
[64] Klemperer, P. (1999), Auction Theory: A Guide to the Literature, Journal of Economic Surveys, 13(3), 227-286

[65] Koppius, O.R. (2002), Information Architecture and Electronic Market Performance, PhD thesis, Erasmus University Rotterdam

[66] Koppius, O.R. and van Heck, E. (2003), Information architecture and electronic market performance in multidimensional auctions, working paper, Erasmus University of Rotterdam

[67] Krishna, V. (2002), Auction Theory, Academic Press, San Diego, California

[68] QED Solutions, www.qed-solutions.be

[69] Luce, R.D. (1956), Semiorders and a theory of utility discrimination, Econometrica, 24, 178-191

[70] Lucking-Reiley, D. (2000), Auctions on the Internet: What's Being Auctioned, and How?, Journal of Industrial Economics, 48(3), 227252

[71] Mareschal, B. and Brans, J.P. (1988), Geometrical representations for $M C D A$, European Journal of Operational Research, 34, 69-77

[72] Marichal, J.L. (1998), Aggregation operators for multicriteria decision aid, $\mathrm{PhD}$ Thesis, Université de Liège

[73] Market Design, www.market-design.com

[74] Maystre, L.Y., Pictet, J. and Simos, J. (1994), Méthodes multicritères ELECTRE: description, conseils pratiques et cas d'application à la gestion environnementale, Presses Polytechniques et Universitaires Romandes, Lausanne

[75] McAffee,R.P. and McMillan, J. (1987), Auctions and Bidding, Journal of Economic Literature, 25, 699-738

[76] McMillan, J. (1995), Why Auction the Spectrum?, Telecommunications Policy, 19, 191-199

[77] Miettinen, K.M. (1999), Nonlinear Multiobjective Optimization, Kluwer Academic Publishers, Boston

[78] Milgrom, P.R. and Weber, R.J. (1982), A Theory of Auctions and Competitive Bidding, Econometrica, 50(5), 1089-1122 
[79] Milgrom, P.R. (2000), An economist vision of the B-to-B marketplace, executive white paper, Perfect

[80] Montano Guzmán, L. (2002), Fuzzy Measures and Integrals in the MCDA Sorting Problematic: Methodology and Application to the Diagnosis of Firms, PhD Thesis, Université Libre de Bruxelles

[81] Myerson, R.B. (1981), Optimal Auction Design, Mathematics of Operations Research, 6, 58-73

[82] Nemery, P. and De Smet, Y. (2005), Multicriteria Ordered Clustering, technical report IS-MG 2003/03 (submitted for publication)

[83] Nisan, N. (2000), Bidding and Allocation in Combinatorial Auctions, Proceedings of the 2nd ACM Conference on Electronic Commerce, 1-12

[84] Noriega, P. (1997) Agent Mediated auctions: The Fishmarket Metaphor, Phd Thesis, Universitat Autonoma de Barcelona.

[85] Office of Government Commerce (2005), eProcurement in action: A Guide to eProcurement for the public sector, available on www.ogc.gov.uk/sdtoolkit/reference/ogc_library/procurement/

[86] Onsale, www.onsale.com

[87] Raiffa, R., Richardson, J. and Metcalfe D. (2002), Negociation Analysis: the science and art of collaborative decision making, The Belknap press of Harvard University Press, 168-169

[88] Rand, W.M. (1971), Objective criteria for the evaluation of clusters methods, Journal of the American Statistical Assosciation, 846-850

[89] Ranjan S., Rangarajan, K.S. (1996), Auction Theory: A Summary with Applications and Evidence from the Treasury Markets, Financial Markets, Institutions and Instruments, V(5),1-36

[90] Resnick, P., Zeckhauser, R., Swanson, J. and Lockwood, K. (2004), The value of reputation on eBay: a controlled experiment, working paper http://www.si.umich.edu/ presnick/papers/postcards

[91] Reverse Auctioneer, www.reverseauctioneer.com

[92] Riley, J.G. and Samuelson, W.F. (1981), Optimal Auctions, American Economic Review, 71(3), 381-392 
[93] Rosenschein, J.S. and Zlotkin, G. (2004), Rules of Encounter: Designing Conventions for Automated Negotiation among Computers, The MIT Press, Cambridge

[94] Rothkopf, M.H., Pekec, A. and Harstad, R.M. (1998), Computationally Manageable Combinatorial Auctions, Management Science, 44(8), 1131-1147

[95] Roy, B. (1968), Classement et choix en présence de points de vue multiples: la méthode ELECTRE, Revue Française d'Informatique et de Recherche Opérationnelle, 8, 57-75

[96] Roy, B. and Bertier, P. (1971), La méthode ELECTRE II: une méthode de classement en présence de critères multiples, SEMA-METRA (Metra International), Direction scientifique, Note de travail 142

[97] Roy, B. (1978), ELECTRE III: un algorithme de classement fondé sur une représentation floue des préférences en présence de critères multilpes, Cahiers du CERO, vol. 20 (1), 3-24

[98] Roy, B. (1990), Decision-aid and decision-making, European Journal of Operational Research, 45, 324-331

[99] Roy, B. (1996), Multicriteria Methodology for Decision Aiding, Kluwer Academic Publishers, Dordrecht

[100] Roy, B. (2000), A French-English Decision Aiding glossary, Newsletter of the Euro Working Group Multicriteria Decision Aiding, 3(1)

[101] Saaty, T. (1980), The Analytic Hierarchy Process, McGraw-Hill, New York

[102] Sandholm, T. (1996), Negotiation among Self-Interested Computationally Limited Agents, Phd Thesis, University of Massachussets, Amherst

[103] Sandhlom, T. (2000), eMediator: A Next Generation Electronic Commerce Server, Proceedings of the International Conference on Autonomous Agents, Barcelona, Spain, 341-348

[104] Sandholm, T. (2000), Distributed Rational Decision Making in Multiagent Systems: A Modern Approach to Distributed Artificial Intelligence, Weiss editor, The MIT Press, Cambridge, Massachussets, 201-258

[105] Sandholm, T. (2000), Approaches to Winner Determination in Combinatorial Auctions, Decision Support Systems, 28, 165-176 
[106] Schärlig, A. (1985), Décider sur plusieurs cirtères: Panorama de l'aide à la décision multicritère, Presses Polytechniques Universitaires Romandes

[107] Sotheby's, http://search.sothebys.com

[108] Smith, C.W. (1989), Auctions: the social construction of value, University of California Press, Berekeley, Los Angeles

[109] Springael, J., De Smet, Y. and Kunsch, P. (2004), A new framework for describing the decision behavior of large groups, internat report IS-MG $2004 / 01$

[110] Stark, R.M. (1974), Unbalanced highway contract tendering, Quaterly Journal of Economics, 69, 99-118

[111] Strecker, S. (2003), Preference revelation in multi-attribute reverse auctions: a laboratory study, Proceedings of the Twenty-Fourth International Conference on Information Systems, 271-282

[112] Strecker, S. (2004), Multiattribute Auctions in Electronic Procurement: Theory and Experiment, PhD Thesis, University of Karlsruhe, Germany

[113] Superior Livestock Auction, www.superiorlivestock.com

[114] Teich, J., Wallenius H. and Wallenius J. (1999), Multiple issue auction and market algorithm for the world wide web, Decision Support Systems, 26, 49-66

[115] Teich, J.E., Wallenius, H., Wallenius, J. and Koppius, O.R. (2004), Emerging multi-issue e-auctions, European Journal of Operational Research, 159(1), 1-16

[116] Teich, J.E., Wallenius, H., Wallenius, J. and Zaitsev A. (2005), A multiattribute e-auction mechanism for procurement: Theoretical foundations, accepted for publication in the European Journal of Operational Research

[117] The Chicago Wine Company, www.tcwc.com

[118] Thiel, S. E. (1988), Multidimensional auctions, Economics Letters, $28(1), 37-40$

[119] uBid, www.ubid.com 
[120] Vansnick, J.C. (1995), L'aide multicritère à la Décision: une Activité Profondément Ancrée Dans Son Temps, Newsletter of the Euro Working Group Multicriteria Decision Aiding, 6

[121] Varian, H.R. (1992), Microeconomic analysis, W.W. Norton \& Co, New York City

[122] Vincke, P. (1989), L'aide multicritère à la décision, Statistiques et Mathématiques Appliquées, Editions de l'université Libre de Bruxelles

[123] Vincke, P. (1992), Multicriteria Decision-Aid, John Wiley and Sons, New York

[124] Vickrey, W. (1961), Counterspeculation, Auctions and Competitive Sealed Tenders, Journal of Finance, 16, 8-37

[125] Visual Decision, www.visualdecision.com

[126] WorldWide Retail Exchange, http://www.worldwideretailexchange.org

[127] Wurman, P.R., Walsh, W. and Wellman, P. (1998), Flexible Double Auctions for Electronic Commerce: Theory and Implementation, Decision Support Systems, 24(1), 17-27

[128] Wurman, P.R. (1999), Market Structure and Multidimensional Auction Design for Computational Economies, PhD thesis, University of Michigan

[129] Yahoo auctions, http://auctions.yahoo.com

[130] Yu, W. (1992), Aide multicritère à la décision dans le cadre de la problématique du tri: concepts, méthodes et applications, $\mathrm{PhD}$ thesis, UER Sciences de l'organisation, Lamsade, Université Paris Dauphine

[131] Yukos, wwww.yukos.com 